\title{
$X$-ray imaging studies of electron cyclotron microwave-heated plasmas in the Tandem Mirror Experiment-Upgrade
}

\author{
B. H. Failor
}

\section{Manuscript date: February 1986}

\section{DISCLAIMER}

\begin{abstract}
This report was prepared as an account of work sponsored by an agency of the United States Government. Neither the United States Government nor any agency thereof, nor any of their employees, makes any warranty, express or implied, or assumes any legal liability or responsibility for the accuracy, completeness, or usefulness of any information, apparatus, product, or process disclosed, or represents that its use would not infringe privately owned rights. Reference herein to any specific commercial product, process, or service by trade name, trademark, manufacturer, or otherwise does not necessarily constitute or imply its endorsement, recommendation, or favoring by the United States Government or any agency thereof. The views and opinions of authors expressed herein do not necessarily state or reflect those of the United States Government or any agency thereof.
\end{abstract}

\section{LAWRENCE LIVERMORE NATIONAL LABORATORY University of California - Livermore, California $\cdot 94550$}



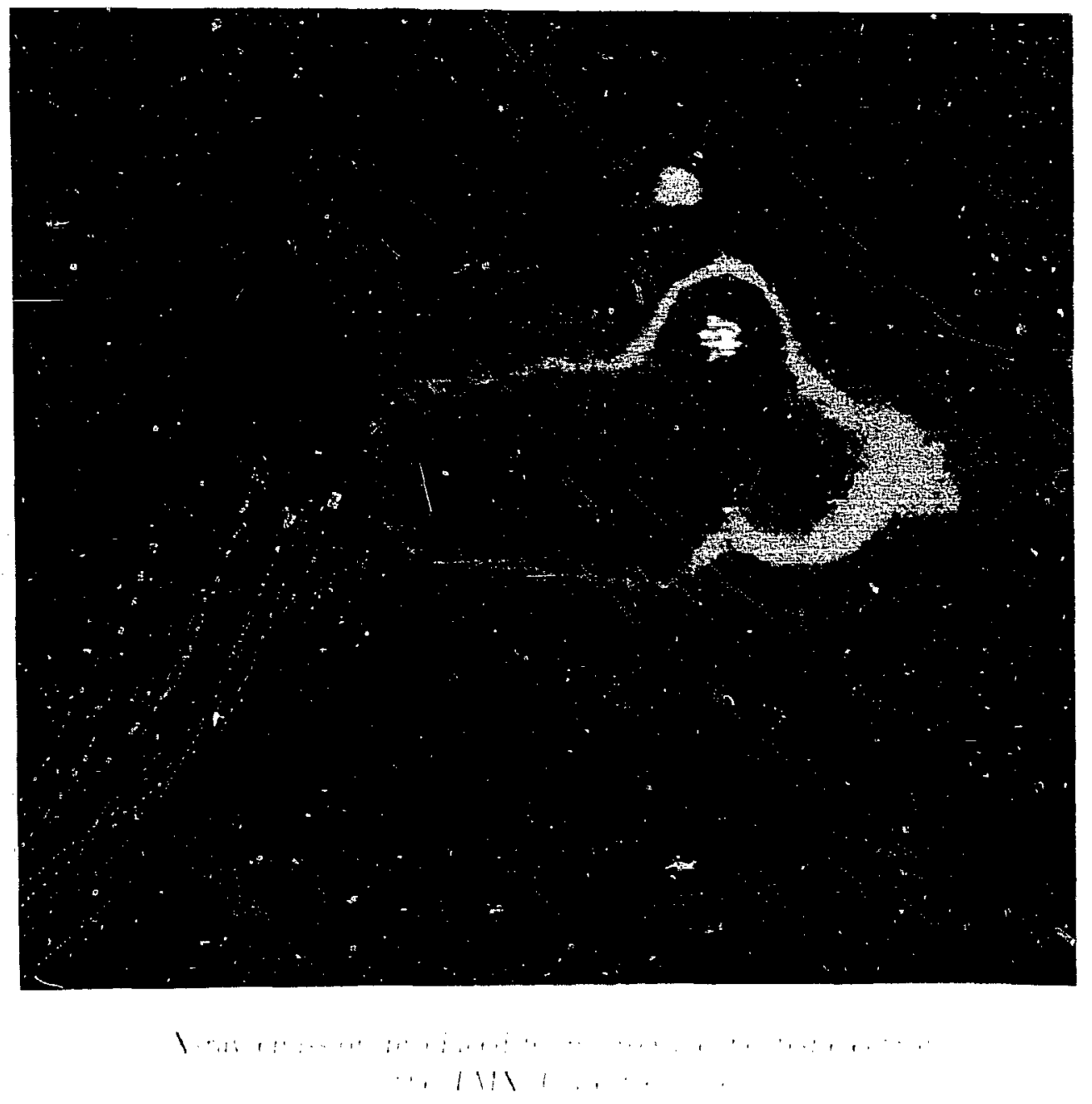


\section{Table of Contents}

Page

List of Figures . . . . . . . . . . . . . . . . . . . . . . . v

Abstract . . . . . . . . . . . . . . . . xi

Acknowledgments . . . . . . . . . . . . . . . . . . . . . . xii

I. Introduction

A. Experimental Overview . . . . . . . . . . . . . 1

B. Summary . . . . . . . . . . . . . . . . . . 7

II. X-Ray Sources

A. Introduction . . . . . . . . . . . . . . . . . 10

B. Plasma Volume Emission . . . . . . . . . . . . . . . . 10

C. Thick Target Emission. . . . . . . . . . . . . . . . . . 19

D. Conclusion . . . . . . . . . . . . . . . . . . . . 22

III. Instrument

A. Introduction . . . . . . . . . . . . . . . . . . . 24

B. Scintillator . . . . . . . . . . . . . . . . 24

C. Intensifiers . . . . . . . . . . . . . . . . . . . . 34

D. Data Recording. . . . . . . . . . . . . . . . . 36

E. Shielding. . . . . . . . . . . . . . . . . . . . . . . . 42

F. Uniformity . . . . . . . . . . . . . . . . . . . . . 45

G. Calibration. . . . . . . . . . . . . . . . . . 45

IV. System Resolution

A. Introduction . . . . . . . . . . . . . . . . . . . . 53

B. Pinhole Size . . . . . . . . . . . . . . . . . . . . . . 53

C. Pinhole Transmission . . . . . . . . . . . . . . 55

D. Scintillator Thickness . . . . . . . . . . . . . . 60

E. Compton Scattering . . . . . . . . . . . . . . . . 70

F. Summary . . . . . . . . . . . . . . . . . . 75

V. Image Analysis

A. Introduction . . . . . . . . . . . . . . . . . . . . . 84

B. Pinhole Collimation . . . . . . . . . . . . . . . 84

C. Plasma Ellipticity . . . . . . . . . . . . . . . . . . . . 88

D. Abel Inversion Technique . . . . . . . . . . . . . . 95

E. Error Analysis . . . . . . . . . . . . . . . . . . . . . 97 
VI. Detected Images
A. Introduction
B. Volume Emission . . . . . . . . . . . . . . . . . . . 102
C. Thick Target Emissions . . . . . . . . . . . . . . . . 102
D. Scintillator Ring . . . . . . . . . . . . . . . . . . . 103
E. Summary . . . . . . . . . . . . . . . . . . . . . . 103

VII. Thick Target Emissions
A. Introduction . . . . . . . . . . . . . . . . . . . . 105
B. Experimental Data . . . . . . . . . . . . . 105
C. Discussion . . . . . . . . . . . . . . . . . . . . . . 119
D. Conclusion . . . . . . . . . . . . . . . . . . . 125

VIII. Volume Emission
A. Introduction
B. Experimental Data . . . . . . . . . . . . 126
C. Discussion . . . . . . . . . . . . . . . . . . . . . . 132
D. Conclusion . . . . . . . . . . . . . . . . . . . . . . 145

IX. Correlations
A. Introduction . . . . . . . . . . . . . . . . . . . . . 148
B. Previous Analysis . . . . . . . . . . . . . . . . . . . 148
C. Present Analysis . . . . . . . . . . . . . . . . . . . 149
D. Conclusion . . . . . . . . . . . . . . . 156

$\mathrm{X}$. Conclusion

A. Introduction . . . . . . . . . . . . 159

B. High Energy X-ray Imaging . . . . . . . . . . . . . . 159

C. Experimental Analysis . . . . . . . . . . . . . . . . . 160

List of References . . . . . . . . . . . . . . . . . . 162 


\section{List of Figures}

Page

I.A.1 Schematic diagram of tandem-mirror axial profiles. . . . . . . . 4

1.A.2 Axial profile of magnetic field in TMX-U, showing microwave heating locations and $\mathrm{X}$-ray camera field of view.

11.B.1 Electron-electron and electron-proton contributions to the continuum bremsstrahlung spectrum. . . . . . . . . . . . . . 14

II.B.2 Electron-electron and electron-proton bremsstrahlung loss rates. . 15

II.B.3 Examples of $\mathrm{x}$-ray spectra detected from the west plug of

TMX-Upgrade. . . . . . . . . . . . . . 18

III.A.1 Schematic of intensified spatially resolving $x$-ray detection system. 25

III.C.1 Comparison of theoretical absorption and ray tracing result for $.5 \mathrm{~mm}$ of CsI-theoretical curves for photoeleciric plus total compton and photoelectric plus absorption compton. . . . . . . . . . . . . . . 28

III.C.2 Comparison of theoretical absorption and ray tracing result for $3 \mathrm{~mm}$ of CsI-theoretical curves for photoelectric plus total cornpton and photoelectric plus absorption compton. . . . . . . . . . . . . . . 29

III.C.3 Difference in absorbed energy fraction between ray trace calculation and photoelectric plus compton absorption divided by ray trace result. $\quad 30$

III.C.4 Absorption efficiencies for .5 and $3 \mathrm{~mm}$ thick CsI scintillators with an 8 mil Al window. . . . . . . . . . . . . . . . . . . 32

III.C.5 Relative power emitted from plasma and deposited in scintillator as a function of the electron temperature $(.5$ and $3 \mathrm{~mm}$ CsI with $8 \mathrm{mil} \mathrm{Al}$ winc'nw).

III.C.6 Diffuse scattering of scintillated light off the side of the crystal would produce an anomalous ring. . . . . . . . . . . . . . 35 
III.D.1 Schematic of camera trigger. . . . . . . . . . . . . . . . . 39

III.D. 2 Density versus exposure curve for 2484 film (green light and HOPE 300 processing). . . . . . . . . . . . . . . . . . . . . . 41

III.E.1 Camera shielding. . . . . . . . . . . . . . . . . . 43

III.F.1 Intensifier nonuniformity. . . . . . . . . . . . . . . . . . 46

III.F.2 Intensifier nonuniformity-axial and radial slices. . . . . . . . 47

III.G.l X-ray film exposure of source used in system calibration. . . . . 49

III.G.2 Comparison of measured (solid) and simulated (dotted) intensities produced by source used to calibrate camera system. . . . . . . . 50

III.G.3 Absolute calibration of x-ray camera. . . . . . . . . . . . . . 51

IV.B.1 Magnification of a pinhole imaging system. . . . . . . . . . . 54

IV.B.2 Resolution of a pinhole imaging system. . . . . . . . . . . 56

IV.C.1 Pinhole design. . . . . . . . . . . . . . . . . . . . . 58

IV.C.2 Effective pinhole cross-sections for $\mathrm{Pb}$ and $\mathrm{U}$. . . . . . . . . . 61

IV.C.3 Degradation of pinhole sharpness at high photon energies. . . . . 62

IV.D.1 Spot size dependence on angle of total internal reflection. . . . . 64

IV.D.2 Spot size dependence on $\mathrm{x}$-ray absorption location. . . . . . . 65

IV.D.3 $I(r)$ in short and long absorption mean free path limits. . . . . . 69

IV.D.4 Variation of spot size with $\mathrm{x}$-ray energy-points marked with $\mathrm{x}(\mathrm{o})$ correspond to .5 (3) $\mathrm{mm}$ thickness of Csl. . . . . . . . . . . . 71

IV.D.5 Variation of spot size with $\mathrm{x}$-ray absorption mean free path. . . . 72 
IV.E.1 Photon interaction processes in CsI. . . . . . . . . . . . . 73

IV.E.2 Difference in averaged intensities with and without Compton scattering in the scintillator-difference divided by peak intensity versus location on fiber optic (3 mm CsI). . . . . . . . . . . . . . . . . . . 76

IV.E.5 Effects of Compton scattering on the shape of the intensity spot produced by different energy $x$ rays in $3 \mathrm{~mm}$ of CsI. . . . . . . . . 77

IV.F.1 Resolution performance of camera for different pinhole diameters and scintillator thicknesses as a function of electron temperature. . . 80

IV.F.2 Comparison of spot sizes produced by a $2.8 \mathrm{~mm}$ diameter pinhole and a $3 \mathrm{~mm}$ thick CsI scintillator of electron temperatures of $5,20,100$, and

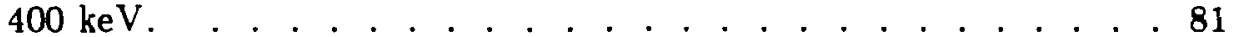

IV.F.3 Comparison of spot sizes produced by a $1.0 \mathrm{~mm}$ diameter pinhole and a $3 \mathrm{~mm}$ thick CsI scintillator of electron temperatures of $5,20,100$, and $400 \mathrm{keV}$.

V.B.1 Collimation effect of pinhole-both the exact result and linear approximation are shown. . . . . . . . . . . . . . . . . 87

V.B.2 Ray trace simulation of the detected image produced by a parabolic $x-$ ray emissivity-no collimation effects. Shown are (clockwise from upper left): a (1) contour, (2) pseudo-3D, (3) radial lineout, and (4) horizontal lineout (each lineout passing through the location of maximum intensity).

V.B.3 Ray trace simulation of the detected image produced by a parabolic $x$ ray emissivity showing collimation effects. Shown are (clockwise from upper left): a (1) contour, (2) pseudo-3D, (3) radial lineout, and (4) horizontal lineout (each lineout passing through the location of maximum intensity). 
V.C.1 Twisted bow tie shape of end plug plasma due to quadrupole fields. 91

V.C.2 Variation in plasma ellipticity in west plug; approximations to the $\mid \mathbf{B}$ surfaces are shown for $z$ values of 530,552 , and $568 \mathrm{~cm}$. . . . . . 92

V.C.3 Transformation to remove ellipticity effects. . . . . . . . . . . 94

V.C.4 Detected signals, and signals corrected for plasma ellipticity. . . . 96

V.E.1 Ratio of total energy deposited to total number of photons deposited as a function of electron temperature $(.5$ and $3 \mathrm{~mm}$ CsI with $8 \mathrm{mil} \mathrm{Al}$ window). . . . . . . . . . . . . . . . . 99

VI.A.1 Sources of x-ray emission in the west end cell of TMX-Upgrade. 101

VII.A.1 Sources of thick target emission with $|\mathbf{B}|$ contours. . . . . . . 106

VII.B.1 Electron guiding center drifts in TMX-Upgrade west end cell. . 110

VII.B.2 Pitch angle required for confinement increase with $x$-axis intercept. 111

VII.B.3 Time variation of thick target emission during heating pulse. . . 113

VII.B.4 Thick target emission during magnet decay. . . . . . . . . . 115

VII.B.5 Comparison of thick target emission for standard (UPHP30) versus SAI magnetic field case. . . . . . . . . . . . . . . . . 116

VII.B.6 Thick target emission for the standard magnetic field case (UPHP30). . . . . . . . . . . . . . . . . . . 117

VII.B.7 Thick ta.rget emission for the SAI magnetic field case. . . . . 118

VII.B.8 Time variation of thick target emission and microwave heating efficiency. . . . . . . . . . . . . . 120

VII.C.1 X-ray detection efficiency and detected flux necessary for shield melting. . . . . . . . . . . . . . . . . . . . 123 
VIII.A.1 Variation of measured stored energy per unit length with the radius of the $\mathrm{x}$-ray emissivity measured by the $\mathrm{x}$-ray pinhole camera. . . 127

VIII.B.1 Short second harmonic heating pulse-shot 9, 20 May 1985. . . 129

VIII.B.2 Long second harmonic heating pulse-shot 19, 20 May 1985. . . 130

VIII.B.3 Radius of $\mathrm{x}$-ray emissivity is larger for longer second harmonic pulse lengths. . . . . . . . . . . . . . . . . . . 131

VIII.B.4 Gaussian and parabolic fits of x-ray intensity data. . . . . . . 133

VIII.B.5 Radial intensity profiles at a seven axial locationsshot 8, 20 May 1985. . . . . . . . . . . . . . . . . 134

VIII.C.1 For fixed peak $\beta$, the second harmonic resonance moves out radially with the hot electron radius. . . . . . . . . . . . . . 136

VIII.C.2 Microwave power and plasma pressure radial profiles in the case of strong absorption. . . . . . . . . . . . . . . . 137

VIII.C.3 Variation of mirror-trapped electron radius obtained from the diamagnetic loop signal normalized to the measured $x$-ray emissivity, adius. . . . . . . . . . . . . . . . . . 138

VIII.C.4 Variation of plasma diamagnetism with bumper limiter location. 139

VIII.C.5 Change in diamagnetic loop signal as bumper limiter is inserted. 140

VIII.C.6 Variation in $g$ with plasma diamagnetism. . . . . . . . . . . 143

VIII.C.7 Experimentally measured variation of $g$ with DML. . . . . . . 144

VIII.C.8 Experimentally measured variation of $g$ with second harmonic power. . . . . . . . . . . . . . . . 146

IX.B.1 Variation of mirror-trapped electron heating rate in TMX-Upgrade end cell as a function of center-cell density and microwave power. . 150 
IX.C.1 Heating efficiency of the second harmonic microwaves depends on stored energy as well as cold electron feed (from center cell). . . . . . 152

IX.C.2 Heating efficiency as a function of center cell density when the stored energy is low. . . . . . . . . . . . . . . . . . . . 153

IX.C.3 Heating efficiency as a function of center cell density when the stored energy is high. . . . . . . . . . . . . . . . 155

IX.D.1 Heating efficiency as a function of center cell density comparing low and high stored energy cases. . . . . . . . . . . . . . . 157 


\section{Abstract}

An $\mathrm{x}$-ray pinhole camera designed to efficiently detect photons with energies between 5 and $250 \mathrm{keV}$ was built to image brei isstrahlung emission from a microwave-heated thot electron plasma. This plasma is formed at one of the thermal barrier locations in the Tandem Mirror Experiment-Upgrade at Lawrence Livermore National Laboratory.

The instrument consists of a lead aferture, an $x$-ray converter in the form of a sodium-activated cesium iodide scintillator, light intensifier electronics, and a recording medium that may either be high speed film or a CCD array. The nominal spatial and temporal resolutions are one part in $\mathbf{4 0}$ and $17 \mathrm{msec}$, respectively. The component requirements for optimum performance were deterr. 'ad both analytically and by computer simulation, and were verified experimentally. The details of these results are presented.

During the last twelve months, the instrument has been used to measure $\mathrm{X}$-ray emission from the TMX-U west end cell. Data acquired with the $\mathrm{x}$-ray camera has allowed us to infer the temporal evolution of the mirror-trapped electron radial profile. Measurements made at different times during the microwave pulse indicate that the radial $\mathrm{x}$-ray emissivity profile broadens as the stored energy in the hated electrons increases. When the stored energy in the plasma is large, the nominal gaussian hot electron radius, as inferred from these measurements is $22 \mathrm{~cm}$. Thick target emission, due to radial losses of energetic electrons, has also been observed, and can be explained by the heating of electrons at radii larger than $18 \mathrm{~cm}$. 


\section{Acknowledgments}

This work would not have been completed without the help and guidance of a number of people.

Dr. Eric H. Silver was my last work supervisor at LLNL. He spent many hours discussing the imaging system with me, and it was through his efforts that the camera design was approved and eventually built. His interest and support was crucial to the success of this study.

Dr. John Clauser and Dr. Peter Poulsen also each in turn acted as my work supervisor during the period of my graduate research on TMX-U. I would like to thank them for their interest in my work and the guidance they provided. I am also grateful to Dr. Robert Horton, of TRW, and Dr. Tom Rognlien, Dr. Reed James, Dr. Barry Stallard, David Ress, and Dr. Tom Simonen, of LLNL, M-Division, for numerous helpful discussions.

Technical and computational support was provided by a number of individuals. Claude Dittmore and the members of L-Division's Technical Photography Department were a vital source of information with respect to the use of film for quantitative experimental measurements. Also of L-division, Hank Finn and Alex Magee were instrumental in getting the SOCK cessing routines, used to analyze the experimental data, transported to and working on the National Magnetic Fusion Energy Computer Center mainframes. Gäry Stone and other individuals working in the Y-Division LEAF Computer Group digitized much of the film data presented in this work. 
Engineering support was provided by Steve Hibbs (mechanical) and Gerry Coutts (electrical), while the technical support needed to field the camera was given by Jim Bowman, Jeff Laird, and Ken Montgomery.

Finally, I must acknowledge the unwavering love, support, and encouragement of my wife, Machell, and my children, Samuel and Tamara. It is to them that this work is dedicated. 
$\mathrm{X}$-ray :maging studies of electron cyclotron microwave-heated plasmas in the Tandem Mirror Experiment-Upgrade

By

Bruce Harlan Failor

B.S. (Brigham Young University, Provo, UT) 1980

M.S. (University of California, Davis) 1982

\section{DISSERTATION}

Submitted in partial satisfaction of the requirements for the degree of

Doctor of Philosophy

in

Engineering-Applied Science

in the

GRADUATE DIVISION

of the

UNIVERSITY OF CALIFORNIA

DAVIS

Approved:

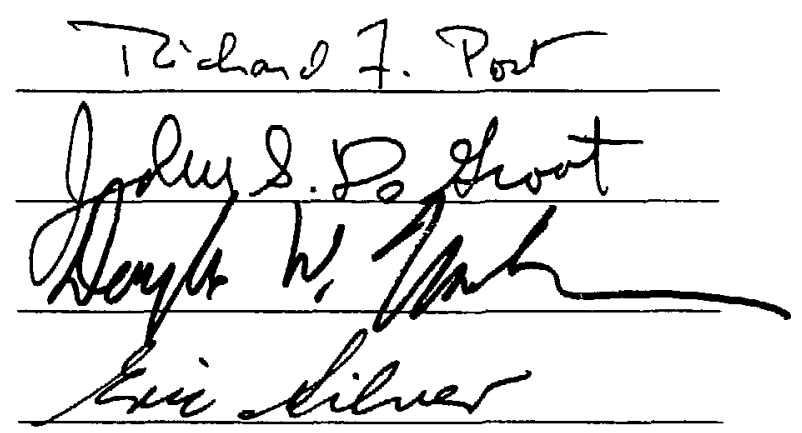

Committee in Charge

Deposited in the University Library

Date Librarian 


\section{Chapter I}

\section{Introduction}

\section{A. Experimental Overview}

A tandem mirror with thermal barriers has been proposed as a candidate for a thermonuclear fusion reactor. ${ }^{1}$ In such a reactor, atomic nuclei would be confined and heated to a sufficient temperature that the repulsive Coulomb potential would be overcome and they would fuse, thus releasing energy that would be used to generate electrical power. The Tandem Mirror Experiment-Upgrade at Lawrence Livermore National Laboratory is the first large scale experiment to test the thermal barrier concept.

In a tandem mirror without thermal barriers, plasma potentials formed in the mirror plugs help confine the plasma produced in the solenoidal center cell. In a single mirror cell, charged particles are confined as long as the ratio of perpendicular to parallel velocity remains sufficiently large. If the electrons and ions are about the same temperature, the electrons experience collisions and scatter in velocity more rapidly than the ions, and for this reason, the electron particle confiuement time is shorter. The fact that electrons are leaving faster than the ions causes a positive potential to form. This potential will increase, attracting electrons and repelling ions, until the electron and ion loss rates have equal. A tandem mirror consists of two single mirror cells (called end cells or end plugs) connected by a relatively long solenoidal cell (called the center cell). The positive potentials that develop in the end cells help to confine ions to the center cell. This concept has been 
verified experimentally on the Tandem Mirror Experiment at LLNL and other experiments. ${ }^{2}$

The potential that develops can be described by the Boltzmann relation

$$
e \phi_{c}=T_{e c} \ln \left(\frac{n_{p}}{n_{c}}\right)
$$

where $e$ is the electron charge, $\phi$ is the ion-confining potential, $T_{e c}$ is the center cell electron temperature, $n_{p}$ is the plug or end cell density, and $n_{c}$ is the center cell density. This relation holds because electrons, produced by gas ionization in the center cell, are free to flow through the plug. Note that for this potential to exist, the density in the end cell must be greater than that of the center cell.

A tandem mirror with thermal barriers differs from a standard tandem in that an ion confining potential can be formed when the plug or end cell density is less than that in the center cell. A schematic of the differences is shown in figure I.A.1. ${ }^{3}$ In the figure $n_{p}$ and $n_{c}$ are the ion densities in the plug and center cell respectively, and $n_{b}^{\kappa}$ is the difference between $n_{p}$ and the density of mirror-trapped electrons at the location of the thermal barrier. By charge conservation $n_{b}^{*}$ is also the passing electron density-the density of electrons which can pass freely through the plug and end cell regions. Thus the depth of the barrier potential is given by the equation (again a Boltzmann relation)

$$
e \phi_{b}=-T_{e c} \ln \left(\frac{n_{c}}{n_{b}^{*}}\right)
$$


where $\phi_{b}$ is the magnitude of the barrier potential and the other variables have been defined above. When the passing electron fraction is sufficiently limited it is possible to drive up an ion-confining potential with the center cell density greater than that in the end cell.

Electron cyclotron microwave heating is used to trap electrons in the end cells. ${ }^{4}$ The microwaves are supplied by $28 \mathrm{GHz}$ gyrotrons at the outer 5 and $10 \mathrm{kGauss}$ points of the magnetic field. See figure I.A.2. The heating at the 5 kGauss point (at twice the electron gyrofrequency or the second harmonic) is employed to create the hot mirror-confined electron population at the thermal barrier. The injection of the microwaves at the $10 \mathrm{kGauss}$ location (where $28 \mathrm{GHz}$ is equal to the electron gyrofrequency or the fundamental electron cyclotron frequency) provides a feed of warm electrons for the 5 kGauss location. It also increases the perpendicular velocity of the electrons at that location so that they will experience a force, $\mathbf{F}_{\|}$, given by

$$
\mathbf{F}_{\|}=-\frac{\mu}{B}[(\mathbf{B} \cdot \nabla) \mathbf{B}]_{\|}
$$

where $\mu$ is the particle magnetic moment and $\mathbf{B}$ is the magnetic field. The subscript || indicates that the force is directed along the magnetic field vector. This force will cause electrons that have been heated to move away from the $10 \mathrm{kGauss}$ point, so that the desired positive potential peak can form. Neutral beam injection pumps cold ions out of the thermal barrier via charge exchange and also creates a sloshing ion distribution. The injection angle, and thus the turning points of the ions in the magnetic mirror, was chosen 


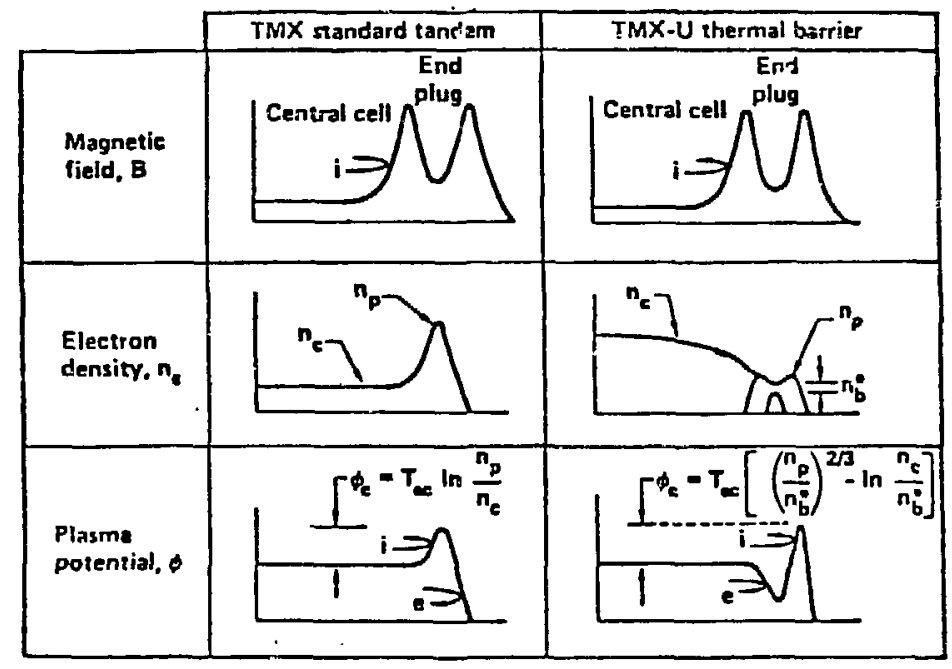

Figure I.A.1. Schematic diagram of tandem-mirror axial profiles. 
so that the ions will have their outer density peak at the location where the $10 \mathrm{kGauss}$ heating is being applied and the ion-confining potential is formed.

The creation of a large mirror-confined electron population is crucial to formation of the thermal barrier. A number of diagnostics have therefore been employed to monitor this population. These include $\mathrm{x}$-ray spectrometer systems, diamagnetic loops, microwave interferometers, and microwave radiometer systems which monitor electron cyclotron emission. The $\mathrm{x}$-ray spectrometers include a $\mathrm{Si}(\mathrm{Li})$ detector, High Purity Germanium detectors, and $\mathrm{NaI}(\mathrm{Tl})$ scintillators, which look at different axial locations in the end cells and also at the end wall. ${ }^{5}$ The spectrometers obtain information about the mirror-confined electron energy distribution. The diamagnetic loops, which are also sensitive to the beam-injected sloshing ions, are spaced at eight different axial locations. ${ }^{6}$ The microwave interferometers measure the plasma dielectric, which depends on the electron density, at a number of axial locations and the electron cyclotron radiometers are located in both end cells, the west system having some ability to scan axially. ${ }^{7}$

Much information about the mirror-trapped electrons has been obtained with these diagnostics. The $\mathrm{x}$-ray spectral measurements indicate that during the high density phase of a shot the electron energy distribution is well characterized by a two temperature, relativistic, anisotropic, Maxwellian, with warm and hot temperatures of $18-25$ and $100-300 \mathrm{keV}$ respectively. ${ }^{8}$ The heating of electrons to hundreds of $\mathrm{keV}$ is undesirable-it is better to have many warm electrons rather than a few hot ones. This is because thermal 


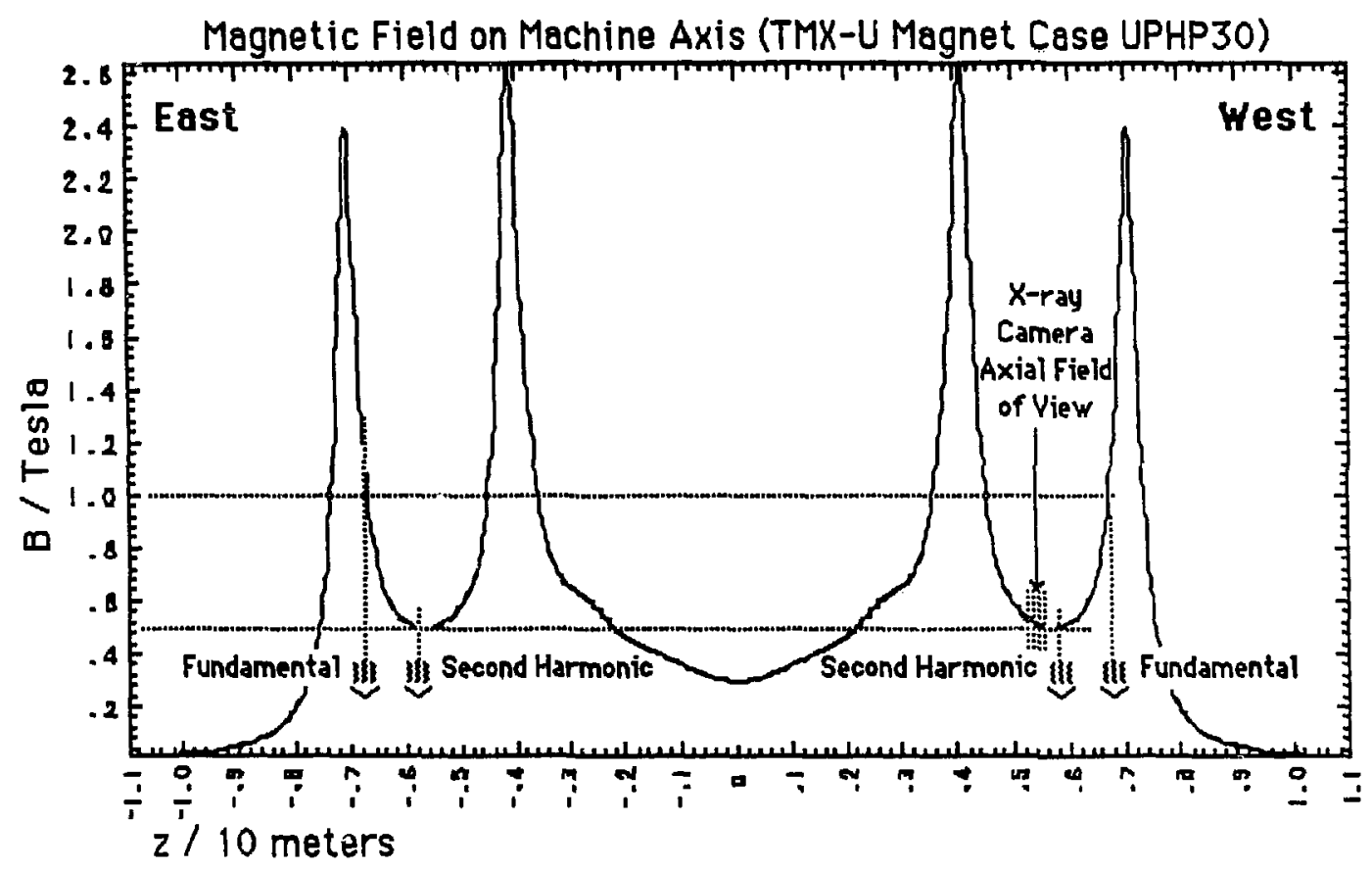

Figure I.A.2. Axial profile of magnetic field in TMX-U, showing microwave heating locations and $x$-ray camera field of view. 
barrier formation depends on the density of mirror-trapped electrons, not their stored energy. The diamagnetic loops measure the stored energy in the mirror-trapped electrons. Stored energies as high as $2.3 \mathrm{~kJ}$ have been achieved and are only limited by the length of the microwave heating pulse. ${ }^{9}$ Perpendicular electron cyclotron emission measurements have been used to obtain information about the axial extent of the mirror-trapped electrons, ${ }^{10}$ but none of the diagnostics mentioned above can easily provide a measure of the hot electron radial profile. For this reason a high energy $\mathrm{x}$-ray imaging system was proposed, conceived, and implemented on TMX-Upgrade.

\section{B. Summary}

This work can naturally be divided into two parts. The first part (chapters II-V) describes how the $\mathrm{x}$-ray imaging system was designed, built, and tested. The second (chapters VI-IX) describes the experimental data that was acquired, and how that data has provided information about the overall performance of the experiment.

In chapter II an overview of the physical processes which can produce

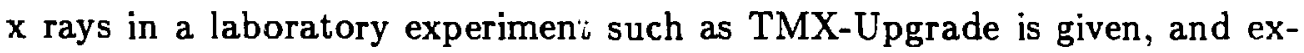
amples of detected X-ray spectra are presented.

Chapter III describes in detail the construction and calibration of the instrument. This includes the scintillator, the light intensifiers, the recording medium, and the imaging aperture and necessary shielding. The instrument 
was calibrated for film operation using a collimated x-ray source of known strength.

A discussion of camera resolution is given in chapter IV. The ideal resolution of the system is degraded due to the fact that the pinhole is not completely opaque to high energy $\mathrm{x}$-rays and also because the scintillator has a finite thickness.

Chapter $\mathrm{V}$ discusses pinhole imaging in general and also how the magnetic topology of the minimum $B$ end cells will affect the acquired image. A prccedure is outlined for removing effects due to pinhole collimation and varying plasma ellipticity.

A description of the images acquired with the instrument is given in chapter VI. Both plasma volume and thick target emission (from solid objects) are observed.

In Chapter VII the thick target emissions are discussed. It is shown that they are the result of heating at large radii, where the electron drifts are poor. The power losses associated with the emission can be significant.

Variations in the volume emission are discussed in chapter VIII. No axial variations are seen, consistent with the fact that the magnetic field gradient is small over the camera field of view. Radial variations have been measured, however, and these are correlated with increases in plasma stored energy. 
The implications of a radial variation in the mirror-trapped electron density profile are examined in chapter IX. Heating efficiency should be degraded as the plasma stored energy increases.

Finally, the conclusions are summarized and suggestions for further work are given in chapter $\mathbf{X}$. 


\section{Chapter II}

\section{X-ray Sources}

\section{A. Introduction}

$\mathrm{X}$ rays are loosely defined as photons with energies greater thail 1 . and less than $1000 \mathrm{keV}$. In an experiment, such as TMX-U, there are a number of ways in which $\mathrm{x}$ rays can be produced, both within the plasma itself and also in the solid objects which surround it. Physical processes which result in $\mathrm{x}$-ray emission from the plasma volume include bremsstrahlung, recombination, and impurity line radiation. When energetic electrons leave the plasma and strike a solid object, "thick target" bremsstrahlung and line radiation can both be produced. In the sections that follow, the dependence of the $\mathrm{x}$ ray emission on different experimental parameters is described for each of the processes mentioned above. In order to use the x-ray emission to diagnose the properties of the microwave-heated electrons, it is important to know how each emission process contributes to the detected $x$-ray flux.

\section{B. Plasma Volume Emission}

As stated above, $\mathrm{x}$ rays are produced in the plasma volume via bremsstrahlung, recombination, and line radiation characteristic of the impurities present. Both bremsstrahlung and recombination produce continuum spectra whose shape depends strongly on the electron temperature. For the electron temperatures, impurity concentrations and ionization states observed during the microwave heating pulse of TMX-Ujpgrade, $x$ rays with energies in the 
range that the $\mathrm{x}$-ray camera is most sensitive, 11 to $183 \mathrm{keV}$, are dominantly produced by bremsstrahlung.

Bremsstrahlung, or "braking radiation." occurs when an electron is accelerated in the electric field of another charged particle. ${ }^{1}$ Bremsstrahlung radiation produced by electron-ion collisions is often used as an electron temperature diagnostic in magneticaliy-confined plasmas. This free-free spectral emissivity, $S_{f f}$, depends on the the electron temperature, $T_{e}(\mathrm{keV})$, the electron density, $n_{e}\left(\mathrm{~cm}^{-3}\right)$, and the density of ions of a given element $(s)$ and charge state $(Z), n_{Z}^{s}\left(\mathrm{~cm}^{-3}\right)$. It is given (in the nonrelativistic limit) by

$$
S_{f f}(E)=\frac{3.05 \times 10^{-15}}{\sqrt{T_{e}}} n_{e} \exp \left(-\frac{E}{T_{e}}\right) \sum_{s} \sum_{Z} Z^{2} n_{Z}^{s} \bar{g}_{f f}\left(E, Z, T_{e}\right)
$$

in units of $\mathrm{keV} /\left(\mathrm{cm}^{3}-\mathrm{sec}-\mathrm{keV}\right) .^{5}$ The quantity $\bar{g}_{f f}\left(E, Z, T_{e}\right)$ is the free-free Gaunt factor, which compensates for the difference between the true energy dependence and the exponential factor in equation II.B.1. It is typically not a bad approximation to set the Gaunt factor to unity, especially in the case of heavier elements. ${ }^{6}$ In the case of hydrogen, for which this approxinuation is the poorest, if $T_{e}$ is $30 \mathrm{keV}, \bar{g}_{f f}$ decreases from .94 at a photon energy of 30 to .62 at $90 \mathrm{keV}$, while the exponential factor goes from .37 to .05 . Because the variation in the Gaunt factor is relatively weak, it has been set to unity for subsequent calculations.

If the energy of an electron is sufficiently large, the ionization state of an impurity is no longer important, and the full nuclear charge should be used to calculate the bremsstrahlung emissivity. This is because the de Broglie 
wavelength of the electron is comparable in magnitude to the electron $\mathrm{K}$-shell radius. $^{7}$ The relationship to be satisfied is

$$
\frac{\hbar}{m v} \leq \frac{.53 \AA}{Z}
$$

In the nunrelativistic limit, the requirement can be written

$$
Z \leq 8.5 \sqrt{W}
$$

where $W$ is the electron energy in keV. Since the camera is sensitive to photon energies above $10 \mathrm{keV}$ (and thus only electrons with at least that much energy), according to the above criterion it is valid to use the nuclear charge for impurities with $Z \leq 27$. Thus the approximation should be good for titanium ( $\mathrm{Ti}$ ), but not for tantalum ( $\mathrm{Ta}$ ). Since the dominant impurities, during the high density phase of a shot, are $\mathrm{O}, \mathrm{N}$, and $\mathrm{C}$, it is therefore acceptable to use the full nuclear charge in equation II.B.2. This approximation collapses the double sum to a single one, with $Z_{s}$ corresponding to the nuclear charge and $n_{Z_{*}}$ to the density of an element (summed over all ionization states). The spectral emissivity is then given by

$$
S_{f f}(E)=\frac{3.05 \times 10^{-15}}{\sqrt{T_{e}}} n_{e} \exp \left(-\frac{E}{T_{e}}\right) \sum_{Z_{\star}} Z_{s}^{2} n_{Z_{\star}}
$$

in units of $\mathrm{keV} /\left(\mathrm{cm}^{3}-\mathrm{sec}-\mathrm{keV}\right)$. The free-free Gaunt factor has been set to one. For a low $\mathrm{Z}$, high electron temperature plasma both electron-ion and electron-electron bremsstrahlung must be considered. ${ }^{2,3}$ As shown in figure II.B.1, the electron-electron contribution (e-e) to the $\mathrm{x}$-ray spectrum is most important at high photon energies, and increases with increased 
electron temperature. ${ }^{4}$ In fact, for a hydrogen plasma, the bremsstrahlung loss rate for electron-electron emission is twice that for electron-ion in the extreme relativistic limit. See figure II.B.2. Thus, if the electron-electron contribution is not included both the density of electrons and their temperature, as calculated from the high energy tail of the $\mathrm{x}$-ray spectrum, will be overestimated.

At photon energies below $200 \mathrm{keV}$ (where the camera is most sensitive), the shape of the spectrum is not strongly affected by the electron-electron contribution, although the magnitude may be. For a hydrogen plasma, when the electron temperature is $32 \mathrm{keV}$ the magnitude is increased by about $15 \%$, while for a $342 \mathrm{keV}$ temperature it is roughly doubled. See figure II.B.1. During neutral beam injection, the presence of impurities increases the electron-ion contribution by about $80 \%$, reducing the relative importance of the electron-electron emission. Thus, although the absolute level of emission may be enhanced by the electron-electron bremsstrahlung contribution, the shape of the spectrum over the energy range that the camera is sensitive is not markedly different than that produced by electron-ion emission.

Another process that produces continuum radiation is recombination or free-bound emission. Because the electron temperature is high compared to the ionization potentials of bound energy states available to the recombining electrons, this process contributes much less to the $\mathrm{x}$-ray spectrum than does bremsstrahlung. If one equates the Gaunt factors for these two processes, the spectral emissivity for a given ion of element $s$ and charge $Z$, considering 

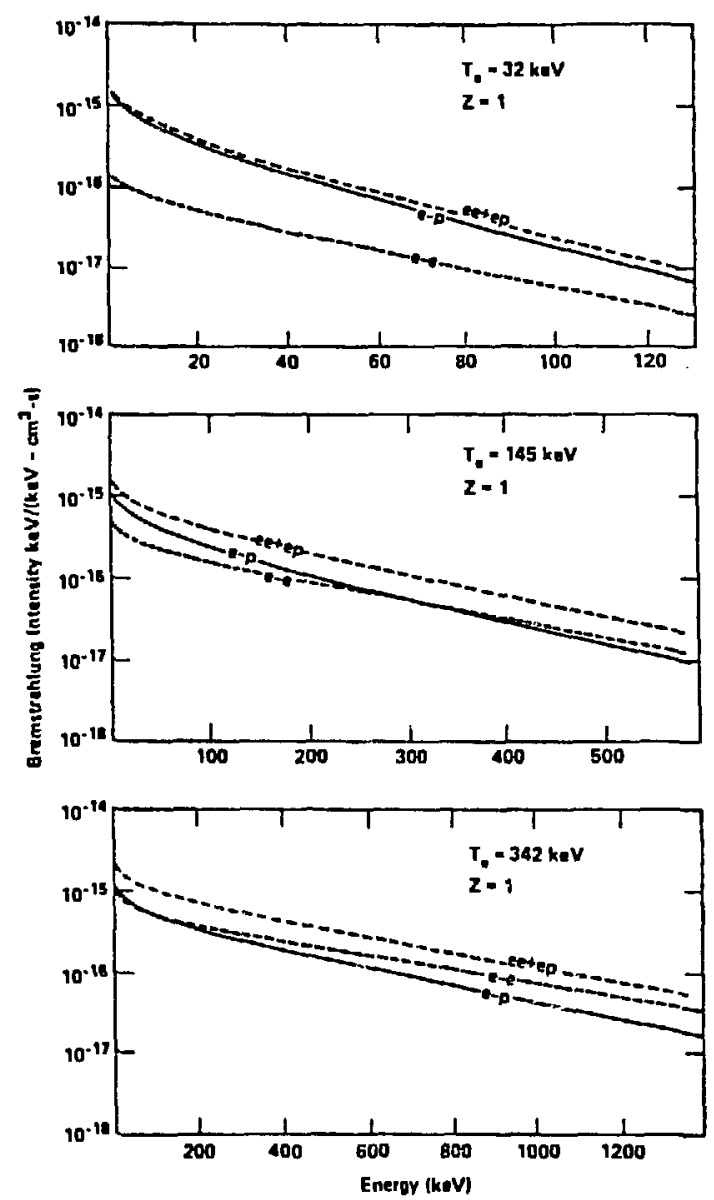

Figure II.B.1. Electron-electron and electron-proton contributions to the continuum bremsstrahlung spectrum. 
Relativistic Maxwellian Plasma

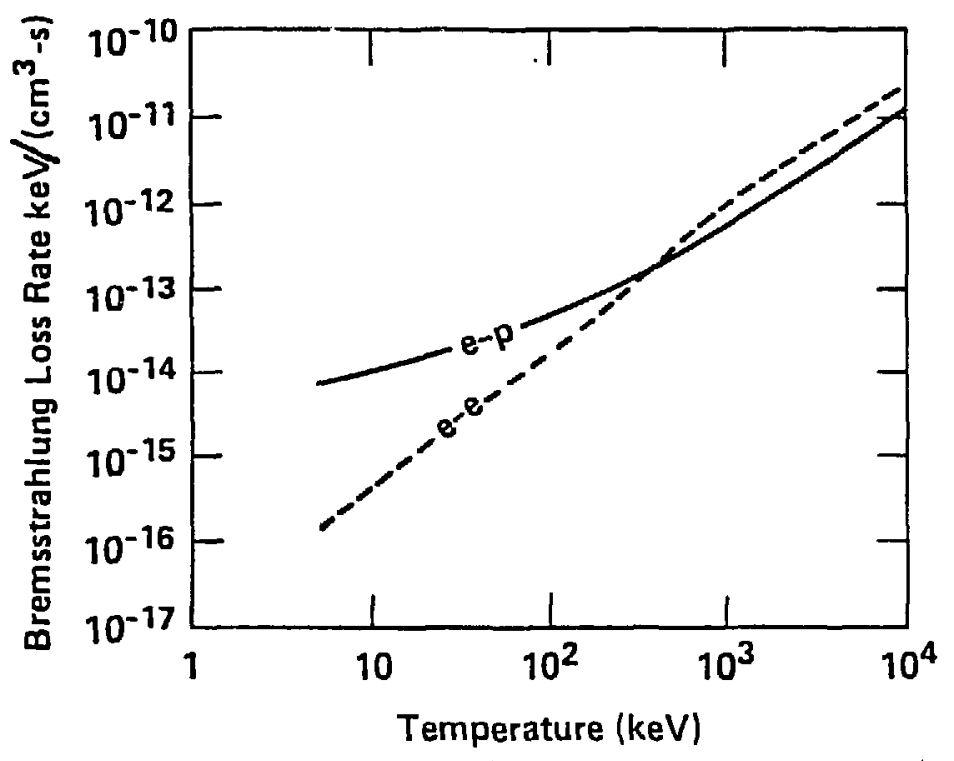

Figure II.B.2. Electron-electron and electron-proton bremsstrahlung loss rates. 
recombination into the shell with principal quantum number $n$, is (in the nonrelativistic limit)

$$
S_{f b}\left(E ; T_{e}, s, Z\right)=S_{f f}\left(E ; T_{e}, Z_{s}\right) \times\left(\frac{n_{Z}^{s}}{n_{Z_{n}}}\right)\left[\frac{2}{n} \frac{\chi_{Z-1}^{n}}{T_{e}} \exp \left(\frac{\chi_{Z-1}^{n}}{T_{e}}\right)\right]
$$

where $\chi_{Z-1}^{n}$ is the ionization potential given by

$$
\chi_{Z-1}^{n}=13.6 \frac{Z^{2}}{n^{2}} \mathrm{eV}
$$

In the limit that any oxygen present was completely stripped, $S_{f b}$ would be over 25 times smaller than $S_{f f}$ if the microwave heated electron temperature was $20 \mathrm{keV}$, while for completely stripped $\mathrm{Ti}$, the two emissivities would be about equal. Since $\mathrm{O}, \mathrm{N}$, and $\mathrm{C}$ were the main impurities present, and the heavy impurities, such as $\mathrm{Ti}, \mathrm{Fe}$, and $\mathrm{Ta}$, are not highly ionized, recombination radiation should not contribute significantly to the $x$-ray continuum emission.

Measurements have been made of the volume continuum emission in the end cells of TMX-Upgrade. Examples of typical spectra are shown in figure II.B.3. The spectra were acquired by two coaligned spectrometer systems which view a chord through the geometric midplane of the west end cell. One system employs an high purity germanium ( $\mathrm{HpGe}$ ) detector and the other a $\mathrm{NaI}$ scintillator coupled to a photomultiplier tube. The measurements indicate that there are two distinct mirror-trapped populations produced by the microwave heating. A warm population which can be characterized by a 
temperature of $18-25 \mathrm{keV}$, and a much hotter component with a temperature of $100-300 \mathrm{keV}$.

High-Z impurities, such as $\mathrm{Ti}, \mathrm{Fe}$, and Ta, will emit characteristic line radiation in the $\mathrm{x}$-ray energy range. The power radiated in a given emission line, $\mathrm{K} \alpha$ for example, by impurity $s$ in charge state $Z$ is given by

$$
P_{L}\left(T_{e}, s, Z\right)=n_{e} n_{Z_{*}}\left(\frac{n_{Z}^{s}}{n_{Z_{*}}}\right)\left\langle\sigma v\left(T_{e}\right)\right\rangle_{s, Z} E_{K \alpha}
$$

in $\mathrm{keV} / \mathrm{cm}^{3}-\mathrm{sec}$, where $E_{K \alpha}$ is the energy of the line and $\left\langle\sigma v\left(T_{e}\right)\right\rangle_{s, Z}$ is the excitation cross section averaged over the electron velocity distribution. Because the spectrometers can not resolve the emissions due to the different charge states of an impurity, a model for the charge state distribution must be used to estimate the fractional abundances of those states. Given a distribution of states, a total excitation cross section can be found by summing the individual cross sections, weighted by the fractional abundances, $\frac{n_{z}^{*}}{n_{z_{x}}}$. Therefore, given $n_{e}$ and $T_{e}$, it is possible to find $n_{Z_{*}}$ for a specific charge state model. ${ }^{8}$

Line emission due to heavy impurities, mainly $\mathrm{Ti}$ and Fe, has been observed in the end cells of TMX-U. ${ }^{9}$ The Ti is gettered onto the machine walls and other structures near the plasma in order to reduce reflux and thus maintain good vacuum conditions. During a shot it can be sputtered off of these surfaces and get into the plasma. In the west end cell, imaging measurements of thick target bremsstrahlung indicate that energetic electrons are striking an Fe shield as well as the tip of a probe made of $\mathrm{W}$ and soldered 

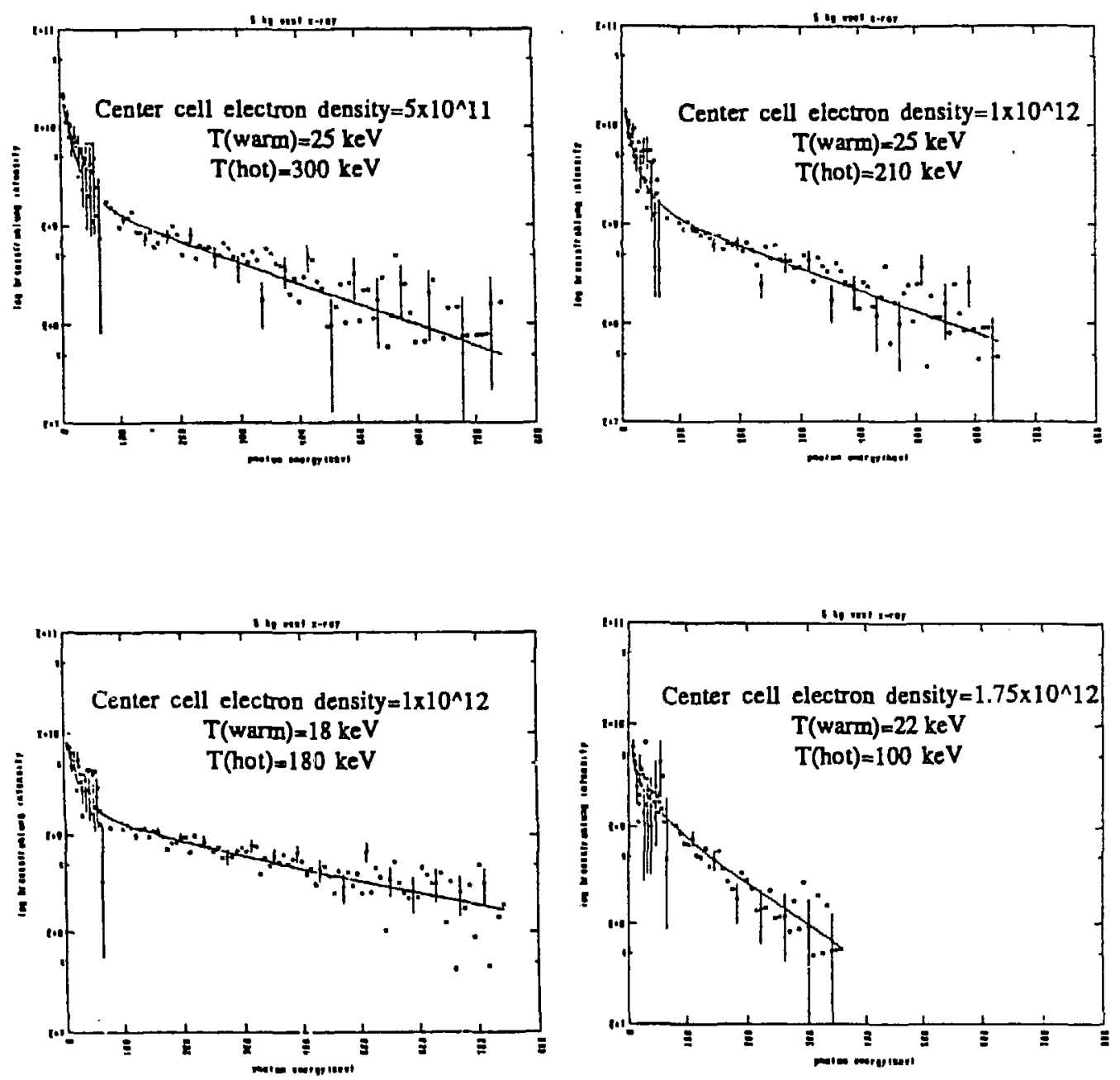

Figure II.B.3 Examples of $x$-ray spectra detected from west plug of TND-Upgrade. 
with Ag. For the shots analyzed in this work, line radiation characteristic of these elements was not typically seen during the high density phase of a shot, however, but rather during the more tenuous long-lived hot electron afterglow. When the plasma density is high, these cold impurity ions are not able to penetrate into the plasma core. Once the cold plasma has decayed away, their mean free path is increased and they are able to come in contact with electrons energetic enough to excite the line radiation.

\section{Thick Target Emission}

Bright $\mathrm{x}$-ray emissions from objects that surround the plasma indicate that some of the energetic electrons are being lost radially. In order to estimate the power associated with these losses the efficiency of thick-target $\mathrm{x}$-ray production must be determined. For electrons with energies below an $\mathrm{MeV}$, the dominant loss mechanisms in a solid absorber are "collisional" (i.e. ionization and excitation) as opposed to "radiative" (bremsstrahlung). Collisional losses are due to multiple collisions with the orbital electrons in the absorber. Radiative losses due to bremsstrahlung occur when a fast electron is deflected in the field of an atomic nucleus. The ratio of the radiative loss rate to the collisional one is approximately given by

$$
\frac{(d W / d x)_{\text {radiatine }}}{(d W / d x)_{\text {collisional }}} \cong \frac{W Z}{700}
$$

where $W(\mathrm{MeV})$ is the electron energy and $Z$ is the atomic number. ${ }^{10}$ This expression indicates that the $\mathrm{x}$-ray production efficiency should increase linearly with the electron energy and the atomic number of the absorber, and experimental measurements, discussed below, confirm this. 
Both the absolute magnitude and the shape of the thick target bremsstrahlung spectrum have been investigated experimentally. ${ }^{11,12}$ When the electron energy is at or below $500 \mathrm{keV}$, the radiation intensity does not vary appreciably with angle, ${ }^{13}$ and the shape of the spectrum for a monoenergetic electron source, with energy $W_{0}$, is given by the simple expression

$$
I_{\text {thick }}(E)=A Z\left(W_{0}-E\right)
$$

where $I_{\text {thick }}$ is the energy radiated isotropically in the photon energy interval, $(E, E+d E)$, and $A$ is a multiplicative constant. This analytic expression was derived by Kramers on the basis of a nonrelativistic, semiclassical calculation which did not take into account electron backscatter in the absorber. ${ }^{14}$ However, experimental results in the low-energy electron region indicated that formula (II.B.2) is satisfactory, at least for order of magnitude estimates. ${ }^{15}$ Different $A$ values have been proposed for general usage. The value $1.4 \times 10^{-3} / \mathrm{MeV}$ provides reasonable agreement with experiment, and has been used in all the subsequent calculations. ${ }^{16,17}$ The bremsstrahlung production efficiency, $\eta\left(Z, W_{0}\right)$ can be calculated by integrating equation (II.B.2) and dividing by the incident electron energy, $W_{0}$;

$$
\eta=\frac{\int_{0}^{W_{0}} d E I_{\text {thick }}(E)}{W_{0}}=7 \times 10^{-7} Z W_{0}
$$

with $W_{0}$ given in keV.

The experimentally measured $x$-ray spectra indicate that the microwaveheated electrons are fairly well described by two Maxwellian energy distributions. Thus an expression equivalent to equation II.B.3 should be found 
for that distribution of electron energies. A general relation for $I_{\text {thick }}$ is

$$
I_{\text {thick }}(E)=A Z \int_{E}^{\infty}(W-E) F(W) d W
$$

where $F$ is the particle flux of electrons with energy $W$ and the other variables have been defined above. Assuming that the $\nabla B$ drift is the mechanism by which the electrons are reaching the solid objects, the drift velocity of a particle will be proportional to its energy, $W .{ }^{18}$ The particle flux is then proportional to $W \exp \left(-W / T_{e}\right)$. Performing the integration, the result is

$$
I_{t h i c k}(E) \propto A Z T_{e}^{2}\left(E+2 T_{e}\right) \exp \left(-\frac{E}{T_{e}}\right) .
$$

The $\mathrm{x}$-ray production efficiency as a function of temperature can be found by integrating $I_{\text {thick }}$ with respect to $E$, and dividing by the total electron energy flux. The result is

$$
\eta\left(Z, T_{e}\right)=2.1 \times 10^{-6} Z T_{e}
$$

The factor of three efficiency increase compared with the monoenergetic case, given in equation II.B.3, can be attributed to the fact that the particle flux is weighted toward higher energy electrons, and the higher energy particles radiate more efficiently.

The experimentally measured spectrum produced by electrons striking and being stopped in an absorber does not typically decrease monotonically with energy, as is the case with the expression given in equation (II.B.4). At low photon energies the self-absorption of the material prevents $\mathrm{x}$-rays from escaping, which causes the radiated spectrum to go to zero at low energy. 
Also, as stated above, fast electrons interact with the orbital electrons of the absorber and can thus excite line radiation characteristic of a material. If the atomic number of the material is 13 (corresponding to aluminum) or higher, some of the radiation will be emitted as $\mathrm{x}$ rays. Thus $\mathrm{x}$-ray tubes with high$\mathrm{Z}$ targets typically produce both a broad continuum and also sharp lines corresponding to the high energy bound-bound transitions of the absorber material.

D. Conclusion

Information about the volume emission bremsstrahlung spectrum obtained with the spectrometers guided the design of the $x$-ray camera. Because $\mathrm{x}$-rays of relatively high energy are produced in TMX-U, the pinho. had to be designed carefully in order to maintain the desired spatial resolution. It was also necessary that the detector itself be sensitive to energetic photons and, because the plasma emissivity was low and the camera had to be located far from the plasma, there had to be sufficient instrumental gain to allow individual $x$-rays to be detected. The way that these criteria were satisfied is described in chapters III through V.

To evaluate the thick target bremsstrahlung, a $125 \mathrm{mil} \mathrm{Pb}$ filter had to be used to reduce the magnitude of the emission from the brightest source. This filter effectively removed any contributions to the detected spectrum due to the characteristic line emission of the target (in this case $\mathrm{Fe}$ ), and also dominated any target self-absorption (the target was about 25 mils thick). 
A further discussion of the thick target emission is given in chapters VI and VII. 


\section{Chapter III}

\section{Instrumentation}

\section{A. Introduction}

The $x$-ray detection system consists of four principle parts: (1) the scintillator, (2) the light intensifiers, (3) the recording medium and (4) the imaging aperture and necessary shielding. Figure III.A.1 shows the relationship of the last three parts of the system. The scintillator absorbs an incident $\mathrm{x}$ ray and converts it to optical photons, which are coupled to the light intensifiers through a fiber optic. The intensifiers amplify the signal so that it can be detected by the recording medium-either film or a CCD array. A description of each of the four components follows.

\section{B. Scintillator}

$\mathrm{CsI}(\mathrm{Na})$ was the scintillator used for all the $\mathrm{x}$-ray imaging measurements. Some of the properties of this scintillator are given in the table below.

\section{Properties of CsI(Na)}

$\begin{array}{ll}\text { Wavelength of Maximum Emission } \lambda_{m} & 420 \mathrm{~nm} \\ \text { Decay Constant } & 0.63 \mu \mathrm{s} \\ \text { Index of Refraction at } \lambda_{m} & 1.84 \\ \text { Density } & 4.51 \mathrm{gm} / \mathrm{cm}^{2} \\ \text { X-ray Scintillation Efficiency } & 11.5 \%\end{array}$

From Scintillation Phosphor Catalog, The Harshaw Chemical Company 

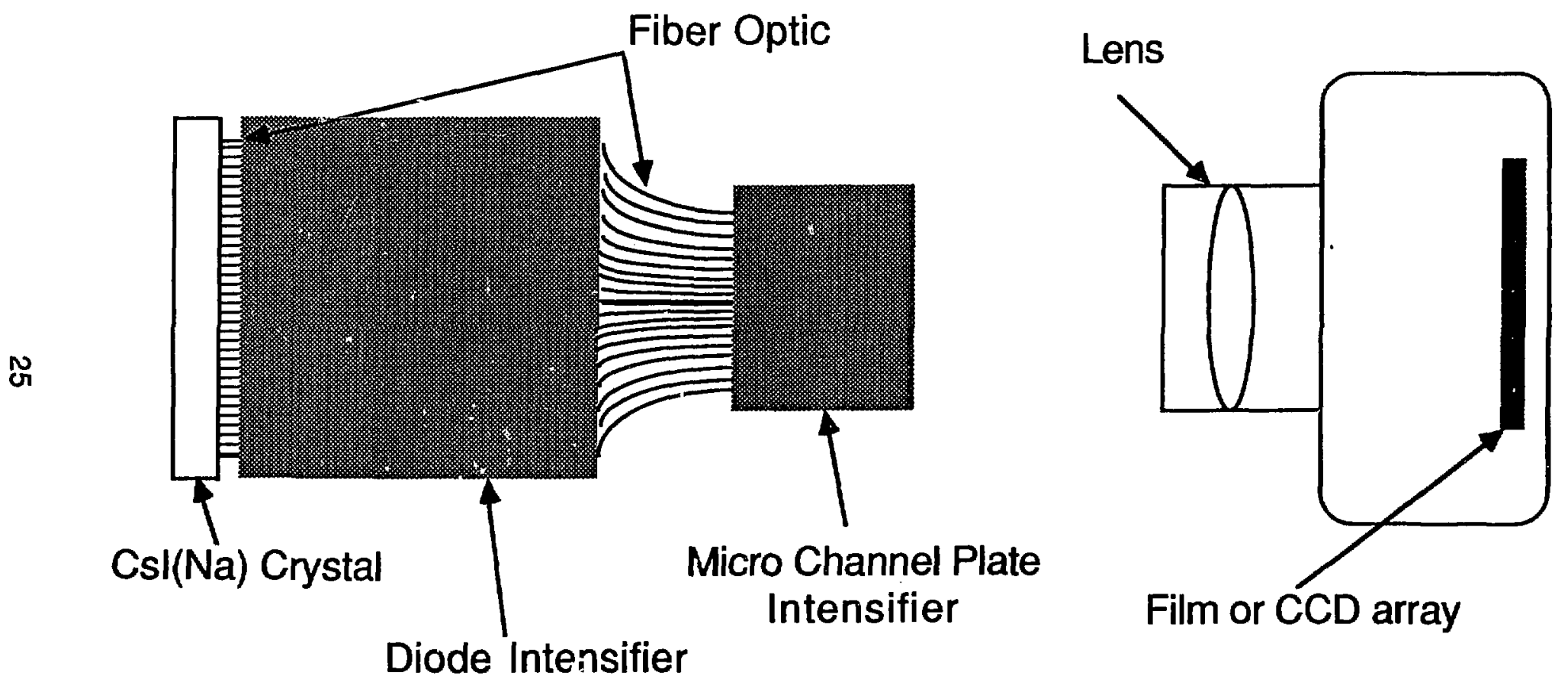

Figure III.A.1. Schematic of intensified spatially resolving $x$-ray detection system. 
For a fiber optic index of refraction of 1.5 , the angle of total internal reflection at the scintillator/fider optic interface is $54.6^{\circ}$. This means that $\left(1-\cos 54.6^{\circ}\right) / 2$, or $21 \%$ of the light emitted at each scintillation makes it into the fiber optic.

The crystal absorption below $1 \mathrm{MeV}$, is determined by the photoelectric and Compton scattering cross-sections. At low energies the photoelectric process is the most important, but above $300 \mathrm{keV}$, Compton scattering becomes the dominant interaction. When Compton scattering is dominant and the thickness of the crystal is equal to or greater than the $\mathrm{x}$-ray mean free path, the amount of absorption is difficult to calculate analytically because multiple Compton events are possible. How the absorption was determined numerically is described below. At $300 \mathrm{keV}$ the $\mathrm{x}$-ray mean free path is about $1 \mathrm{~cm}$. This means that multiple interactions should be more the exception than the rule for the .5 and $3 \mathrm{~mm}$ crystals.

To verify this hypothesis, Monte-Carlo ray tracing runs were done to calculate the absorption. Shown in figures III.C.1 and 2 are the results for the .5 and $3 \mathrm{~mm}$ crystals respectively. On each plot is shown the sum of the photoelectric and total Compton cross-sections (dot), the sum of the photoelectric and Compton absorption cross-sections (dash), and the ray trace results (solid). In both cases the ray trace result lies much nearer the absorption curve predicted using the Compton absorption, as opposed to the total Compton cross-section. ${ }^{1}$ The percent difference between the ray trace results and the Compton absorption predictions are shown in figure III.C.3. 
The solid (dotted) curve corresponds to the .5 (3) mm crystal thickness. As one would expect, the discrepancy increases as Compton srattering becomes more important, but then decreases again as the mean free path for interaction becomes much larger than the crystal thickness. Since the agreement between the ray trace result and the absorption curve predicted by the photoelectric plus Compton absorption cross-sections is good, those cross-sections are used in all absorption calculations.

How Compton scattering within the crystal should affect the camera resolution is discussed in the next chapter. Laboratory measurements, presented in section $\mathrm{G}$ of this chapter, suggest that factors such as the quality of the intensifier electron optics and the sharpness of the lens used to couple the light to the recording medium may influence the resolution more than Compton scattering.

Experimental measurements of the $\mathrm{x}$-ray emission from the TMX-U plasma were made with two different thickness crystals---.5 and $3 \mathrm{~mm}$. The low energy limit of the camera sensitivity is determined by the $\mathrm{x}$-ray transmission of the window on the machine. This window was typically 8 mils of $\mathrm{Al}$, which has a $1 / \mathrm{e}$ transmission point at $11.2 \mathrm{keV}$. The high energy limit is determined by the energy at which $\mathrm{x}$ rays are no longer efficiently absorbed by the scintillator. The high energy $1 / e$ points for the .5 and $3 \mathrm{~mm}$ thicknesses are 95.5 and $183 \mathrm{keV}$ respectively. Absorption curves for the crystals are shown in figure III.C.4 ( $\operatorname{dot}=.5$ and solid $=3 \mathrm{~mm}$ ). To determine how 


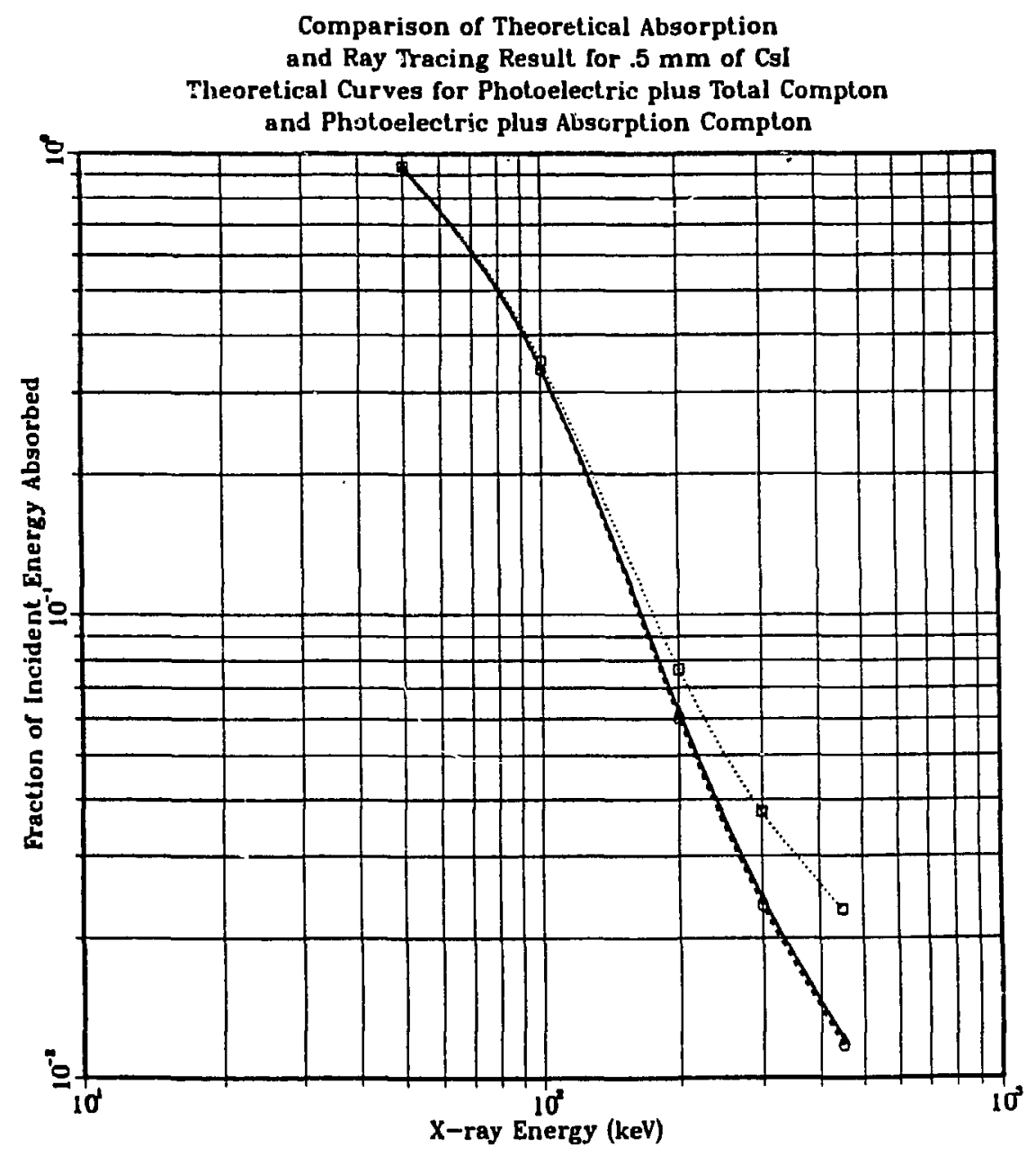

Figure III.C.1. Curves shown include ray trace result (solid), Compton absorption plus photoelectric (dashed), and total Compton plus photoelectric (dotted). 


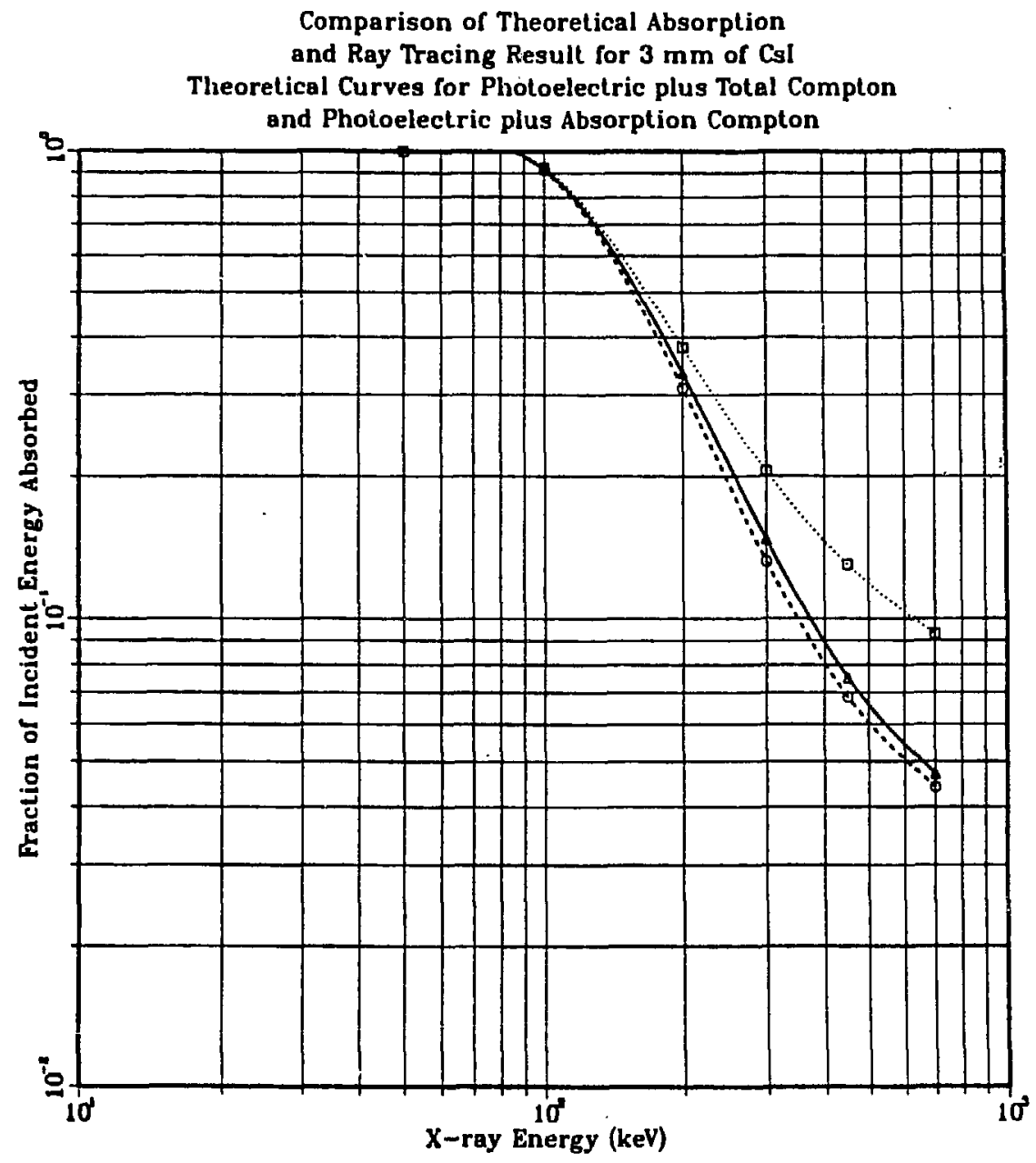

Figure III.C.2. Curves shown include ray trace result (solid), Compton absorption plus photoelectric (dashed), and total Compton plus photoelectric (dotted). 


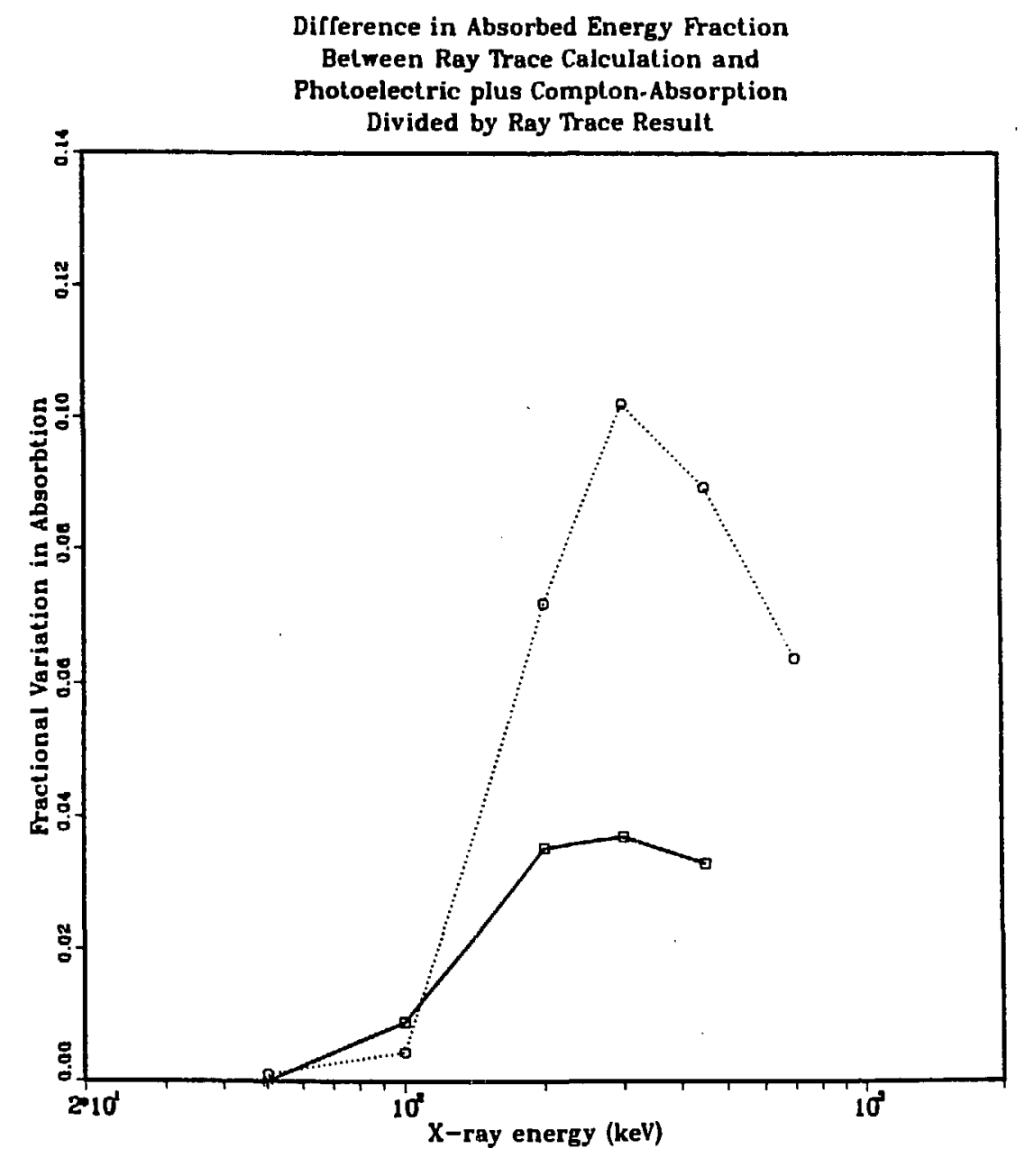

Figure III.C.3. Curves shown include results for .5 (solid) and $3 . \mathrm{mm}$ (dotted) thicknesses. 
the energy deposition in the crystal varies with electron temperature, $T_{e}$, the absorption curves were multiplied by the function, $P(E)$, where

$$
P(E) \propto \frac{\exp \left(-\frac{E}{T_{e}}\right)}{\sqrt{T_{e}}}
$$

and then integrated with respect to $E$, the x-ray energy. The results of the integrations are shown in figure III.C.5. The peak signal for the $.5(3) \mathrm{mm}$ crystal is at a $T_{e}$ of about $110(200) \mathrm{keV}$, but this maximum is a broad one-the signal varying little above $30(50) \mathrm{keV}$.

When analyzing the image data, one needs to compensate for a spurious ring at the crystal edge. This ring is the result of light trapped in the crystal by total internal reflection being reflected diffusely off the crystal side. An analytic calculation of this intensity, $I_{\text {ring }}$, as a function of the distance from the axis of the crystal, $r$, is given by

$I_{\text {ring }}(r) \propto \begin{cases}\frac{\sin \phi_{\max } G(\theta, \alpha)}{\sqrt{r_{0}^{2}+r^{2}}}-\frac{G(\theta, \beta)}{\sqrt{r_{0}^{2}+r^{2}+b_{\max }^{2}}}, & \text { if } r \geq r_{0}-b \tan \phi_{\max } \\ 0, & \text { otherwise. }\end{cases}$ where

$$
\begin{aligned}
& \theta=\arccos \left(\frac{r_{0}^{2}+r^{2}-b^{2} \tan ^{2} \phi_{\max }}{2 r_{0} r}\right) \\
& \alpha=\frac{2 r_{0} r}{r_{0}^{2}+r^{2}} \\
& \beta=\frac{2 r_{0} r}{r_{0}^{2}+r^{2}+b^{2}}
\end{aligned}
$$

and

$$
G(x, y) \equiv \frac{2}{\sqrt{1+y}} F\left(\arcsin \sqrt{\frac{(1+y)(1-\cos x)}{2(1-y \cos x)}}, \sqrt{\frac{-y}{1+y}}\right)
$$




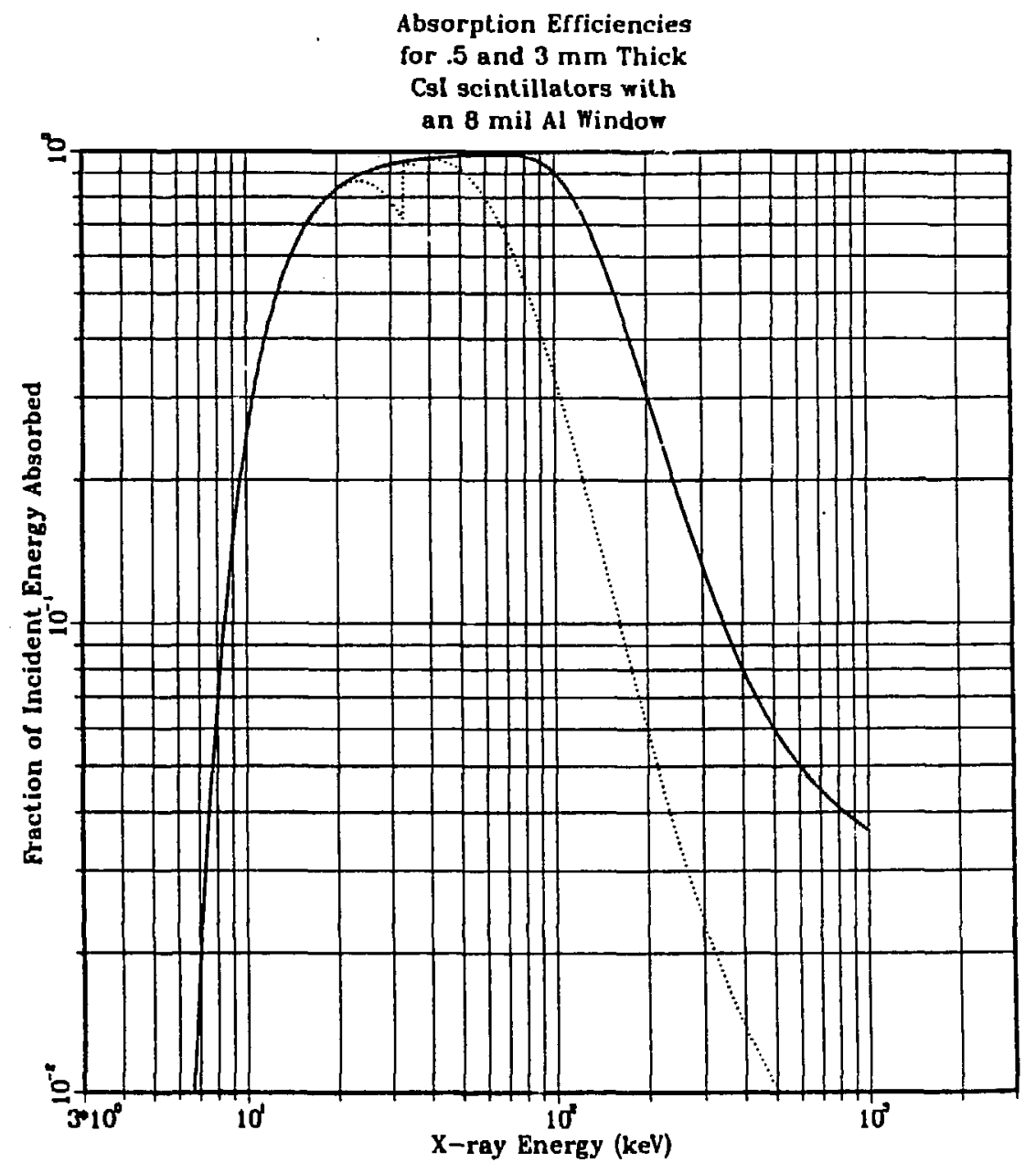

Figure III.C.4. Thicknesses shown include .5 (dotted) and $3 \mathrm{~mm}$ (solid). 


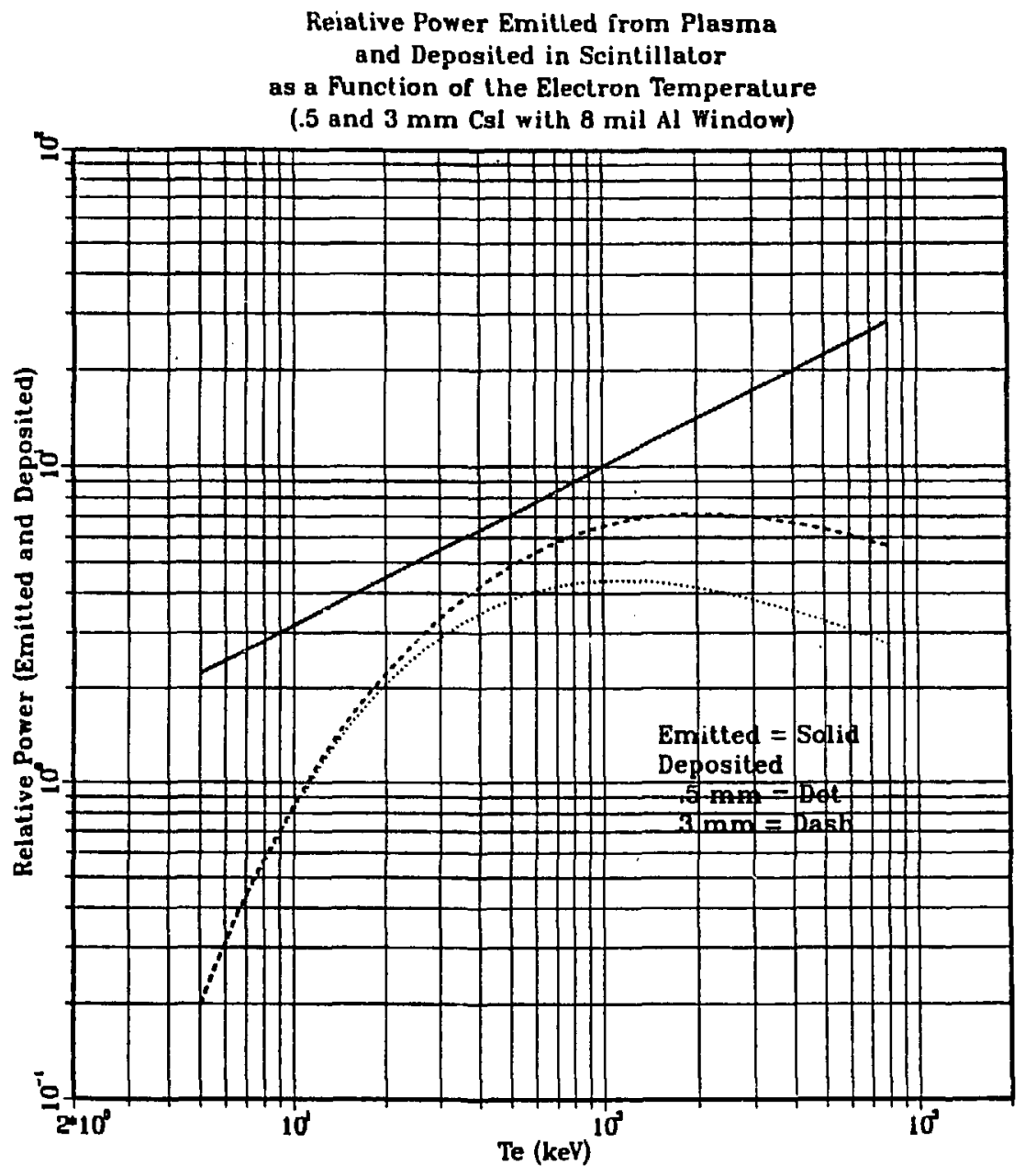

Figure III.C.5. 
$F$ is the first elliptic integral, while $b$ and $r_{0}$ are the height and radius of the crystal respectively, and $\phi_{\max }$ is the angle of total internal reflection at the crystal fiber optic interface. In figure III.C. 6 is shown a comparison between the analytic expression for $I_{\text {ring }}$ and the result of a ray trace run normalized to the same value. A scintillator with a $20 \mathrm{~mm}$ radius and $3 \mathrm{~mm}$ thickness was modeled. The ray trace and analytic results are in satisfactory agreement (within $5 \%$ ). The ring is seen experimentally (see Chapter VI), but it is difficult to quantitatively compare the detected signal with that predicted theoretically, mainly because the intensifier gain is very nonuniform at the edge (see section $F$ of this chapter). It does appear that the ring extends further into the center of the crystal than expected, which again may be the result of less than perfect intensifier electron optics. This effect is discussed further in section $G$ of this chapter.

\section{Intensifiers}

Intensifiers are needed to amplify the scintillated light so that it can be recorded on film or in some other manner. As shown in figure III.A.1 of this chapter, two are used-a $40 \mathrm{~mm}$ diode intensifier followed by a $25 \mathrm{~mm}$ microchannel plate intensifier. A 2:1 fiber optic reducer couples the two. Two intensifiers were used' so that there would be sufficient gain to allow lens coupling of the light to the film.

Both intensifiers operate in approximately the same manner, although the microchannel plate (MCP) intensifier has electron multiplication, while the diode does not. When an $\mathrm{x}$ ray is absorbed in the CsI(Na) scintillator, a 


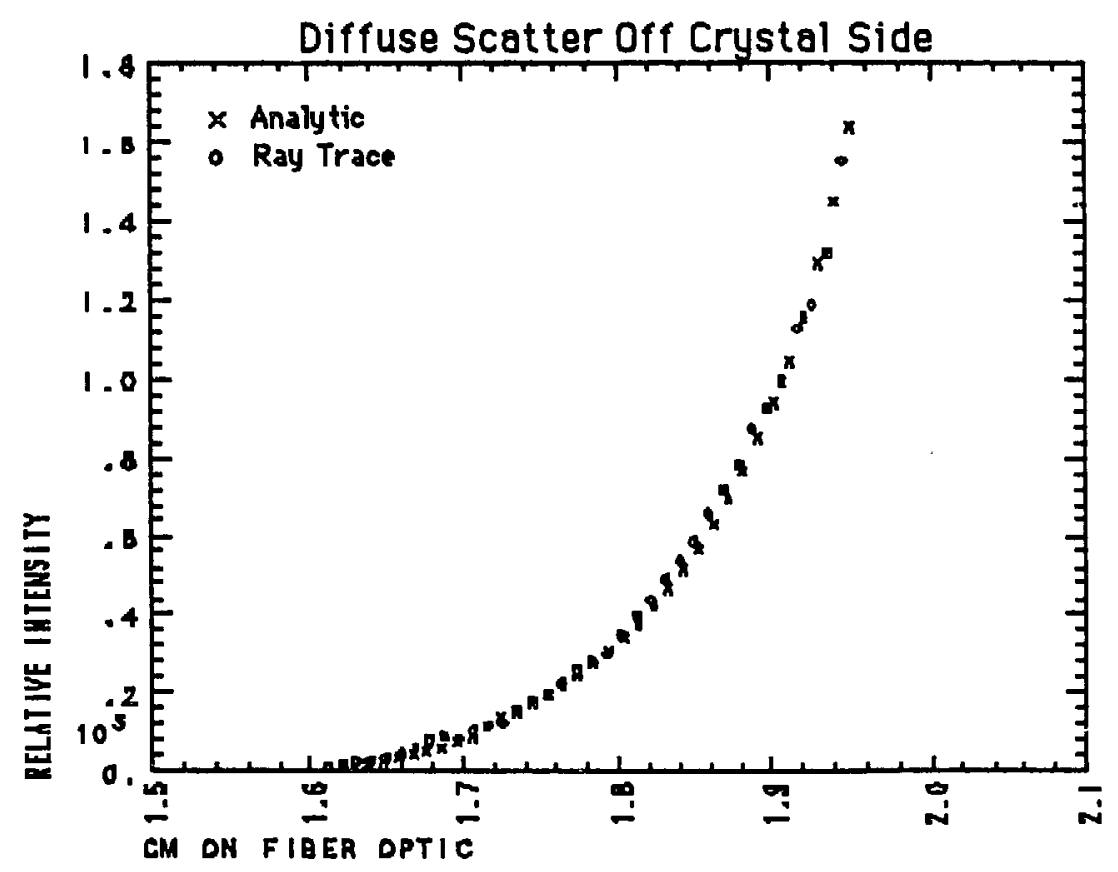

Figure III.C.6. Diffuse scattering of scintillated light off the side of the crystal would produce an anomalous ring. 
large number of optical photons are produceu-the number proportional to the energy of the $x$ ray. These optical photons pass from the scintillator into a fiber optic faceplate. The scintillator faceplate is coupled with optical grease to a similar fiber optic faceplate on the front of the diode intensifier, and by travelling through this interface the scintillated photons eventually reach the photocathode of the diode intensifier. (There is an identical interface between the output of the diode intensifer and the 2:1 reducer, and between the reducer and the front of the MCP intensifier.) At the photocathode, the photons are absorbed and produce photoelectrons. These photoelectrons are accelerated thrcugh a potential and then focused onto the phosphor of the diode intensifier, where optical light is again produced. This light is then fiber-optically coupled, through a $2: 1$ reducer, onto the photocathode of the MCP intensifier. Photoelectrons are again produced, which are then both multiplied and accelerated as they travel through a microchannel plate section. When the electrons emerge from the end of that section they are accelerated into a phosphor, producing the green light that is recorded on film or with a CCD array. The gain of the intensifier chain depends on the quantum efficiency of the photocathode. the electron energy gain (and multiplication in the case of the MCP), and the efficiency of the phosphor in converting the electron energy to light. There are also losses when the light is coupled from one intensifier into the other through the fiber optic reducer. All of these factors were not measured individually, but the total gain of the system was determined using an $\mathrm{x}$-ray source calibrated with a $\mathrm{NaI}$ spectrometer. The $\mathrm{x}$-ray camera calibration is described in section $\mathrm{G}$ 
of this chapter. The measured gain compares well with the value one would estimate from the individual component gains.

\section{Data Recording}

The image formed on the back of the microchannel plate intensifier was recorded two ways: (1) with a standard film camera and (2) with a charge coupled device $(C C D)$ video camera.

The film camera used was a Nikon FE2 with a motor drive. Use of the motor drive allowed the film to be advanced remotely, and made the acquisition of up to three exposures per shot possible (separated by a minimum of $370 \mathrm{msec}$; some hot electrons remained confined until the magnet currents decayed away at approximately 1 second). A schematic of the trigger system is shown in figure III.D.1. The camera was triggered with a gating pulse and a relay chip, the relay located with the camera. Jitter in the trigger was about one millisecond, which was acceptable since the typical exposure time was $\frac{1}{60}$ second or $17 \mathrm{msec}$. The camera shutter consists of two Ti curtains located at the film plane, ${ }^{2}$ and the sweep time of a curtain (from top to bottom of the film) was measured to be less than 2 msec. The operation of the camera was monitored through the two electrical contacts on the hot shoe. When the camera fired and the film advanced successfully, a voltage would appear across the contacts for a short time and then decay away. If the camera did not trigger, no voltage would appear. If it triggered but the film failed to advance, the voltage would appear but would not decay away. So, if other mishaps could be avoided (like the film coming loose from the 
take-up spool and therefore not advancing), the functionality of the camera could be externally monitored.

To maximize the amount of light that reaches the film, it is best to use the fastest available lens and locate it as close as possible to the back of the MCP intensifier. Also, decreasing the size of the image on the film will increase the incident intensity. This is important for operation right at the threshold of the recording medium. A $55 \mathrm{~mm}$ Nikon f1.4 lens was typically used for the film measurements, and an extension ring allowed the camera to be moved closer to the back of the intensifier. A set of close-up lenses proved useful because the camera could be placed even closer than possible with the extension ring alone. With this variety of lens combinations a focus could be obtained for an arbitrary source to image plane distance. For typical operation, the magnification from the back of the MCP to the film plane was 1:.729, and the distance from the MCP to the lens was $10.5 \mathrm{~cm}$. Given this source/lens separation and the size of the lens aperture, approximately $6 \%$ the light is collected.

A high speed panchromatic film (Kodak 2484) was used to record the images. 2484. The film was processed either for 4 minutes at room temperature $\left(20^{\circ} \mathrm{C}\right)$ in Rapid X-Ray Developer (RXR) or with a HOPE film processor at a speed of 300 . Since the phosphor on the back of the MCP intensifier emitted green light, a P-20 phosphor continuous wedge was processed at the same time as the data. The wedge provides a density versus 


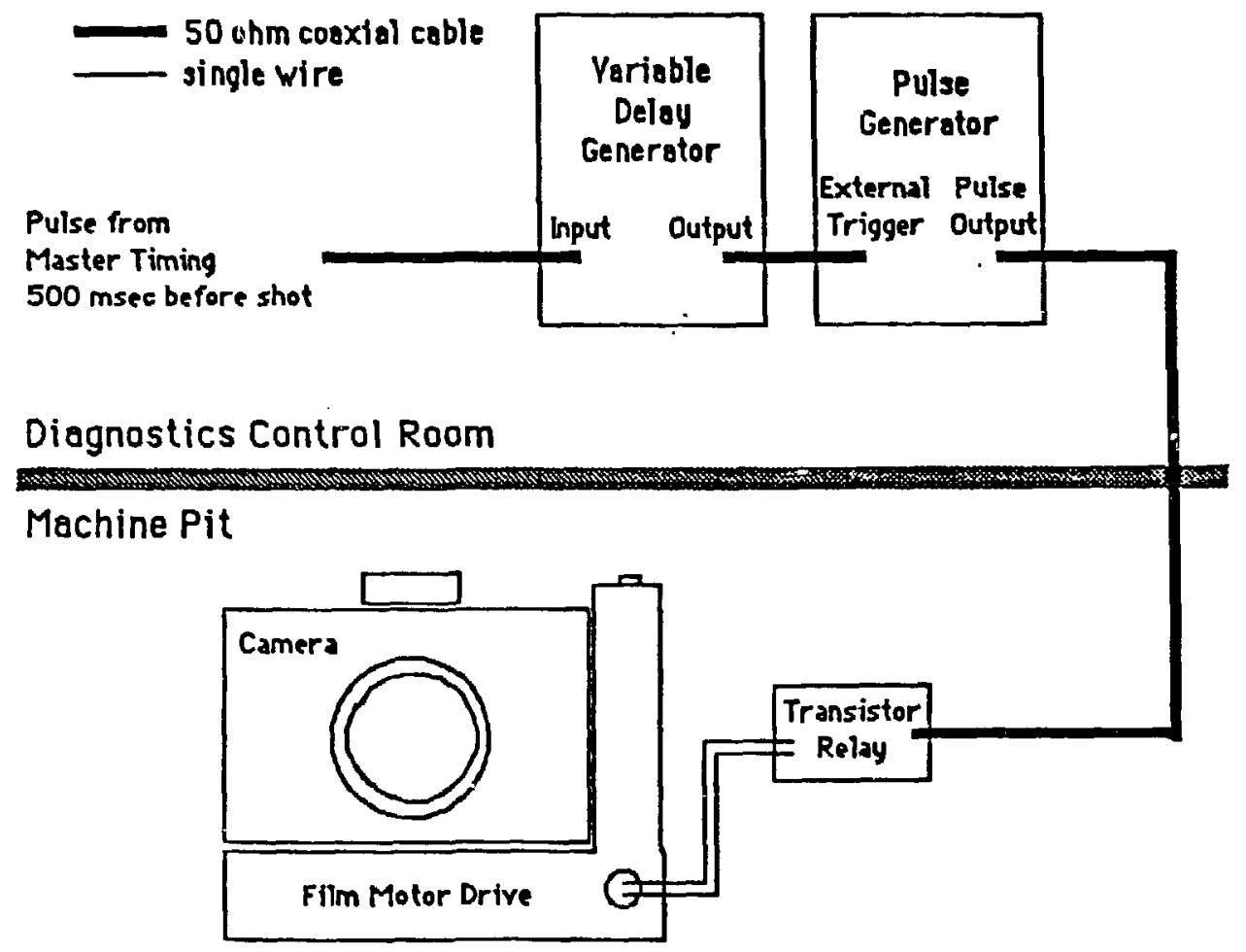

Trigger Timing

Moster Timing Pulse

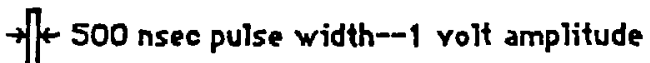

Output of Deloy Generstor

S Selected Delay _

Output of Pulse Generator

$+2100 \mathrm{msec}$

5 volts

Figure III.D.1. Schematic of camera trigger. 
exposure curve for the film. An example of such a wedge, for HOPE 300 processing, is shown in figure III.D.2. The curve indicates an intensity threshold of about $10^{-2} \mathrm{erg} / \mathrm{cm}^{2}$ for green light when $2484 \mathrm{film}$ is processed in this manner. One of the advantages of using film is the broad dynamic range, in this case roughly 3 orders of magnitude (the CCD video camera behaves linearly over approximately two orders of magnitude). ${ }^{3}$

After processing, the data and associated wedge are digitized with a microdensitometer. Typically a 50 micron square aperture was used for this. Once digitized the data was written onto magnetic tape. The data could then be read off the tape onto the computer system used to analyze the data.

By using a CCD camera, multiple images $(\sim 50)$ could be obtained during a shot. Each location in the CCD array was read out and cleared once every $16.7 \mathrm{msec}$ (consistent with a rate of $60 \mathrm{Hertz}$ ), which is the time required to scan the entire image. This means that the first and last pixels scanned are separated by this scan time, even though they are included in the same frame. The corresponding time separation for the film camera was less than 2 msec. Also, the CCD camera clock was not synchronized with the experimental timing system, which means that the beginning of a shot did not correspond to the beginning of a scan. For this reason error bars of $\pm 8 \mathrm{msec}$ in time should be put on each image.

The area of the CCD array was about one quarter that of a standard $35 \mathrm{~mm}$ film frame. For this reason a different lens was used-a $28 \mathrm{~mm}$ 


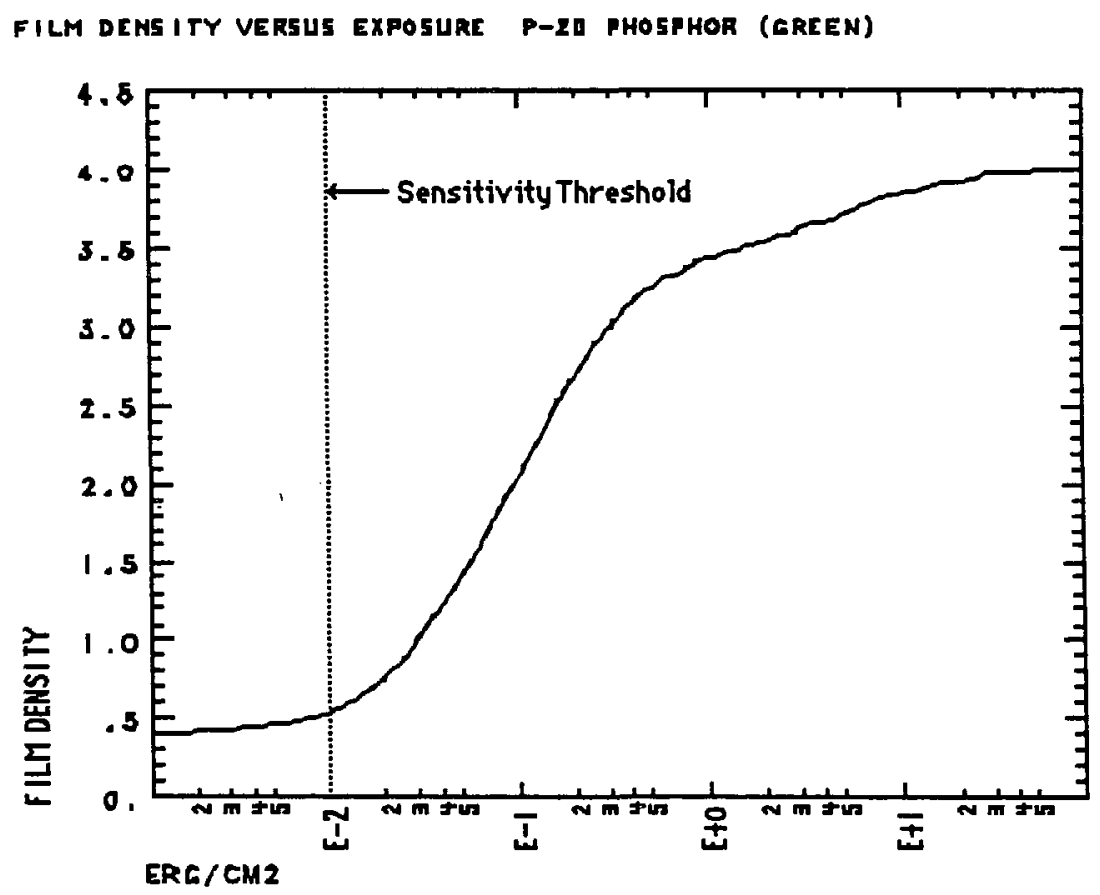

Figure III.D.2 Density versus exposure curve for 2484 film (green light and HOPE 300 processing). 
$f / 2.8$ Nikon. The loss in lens speed was made up for by the increase in image intensity. The video signal from the camera was recorded onto $\frac{3}{4}$ inch video tape. To digitize an image, the tape was played back, and the video signal sent through a digitizer. The digitizer is controiled through a CAMAC interface by a microcomputer, which can write the digitized data onto $3 \frac{1}{2}$ inch disks. The data can later be downloaded from the disks onto the computer system used for the data analysis.

\section{E. Shielding}

A diagram of the camera shielding is shown in figure III.E.1. To unambiguously image the $\mathrm{x}$ rays originating in the camera field of view, the detector must be shielded from strong magnetic fields, high energy $\mathrm{x}$ rays, and fusion-produced neutrons.

The magnetic shielding directly surrounds the detector. The magnetic field at the location of the instrument during an experimental shot is on the order of 1000 Gauss. In order to reduce the field down to a few gauss (the level at which the light intensifiers were designed to operate), materials with different magnetic properties were nested inside each other. Outermost is a box made of $\frac{1}{4}$ inch soft iron. The iron does not have a high magnetic permeability, but it does have a high saturation level, which allows it to attenuate the initial high field. Inside the iron box, separated by $\frac{1}{8}$ inch thick $\mathrm{Al}$ spacers, are $\frac{1}{16}$ inch sheets of high permeability netic and conetic material. The conetic material has the higher magnetic permeability and is therefore nearest to the instrument. The Al, which is non-magnetic, keeps 


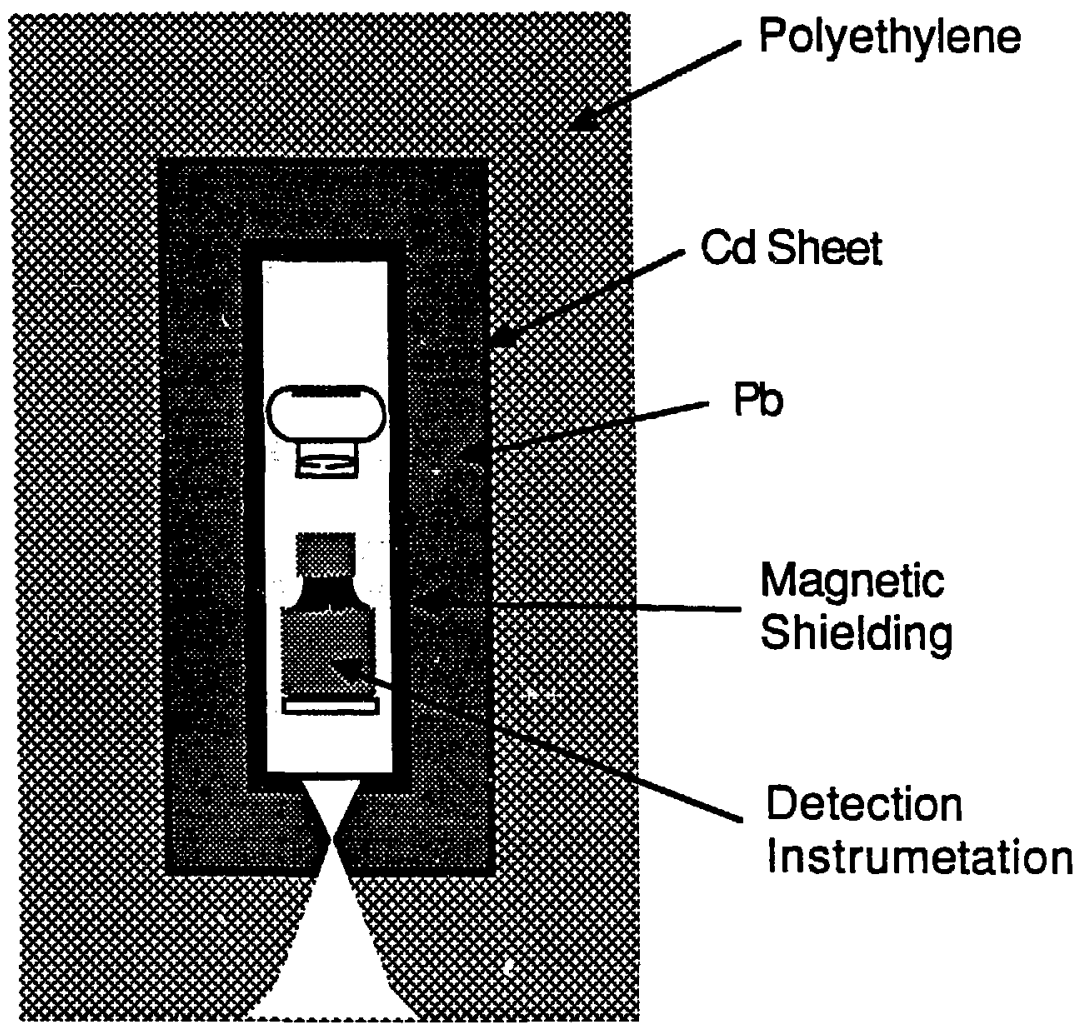

Figure III.E.1. Camera shielding. 
magnetic flux from flowing from one shielding material to the next. There is also a sheet of mu-metal wrapped directly around the intensifiers themselves. Time-resolved measurements made with the CCD camera indicate that the magnetic shielding is sufficient-the image does not distort in any way as the magnetic field varies.

Outside the magnetic shielding is a minimum four inch thickness of lead $\mathrm{Pb}$ ) which essentially limits the $\mathrm{x}$-ray flux to that which passes through the pinhole. The iron and aluminum in the magnetic shielding act as a graded shield for $\mathrm{x}$ rays which come through the pinhole and are not absorbed in the scintillator crystal. A graded shield consists of materials with different atomic numbers layered so that fluorescent radiation excited in one section will be absorbed before it reaches the detector (i.e. $\mathrm{Pb}$ fluorescence is absorbed in the $\mathrm{Fe}$, and Fe fluorescence in the Al). ${ }^{4}$ To eliminate fluorescence off the conical surface of the pinhole nearest the scintillator, a graded shield was made in the form of a cone, consisting of sheets of $\mathrm{Ta}, \mathrm{Cd}, \mathrm{Cu}, \mathrm{Fe}$, and Al.

Neutron shielding forms the outermost layer. This shielding is necessary whenever the neutral beams are running. Neutrons are produced by deuterium-deuterium reactions

$$
\mathrm{D}+\mathrm{D} \rightarrow{ }^{3} \mathrm{He}(0.8 \mathrm{MeV})+\mathrm{n}(2.5 \mathrm{MeV})
$$

both in the region where a neutral beam strikes the machine wall, and also to a lesser extent from the plasma itself. The neutrons are first moderated by 
6 inches of polyethylene, and then absorbed by a Cd sheet. Measurements made with $3 \times 3$ inch $\mathrm{NaI}$ detectors indicate that this amount of shielding is sufficient to effectively eliminate the $2.5 \mathrm{MeV}$ neutron produced background.

\section{F. Uniformity}

The gain of the intensifiers is not spatially uniform. To evaluate this effect, a number of exposures were taken with an x-ray source uniformly illuminating the scintillator. A contour plot and 3 pseudo-3d plots of the measured intensity versus position are shown in figure III.F.1, and an axial and radial slice through the point of peak efficiency are shown in figure III.F.2. There are two major nonuniformities-a peak in roughly the center of the image and a dead region to the left of center (looking at the contour plot). Fortunately the dead region lies in an area of the image shadowed by one of the magnet coils; the peak, however, is in an area where the camera is already most sensitive due to the collimation effects of the pinhole (see section $\mathrm{B}$, chapter $\mathrm{V}$ ). To compensate for the effects of this nonuniformity, the detected signal is weighted by the inverse of the measured two-dimensional efficiency array.

\section{G. Calibration}

The detection system was calibrated with a known radioactive $\mathrm{x}$-ray source. The source emitted $x$ rays at energies of $59.5\left({ }^{241} \mathrm{Am}, 60 \mu \mathrm{Ci}\right)$ and $5.90 \mathrm{keV}\left({ }^{55} \mathrm{Fe}, 85.6 \mu \mathrm{Ci}\right)$, and was collimated with a $\frac{1}{8}$ inch $\mathrm{Pb}$ sheet that had an approximately $2.4 \mathrm{~mm}$ diameter hole drilled in it.. A $3 \times 3 \mathrm{NaI}$ 

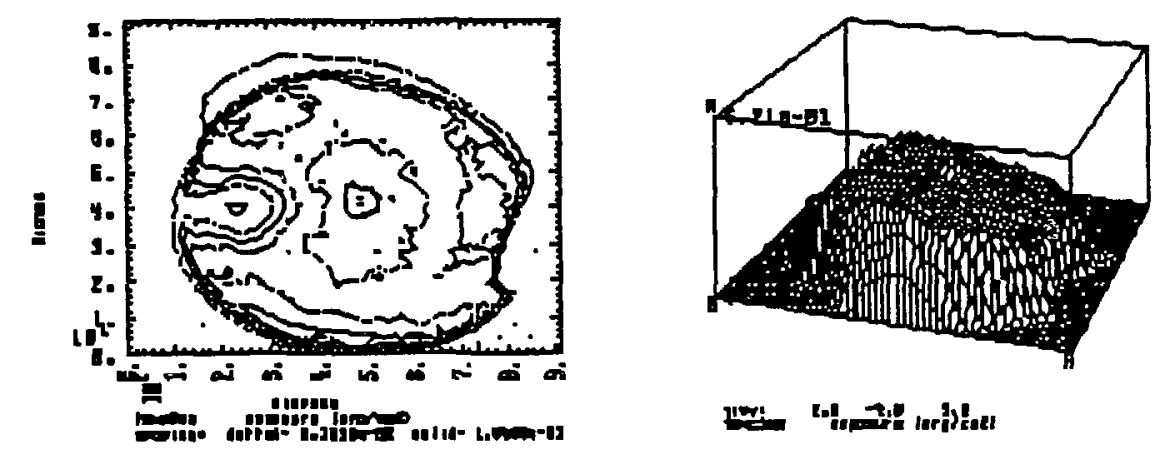

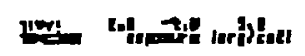
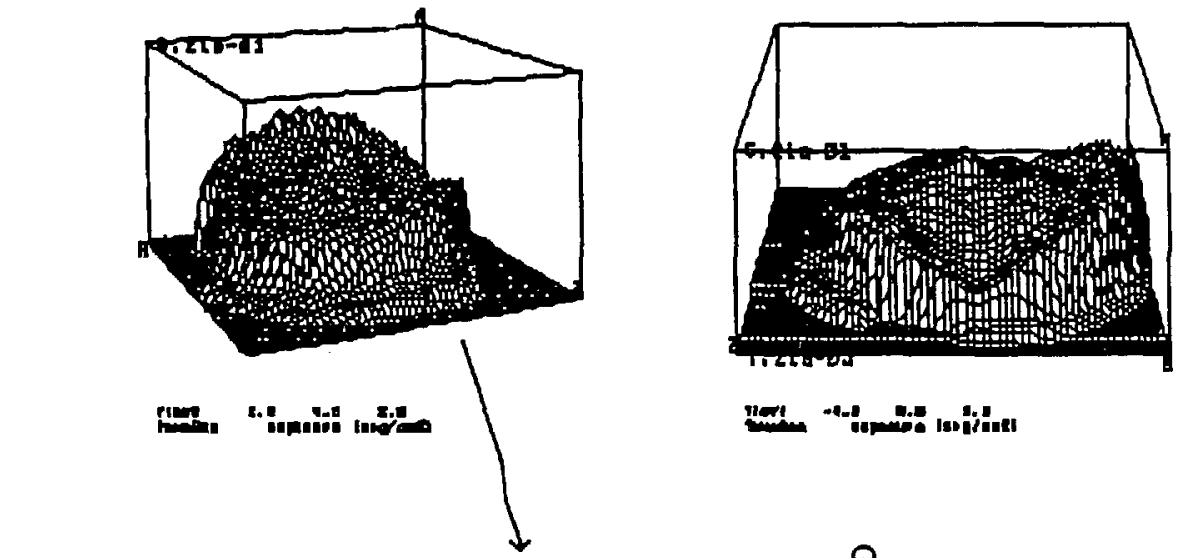

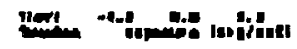

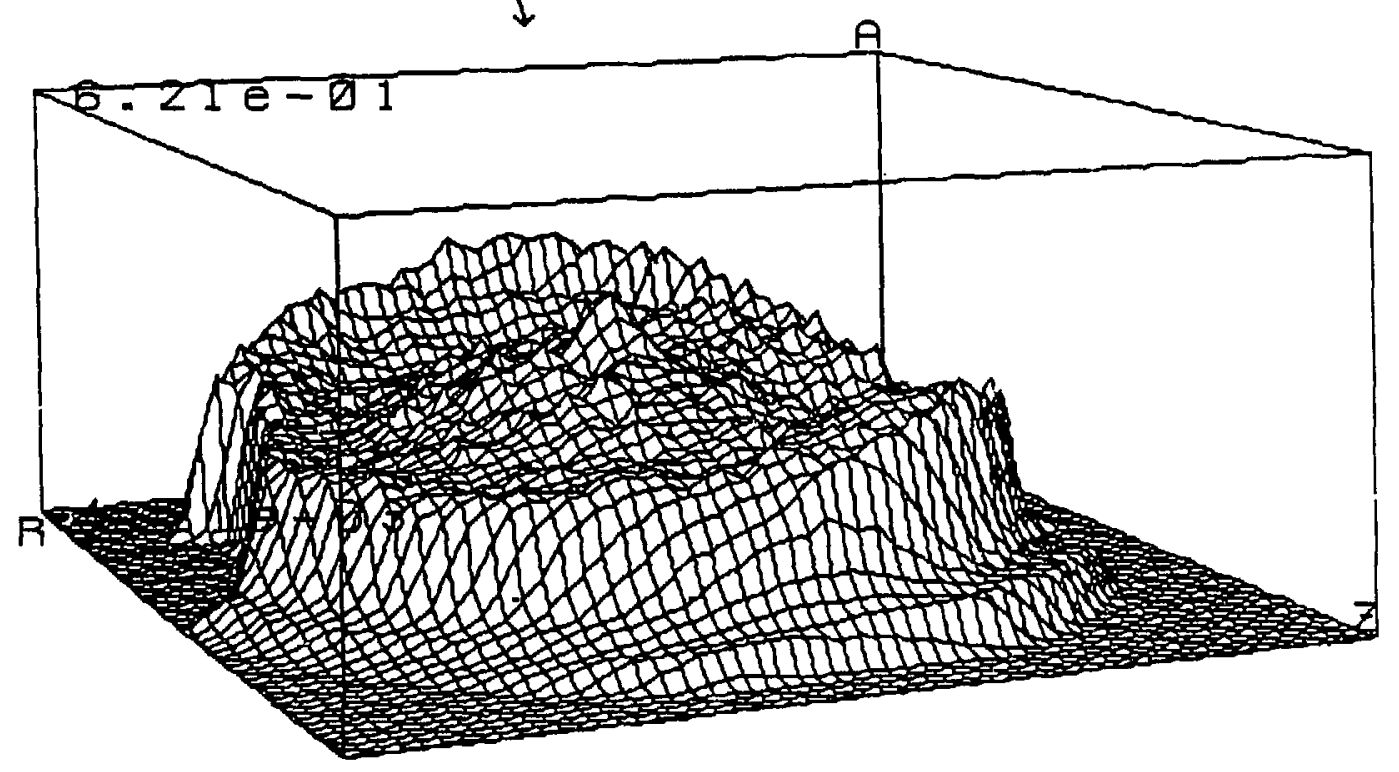

Figure III.F.1. Intensifier nonuniformity. 

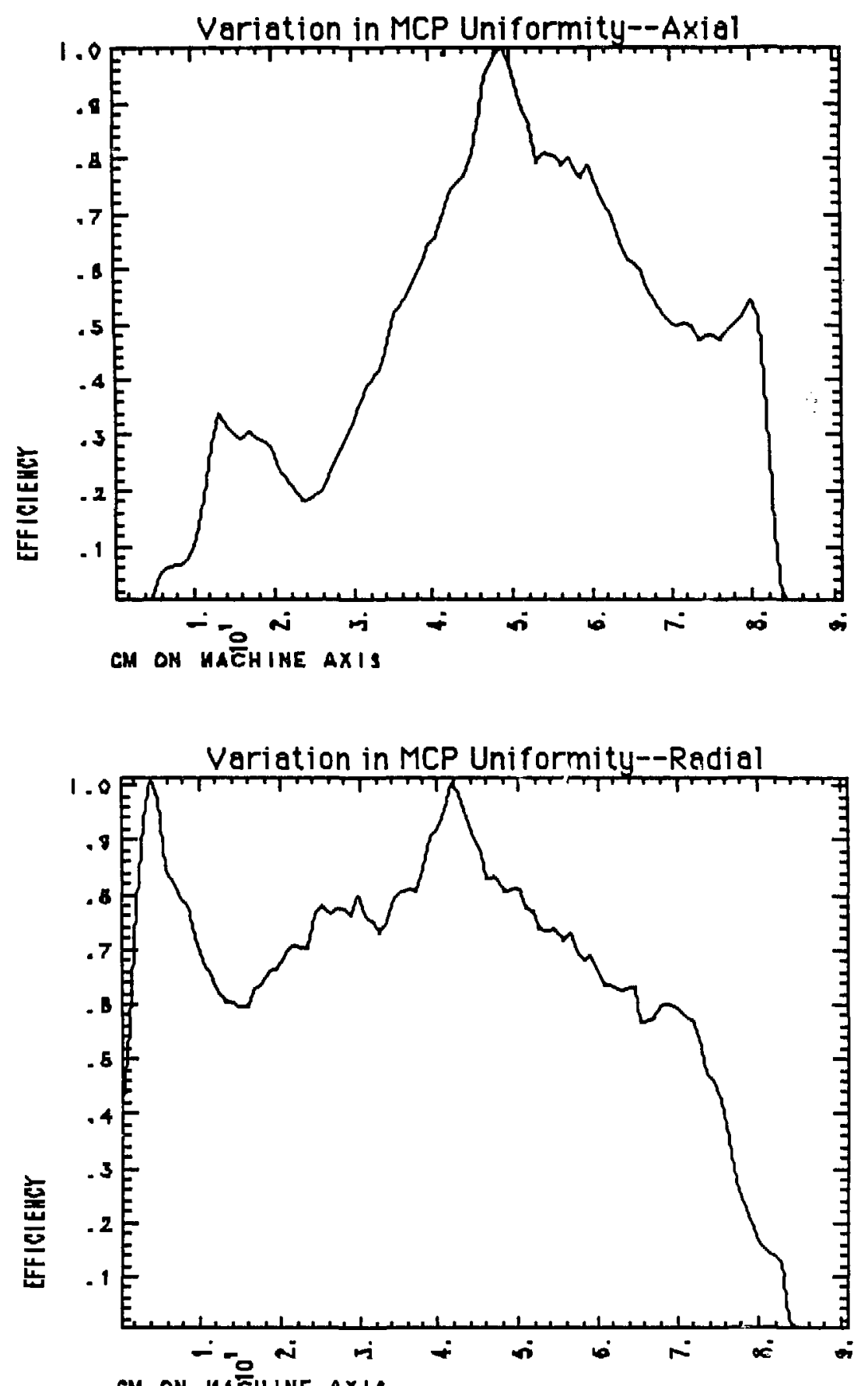

CH DH MACHINE AXIS

Figure III.F.2 Intensifier nonuniformity--axial and radial slices. 
spectrometer system was used to determine the number of $x$ rays which passed through the $\mathrm{Pb}$ sheet collimator.

The source uniformly illuminated a circular region. This was determined from an exposure done with Kodak No Screen x-ray film. Plots showing the film density distribution are given in figure III.G.1. A simulation of the expected instrument response for this source was done and is compared with that actually measured in figure III.G.2. The measured (simulated) response corresponds to the solid (dotted) curve. The fact that the measured response is not as sharp as that predicted by the simulation indicates that other effects, such as the quality of the electron optics in the intensifiers and the sharpness of the lens used tc couple the light to the film, degrade the resolution of the system. Because the amount of energy detected in the wings of the measured image is relatively small, less than $25 \%$ of the total incident, no effort was made to compensate for the effect.

The gain was measured using a number of different apertures and exposure times. The experimental data is plotted in figure III.G.3. The points marked with squares (a) correspond to an aperture setting of $f / 1.4$, the circles $(O)$ to $f / 2.0$, and the triangles $(\Delta)$ to $f / 2.8$. A least-squares fit yields a gain of $35 \pm 3$, with a $y$-intercept of $7.9 \pm .6 \times 10^{-3}$ ergs.

From this overall gain value, the gain of the intensifier chain can be estimated. The efficiency of the scintillator is $11.5 \%$, and total internal reflection condition limits the light which can pass into the fiber optic to $21 \%$. This means that $2.42 \%$ of the energy that is deposited in the scintillator 

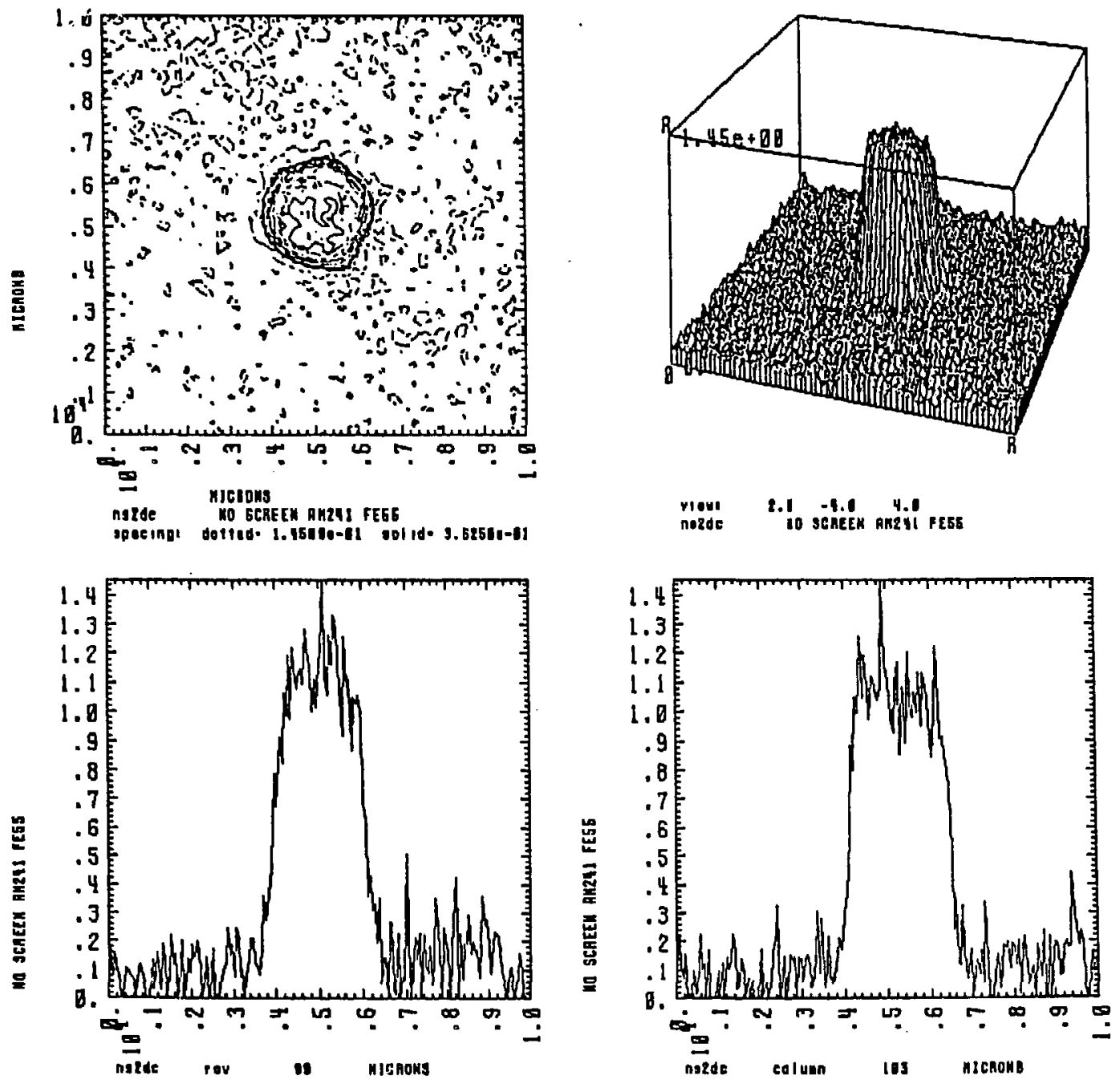

Figure III.G.1. No Screen x-ray film exposure of source used in system calibration. 
Comparison of Measured and Simulated Ltensitles from Source Uaed to Calibrate the Actire Syotem

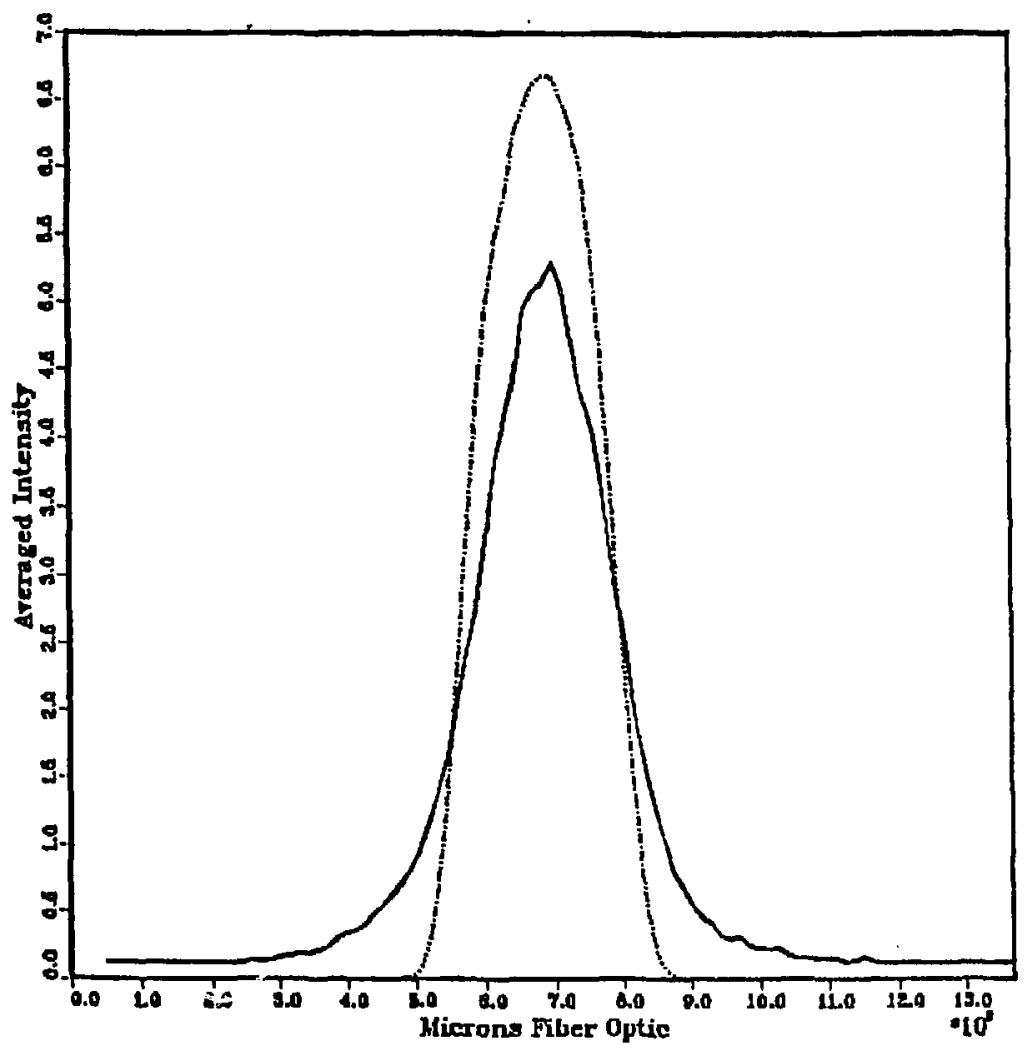

Figure III.G.2. Two curves shown are measured (solid) and simulated (dotted). 


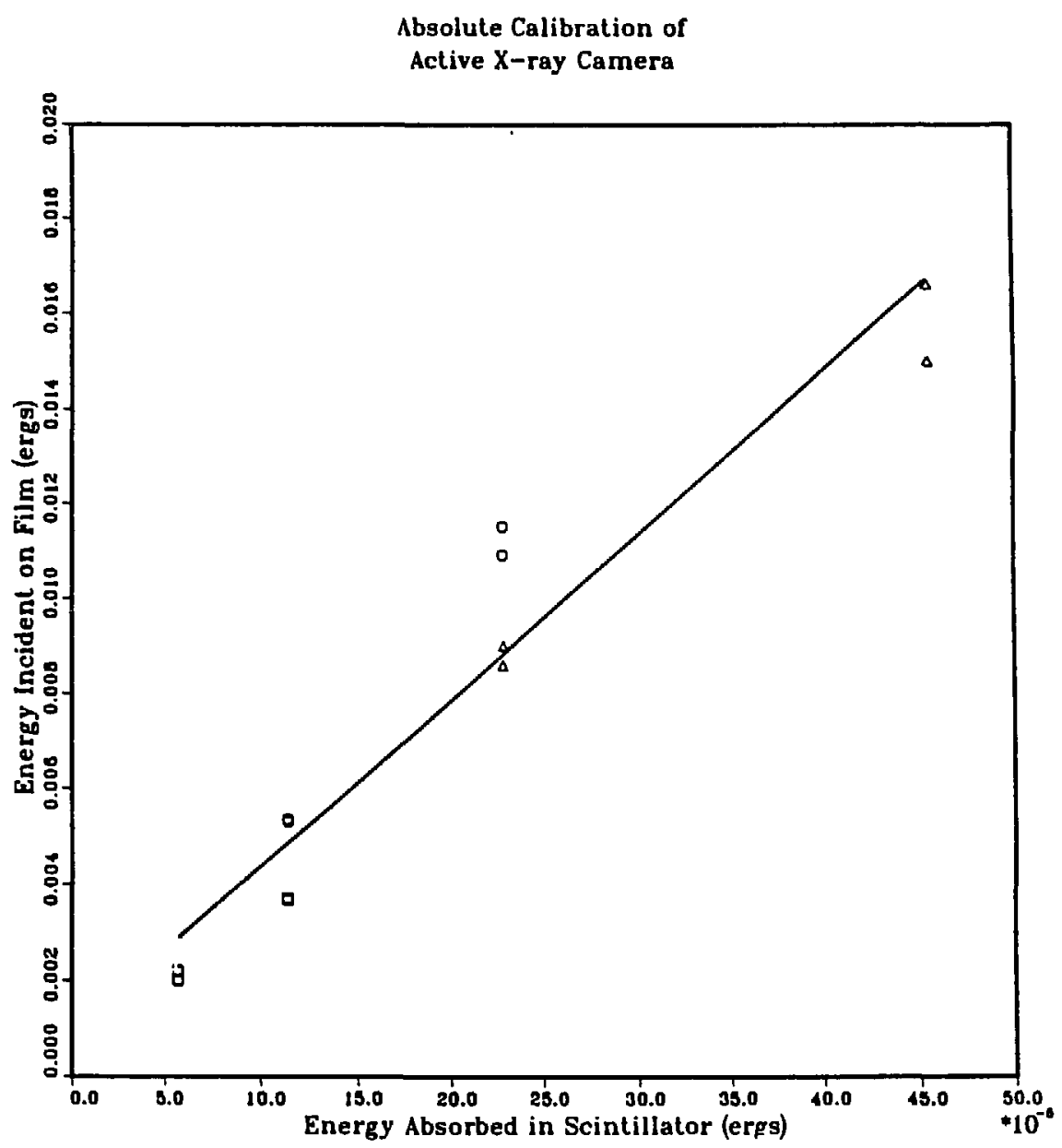

Figure III.G.3. 
reaches the phoiocathode of the first intensifier. The total internal reflection condition at the output of the MCP intensifier (a glass-air interface) allows $12.7 \%$ of the light produced at the phosphor to be transmitted, and the collection efficiency of the lens for an aperture setting of $f / 1.4$ is about $6.0 \%$. Thus the "gain" of everything besides the intensifiers and the 2:1 reducing fiber optic that connects them is $.0184 \%$, which means that experimentally measured that the gain of the intensifier chain is $1.9 \times 10^{5}$.

This intensifier gain is roughly what one might expect. Typical diode intensifier and MCP gains are typically 50 and 40,000 respectively. Because of imperfect alignment at the fiber optic connections, $80 \%$ of the light will be transmitted at each of the three interfaces (scintillator to diode, diode to reducing fiber optic, and reducing fiber optic to MCP), for an overall efficiency of about $50 \%$. Also, the transmission efficiency of the 2:1 reducer is approximately $20 \% .{ }^{5}$ Multiplying these four factors together, the estimated gain is $2.0 \times 10^{5}$, in good agreement with the measured value.

Because the camera has been absolutely calibrated, definite statements about radial electron losses (as evidenced by thick target emissions) and also comparisons of camera data with the line-averaged emissivities measured with the $\mathrm{x}$-ray spectrometers are possible (see chapter VII). The volume $\mathrm{x}$-ray emissivity, usually had to be on the order of a few $\mathrm{ergs} /\left(\mathrm{cm}^{3}-\mathrm{sec}\right)$, as determined from the camera geometry and the above calibration, for a reasonably good image to be acquired in $\frac{1}{60}$ of a second. 


\section{Chapter IV}

\section{System Resolution}

\section{A. Introduction}

In practice, the resolution of the camera is limited by three factors: (1) the pinhole diameter, (2) the transmission of the pinhole, and (3) the thickness of the scintillator in which the $\mathrm{x}$ ray is absorbed. All of these impose limitations on the resolution that is possible, so in order to optimize the performance of the camera one needs to consider the effects of each.

\section{B. Pinhole Size}

Consider the pinhoie imaging system shown in figure IV.B.1. The distance from the machine axis to the pinhole, $L$, is determined by the machine structure, thus the source-pinhole distance is fixed. Given the size of the source and the diameter of the detector used to detect the image, the maximum pinhole-detector distance, $l$, is also determined. The ratio of these two distances determines the system magnification, $M$. For the values

$$
\begin{gathered}
L=262.3 \mathrm{~cm} \\
l=15.7 \mathrm{~cm}
\end{gathered}
$$

the magnification is

$$
M=16.7
$$

This means that $1 \mathrm{~mm}$ at the detector plane corresponds to $1.67 \mathrm{~cm}$ on the source. 


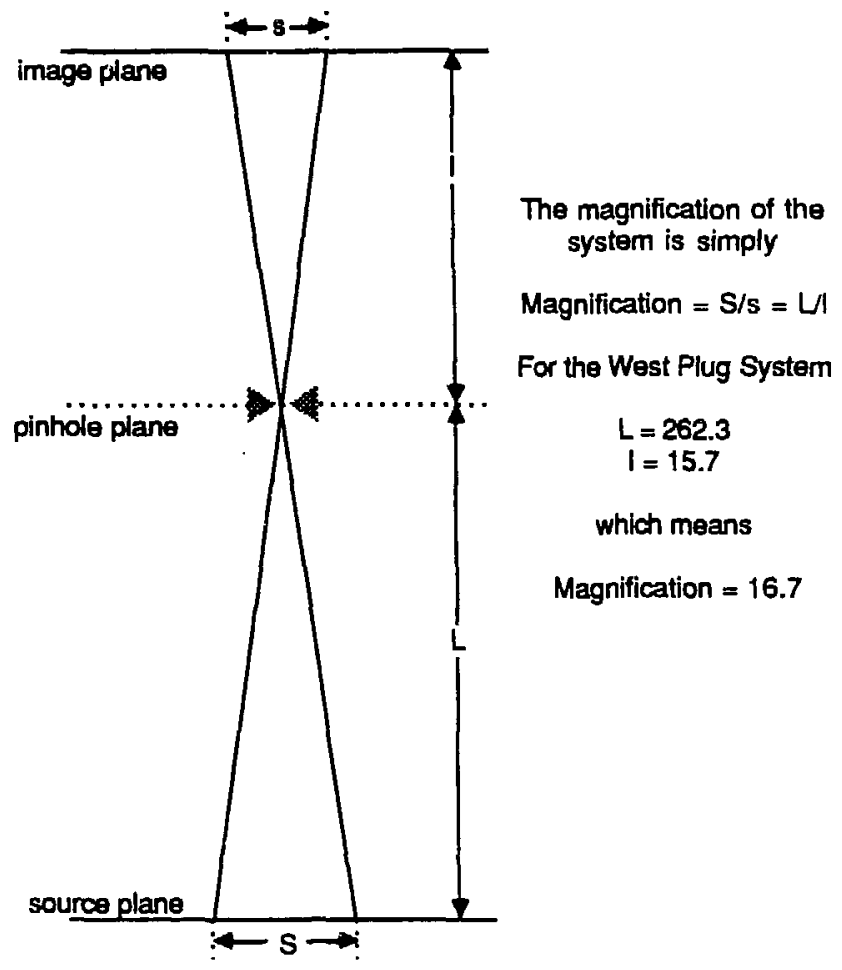

Figure IV.B.1. Magnification of a pinhole imaging system. 
The pinhole diameter affects both the signal level and the number of resolution elements in the image. When the source-pinhole distance is large compared to the pinhole diameter, the solid angle subtended by the pinhole at the source is proportional to the pinhole diameter squared. Also, the numbei of resolution elements per row of the image, $N$, increases as the diameter of the detector, $\Delta$, divided by the pinhole diameter, $d$. The number of resolution elements per row of the image is given by

$$
N=\frac{\Delta}{d}\left(1+\frac{l}{L}\right)
$$

where all the variables have been defined above in this section and are depicted in figure IV.B.2. Note that unless $l \sim L, N$ is determined solely by the ratio of $\Delta$ to $d$.

\section{Pinhole Transmission}

Another issue that affects the system resolution is the fact that the pinhole is not completely opaque-that is some $\mathrm{x}$ rays are transmitted without being imaged. In practice this means that the radius of the pinhole cannot be chosen to be much greater than its thickness, and for this reason collimation effects become important (see section B, chapter V).

The design of the pinholes that were used is shown in figure IV.C.1. The ratio of the radius, $r$, to the half length of the pinhole cylindrical section, $l$, is determined by the desired field of view and the amount of collimation that can be tolerated. For the values

$$
\begin{aligned}
r & =1.4 \mathrm{~mm} \\
l & =5.32 \mathrm{~mm}
\end{aligned}
$$




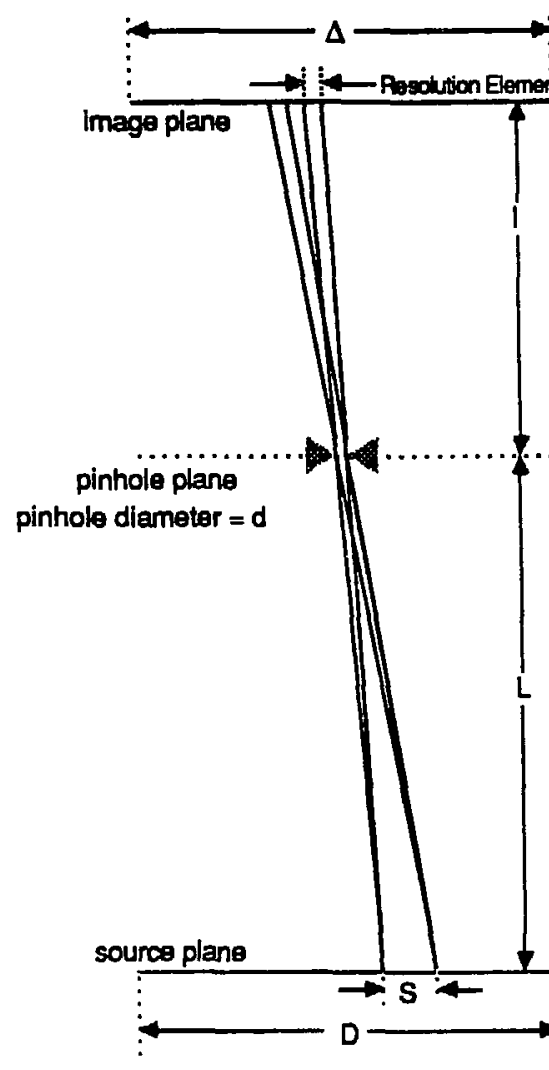

The number of resolution elements is given by the formula:

$N=(\Delta / d,(1+1 / L)$

For the West Plug system

$\Delta=4.0 \mathrm{~cm}$

$\mathrm{I}=15.7 \mathrm{~cm}$

and $L=262.3 \mathrm{~cm}$

So that $\mathbf{N}$ is given by

$N=37.74 / d$

Where $\mathrm{d}$ is given in $\mathrm{mm}$.

Figure IV.B.2. Resolution of a pinhole imaging system. 
their ratio, call it $\alpha$, is

$$
\alpha=.263 \text {. }
$$

For this value of $\alpha$, the effective area of the pinhole seen by a point on the axis of the machine $(262 \mathrm{~cm}$ from the pinhole), and $20 \mathrm{~cm}$ off the axis of the pinhole is reduced from its peak value by $40 \%$. (This reduction is discussed in chapter $\mathrm{V}$, section $\mathrm{B}$.)

Once $\alpha$ has been determined, $r$ must be chosen so that the transmission of the incident $x$ rays is as uniform as possible over the energy range of interest. To understand how well or consistently $\mathrm{x}$ rays of different energies are imaged, consider a monochromatic beam, intensity $I_{0}$, traveling parallel to the axis of a pinhole such as the one shown in figure IV.C.1. After the $\mathrm{x}$ rays have traveled through the pinhole a distance $x$, they are attenuated in accordance with Beer's Law and the intensity, $I$, at that position is given by

$$
I=I_{0} \exp (-\mu \rho x)
$$

where $\mu$ is the mass attenuation coefficient and $\rho$ is the density of the pinhole material. Because the pinhole thickness varies as a function of the distance from the pinhole axis, $r^{\prime}$, so will the transmission. In the pure cone limit $(r \rightarrow 0)$

$$
I\left(r^{\prime}\right)=I_{0} \exp \left(-\frac{2 \mu \rho r^{\prime}}{\alpha}\right)
$$




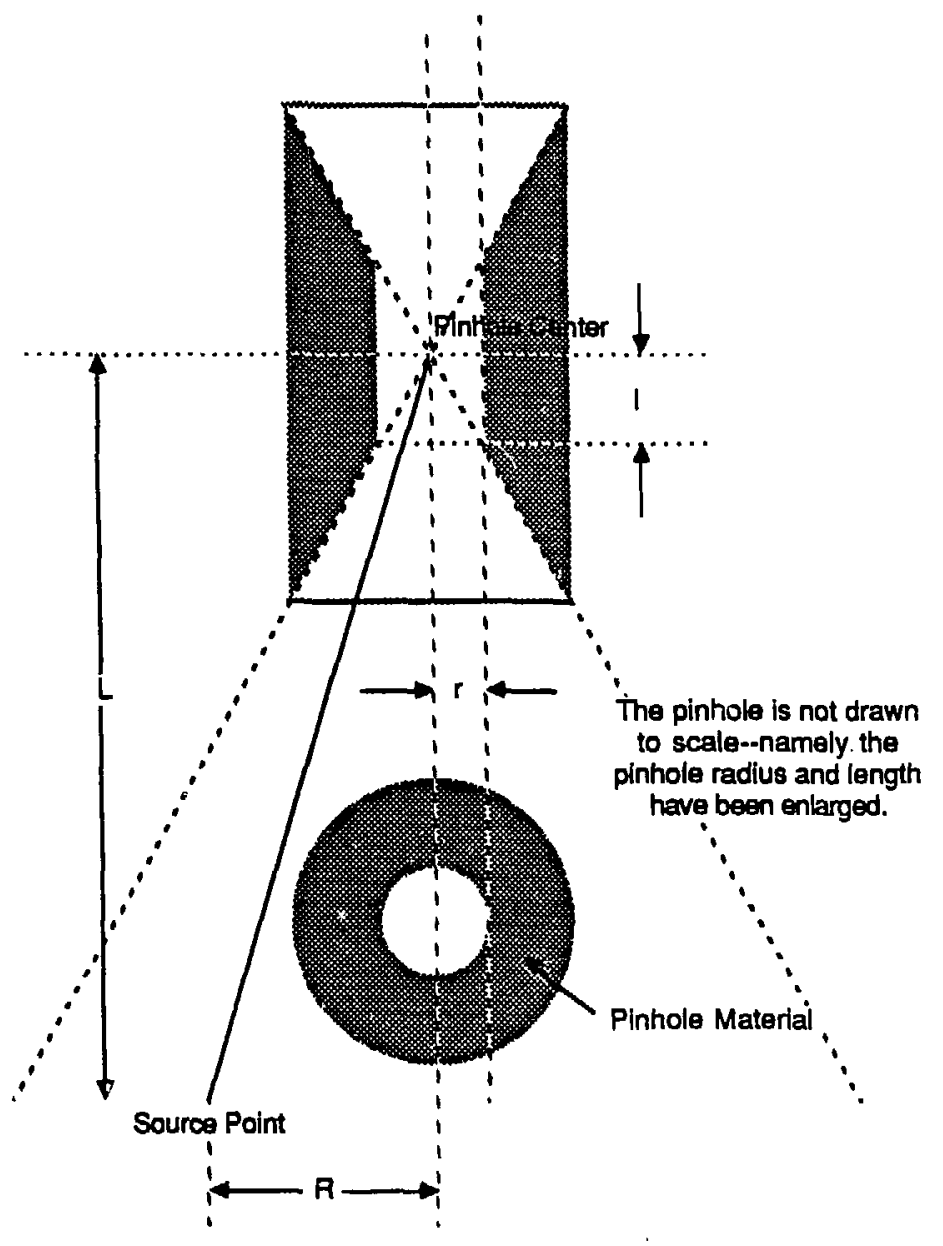

Figure IV.C.1. Pinhole design. 
One can define an eiective pinhole transmission cross-section, $A_{\text {cross }}$, in the following way

$$
\begin{aligned}
A_{\text {cross }} & =\pi \int_{0}^{\infty} d r^{\prime} r^{\prime} I\left(r^{\prime}\right) / I_{0} \\
& =2 \pi r_{\text {cone }}^{2}
\end{aligned}
$$

where

$$
\tau_{\text {cone }}=\frac{\alpha}{2 \mu \rho} .
$$

From this it car be seen that in the pure cone limit the effective $r$ of the pinhole goes down as one over $\mu \rho$ and increases linearly with $\alpha$. Since $\mu$ varies as a function of $\mathrm{x}$ ray energy, so will $A_{\text {cross }}$.

It is desirable that $A_{\text {cross }}$ vary little for the x-ray energy range of interest. For the scintillators used, the high energy absorption limit is on the order of $200 \mathrm{keV}$, so ideally the $A_{\text {cross }}$ of the pinhole should be constant below that energy. When $r \neq 0$, the expression for $A_{\text {cross }}$ becomes

$$
\begin{aligned}
A_{\text {cro.ss }} & =\pi\left(r^{2}+\int_{r}^{\infty} d r^{\prime} r^{\prime} I\left(r^{\prime}\right) / I_{0}\right) \\
& =\pi\left[r^{2}+2 r_{\text {cone }}^{2}\left(1+\frac{r}{r_{\text {cone }}}\right) \exp \left(-\frac{r}{r_{\text {cone }}}\right)\right] .
\end{aligned}
$$

Figure IV.C.2 shows how $A_{\text {cross }}$ varies for lead $(\mathrm{Pb})$ and uranium (U) pinholes at $\mathrm{x}$-ray energies above $100 \mathrm{keV}$. The three curves on each plot correspond to $\mathrm{r}$ values of $0, .05$, and $.14 \mathrm{~cm}$. Although the $\mathrm{U}$ shows less of an increase in $A_{c r o s s}$ at high energies, the uranium pinhole that we tried to use had a background emission spectrum associated with it, thought to result from primary and/or secondary excitation due to $\alpha$ particle emission from thorium impurities. This background can be suppressed by covering the uranium with a sufficiently thick sheet of $\mathrm{Pb}$, but this has not been implemented 
as yet. Since, according to the calculations, $\mathrm{Pb}$ should perform well up to $\mathrm{x}$-ray energies of $200 \mathrm{keV}$ for $r=.05 \mathrm{~cm}$ and $300 \mathrm{keV}$ for $r=.14 \mathrm{~cm}, \mathrm{~Pb}$ pinholes were used for the experimental measurements.

The calculations for the $\mathrm{Pb}$ pinholes are born out by the results of raytracing runs shown in figure IV.C.3. Shown are a contour, a pseudo-3D, and a horizontal lineout plot (passing through the maximum) of the $\mathrm{x}$-ray intensity that would be incident on the front of the scintillator. X-ray energies of 10 , 200 , and $400 \mathrm{keV}$ were used (only the 200 and $400 \mathrm{keV}$ cases are shown in the figure, however), and the pinhole diameter was $1 \mathrm{~mm}$. There is little difference between the 10 and $200 \mathrm{keV}$ cases, but the pinhole is beginning to lose its sharpness at an $\mathrm{x}$-ray energy of $400 \mathrm{keV}$. Just how much the variation of $A_{\text {cross }}$ will affect the resolution of the system depends on the scintilli' ? efficien:y and the $x$-ray spectrum of the source above $200 \mathrm{keV}$.

\section{Scintillator Thickness}

One more factor that affects the resolution of the imaging system is the type of scintillator that is used. The size of the spot that will be produced by the absorption of a single $\mathrm{x}$ ray depends on two things-the index of refraction mismatch between the scintillator and the fiber optic, and the location where the $\mathrm{x}$ ray is absorbed in the crystal.

When an $\mathrm{x}$ ray is absorbed in a scintillator, a fraction of its energy (for CsI about $11 \%$ ) is radiated at visible or near ultraviolet wavelengths isotropically from that location. If the energy of the absorbed $\mathrm{x}$ ray is $E_{0}$ 

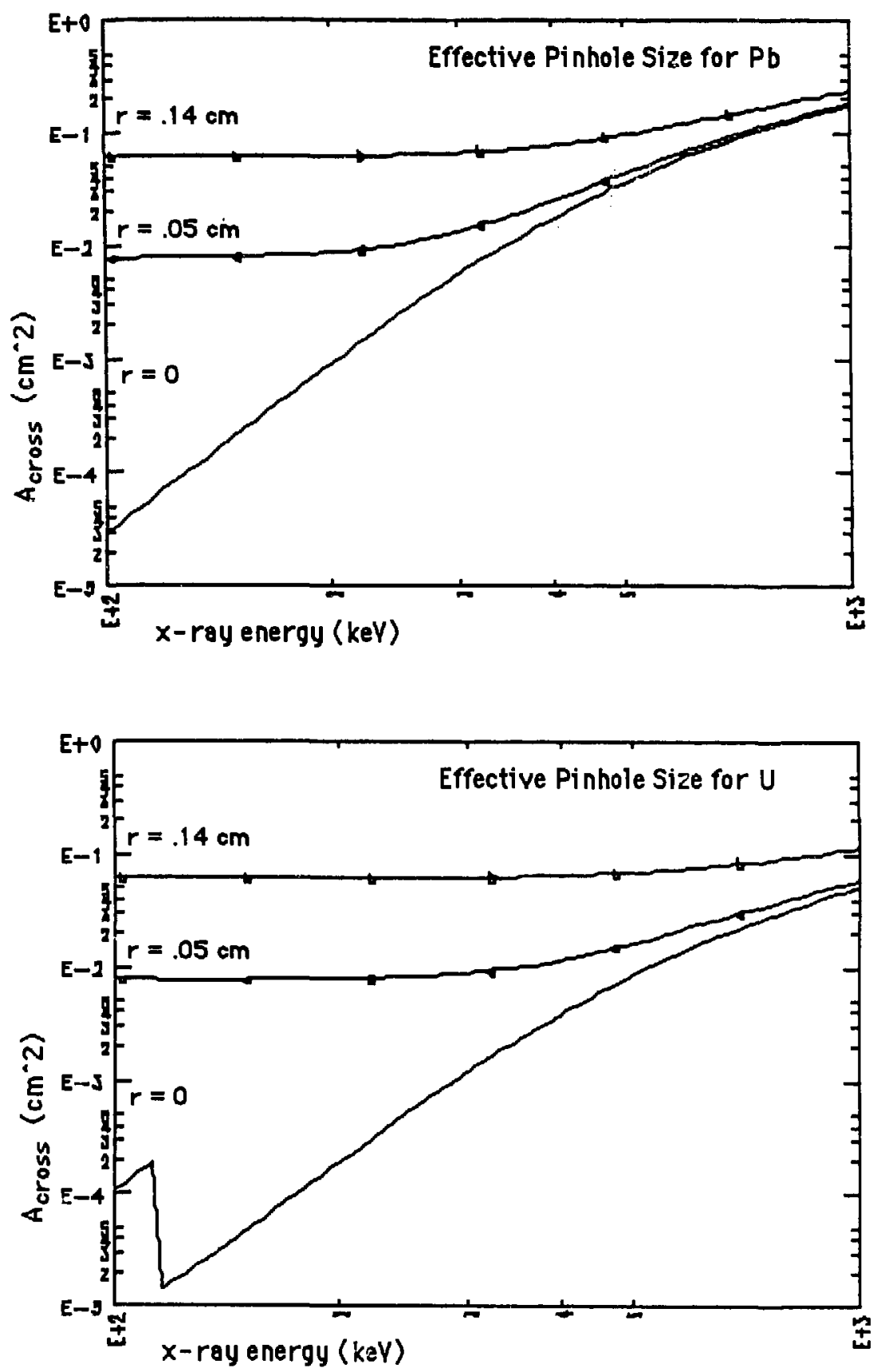

Figure IV.C.2. Effective pinhole cross-sections for $\mathrm{Pb}$ and $\mathrm{U}$. 
Photon Energy of $200 \mathrm{keV}$
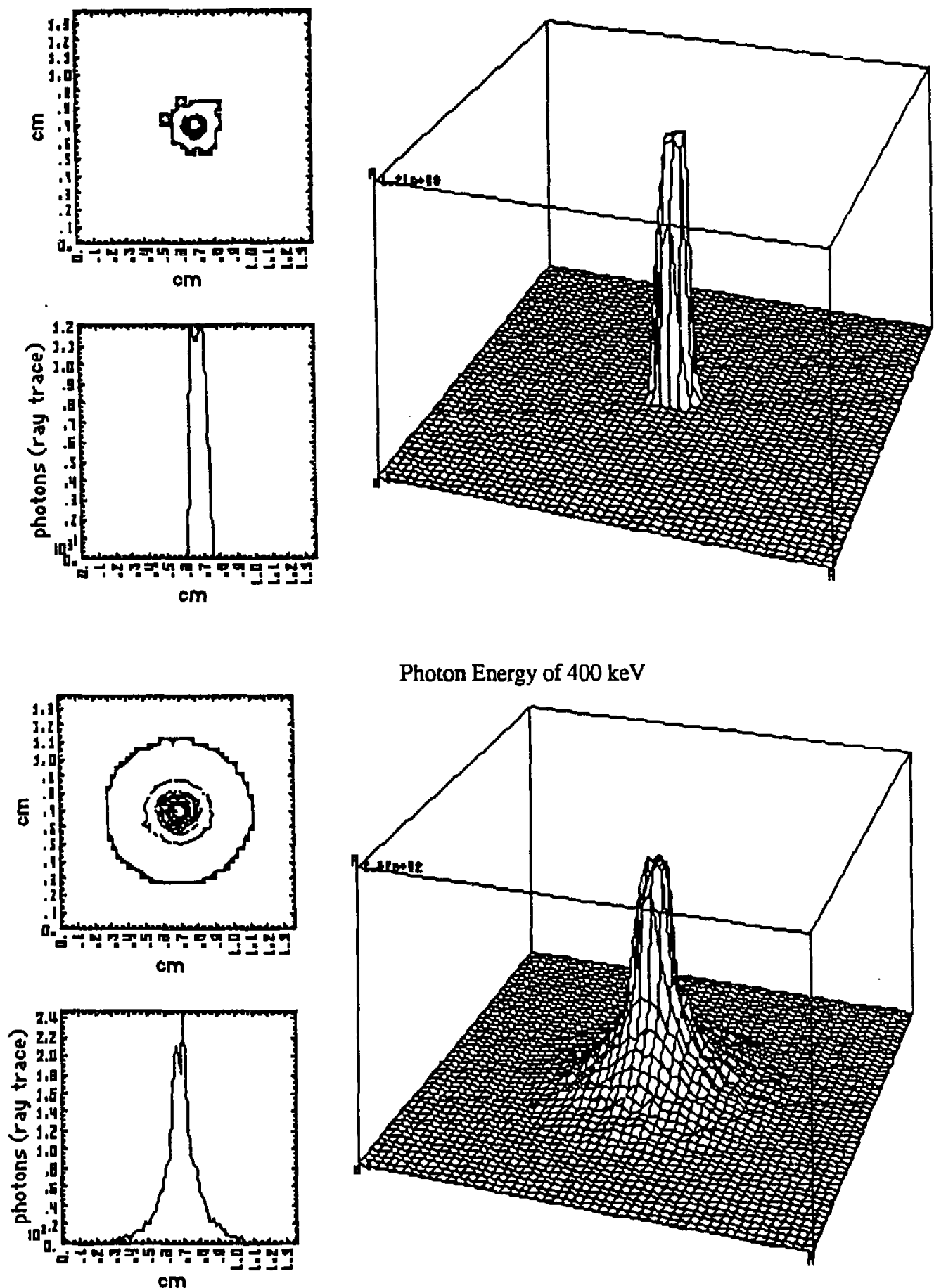

Figure IV.C.3. Degradation of pinhole sharpness ât high photon energies. 
and the scintillation efficiency is $\eta_{s}$, the energy converted to optical light, $E_{s}$, is given by

$$
E_{s}=\eta_{s} E_{0}
$$

This light passes through the scintillator/fiber optic interface; the maximum angle at which he light can be incident on this interface and still be transmitted is given by the angle of total internal reflection, $\phi_{\max }$. If $n_{g}$ and $n_{f o}$ are the indices of refraction for the scintillator and fiber optic, respectively, then according to Snell's law

$$
\phi_{m a x}=\arcsin \left(\frac{n_{j !}}{n_{r}}\right) .
$$

The diameter of the spot, $d$, (where it is cut off due to total internal reflection) is given by

$$
d=2 b \tan \phi_{\max }
$$

where $b$ is the distance between the absorption location and the scintillator/fiber optic interface. Figure IV.D.1 shows how $d$ varies with $\phi_{\max }$, which corresponds to two different scintillators coupled to the same fiber optic. Figure IV.D. 2 shows how $d$ varies linearly with $b$, the absorption location of the $x$ ray. An $x$ ray with a short mean free path in the crystal will typically produce a larger spot than an $\mathrm{x}$ ray with a long one.

Although $d$ is the diameter at which light can no longer be transmitted, it is not accurate to assume that the intensity is uniform out to that point and then drops abruptly. The way in which the intensity falls off with the 


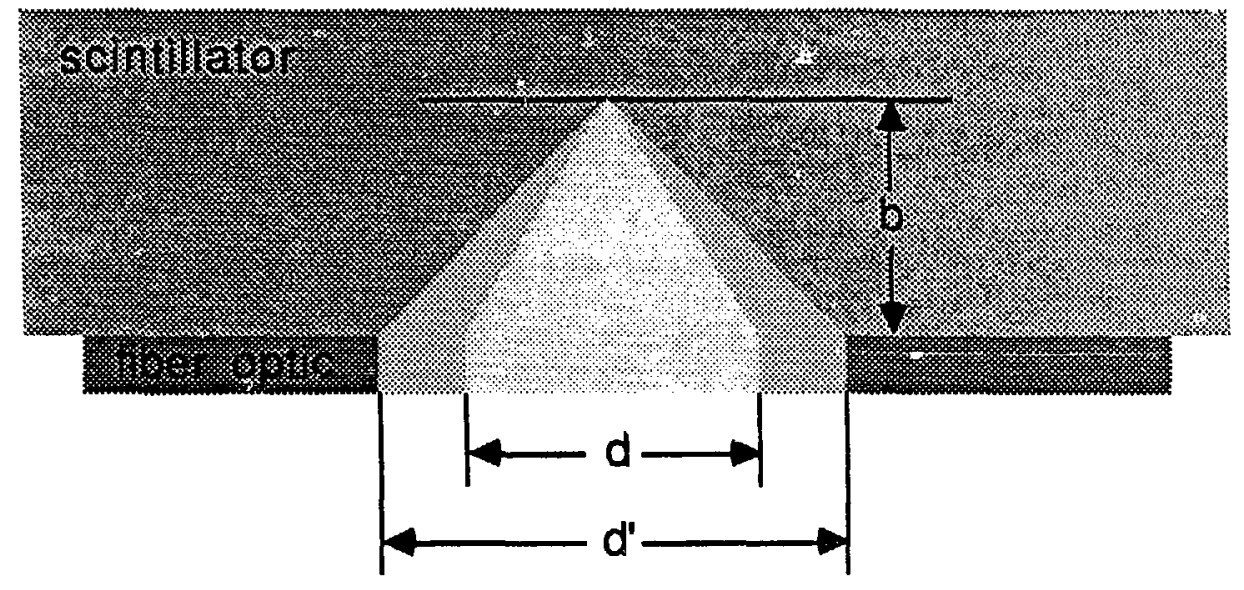

For a given absorption location, $b$, the spot size, $d$, depends on the ratio of the index of refraction of the scintillator to that of the fiber optic.

Figure IV.D.1. Spot size dependence on angle of total internal reflection. 


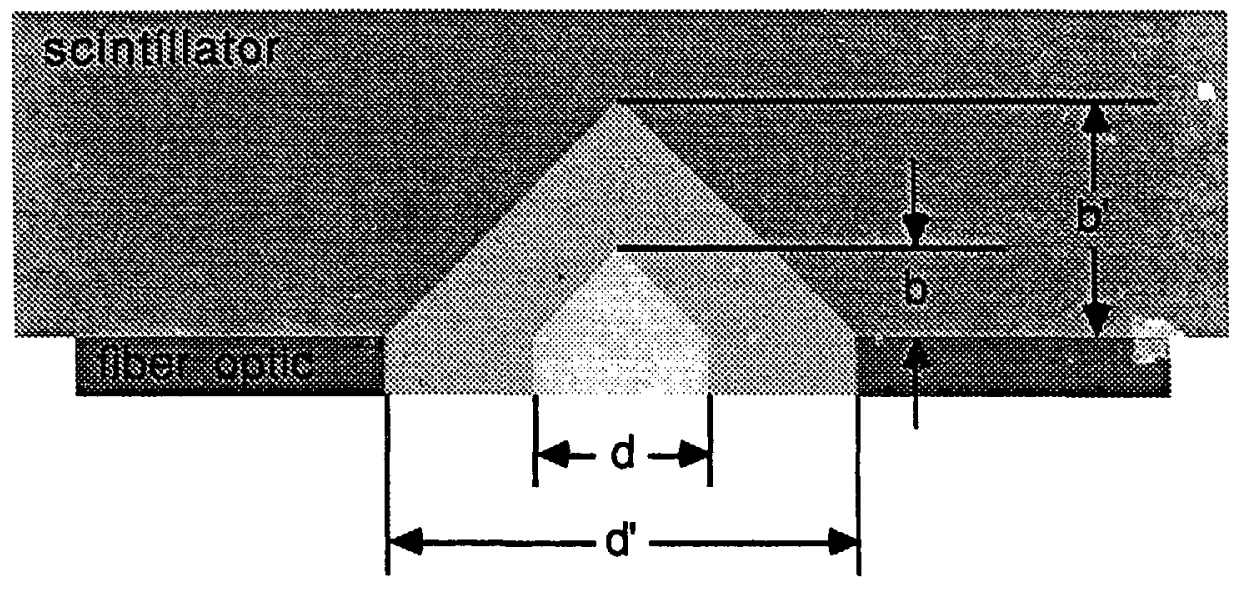

For a given scintillator/fiber optic combination, the ratio of spot size, $d$, to absorption location, $b$, is a constant. The constant is determined by the ratio of the index of refraction of the fiber optic to that of the scintillator.

Figure IV.D.2. Spot size dependence on x-ray absorption location. 
distance from the absorption location in the plane of the interface, $r$, for a given $b$, is

$$
I(r)=\frac{E_{s}}{2 b^{2}}\left[1+\left(\frac{r}{b}\right)^{2}\right]^{-\frac{3}{2}}
$$

and the energy deposited within radius, $r$, is given by

$$
E(r)=\int_{0}^{r} d r^{\prime} r^{\prime} I\left(r^{\prime}\right)=\frac{E_{s}}{2}\left\{1-\left[1+\left(\frac{r}{b}\right)^{2}\right]^{-\frac{1}{2}}\right\} .
$$

$E_{s}$ is the total amount of energy scintillated. The ratio of the intensity at $r=\frac{1}{2} d$ (defined as cut-off) to that at $r=0$ (peak) is $\cos ^{3} \phi_{\max }$. For a $\mathrm{CsI} / \mathrm{glass}$ interface, $\phi_{\max }=54.6^{\circ}$, so the ratio of intensity at cut-off to the peak value is .19. Note also that $21 \%$ of $E_{s}$ is deposited within a radius of $\frac{1}{2} d$.

To determine the shape of a spot that would be produced by an $\mathrm{x}$ ray of given energy, one needs to average over all the possible absorption locations in the crystal. For a beam of monoenergetic $x$ rays, the intensity at any point in the crystal is given by equation (IV.C.1). Defining a mean free path in the scintillator, $\lambda$, as

$$
\lambda=\frac{1}{\mu \rho}
$$

the amount of energy deposited between $b$ and $b+d b$ is

$$
d E=-E_{0} \exp \left(\frac{b-b_{\max }}{\lambda}\right) \frac{d b}{\lambda}
$$

where $E_{0}$ is the total x-ray energy incident. So now $I(r)$ can be determined by summing the contributions at all the possible absorption locations,

$$
I(r)=\int_{r \cot \phi_{m, 1}}^{b_{r n u x}} \frac{\eta_{s} E_{0}}{2 b^{2}} \exp \left(\frac{b-b_{\max }}{\lambda}\right)\left[1+\left(\frac{r}{b}\right)^{2}\right]^{-\frac{3}{2}} \frac{d b}{\lambda}
$$


The lower limit of the integral is due to the total internal reflection cut-off.

It is worthwhile to look at both the short and long mean free path limits of equation (IV.D.3). In the short mean free path limit,

$$
\lim _{\lambda / b_{\max } \rightarrow 0} \frac{\exp \left(\frac{b-b_{\max }}{\lambda}\right)}{\lambda}=\delta\left(b-b_{\max }\right)
$$

and $I(r)$ goes to equation (IV.D.1), replacing $b$ with bmax. While in long mean free path limit

$$
\lim _{\lambda / b_{\max } \rightarrow \infty} \frac{\exp \left(\frac{b-b_{\max }}{\lambda}\right)}{\lambda}=\frac{1}{\lambda} .
$$

By integrating equation (IV.D.3) in this limit, one obtains

$$
I(r)=\frac{E_{s}}{2 \lambda}\left[\frac{\sin \phi_{\max }}{r}-\left(r^{2}+b_{\max }^{2}\right)^{-\frac{1}{2}}\right]
$$

and the energy deposited within radius, $r$, is

$$
E(r)=\frac{E_{s}}{2 \lambda}\left\{1+\frac{r}{b_{\max }} \sin \phi_{\max }-\sqrt{1+\left(\frac{r}{b_{\max }}\right)^{2}}\right\} .
$$

This is theoretically the most peaked intensity profile one can obtain. Note that $I$ is identically zero at $r=b_{\max } \tan \phi_{\max }$, instead of the finite value found in the short mean free path limit.

Simulations have been done to verify the predicted $I(r)$ in both limits. The results are shown in figure IV.D.3, for the short and long mean free path cases. For each figure a contour and pseudo-3D plot of $I$ is shown, as well as a horizontal slice threigh the point where the intensity is a maximum. The 
simulations agree with the intensity profiles predicted by equations (IV.D.1) and (IV.D.4).

In order to be able to characterize $I(r)$ by a single parameter, it is useful to define $d_{h a l f}$ as the diameter within which half of the incident energy is deposited. For the short mean free path case, $d_{\text {half }}$ can be determined from equation (IV.D.2),

$$
\frac{d_{h a l f}}{b_{\max }}=2 \sqrt{\frac{4}{\left(1+\cos \phi_{\max }\right)^{2}}-1} .
$$

Note that as $\phi_{\max } \rightarrow 0, d_{\text {half }} \rightarrow 0$, and as $\phi_{\max } \rightarrow \frac{\pi}{2}, d_{\text {half }} \rightarrow 2 \sqrt{3} b_{\max }$. For the long mean free path case

$$
\frac{d_{h a l f}}{b_{\max }}=\frac{\sin o_{\max }\left(1+\cos \phi_{\max }\right)-\sqrt{1+2 \cos \phi_{\max }-3 \overline{\cos ^{2} \phi_{\max }}}}{\cos ^{2} \phi_{\max }}
$$

For this case, as $\dot{\phi}_{\max } \rightarrow \frac{\pi}{2}, d_{\text {half }} \rightarrow \frac{3}{2} b_{\max }$. For a CsI/glass interface,

$$
\begin{aligned}
\lim _{\lambda / b_{m i x} \rightarrow 0} \frac{d_{\text {half }}}{b_{\text {max }}} & =1.55 \\
\lim _{\lambda / b_{m i x} \rightarrow \infty} \frac{d_{\text {half }}}{b_{\text {max }}} & =.638 .
\end{aligned}
$$

This means $d_{\text {half }}$ varies by a factor of 2.44 from one limit to the other, and that the best $d_{\text {half }}$ obtainable is .638 times the crystal thickness.

The parameter $d_{\text {half }}$ can be calculated as a function of energy by performing the numerical integration

$$
E(r)=\int_{0}^{r} d r^{\prime} r^{\prime} I\left(r^{\prime}\right)
$$



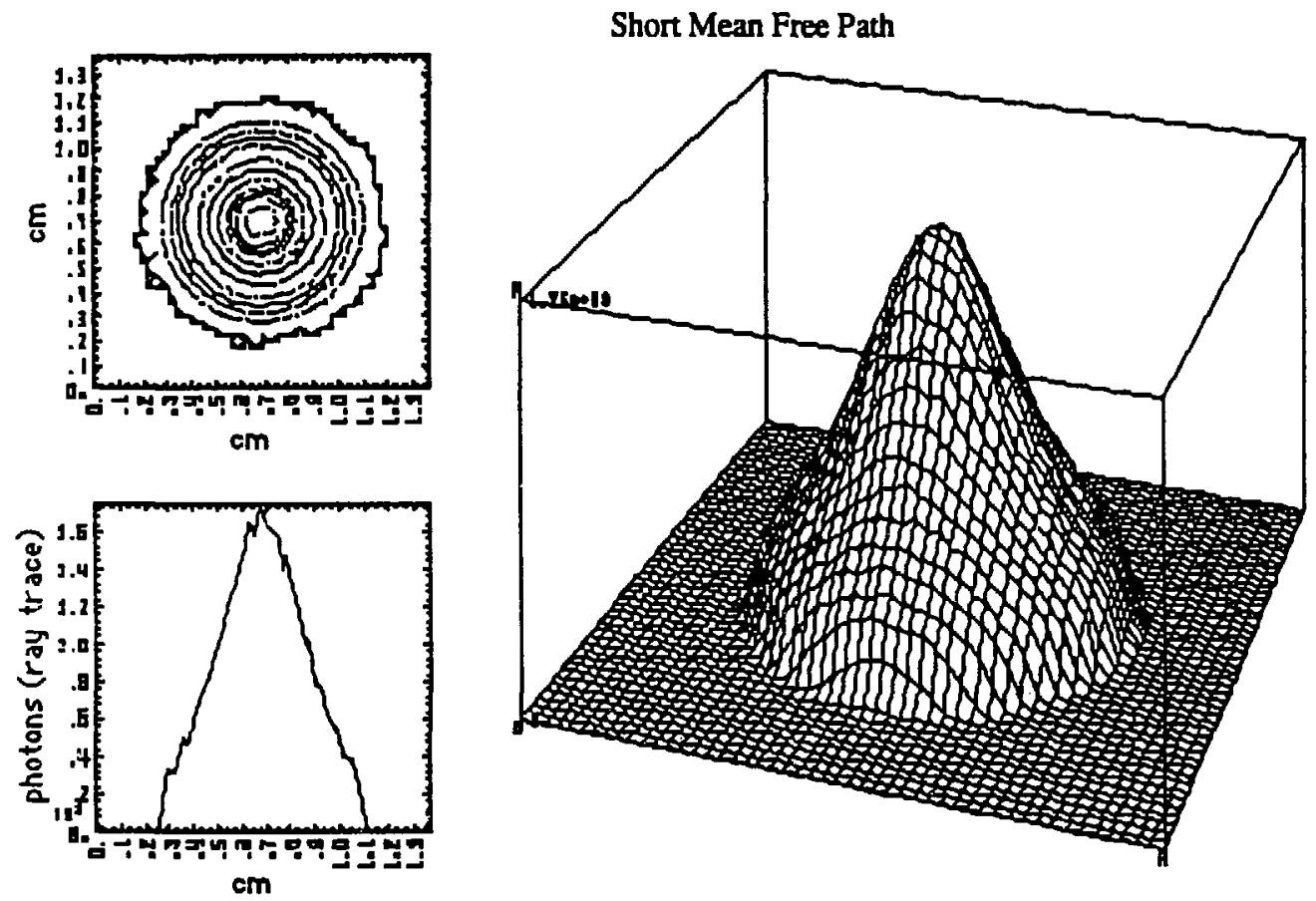

Long Mean Free Path
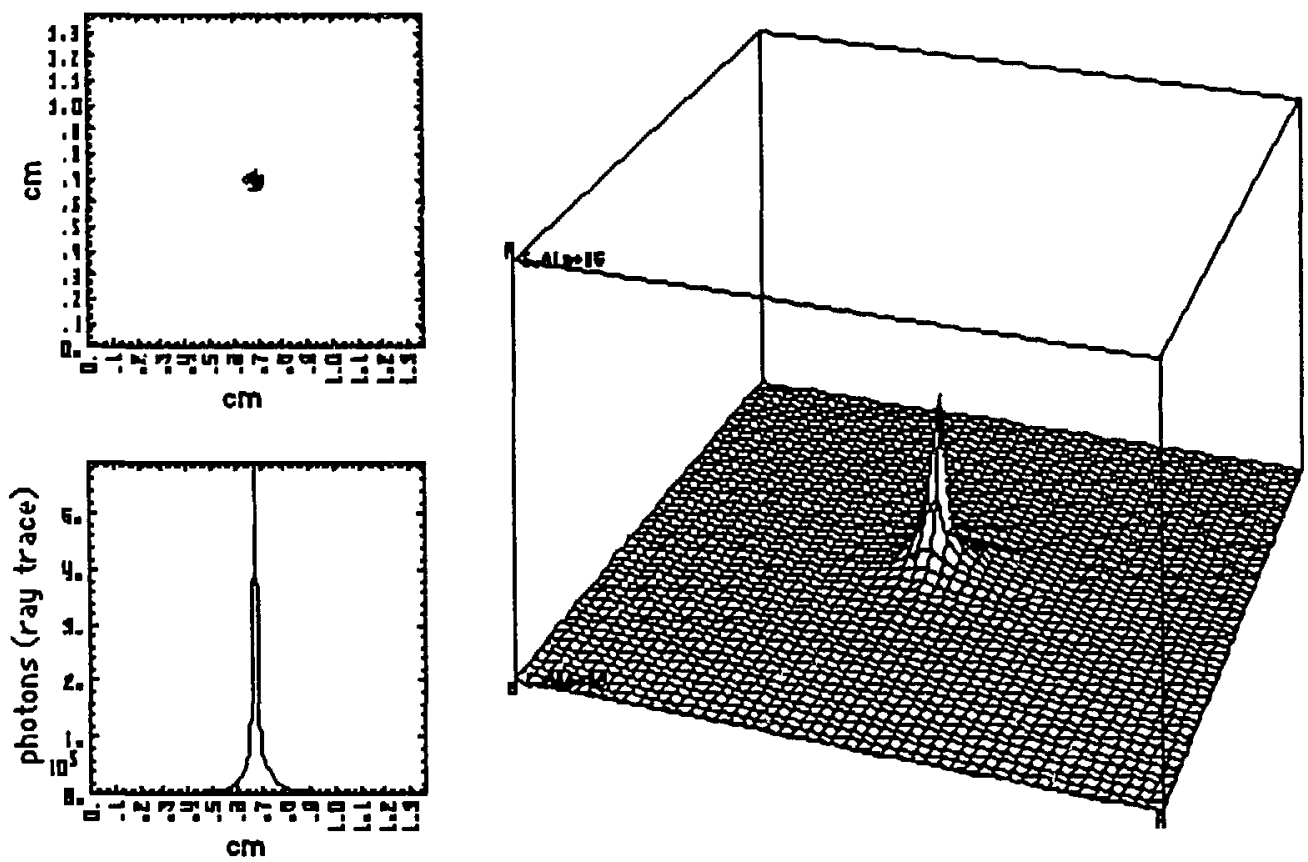

Figure IV.D.3. $I(r)$ in short and long absorption mean free path limits. 
where $I\left(r^{\prime}\right)$ is given by equation IV.D.3. The result of this procedure for .5 and $3 \mathrm{~mm}$ thicknesses of CsI are shown in figure IV.D.4. The dips occur at energies that correspond to the $\mathrm{K}$ absorption edges of Cs and I, where there is a jump in $\mu$. If $d_{\text {half }}$ is plotted as a function of $b_{\max }$ divided by $\lambda$, the two curves lie on top of each other (see figure IV.D.5). This is because the shape of $I(r)$ only depends on the number of $\mathrm{x}$-ray mean free paths across the crystal.

\section{E. Compton Scattering}

Up to this point only the photoelectric process has been considered, but $\mathrm{x}$ rays can also interact with matter via Compton scattering and pair production. At energies above $200 \mathrm{keV}$, Compton scattering begins to become important in CsI. Figure IV.E.1 shows how the reciprocal mean free path for each interaction and their sum varies with photon energy. The probability of one or the other of the two processes occurring as a function of energy is also shown. At about $300 \mathrm{keV}$ they are equally probable. Thus there is a question of how the spatial resolution will be affected.

In the Compton effect, a photon collides with an electron that is either free or quite loosely bound in an atom. During the collision, a new photon, with less energy than the one incident, is produced and scatters at an angle which depends on the amount of energy transferred to the electron. The energy of the recoil electron, $E_{c}$ is given by

$$
E_{c}=E_{\gamma}\left[1-\frac{1}{1+E_{\gamma}(1-\cos \theta)}\right]
$$




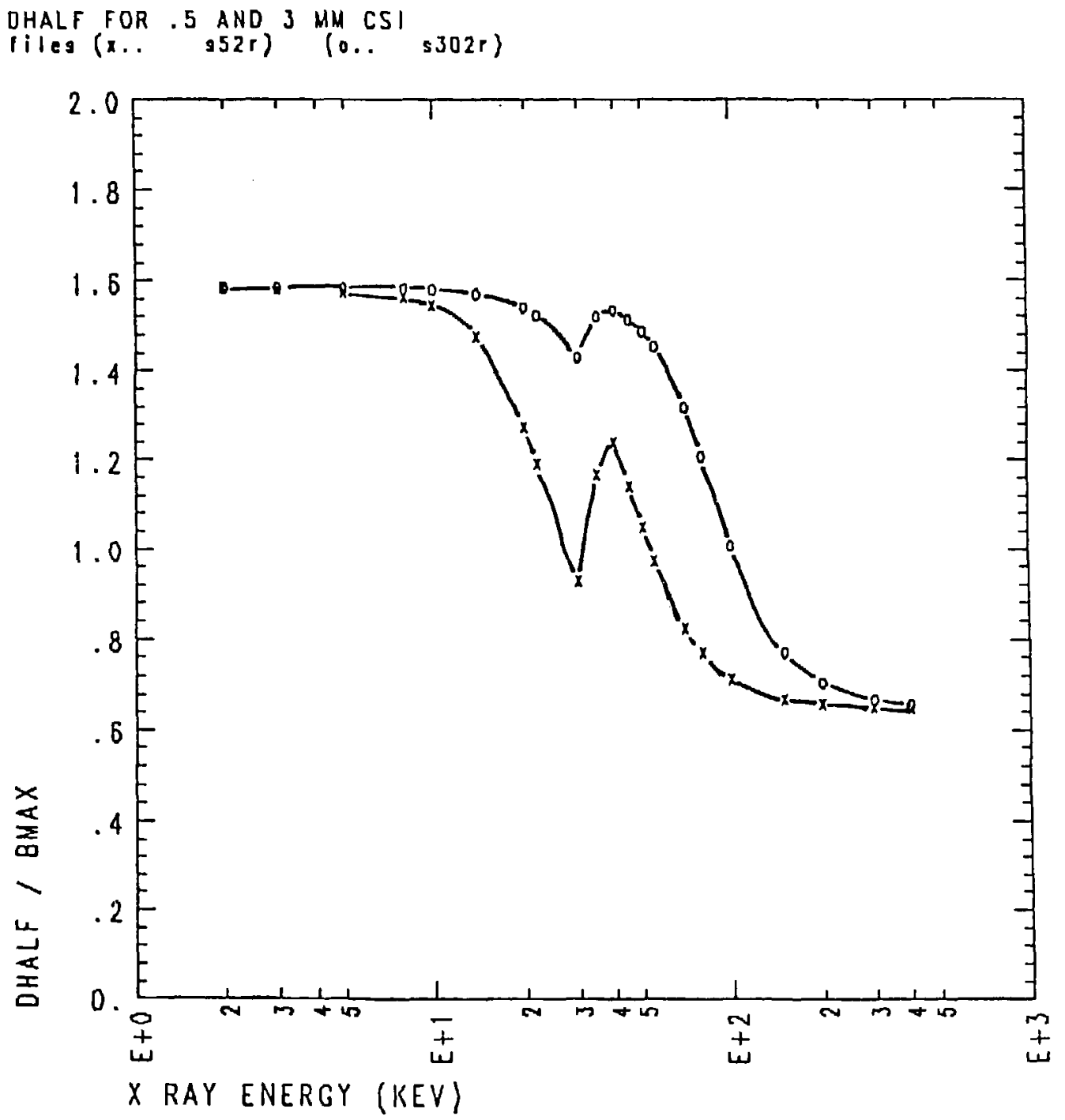

Figure IV.D.4. Variation of spot size with $x$-ray energy-points marked with $x(0)$ correspond to .5 (3) $\mathrm{mm}$ thickness of Csl. 
DHALF/BHAX VERSUS BHAX/LAMBDA

files (0.. 9302I) (X.. 5521)

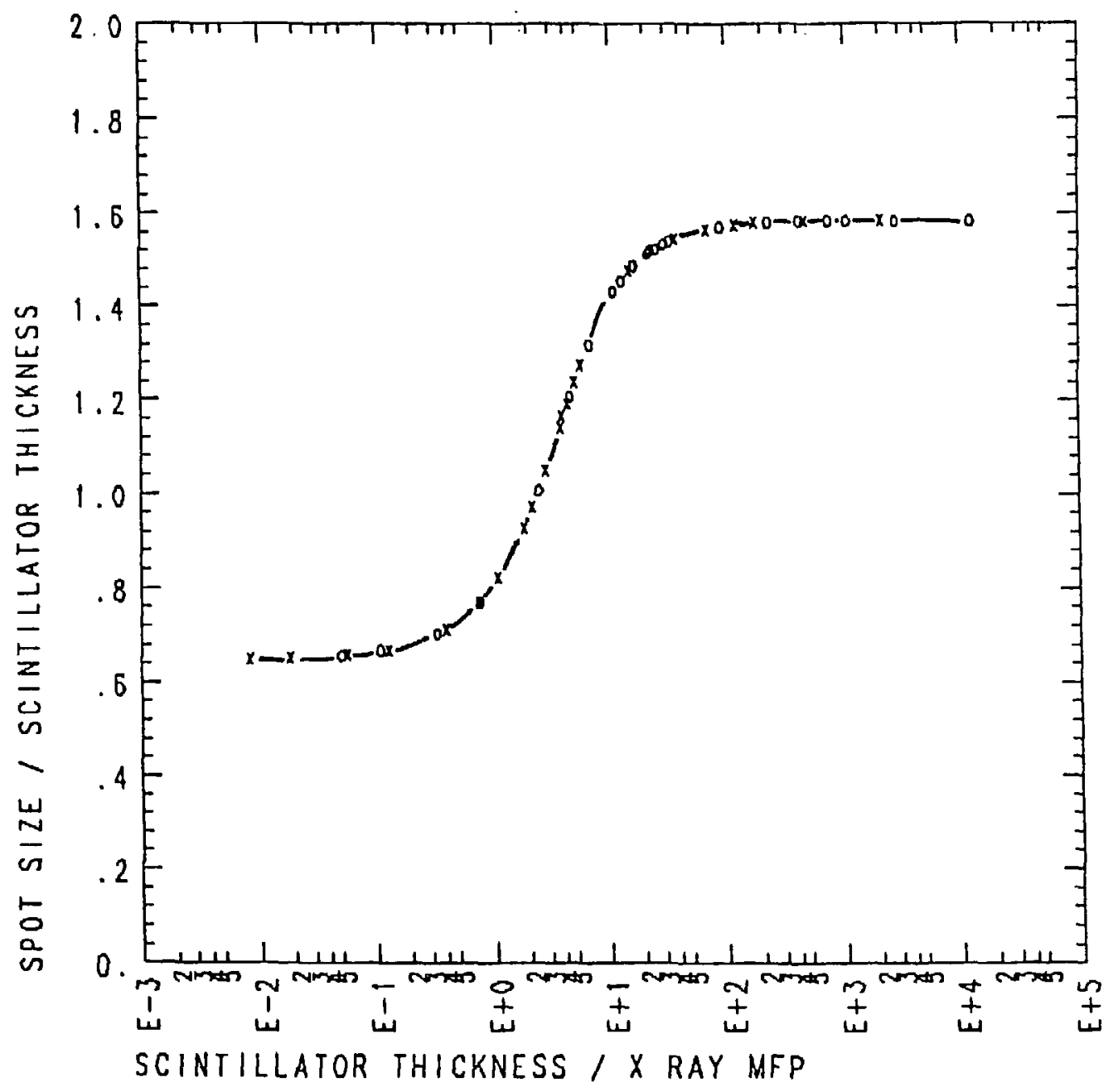

Figure IV.D.5. Variation of spot size with $x$-ray absorption mean free path. Points marked with $x(0)$ correspond to .5 (3) $\mathrm{mm}$ thickness of Csl. 

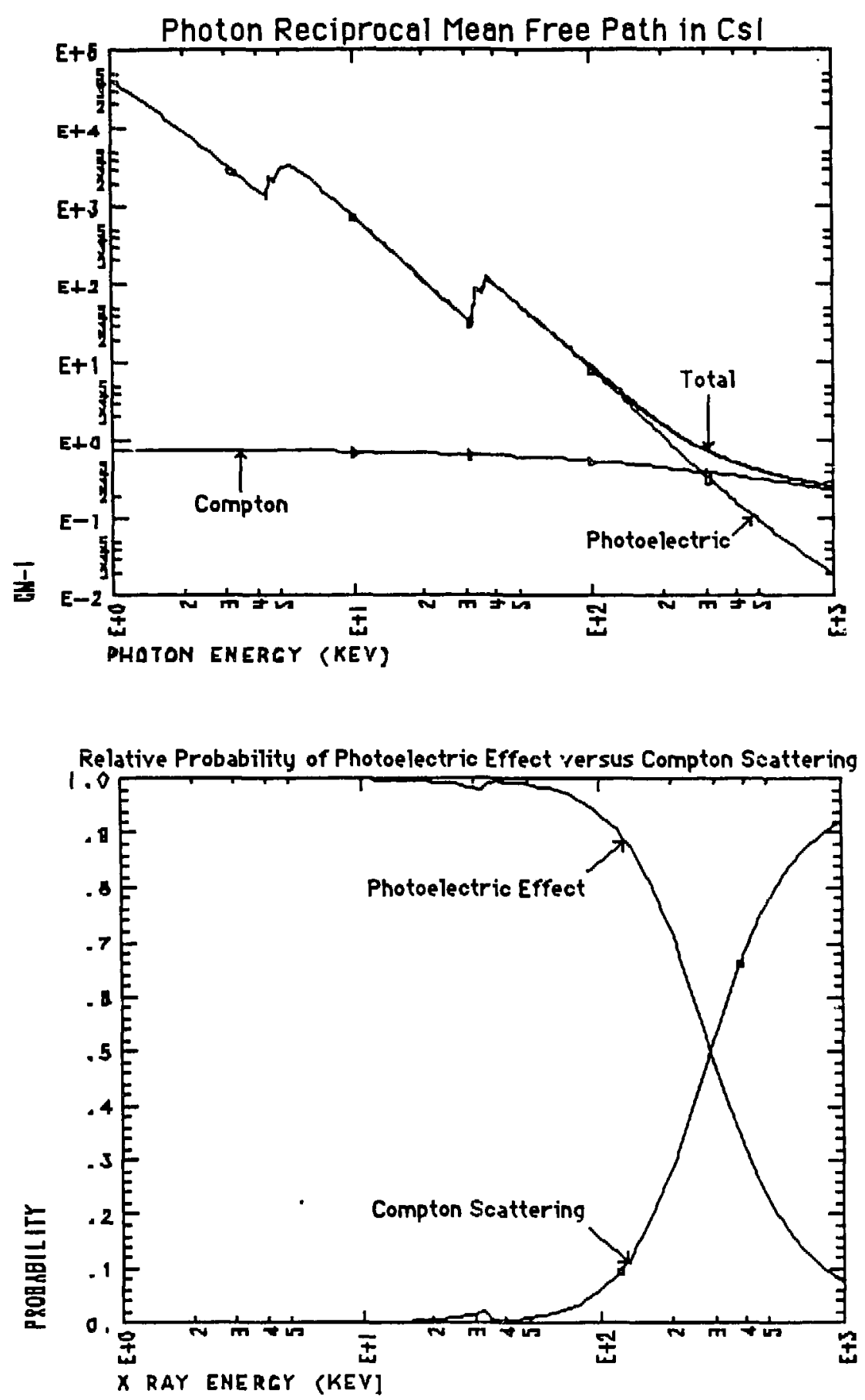

Figure IV.E.1. Photon interaction processes in CsI. 
where $E_{\gamma}$ is the x-ray energy, $\theta$ is the scattering angle, and both the $\mathrm{x}$ ray and electron energies are in units of the electron rest mass. ${ }^{1}$ When a photoelectric interaction occurs, all of the photon energy is deposited in the absorber. The process of Compton scattering is complicated because typically only a fraction of the $\mathrm{x}$ ray energy is deposited, while a scattered $x$ ray is produced and continues on with a new trajectory.

Because the process involves multiple interactions, its effect was evaluated with a Monte Carlo ray-tracing code. The response of a $3 \mathrm{~mm}$ thick CsI crystal to $50,100,200,300,450$, and $700 \mathrm{keV}$ x-rays was modeled. The area on the fiber optic was broken up into ten thousand 137 by 137 micron squares, corresponding to the microdensitometer aperture size used to digitize the data from the experiment (for comparison a typical pinhole-limited resolution element was about 1000 by 1000 microns). Because the photon energies were high (and the mean free paths long), the intensity profiles were similar to the one depicted at the bottom of figure IV.D.3.

One would expect the Compton scattering to produce a less peaked intensity profile, and this is indeed the case, although the effect is less than 1 part in 10 . To facilitate a quantitative comparison between the intensity profiles with and without scattering, the two-dimensional intensity data was averaged in one dimension (say $x$ ), and then normalized. Shown in figure IV.E. 2 is the difference between the averaged intensity data measured without Compton scattering to that measured with it. Because the averaged intensities have been normalized to the same value, the difference is due 
solely to variations in the shape of the intensity pattern. Because the scattering produces a slightly broader intensity pattern, the difference is positive near the center of the image and negative in the wings. The variations between the two simulations at $50 \mathrm{keV}$ (solid line) is statistical, but there are definite changes in shape at 100 (dotted) and $300 \mathrm{keV}$ (dashed). Plotted in figure IV.E. 3 is the average of the absolute value of the difference versus $x$ ray energy. This indicates that the effect of Compton scattering on the shape of the intensity pattern is most pronounced at energies where the Compton and photoelectric cross-sections are equal-for CsI this is at about $300 \mathrm{keV}$. The drop off in the effect at higher energies is due to the fact that, although the Compton effect is the dominant absorption mechanism, the $\mathrm{x}$ ray mean free path has become long compared to the crystal thickness, and multiple interactions are therefore less likely.

The effects of Compton scattering on resolution are small (at most $10 \%)$, and are most important at energies where the Compton and photoelectric cross-sections are comparable. ${ }^{2}$ The Compton energy absorption cross-section peaks at about $500 \mathrm{keV}$, which indicates that the change in the intensity spot shape is due to a scattering event followed by photoelectric absorption. Even though the effects of scattering on the intensity spot size are not first order, they are included in all subsequent calculations for completeness.

\section{F. Summary}

Up to this point, pinhole transmission and scintillator absorption have 
Differenca in Averaged Intenoltias

Fith and without Compton Scattering In the Solntillator

Difference Divded by Peak Intensity veruua

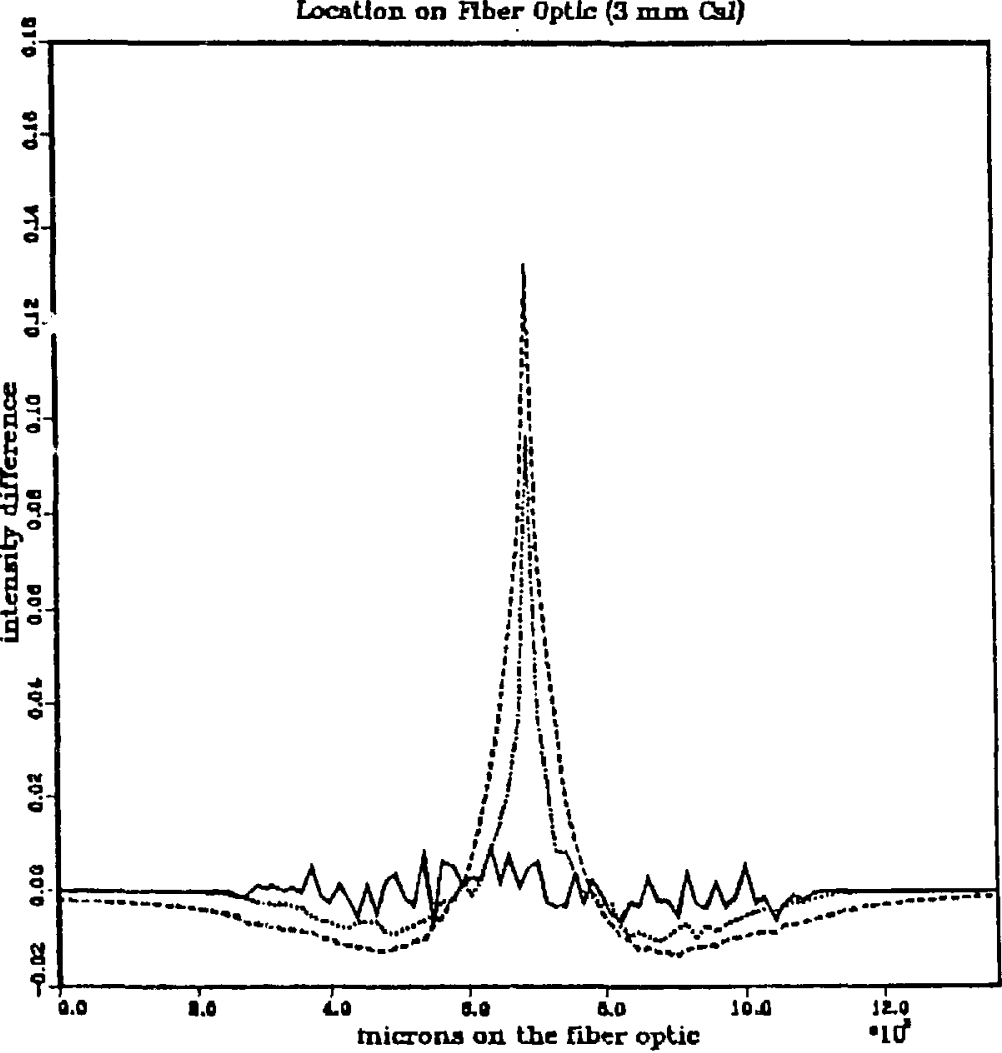

Figure IV.E.2. The three energies shown are: $50 \mathrm{keV}$ (solid), $100 \mathrm{keV}$ (dotted) and $300 \mathrm{keV}$ (dashed). 
Effects of Complon Scattering

on the Shape of the Intensity Spot

Produced by Different Energy X Rays

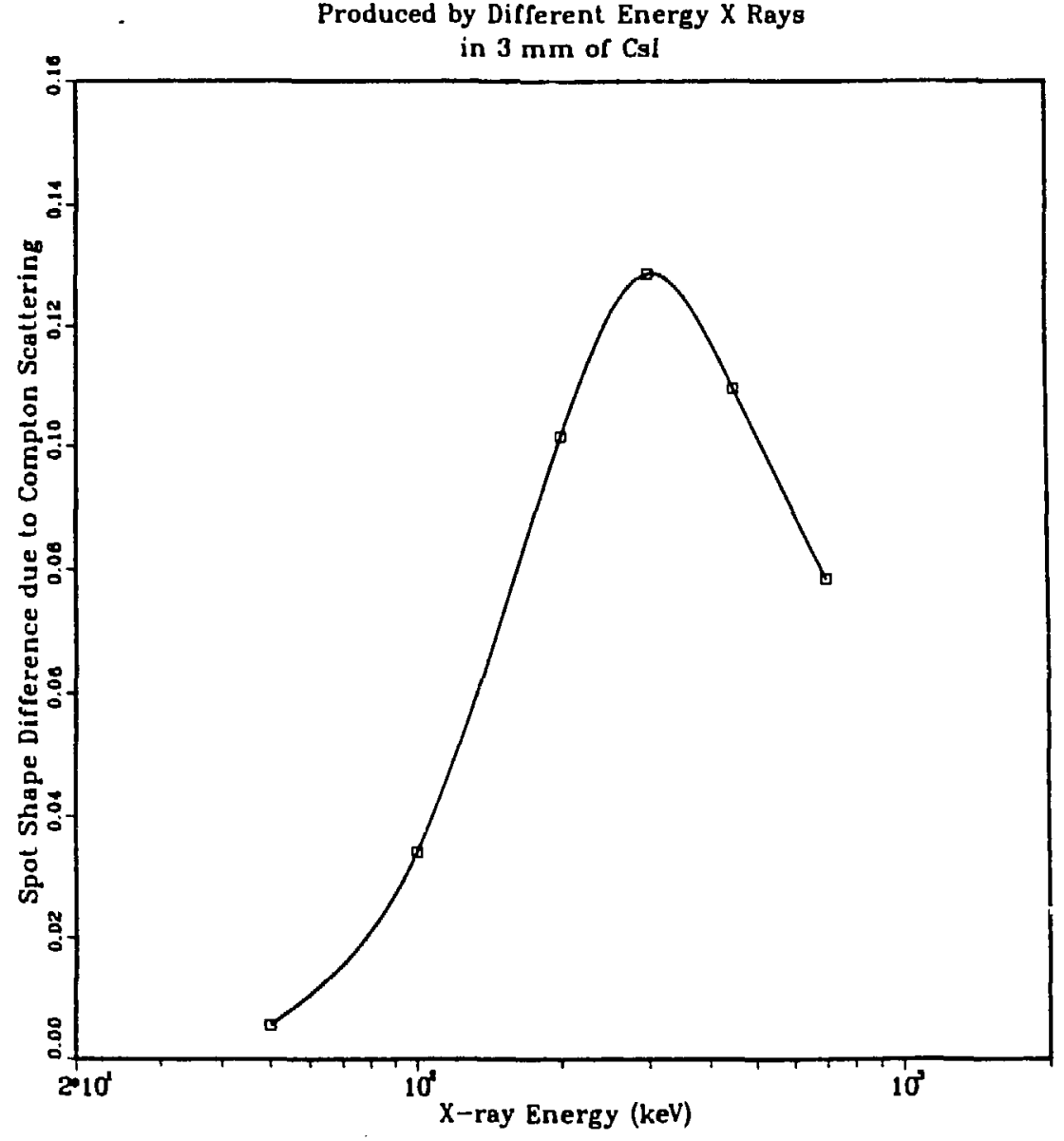

Figure IV.E.3. Relative intensity (spot shape) difference due to Compton scattering as a function of $\mathrm{x}$-ray energy. 
been discussed separately, but it is the convolution of the two that determines the resolution that can be achieved. To evaluate this, ray-trace simulations were done for $\mathrm{x}$-ray sources which had power spectra that fell off exponentially with energy

$$
\frac{d P}{d E} \propto \exp \left(-\frac{E}{T_{e}}\right)
$$

Values for $T_{e}$ of $5,20,100$, and $400 \mathrm{keV}$ were used. The responses of .5 and $3 \mathrm{~mm}$ thick CsI scintillators, with 1 and $2.8 \mathrm{~mm}$ diameter pinholes were simulated. in all cases, the incident power spectrum was attenuated by an 8 mil Al window.

A measure of the simulated spot size, $D_{s i m}$, was determined in the following way. First an average was done over the image in one orthogonal coordinate (call it $x$ ), and then the running integral, as a function of the other orthogonal coordinate $(y)$, was calculated. For a point source located at infinity, a circular pinhole, radius $r_{0}$, would produce an intensity in the $x-y$ image plane given by

$$
I(x, y)= \begin{cases}I_{0}, & \text { if } \sqrt{x^{2}+y^{2}} \leq r_{0} \\ 0, & \text { otherwise. }\end{cases}
$$

and the running integral, $I_{\text {run }}$, would therefore be

$$
\begin{aligned}
I_{r u n}(y) & =\int_{-\infty}^{y} d y^{\prime} \int_{-\infty}^{\infty} d x^{\prime} I\left(x^{\prime}, y^{\prime}\right) / I_{0} \\
& =\frac{1}{2}+\frac{1}{\pi}\left[\frac{y}{r_{0}} \sqrt{1-\left(\frac{y}{r_{0}}\right)^{2}}+\arcsin \frac{y}{r_{0}}\right] .
\end{aligned}
$$

If $D_{\text {sim }}$ is defined as the interval between two values of $y$ which satisfy the relations

$$
I_{\text {run }}\left(y_{.25}\right)=.25
$$


and

$$
I_{\text {run }}\left(y_{.75}\right)=.75
$$

then for the circular pinhole described above,

$$
D_{s i m}=y_{.75}-y_{.25}=.810 r_{0}
$$

So if $D_{s i m}$ calculated from the simulation result is larger than $.810 r_{0}$, the resolution of the system wili be affected by the crystal thickness, the finite transmission of the pinhole, or both.

Shown in figure IV.F.1 are the results of the simulations. The different curves correspond to the different pinhole/scintillator combinations: $1 \mathrm{~mm}$ diameter pinhole and $.5 \mathrm{~mm}$ scintillator thickness (dashed), $2.8 \mathrm{~mm}$ diameter and $.5 \mathrm{~mm}$ thickness (solid), $1 \mathrm{~mm}$ diameter and $3 \mathrm{~mm}$ thickness (chain), and $2.8 \mathrm{~mm}$ diameter and $3 \mathrm{~mm}$ thickness (dotted). The two curves for the $.5 \mathrm{~mm}$ scintillator thickness show little variation with $T_{e}$, and $D_{s i m}$ is determined, to within the error, by the pinhole diameter. The curves corresponding to the $3 \mathrm{~mm}$ thickness to show variation with $T_{e} . I(y)$ for the $2.8 \mathrm{~mm}$ pinhole diameter and different values of $T_{e}$ is plotted in figure IV.F.2. The corresponding plots for the $1 \mathrm{~mm}$ pinhole are shown in figure IV.F.3. The solid curve corresponds to a $T_{e}$ of $5 \mathrm{keV}$, the dotted 20 , the dashed 100, and the chain 400 . For the $2.8 \mathrm{~mm}$ pinhole, when $T_{e}$ is $400 \mathrm{keV}$, the intensity profile has sharpened enough to become pinhole limited, but at the lower values, $D_{\text {sim }}$ is independent of the pinhole diameter, and is thus limited by the crystal thickness. The fact that $D_{\text {sim }}$ is larger for the 1 than 
the $2.8 \mathrm{~mm}$ pinhole when $T_{e}=400 \mathrm{keV}$ can be attributed to a loss in pinhole sharpness due to increased transmission.

All of these results are consistent with the calculations done in earlier sections. We found that in the short mean free path limit, $d_{\text {half }} \rightarrow 1.55 b_{\text {max }}$. So in that limit $d_{h a l f}$ is .775 for the thin and $4.65 \mathrm{~mm}$ for the thick crystal. Because .775 is less than 1 , the $D_{s i m}$ for the thin crystal was always pinhole limited. And since $4.65>2.8, D_{s i m}$ was limited by the crystal thickness for the $3 \mathrm{~mm}$ scintillator when $T_{e}$ was small. In the long mean free path limit, $d_{\text {half }} \rightarrow .638 b_{\max }$ or $.191 \mathrm{~mm}$ for the thick crystal. Thus it was possible for the spot size to become pinhole limited when $T_{e}$ became large enough.

The simulations provide guidance as to what combinations are appropriate for use on the experiment. For example, if increasing the detected signal is important, the $2.8 \mathrm{~mm}$ pinhole should be used with the $3 \mathrm{~mm}$ crystal, rather than the $1 \mathrm{~mm}$ pinhole. This is because the resolution obtained with the two pinhole diameters is the same, at electron temperatures below $400 \mathrm{keV}$. (In both cases the resolution is limited by the crystal thickness.) Also, for looking at a source with a $T_{e}$ of about $400 \mathrm{keV}$ or more, it would be better to use the $3 \mathrm{~mm}$ instead of the $.5 \mathrm{~mm}$ crystal with the $2.8 \mathrm{~mm}$ pinhole. This is because the sigral absorbed would be higher for the same spatial resolution.

In this section the factors which determine the system resolution have been discussed. It has been shown that the resolution can be influenced by the pinhole diameter and finite transmission, as well as the scintillator 


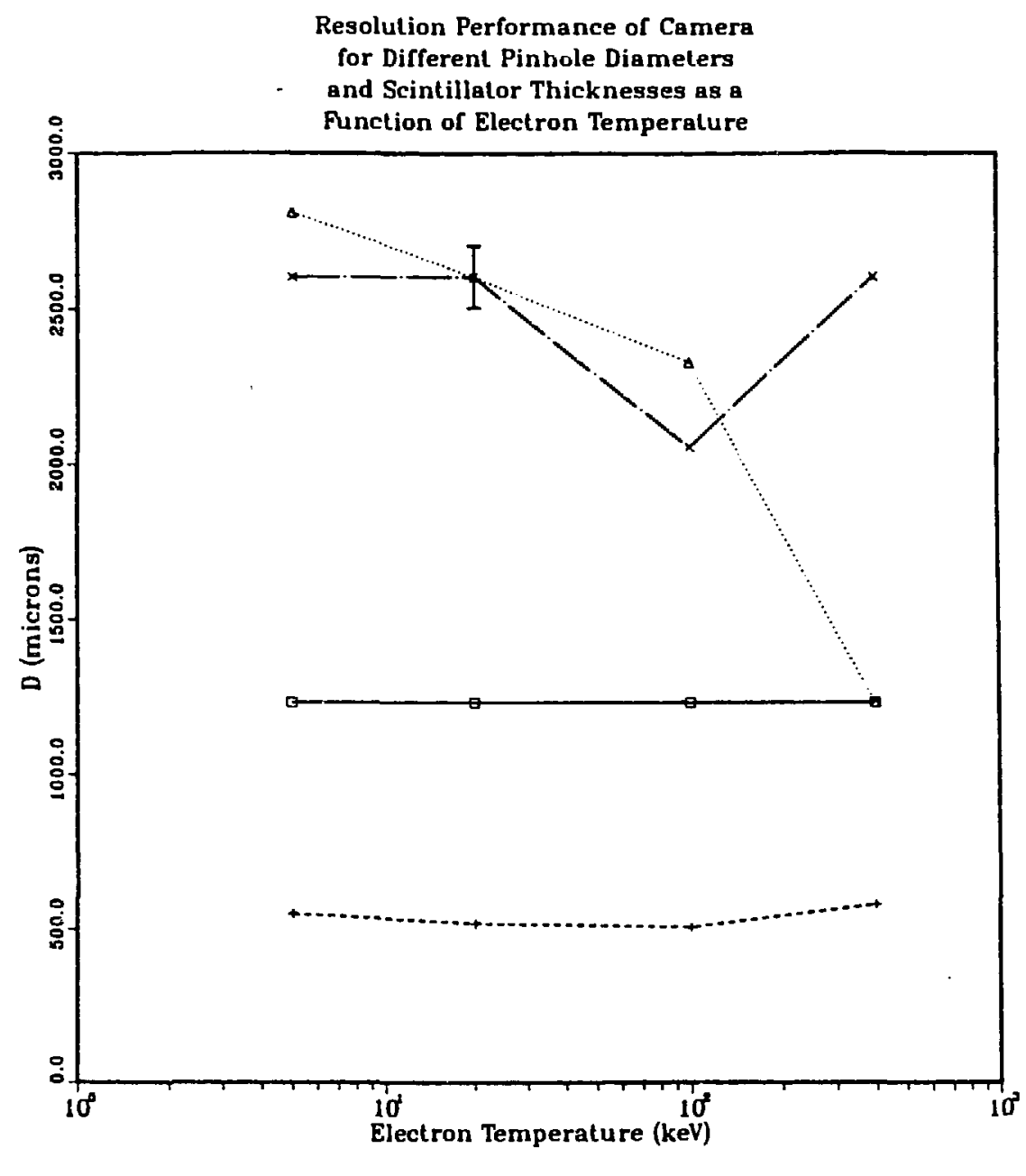

Figure IV.F.1. The four curves correspond to different pinhole/scintillator combinations: $1 \mathrm{~mm}$ pinhole diameter ano $.5 \mathrm{~mm}$ scintillator thickness (dashed), $2.8 \mathrm{~mm}$ diameter and $.5 \mathrm{~mm}$ thickness (solid), $1 \mathrm{~mm}$ diameter and $3 \mathrm{~mm}$ thickness (chain), and $2.8 \mathrm{~mm}$ diameter and $3 \mathrm{~mm}$ thickness (dotted). 
Comparison of Spot Sizes Produced by a $2.8 \mathrm{~mm}$ Diameter Pinhole and a

$3 \mathrm{~mm}$ Thick Csl Scintillator for

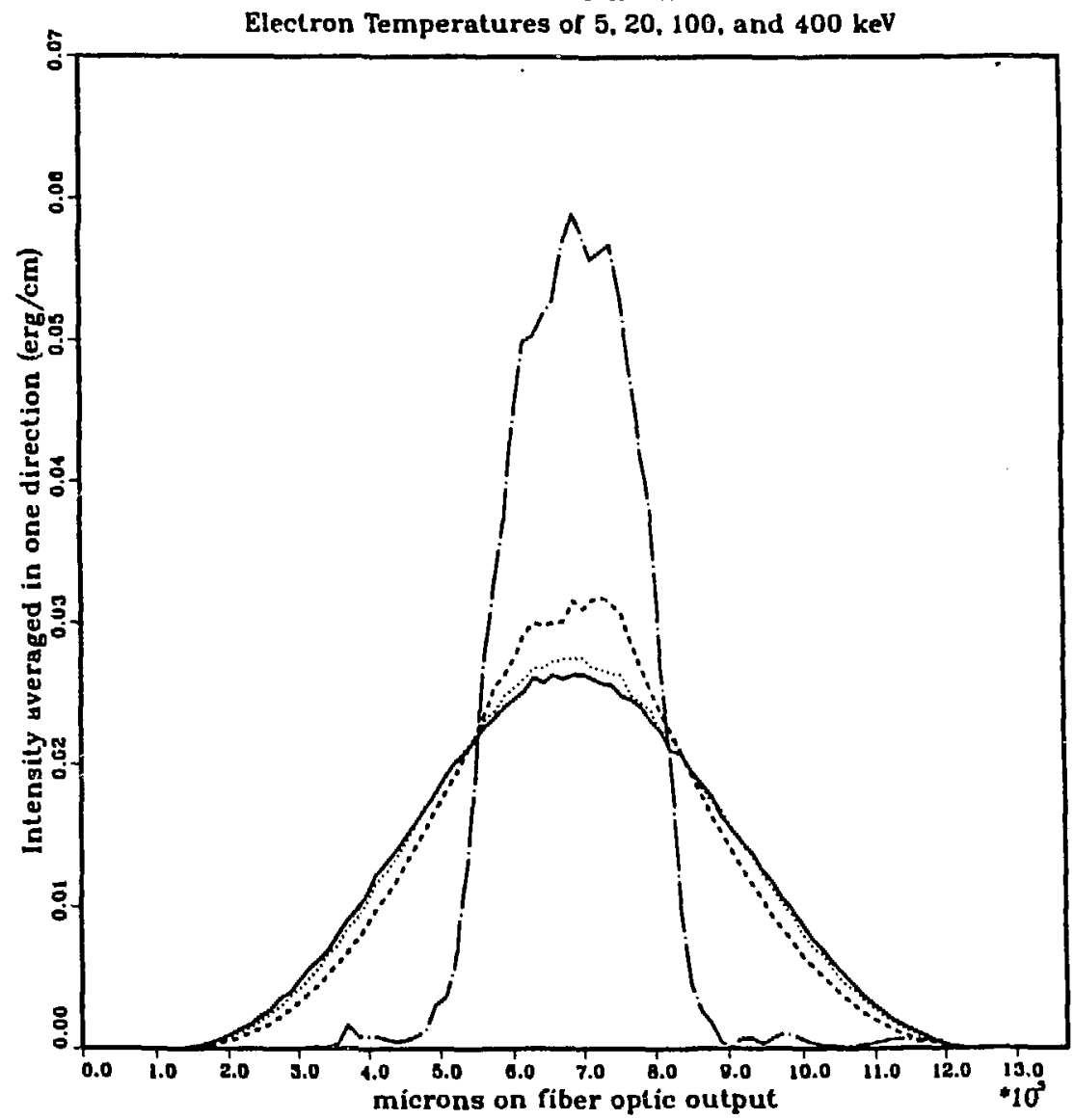

Figure IV.F.2. The four curves correspond to electron temperatures of 5 (solid), 20 (dotted), 100 (dashed), and $400 \mathrm{keV}$ (chain). 
Comperison of Spot Sizes Produced by a $1 \mathrm{~mm}$ Diameter Pinhole and a

$3 \mathrm{~mm}$ Thick Csl Scintillator for

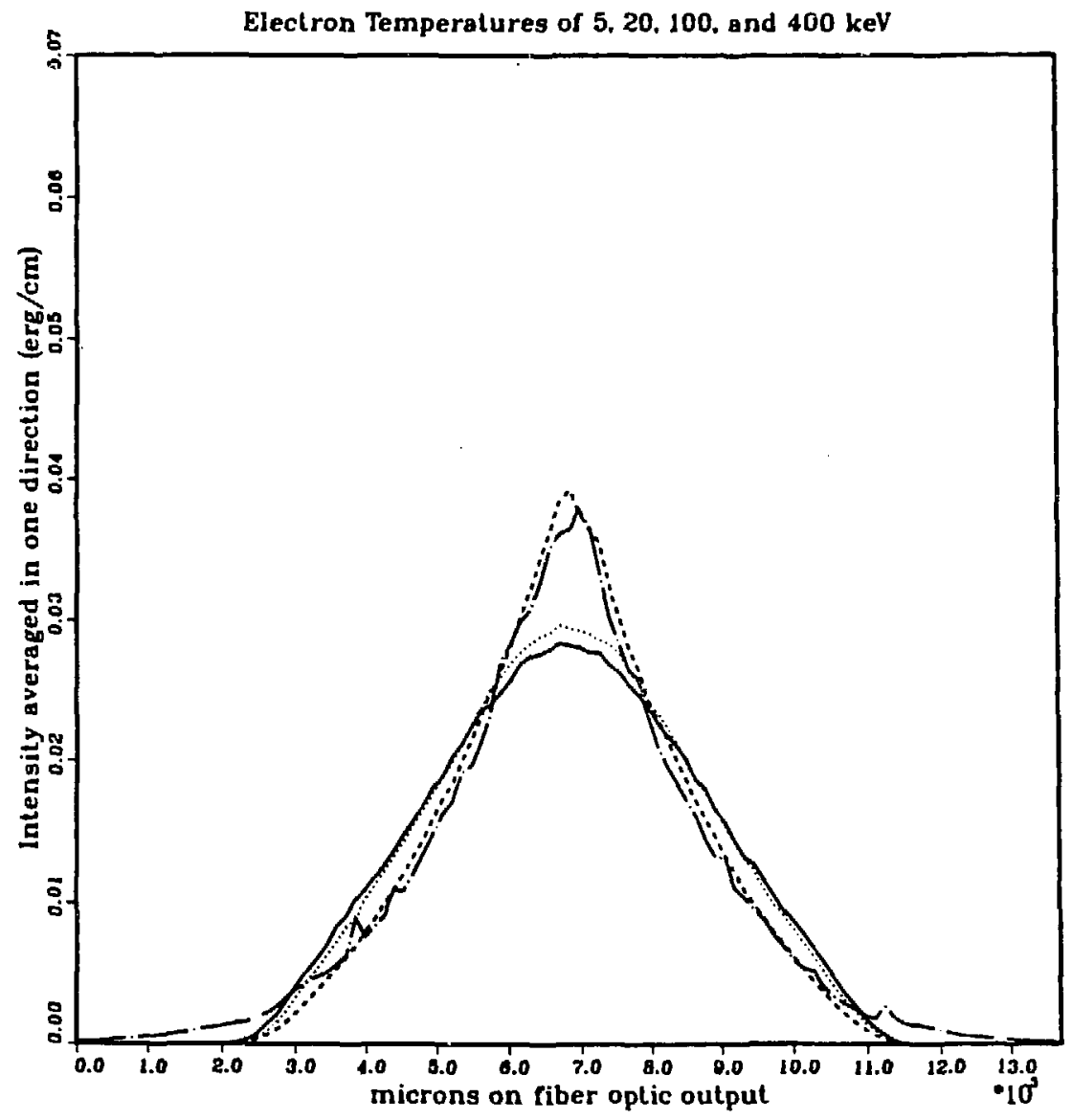

Figure IV.F.3. The four curves correspond to electron temperatures of 5 (solid), 20 (dotted), 100 (dashed), and $400 \mathrm{keV}$ (chain). 
thickness. It is also clear that the pinhole/scintillator combination can be chosen to optimize resolution and signal, and that this choice depends on the distribution of $x$-ray energies emitted by the source. 


\section{Chapter V}

\section{Imag: Analysis}

\section{A. Introduction}

In the previous chapter the factors which influence camera resolution, pinhole size, pinhole transmission, and scintillator thickness, were discussed. This information guided the design and implementation of the high energy $\mathrm{x}$-ray camera. Once an image had been obtained, effects due to the pinhole geometry and plasma shape were removed before axial and radial profiles could be determined from the measured intensities.

The analysis of the image is done in three steps. First, collimation associated with the pinhole, and plasma ellipticity due to the shape of the confining magnetic field must be considered. After these two effects have been compensated for the data can be Abel inverted to obtain radial and axial x-ray emissivity profiles.

\section{B. Pinhole Collimation}

The fact that the pinhole must have a finite thickness (in order to maintain image clarity at high $\mathrm{x}$-ray energies), means that it will behave like a collimator. That is, the effective solid angle subtended by the pinhole at the source, will depend on the location of the source. The pinholes used for the imaging system consists of a cylindrical hole, that is counterbored from both sides to form two cones whose axes and tips coincide. (See figure IV.C.1) 
There are two different effects: (1) foreshortening of the aperture in the absence of collimation and (2) eclipsing of the two apertures. For the pinholes used on TMX-Upgrade the first effect is not important, but the second one is. Both are a function of the angle between the pinhole axis and the line that connects the source point and the center of the pinhole.

The reduction of effective pinhole area as the result of foreshortening is given by

$$
A_{e f f}=A \cos (\phi)=A / \sqrt{1+\left(\frac{R}{L}\right)^{2}}
$$

where

$$
\phi=\arctan \left(\frac{R}{L}\right)
$$

where $L$ is the distance from the pinhole to the source plane, and $R$ is the distance from the pinhole axis to a source point in that plane. The area of the pinhole goes to zero as $\phi$ goes to $\frac{\pi}{2}$, which is equivalent to $R$ becoming much greater than $L$. To see how important this effect is, consider an example pertinent to the geometry of the TMX-U plug system. For the values

$$
\begin{array}{r}
R=35 \mathrm{~cm} \\
L=262 \mathrm{~cm}
\end{array}
$$

the correction to the aperture area is

$$
\frac{A-A_{\text {eff }}}{A} \approx .009
$$

or less than one percent. This is a negligible effect in comparison with other experimental uncertainties. 
The fact that the pinhole acts as a collimator is an important effect. As the source point moves away from the pinhole axis, the openings at the ends of the cylindrical section move out of alignment, which reduces the effective area of the pinhole. This reduced area is given by

$$
A_{\text {red }}=A\left[1-\frac{2}{\pi}\left(\arcsin x-x \sqrt{1-x^{2}}\right)\right]
$$

where

$$
x=\frac{R l}{r L}
$$

and $r(l)$ is the radius (length divided by two) of the pinhole cylindrical section, as depicted in figure IV.C.1. Expressing $A_{\text {red }}$ as a power series in x, one has

$$
A_{\text {red }}=A\left[1-\frac{4}{\pi}\left(x-\frac{1}{6} x^{3}-\frac{1}{40} x^{5}-\frac{1}{112} x^{7}-\frac{1}{230.4} x^{9}-O\left(x^{11}\right)\right)\right] .
$$

The dependence of $A_{\text {red }}$ on $\mathrm{x}$ is shown in figure V.B.1. Both the complete form and the linear approximation (good for small $x$ ) are shown. Now, inserting the same values for $R$ and $L$ as above into equation (V.B.1), with

$$
\frac{r}{l}=.27670
$$

the area is reduced by

$$
\frac{A-A_{\text {red }}}{A} \approx .590
$$

This is a sizable correction. Fortunately, it is not difficult to make because it is constant for each line of sight.

An example of how the image is distorted by the coilimation is shown in figures V.B.2 and V.B.3. These were two ray-tracing simulations of images 
Collimalion Efrect

Holh the Exacl and Linear Approximation Are Shown

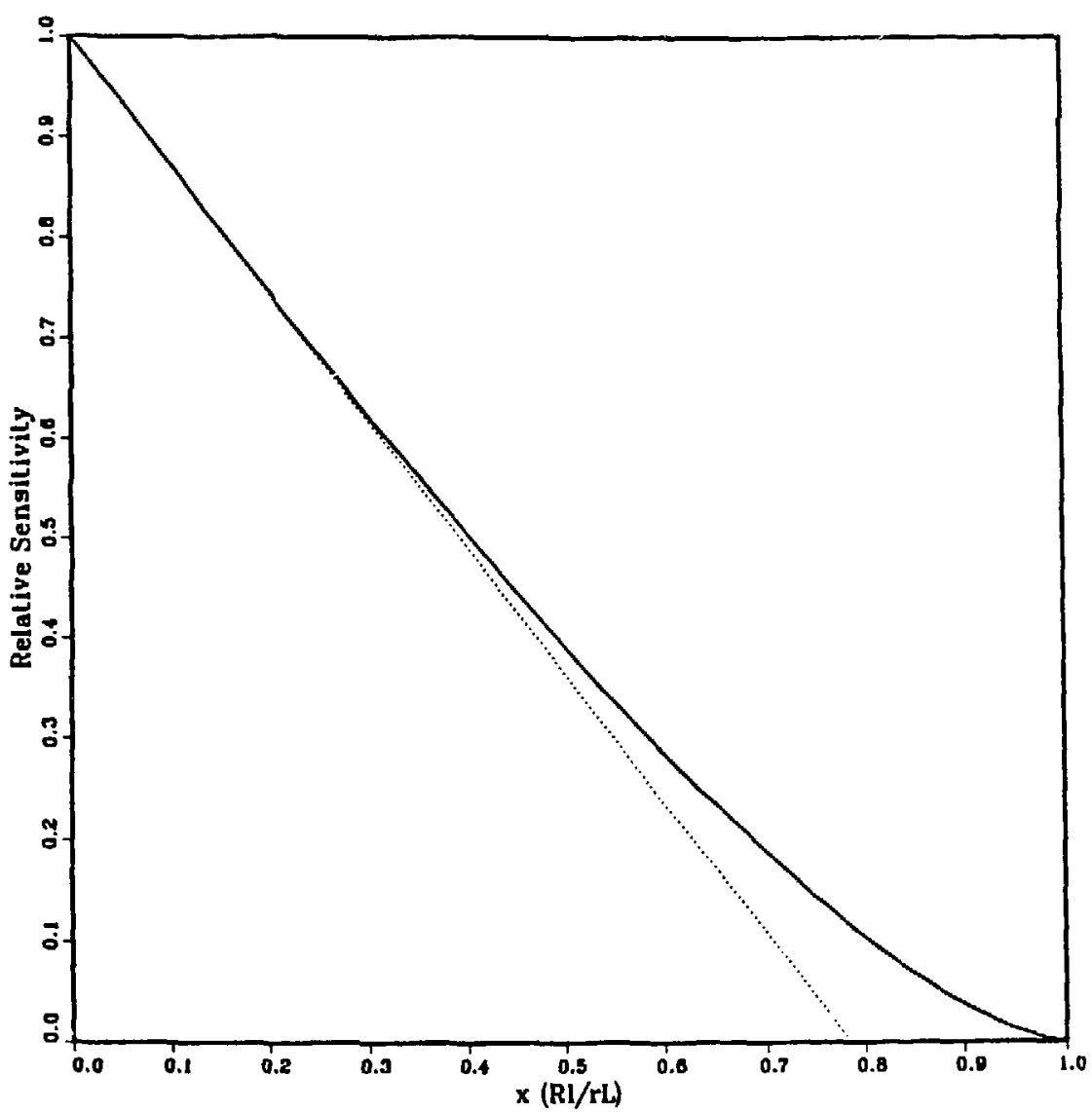

Figure V.B.1. The solid (dotted) curve corresponds to the exact result (linear approximation). 
produced by volume emission in the west plug. The axial variation of the intensity measured on axis is due to the fact that the plasma is fanning (the explanation for this fanning is given in the next section). The intensity measured for the run without collimation effects included is peaked where the plasma cross section is most elliptical, while the run that includes those effects is peaked near the axis of the pinhole.

\section{Plasma Ellipticity}

The ellipticity of the plasma cross-section varies throughout the end cells of TMX-Upgrade. This is due to the fact that the end cell magnetic fields have a twisted bow tie shape characteristic of a minimum $|\mathbf{B}|$ configuration. (See figure V.C.1.) The machine coordinate system of TMX-Upgrade is oriented so that the $x$-axis points south, the $y$-axis points up, and the $z$ axis points west. Axial locations are specified by their $z$ value, and radial locations by the perpendicular distance to the $z$-axis. This coordinate system is referred to throughout the remainder of this chapter. The surfaces of constant $|B|$ are circular at the magnetic midplane $(z=568)$, but fan in the $y$ direction for $z<568$, and in the $x$ direction for $z>568$. In figure V.C.2 the ellipticity of the plasma cross-section is shown for three axial locations- 530 , 552 , and $568 \mathrm{~cm}$. Before the data can be Abel inverted, this ellipticity must be removed. Fortunately there is a straightforward procedure to accomplish this.

The first step in doing this is to transform to a $\mathrm{f}_{\mathrm{l}}$ ame of reference in which the plasma is circular. If $a(b)$ is the $x(y)$ axis of the ellipse, the 


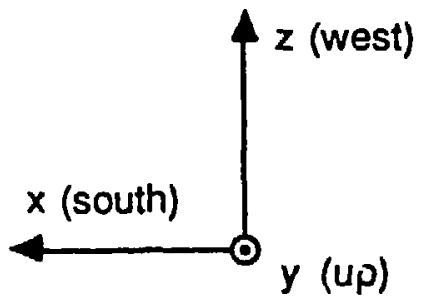

\section{No Colimation}
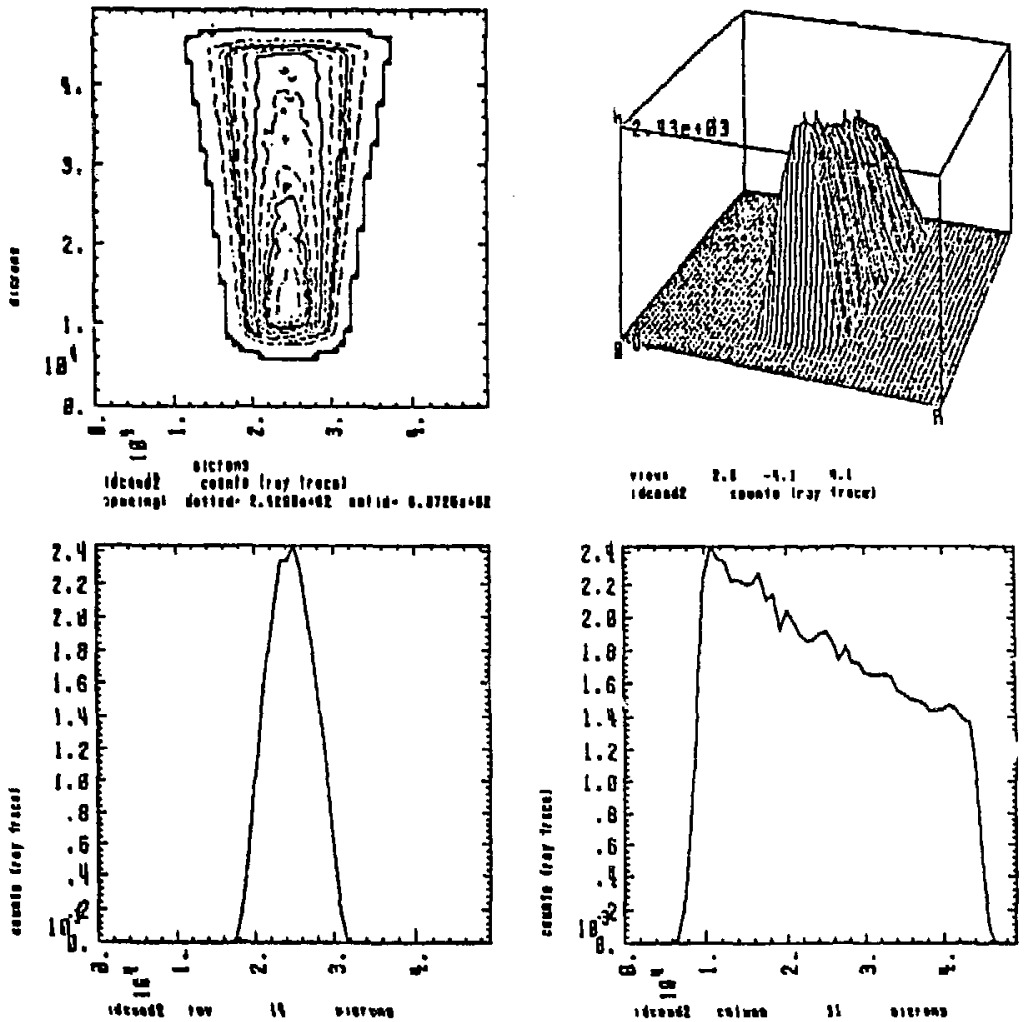

Figure V.B.2. Ray trace simulation of the detected image produced by a parabolic $x$-ray emissivity-no collimation effects. Shown are (clockwise from upper left): a (1) contour, (2) pseudo-3D, (3) radial lineout, and (4) axial lineout (each lineout passing through the location of maximum intensity). 


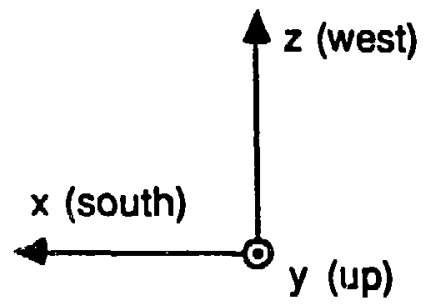

\section{With Collimation}
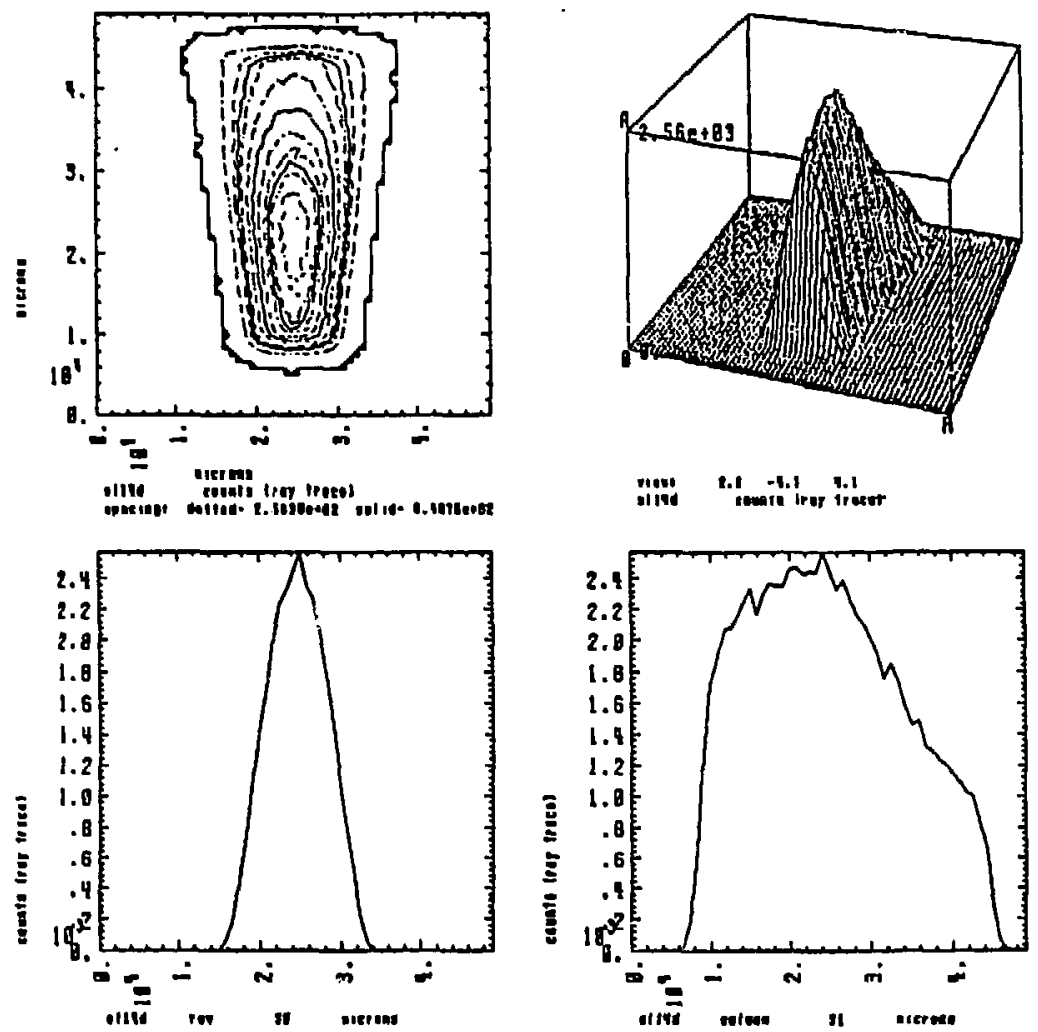

Figure V.B.3. Ray trace simulation of the detected image produced by a parabolic $x$-ray emissivity-with collimation effects. Shown are (clockwise from upper left): a (1) contour, (2) pseudo-3D,

(3) radial lineout, and (4) axial lineout (each lineout passing through the location of maximum intensity). 


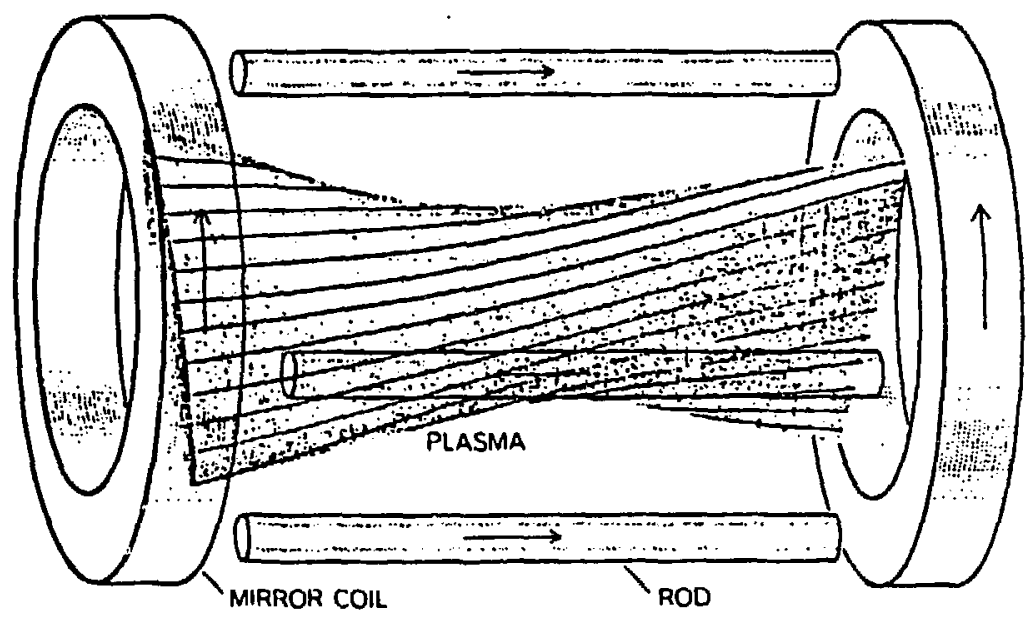

Figure V.C.1. Twisted bow tie shape characteristic of end plug plasma due to quadrupole fields. 
Variation in Plasma Elliplicity in HesL Plug

Approximeiions to the $|B|$ Surfaces are Shown

For $Z$ Values of 530,552 and $568 \mathrm{~cm}$.

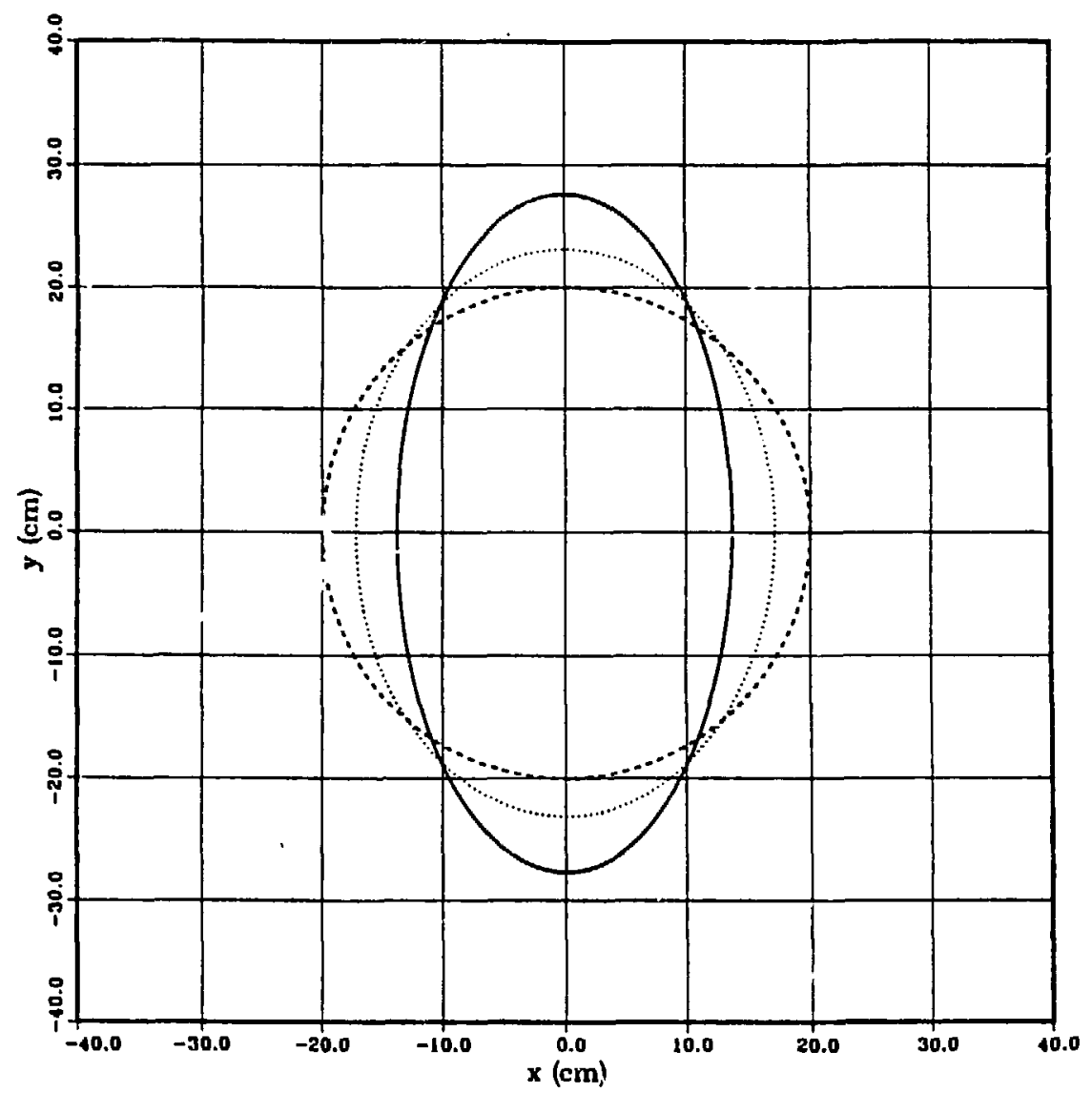

Figure V.C.2. The three axial locations shown are: 530 (solid), 552 (dotted), and $568 \mathrm{~cm}$ (dashed). 
transformation is

$$
\begin{aligned}
& x^{\prime}=\frac{x}{a} \\
& y^{\prime}=\frac{y}{b}
\end{aligned}
$$

See figure V.C.3.

For a pinhole camera, all the points on the image share the pinhole location as another point on their line of sight. The ratio of the length of integration in one frame to that in the other is

$$
\frac{l^{\prime}}{l}=\frac{\sqrt{\Delta x^{\prime 2}+\Delta y^{\prime 2}}}{\sqrt{\Delta x^{2}+\Delta y^{2}}}
$$

where

$$
\begin{aligned}
& \Delta x=x_{d}-x_{p} \\
& \Delta y=y_{d}-y_{p}
\end{aligned}
$$

and

$$
\begin{aligned}
& \Delta x^{\prime}=\frac{\Delta x}{a} \\
& \Delta y^{\prime}=\frac{\Delta y}{a}
\end{aligned}
$$

The pinhole is located at the point $\left(x_{p}, y_{p}\right)$, and the $\mathrm{x}$ rays are being detected at $\left(x_{d}, y_{d}\right)$. as shown in the previous figure. The distance of closest approach to the axis of syrnmetry (the z-axis in this case) in the new frame of reference, $u$, is given by

$$
u=\frac{\left|y^{\prime} \Delta x^{\prime}-x^{\prime} \Delta y^{\prime}\right|}{\sqrt{\Delta x^{\prime 2}+\Delta y^{\prime 2}}}
$$

where $\left(x^{\prime}, y^{\prime}\right)$ is any point on the line of sight determined by the two points $\left(x_{p}^{\prime}, y_{p}^{\prime}\right)$ and $\left(x_{d}^{\prime}, y_{d}^{\prime}\right)$, and the intensity as a function of $u$ is given by

$$
I^{\prime}(u)=I(u) \times\left(\frac{\sqrt{\Delta x^{\prime 2}+\Delta y^{\prime 2}}}{\sqrt{\Delta x^{2}+\Delta y^{2}}}\right)
$$



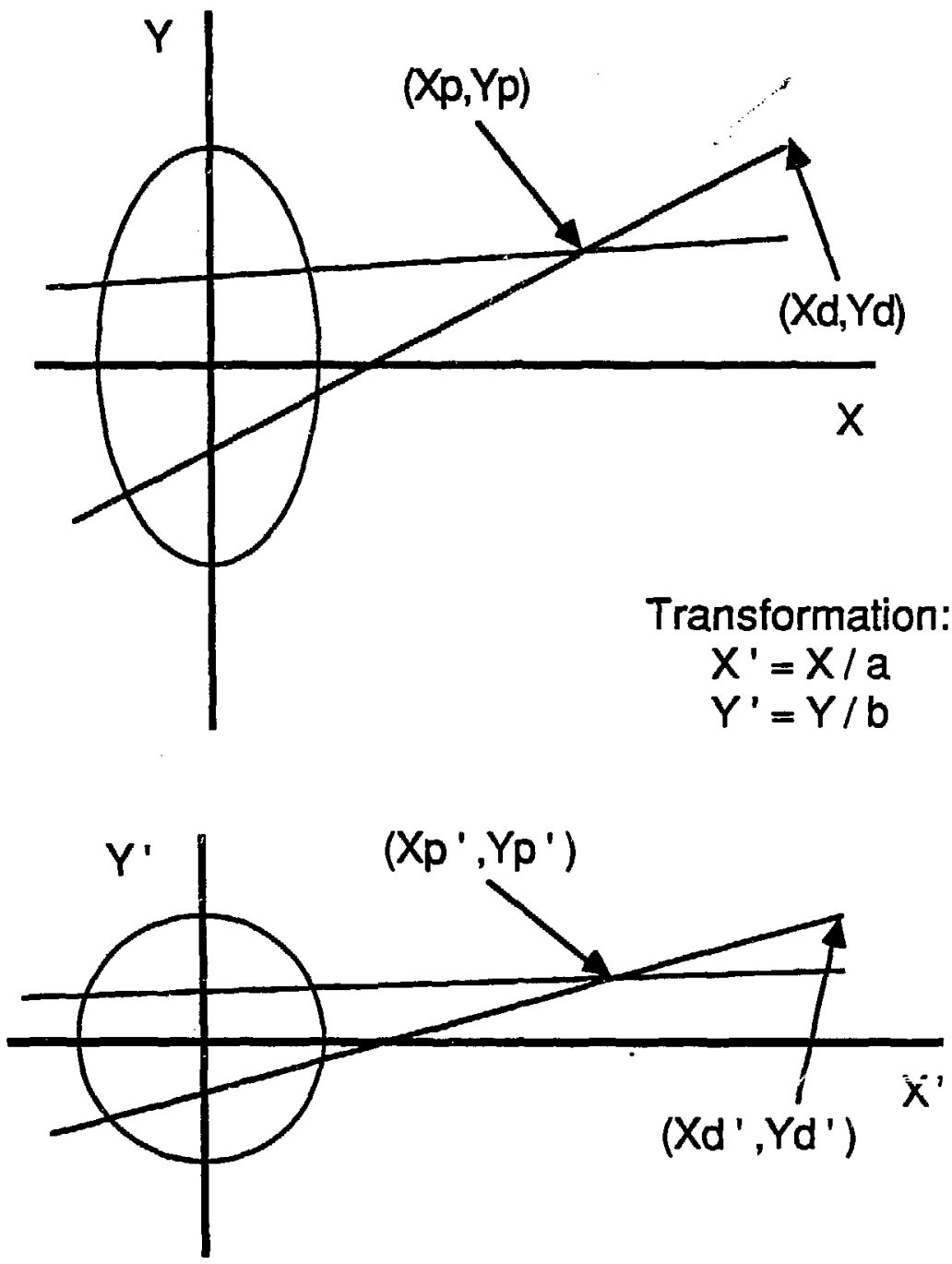

Figure V.C.3. Transformation to remove ellipticity effects. 
Figure V.C.4 compares the signals one would measure, $I(y)$, at a number of axial locations with the corresponding $I^{\prime}(u)$. The broadening of the measured signals is due solely to the change in ellipticity of the magnetic field, and when this effect is compensated for the signals lie on top of each other. Using $I^{\prime}$ as the signal and $u$ as the independent variable, an Abel inversion can be performed to obtain the plasma emissivity.

\section{Abel Inversion Technique}

To obtain the plasma emissivity from the corrected intensity values, an Abel inversion must be performed. Numerous Abel inversion techniques are in common practice; two methods considered for use in the analysis of the camera data are discussed below. One approach to the problem is to solve the integral equation,

$$
I(y)=2 \int_{y}^{\left.r_{1}\right)} \frac{\epsilon(r) r d r}{\sqrt{r^{2}-y^{2}}}
$$

for the emissivity, $\epsilon$, given the detected intensity, $I$. Once it has been finitedifferenced, the equation can be solved by either matrix inversion or least squares minimization. Because this method is very sensitive to variations in the measured intensities, additional restrictions, sometimes called "regularizations," must be imposed to obtain a solution free from rapid oscillations. ${ }^{1}$ This method is readily applicable to problems in which the number of detected signals is large $(>20)$ and the associated errors are small $(\sim 1 \%)$. Another approach is to assume an analytic model for the emissivity, such as a parabola or gaussian, and then determine the set of model parameters 
Detected Signals at 5 axial locations-I(y)

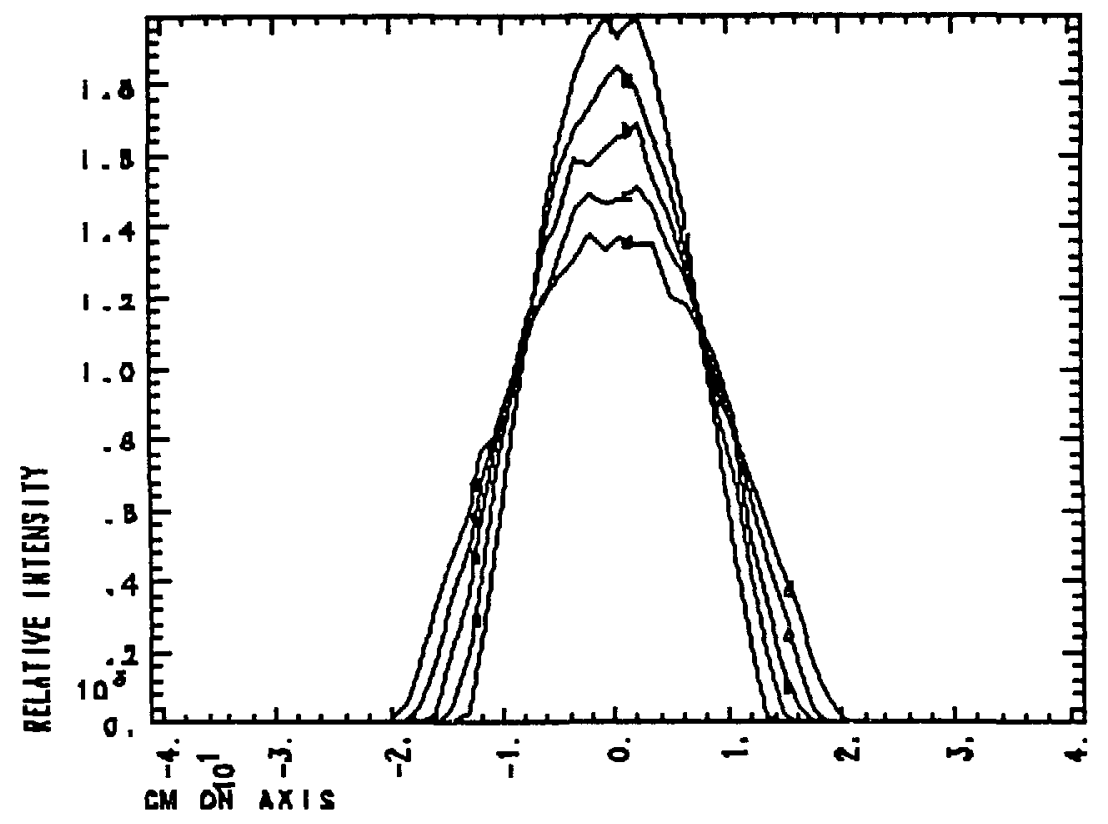

Signals with ellipticity effects removed-l'(u)

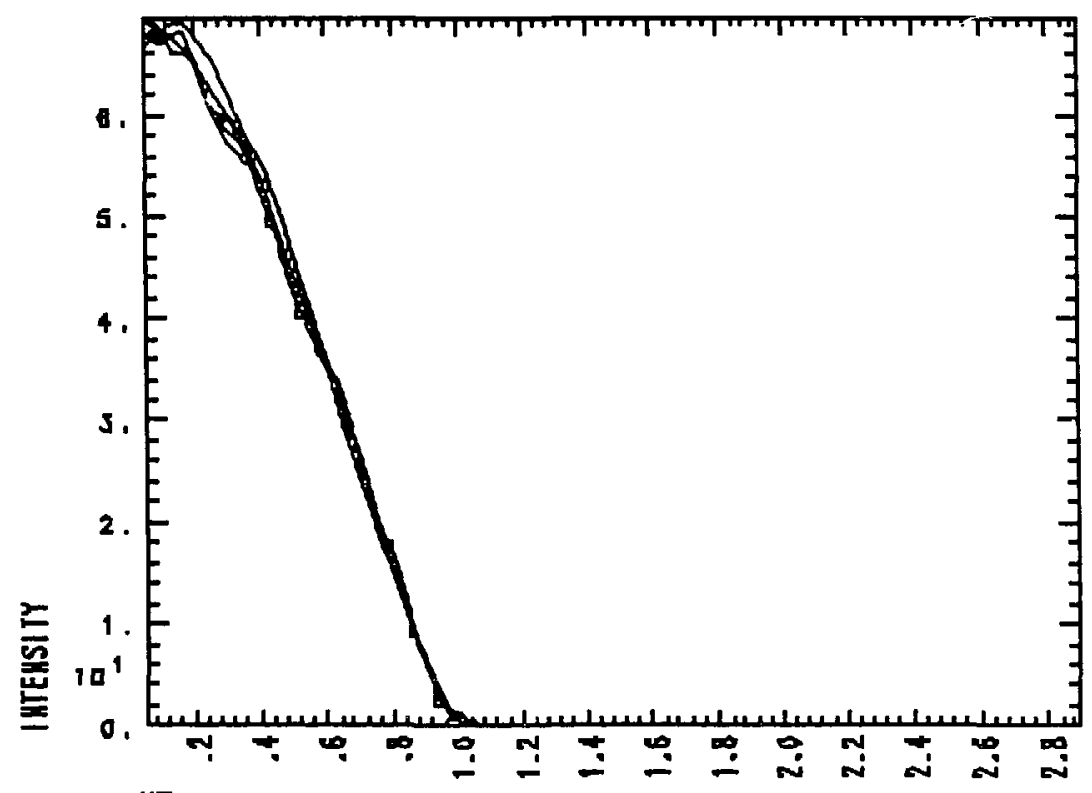

UT

Figure V.C.4. Detected signals, and signals corrected for plasma ellipticity. 
which best fit the experimental data. Because the uncertainties in the experimental data were relatively large $(\sim 10 \%)$, the second approach was used to calculate the $x$-ray emissivity. (For examples of fits, see section $B$, chapter VIII.)

Typically the model used to fit the data had three free parameters; one corresponded to an amplitude, another to a radius, and the third to a uniform background level. A nonlinear least squares fit was performed using subroutines from the NAG FORTRAN Library. ${ }^{2}$ In particular, the subroutine E04FC was used to minimize the residuals.

\section{E. Error Analysis}

Statistical variations are the main source of error. For this reason the fluctuations in the number of photons that strike a given region of the detector crystal, $N$ are of order $\sqrt{N}$. Because the detector is absolutely calibrated, an estimate of the number of photons absorbed in the scintillator can be determined given the x-ray spectrum measured by the spectrometers. If $I$ is the measured intensity on the film and $a$ is the area of the film over which this intensity was measured,

$$
I=\frac{g}{a} \times \sum_{i=1}^{N} E_{i}
$$

where $g$ is the gain of the detection system and the $E_{i}$ are the energies of the photons that struck the region of the detector that corresponds to the area $a$ on the film. Defining an average energy, $\bar{E}$

$$
\bar{E}=\sum_{i=1}^{N} E_{i} / N
$$


so that

$$
I=\frac{g}{a} \widetilde{E} N
$$

one can define the deviation in $I, \sigma_{I}$, as

$$
\sigma_{I}=\frac{g}{a} \bar{E} \sqrt{N}
$$

Since the x-ray signals can be well fit by an exponential (or sum of two exponentials), $\bar{E}$ can be found by performing the integrals

$$
\bar{E}=\int_{E_{\text {min }}}^{E_{m i t x}} f(E) \exp \left(-\frac{E}{T_{e}}\right) d E / \int_{E_{\text {min }}}^{E_{m i x x}} f(E) \frac{\exp \left(-\frac{E}{T_{e}}\right)}{E} d E
$$

where $f(E)$ is the response of the detector as a function of photon energy. Figure V.E.1 shows how $\vec{E}$ varies with $T_{e}$ for .5 and $3 \mathrm{~mm}$ CsI crystal thicknesses and an $8 \mathrm{mil} \mathrm{Al}$ window. For these filter/scintillator combinations $10<\bar{E}<70$ for $5<T_{e}<500 \mathrm{keV}$. Now $\sigma_{I}$ can be found from

$$
\sigma_{I}=\sqrt{\frac{g}{a} \bar{E} I} \frac{\mathrm{erg}}{\mathrm{cm}^{2}}
$$

Since the signals corresponding to each pixel, or area of the film that is digitized, are independent of each other, when they are summed the error in the sum is given by

$$
\sigma_{\text {total }}^{2}=\sum_{i=1}^{N} \sigma_{i}^{2}
$$

This means it is appropriate to keep track of the $\sigma_{I}^{2}$ values, and when any averaging is done over the image, the same averaging should be done over $\sigma_{I}^{2}$. Thus the error in the final result can be determined. 


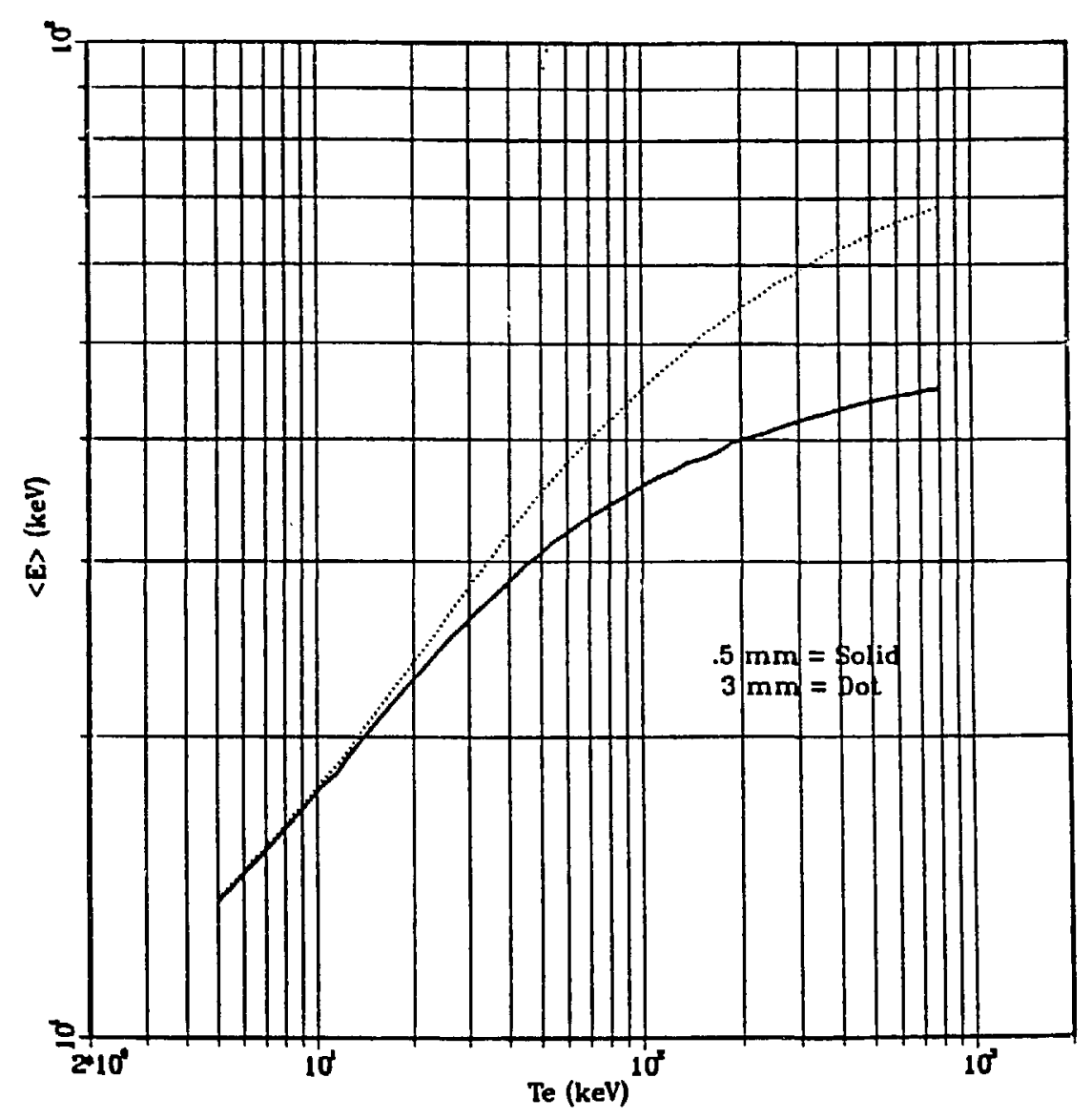

Figure V.E.1. $\overline{\mathrm{E}}$ versus $T_{\mathrm{e}}$ for .5 and $3 \mathrm{~mm} \mathrm{Csl}$ thicknesses (8 mil Al window). 
The sensitivity of the fit parameters to experimental error was determined by performing the fit on random deviates of the measured intensities. Deviates were generated according to a gaussian distribution, with the standard deviation of a detected signal given by its $\sigma_{I}$ value. This is a straight-forward way to generate error bars for the fit parameters. After approximately fifty fits had been performed, the parameter estimates (given by the average of all the fit results) and their associated deviations usually ceased to vary and were therefore accepted. 


\section{Chapter VI}

\section{Detected Images}

\section{A. Introduction}

This chapter is an overview of the different types of $\mathrm{x}$-ray emission observed in the TMX-U west end cell. Subsequent chapters will deal with the individual emission processes in more detail.

A number of features can be seen in a typical image of the west end cell. A contour and pseudo 3D plot of an image is shown in figure VI.A.1. The machine axis ( $z$ ) runs from left (east) to right (west), while the top of the plot corresponds to north and the bottom to south. The second harmonic microwave heating beam is coupled to the plasma from the north side. On the left side of the figure the volume $x$-ray emission is cut off by a magnet coil, and to the right it is limited by the edge of the scintillator. At an axial value of about 546, the diamagnetic loop support attenuates emission from the plasma. Also indicated on the figure are bright sources of thick target emission corresponding to a location on the diamagnetic loop and also the tip of a radio frequency probe. These are the features typically seen in an $\mathrm{x}$-ray image of the west end cell. The ring that encircles the image is due to scattering of scintillated light from the sides of the crystal. This particular shot had short second harmonic microwave heating and during the time of the exposure the microwaves were off. For this reason, the emission from the north DML location is relatively weak. To obtain an image during the heating pulse, the bright thick target emission had to be masked off with a 

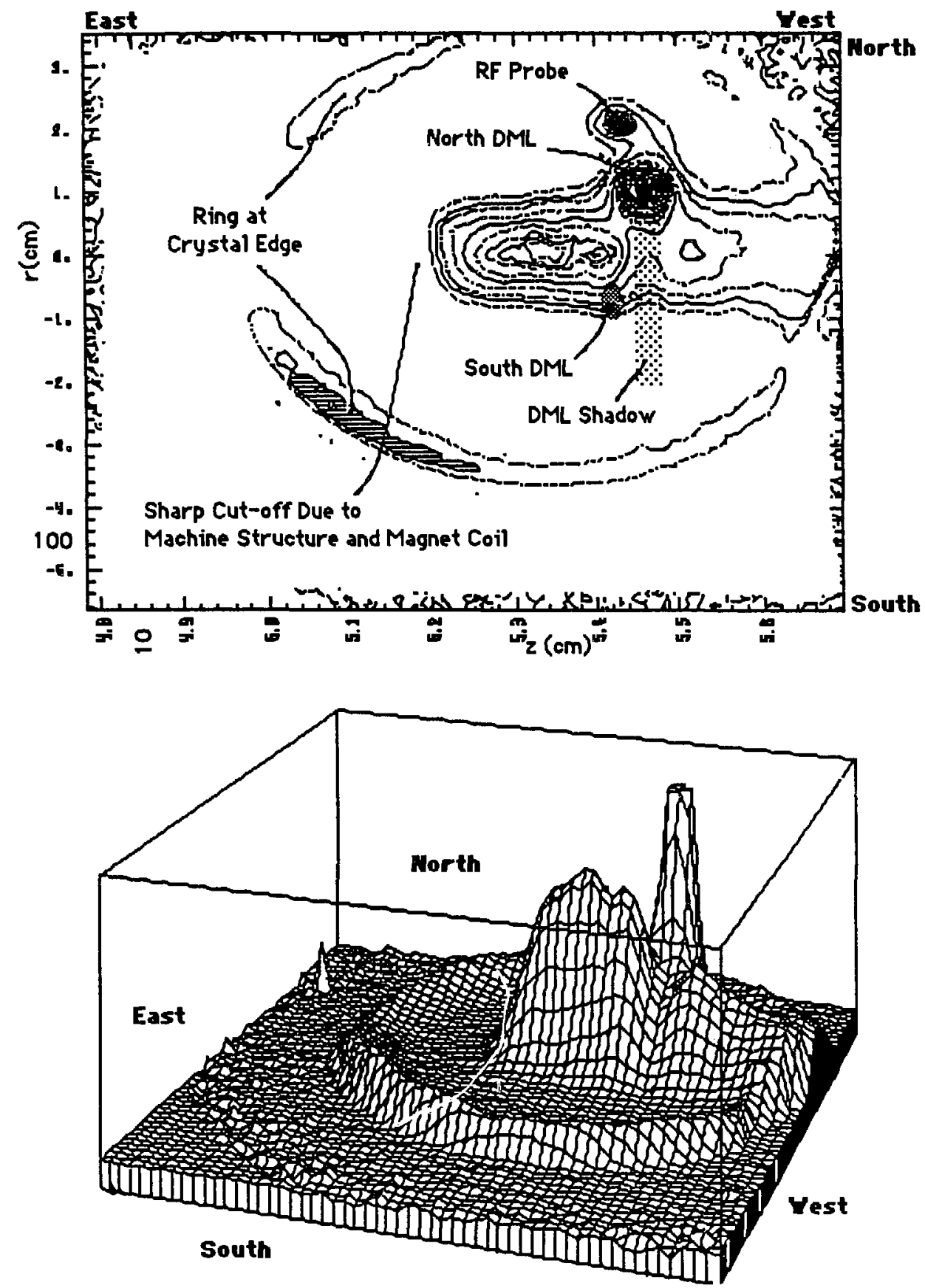

Figure VI.A.1. Sources of $\mathrm{x}$-ray emission in the west end cell of TMX-Upgrade. Axis of machine runs from east to west; apparent reversal due to the fact that pinhole image is coupled to film with lens. Brightest $x$-ray source is north $D M L$ spot; clear attentuation of volume emission by the DML itself. Data shown is film exposure. 
sheet of $\mathrm{Pb}$. An example of an image taken this way is given in the next section.

\section{B. Volume Emission}

The brightest volume emissivities were produced on shots which employed neutral beam injection. This is because impurities, such as oxygen and nitrogen, were injected at high energies along with the primary beam species-typically deuterium or hydrogen. As discussed earlier (chapter II, section $B$ ), it is a reasonable approximation to weight the impurity concentrations by their bare charge. Because the free-free bremsstrahlung cross-section is proportional to $Z^{2}$, one percent of $\mathrm{O}$ will increase the volume emission by a factor of 1.64 . The $O$ and $N$ concentrations of .009 and .005 respectively have been estimated from extreme ultraviolet spectrometer measurements. ${ }^{1}$ Thus the bremsstrahlung emission typically would be increased by over $80 \%$ during neutral beam injection.

It is reasonable to assume that the impurities have a uniform concentration throughout the plasma. ${ }^{2}$ This is because they are injected at the jeam energy of $10 \mathrm{keV}$, and can therefore penetrate the plasma well. For this reason the measured $\mathrm{x}$-ray emissivity is taken to be proportional to the square of the mirror-trapped electron density (see chapter II, section B).

\section{Thick Target Emissions}

Thick target emission was primarily seen from three distinct locations, two spots on the diamagnetic loop (DML) which are each about $115 \mathrm{~cm}$ from 
the machine axis and the tip of a radio frequency probe that is typically inserted to about $25 \mathrm{~cm}$ from the machine axis. The emission from the rf probe can be explained by its proximity to the main plasma, but the spots on the DML, which are typically much stronger, should not be present. Magnetic field gradients cause the electrons to drift azimuthally about the axis, and they should strike the of probe long before they reach the diamagnetic loop. An explanation for these anomalous emissions is given in the next section.

\section{Scintillator Ring}

The ring that circles the image results from light trapped in the scintillator by total internal reflection at the interface with the fiber optic. At the edge of the crystal the light can scatter diffusely and thus escape. This problem was discussed in an earlier section. The width of the ring (as previously discussed in chapter III, section B), is somewhat larger than expected from the theoretical treatment applied, and varies from case to case. Data affected by this ring is simply not used in the determination of the plasma shape.

\section{E. Summary}

The plasma volume emission, the thick target emission from the rf probe, and the ring due to diffuse scattering at the crystal edge are all well understood, and relatively simple to explain. The origin of the two most intense sources of $x$ rays during the heating phase of a shot-the north and south locations on the diamagnetic loop-have a more complex expanation. The 
origin of these thick target emissions, and their effect on the performance of the experiment is the subject of the next chapter. 


\section{Chapter VII \\ Thick Target Emissions}

\section{A. Introduction}

Thick target emissions produced by microwave-heated electrons striking the diamagnetic loop have been observed in the west end cell of TMX-U. Locations of these observed emissions are shown in figure VII.A.1. It is important to determine exactly how and where these electrons are produced. If they can be characterized by the same temperatures one typically measures with the $\mathrm{x}$-ray spectrometer systems, the magnitude of the thi $k$ target emission would be consistent with significant electron power loss. Numerical analysis of the electron drifts indicate that these emissions are due to

electrons heated at radii larger than about $20 \mathrm{~cm}$. Also, the magnitude of the emission increases with time during a plasma shot, which would indicate more microwave power is being absorbed at large radii late in the heating pulse. During this time a significant fraction of the microwave power may be coupled to the class of poorly confined electrons which produce the strong thick target emission.

\section{B. Experimental Data}

There is strong empirical evidence that the thick target emissions are due to electrons heated at large radii. For example (1) the electron drifts are consistent with the observed symmetry of the emission, (2) the presence of the main plasma reduces the emission at one location as compared to 
The axial location is $\mathrm{z}=542$.

The spacing between contours is 40 Gauss.

The spacing between major ticks is $10 \mathrm{~cm}$.

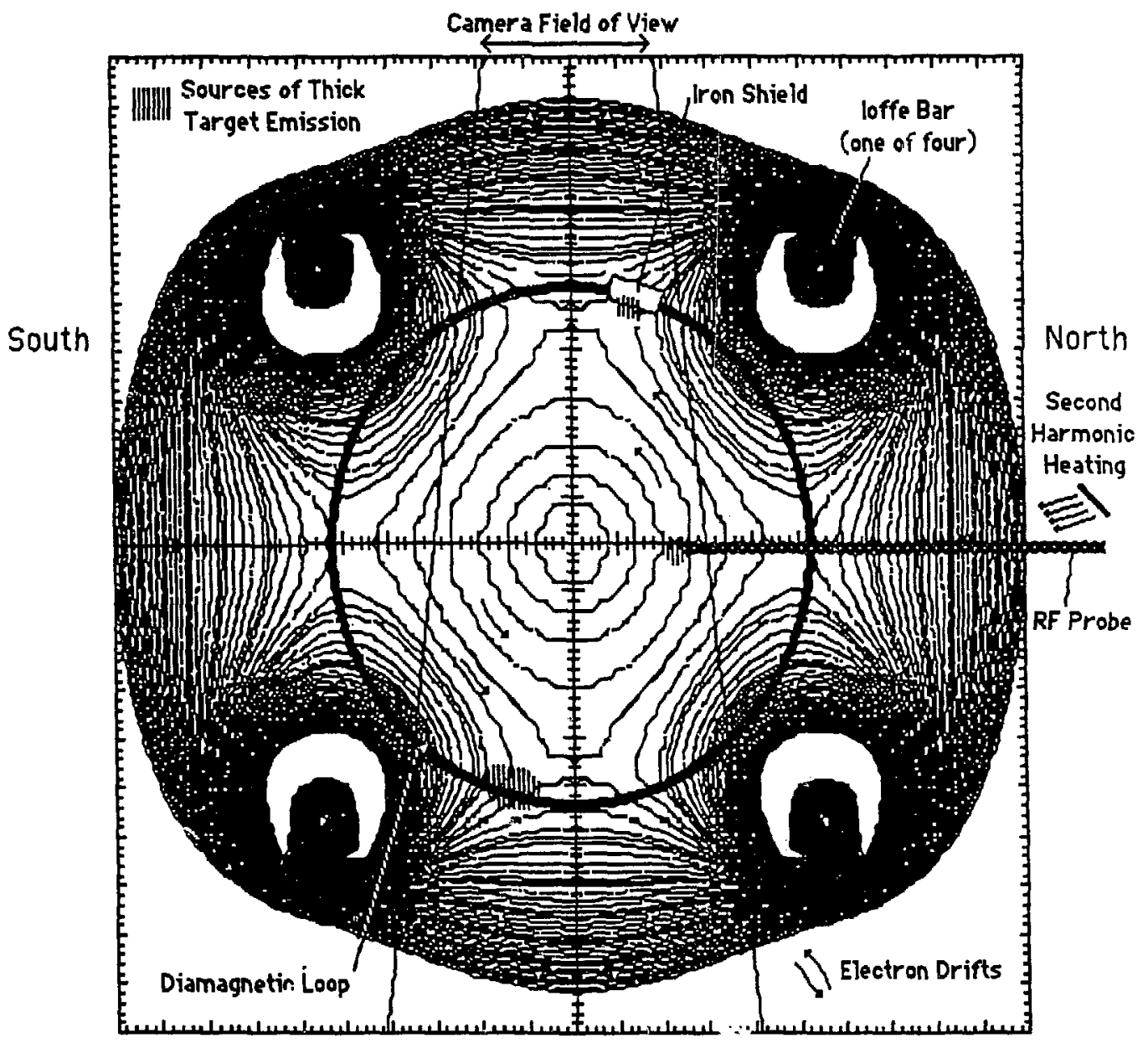

Figure VII.A.1. Sources of thick target emission with $|B|$ contours. The Ioffe magnet coil provides the quadrapole field needed for a minimum $|\mathbf{B}|$ configuration. 
the other, (3) there is an abrupt change in the emission when the plasma potential changes and also when the magnet currents decay, and (4) the location of the emission depends on the magnetic field case. Also, the level of thick target $\mathrm{x}$-ray emission is low during the first $17 \mathrm{msec}$ of microwave heating but high thereafter. This is consistent with increased radial electron losses late in the microwave heating pulse.

Electrons drift because of a magnetic field gradient perpendicular to the magnetic field. The magnitude and direction of the drift velocity, in the guiding center approximation, is given in the nonrelativistic limit by

$$
\mathbf{v}_{d r i f t}=c \frac{m v_{\perp}^{2}}{2 q B} \frac{\mathbf{B} \times \nabla B}{B^{2}}
$$

where $q, m$, and $v_{\perp}$ are the particle charge, mass, and velocity perpendicular to the magnetic field, respectively, $c$ is the speed of light, and $\mathbf{B}$ is the magnetic field vector. All quantities are in cgs units. ${ }^{1}$ Note that the drift velocity is proportional to the particle energy, and that the direction of the drift, for electrons, is given by $\nabla B \times \mathbf{B}$.

The drift that a particle executes while in a magnetic mirror also depends on its pitch angle. The pitch angle, $\theta_{\text {pitch }}$, is defined as

$$
\theta_{\text {pitch }}=\arctan \frac{v_{\perp \text { min }}}{v_{\| \min }}
$$

where $v_{\perp \text { min }}\left(v_{\| \min }\right)$ is the particle velocity perpendicular (parallel) to the magnetic field at the magnetic minimum of the mirror. The pitch angle of a particle determines the axial location at which it will mirror, and because 
the topology of the magnetic field varies axially, particles with different pitch angles will execute different drifts.

The electron drifts in the west end cell were calculated using a computer program. The machine coordinate system of TMX-Upgrade is oriented so that the $x$-axis points south, the $y$-axis points up, and the $z$-axis points west. This coordinate system is referred to throughout the remainder of this chapter. Given a magnet geometry and associated currents, the program, GCDRIFT, traces particle trajectories in the guiding center approximation. ${ }^{2}$ This approximation is valid if the variation of the magnetic field over a particle gyroradius is small compared to the magnitude of the field. ${ }^{3}$ Time required for a $100 \mathrm{keV}$ electron to drift $360^{\circ}$ azimuthally around the machine axis at a radius of $9 \mathrm{~cm}$, as calculated by GCDRIFT, is about 70 microseconds, which is in reasonable agreement $(\sim 10 \%)$ with another code. ${ }^{4}$

Results of the guiding center code indicate that confinement degrades with increasing radius and decreasing pitch angle. These conclusions are summarized in figure VII.B.1. The top plot shows that a particle with a given pitch angle is confined if launched at one radius, but lost if started at a larger one. The lower plot indicates that particles starting at the same radius are confined only if their pitch angle is sufficiently large. The particle trajectories are consistent with the locations of observed thick target emission; electrons drift up (down) on the north (south) until they strike the diamagnetic loop. Also, unconfined drift surfaces exist which can carry electrons in front of the If probe and into the diamagnetic loop. This explains why the diamagnetic 
loop emission locations are much brighter than the tip of the rf probe during the heating pulse.

To understand how the pitch angle required for confinement increases with radius, it is helpful to study simulated trajectories of positrons launched with different pitch angles from the location of brightest thick target emission on the diamagnetic loop. The trajectory of a positron moving forward is equivalent to that of an electron moving backward in time, so the location where the electrons started near the $x$-axis can be determined in this way. Since confinement improves with pitch angle and deteriorates with radius, unconfined particles with large pitch angles must have been produced at large radii. Examples of drifts are shown in figure VII.B.2. Also shown is the way that the pitch angle required for good drifts increases with the $x$-axis intercept of the particle. Thus electrons can be lost due to poor drifts for two reasons-their guiding center is too far from the machine axis or their pitch angle is too small.

As the main plasma builds up, the rate at which the two emission locations increase in intensity are different. See figure VII.B.3. This behavior can be explained by the fact that the microwave beam is attenuated as it traverses the plasma. Early in time, when the electron density is low, emission from the north and south locations should be about the same. The detected signal on the north is less than that on the south because $\mathrm{x}$ rays produced on the north must pass through a $\sim 25 \mathrm{mil} F e$ shield in order to reach the camera. Electrons strike the side of the shield closest to the plasma, and only 

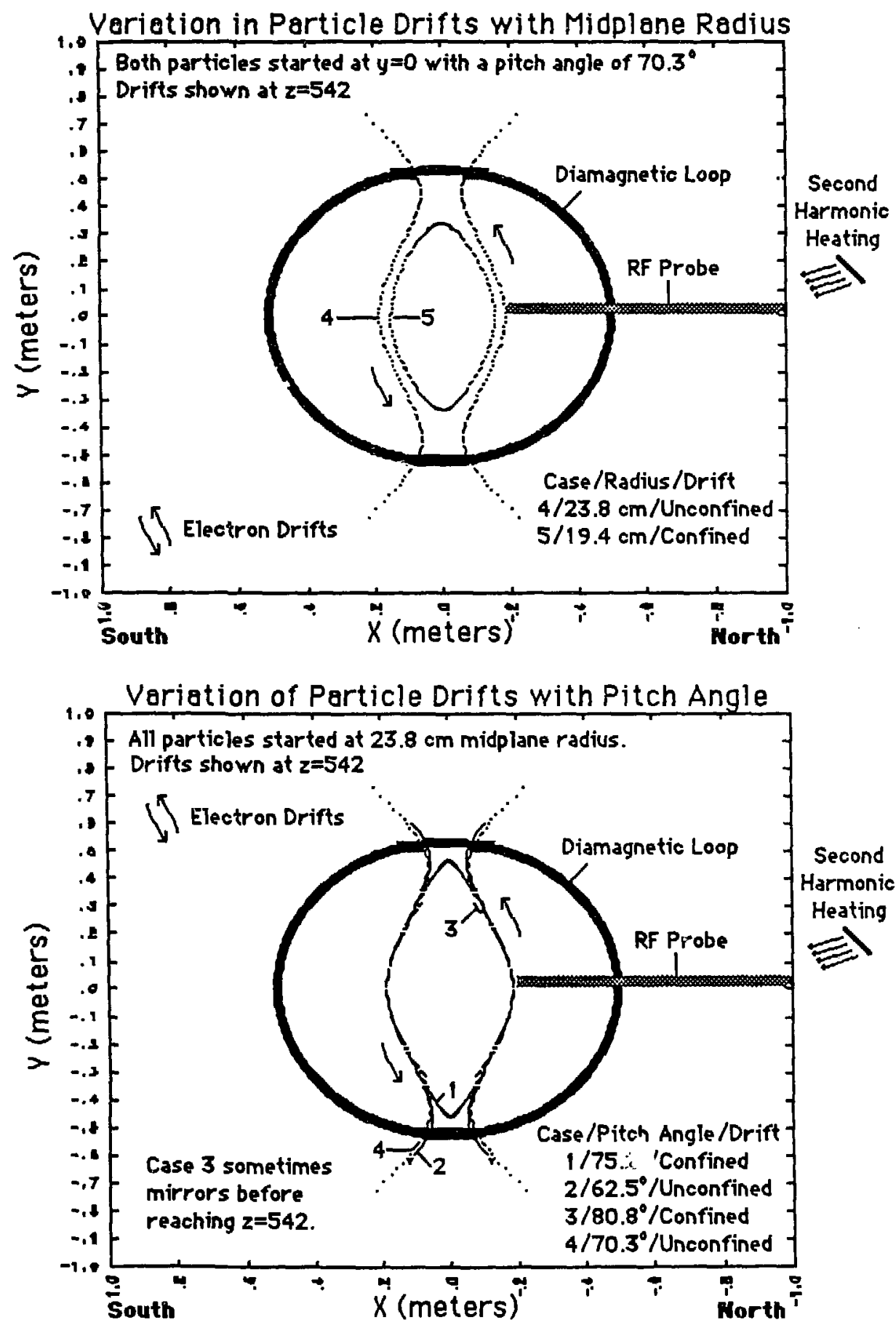

Figure VII.B.1. Electron guiding center drifts in TMX-Upgrade west end cell. 

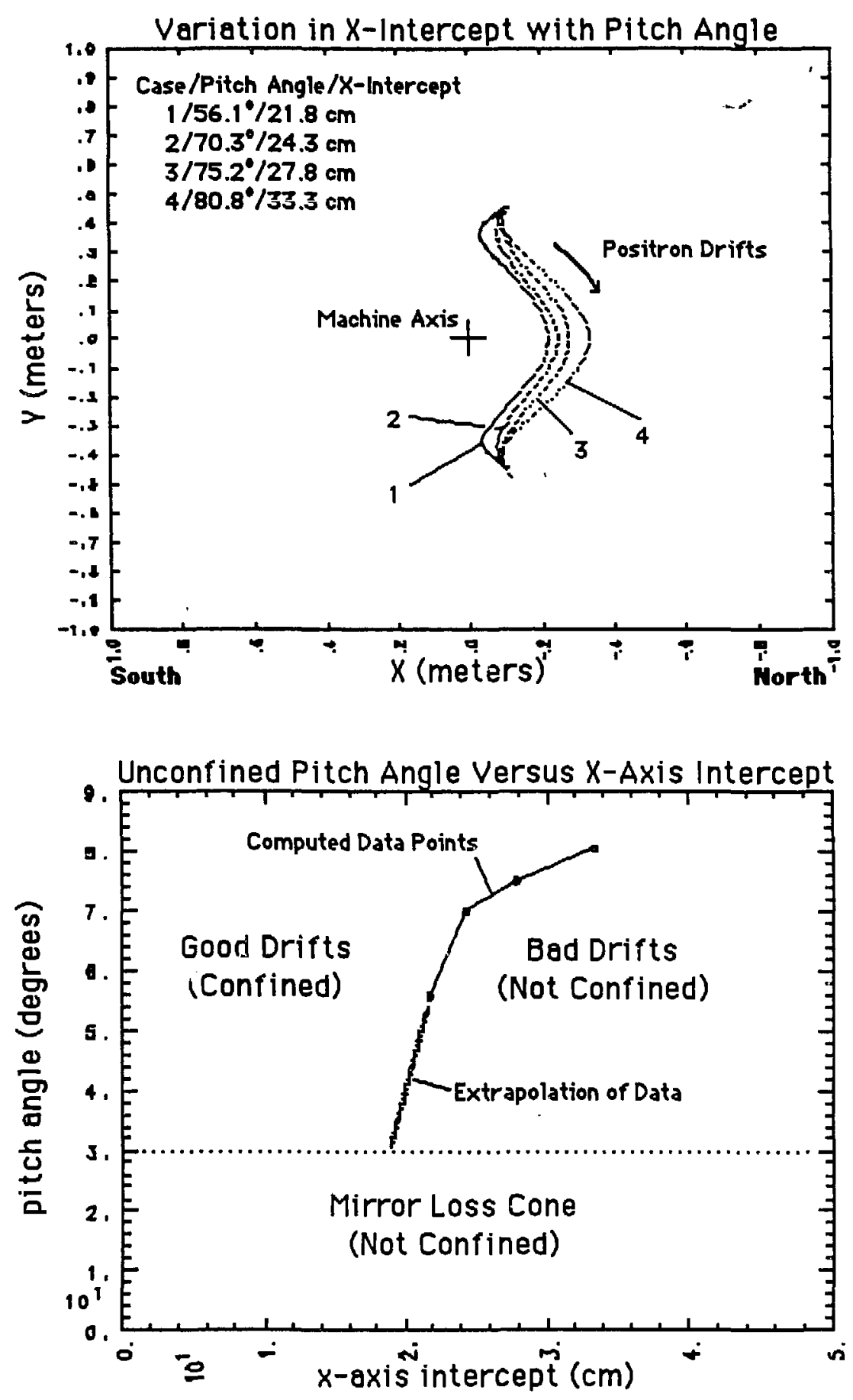

Figure VII.B.2. Pitch angle required for confinement increases with $x$-axis intercept. 
$\mathrm{x}$ rays which pass through it can be detected. The shield was p'aced over the diamagnetic loop to prevent it from being damaged. As the density of the main plasma increases, the rate of rise of the south spot is much le ; than that for the north spot. This can be attributed to the fact that the plasma begins to absorb a great deal of the microwave power. It is this absorption that prevents damage to the loop on the south, and the lack of it which has in the past allowed the spot on the north to be melted.

Immediately after the heating systems turn off and the main plasma is rapidly lost there is typically a strong increase in the thick target emission. During this time the plasma potential is changing from near ground or positive to negative because the mirror-trapped electrons are the most well-confined class of particles. During the high density phase of a shot, Extreme UV measurements of $\mathrm{Ti}$ line emission are consistent with the presence of a radially directed electric field (due to a positive potential with respect to the machine wall) at the plasma edge..$^{5}$ The fact that $x$-ray line emission characteristic of several high $\mathrm{Z}$ impurities is seen during the afterglow but not during the high density phase of the shot supports the hypothesis that the potential becomes more negative (see the discussion of heavy impurity line emission in chapter II, section B). A negative potential would enable the cold impurities to diffuse into the plasma core; it would also expel electrons, increasing the thick target emission.

As the magnet currents decay the emission again increases abruptly. At this time the location of thick target emission broadens and moves out ra- 
Time Histo, $y$ of Thick Target Emission from Two Spots on the Diamagnetic Loop Indicate Increasing Plasma Absorption

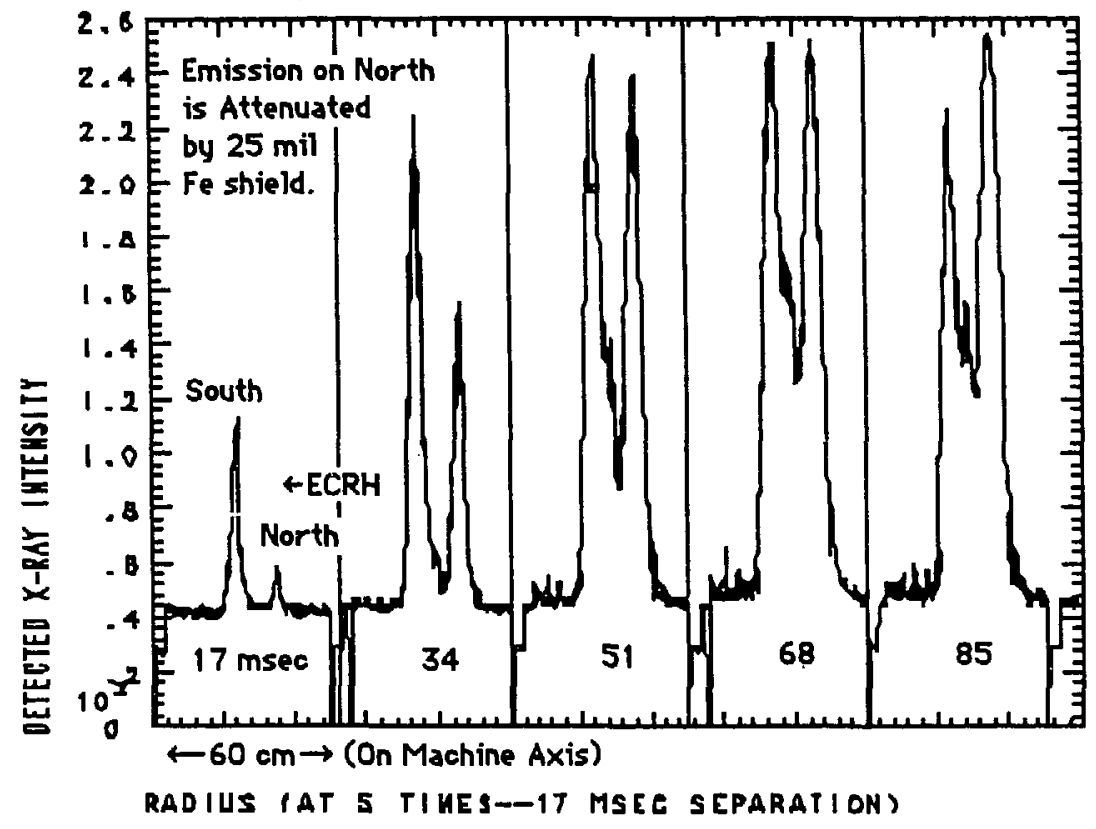

Figure VII.B.3. Time variation of thick target emission during heating pulse. Emission increases more strongly on side where microwaves are fed in. 
dially, which is shown in figure VII.B.4. The Ioffe coil, which provides the quadrupole fields, decays before the circular magnets. This means that the |B| surfaces will become more circular and the location where the electron drifts intersect the diamagnetic loop should move toward the $x$-axis (from point 1 to point 2 , as shown in the figure) and this is what is seen experimentally. Before the magnet currents decay, the emission is very low because the energetic mirror-trapped electrons scatter in pitch angle relatively infrequently. As the magnetic field decreases, conservation of magnetic moment implies that $v_{\perp}$ must also decrease, and (since the particle energy is constant) $\theta_{\text {pitch }}$ also decreases. For this reason, electrons with pitch angles large enough to be confined when the magnetic field is high, will be lost when it decays.

The location of the thick target emission also depends on the magnetic field configuration. This is shown in figures VII.B.5-7. A set of magnet currents similar to those designated by the TMX-U UPHP30 magnetic field configuration was typically used during standard TMX-U operation, while the SAI configuration was specially designed to have reduced radial transport. ${ }^{6}$ The difference in the magnetic field produced by the SAI configuration caused the drift surface to change so the point where it intersected the diamagnetic loop moved up at the southern location. Also, the energy of the electrons striking the north location was reduced to the point that no emission was seen through a $500 \mathrm{mil} \mathrm{Pb}$ sheet. This sheet was employed to attenuate the very strong thick target emission that was typically produced on the north during standard TMX-U operation. 


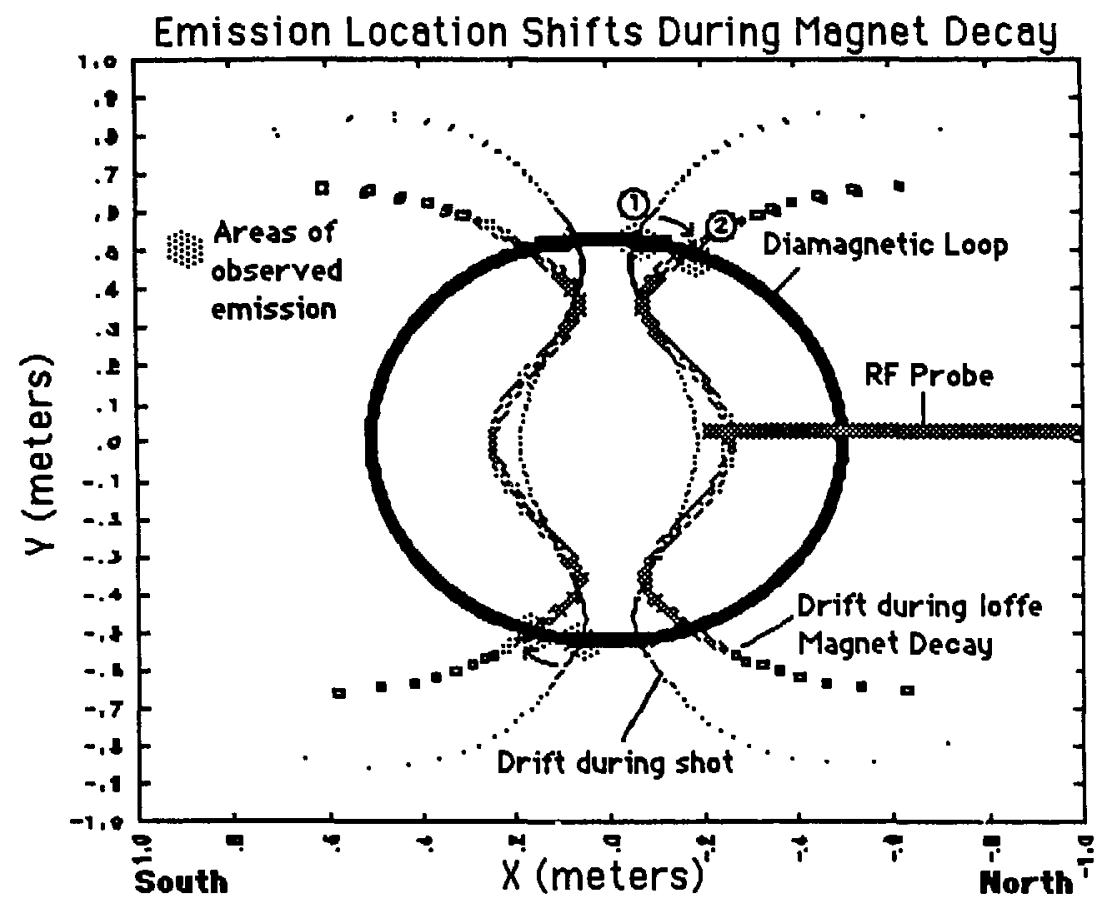

Thick Target Emission Increases and Moves Out in

Radius as the Magnet Currents Decay

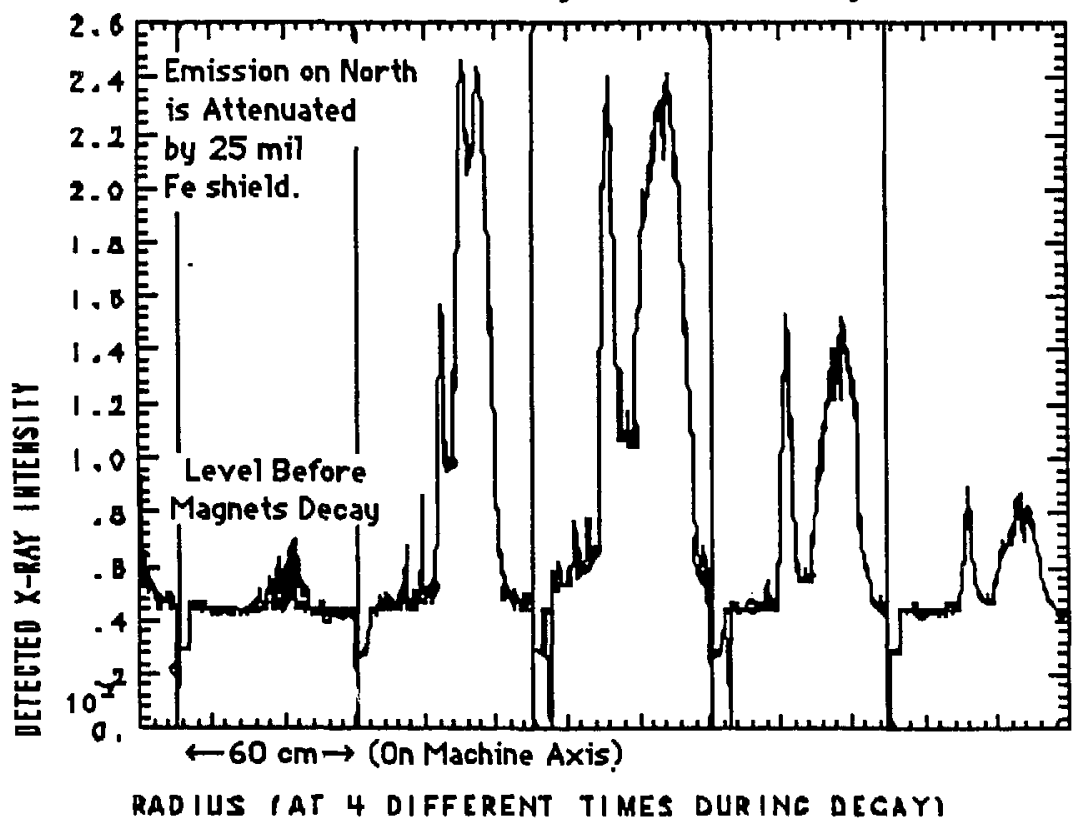

Figure VI.B.4. Thick target emission during magnet decay. 

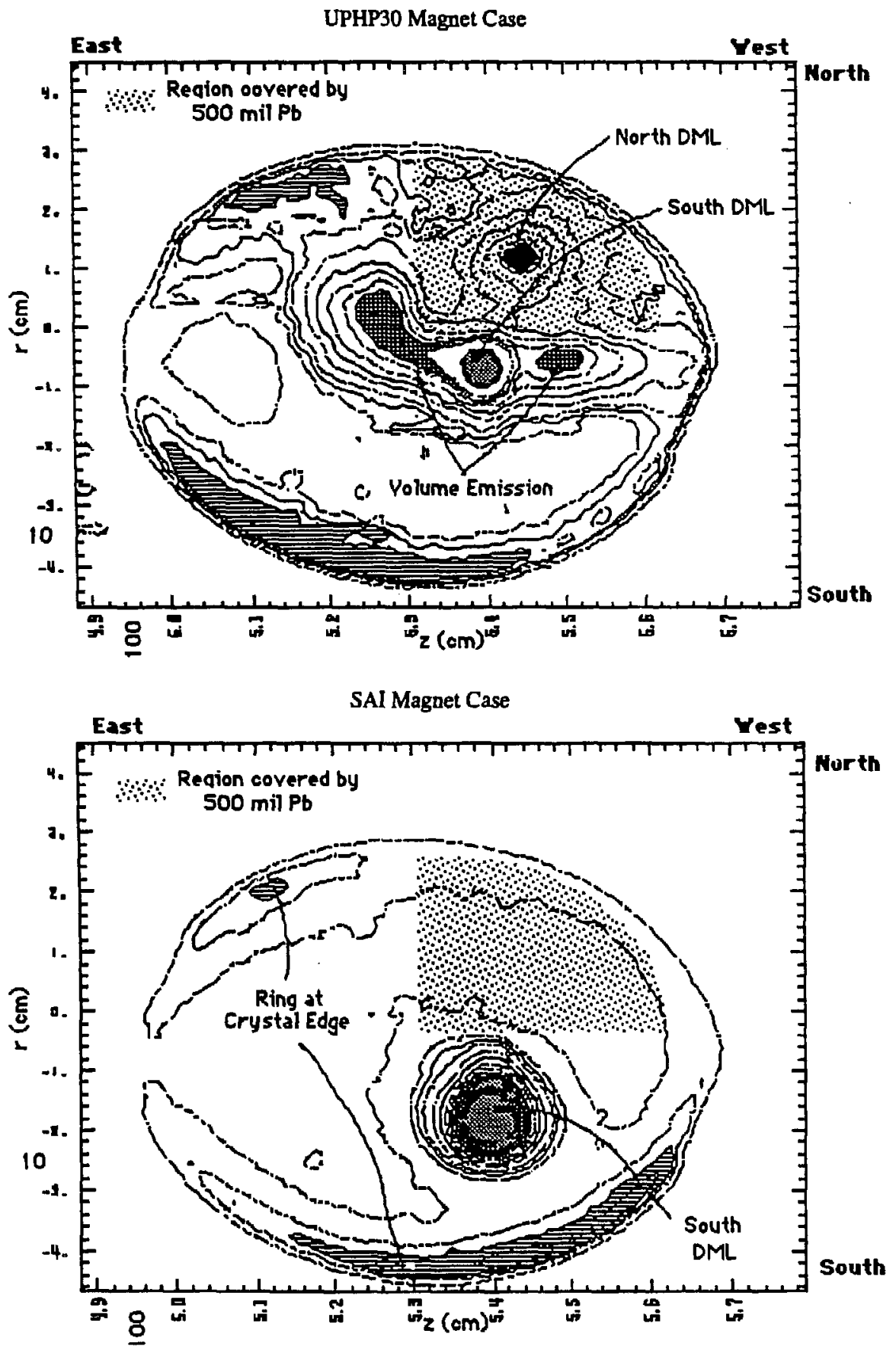

Figure VII.B.5. Comparison of thick target emission for standard (UPHP30) versus SAI magnetic field case.Axis of machine runs from east to west; apparent reversal due to the fact that pinhole image is coupled to film with lens. No volume emission seen for SAI, but very strong thick target emission fron south DML consistent with weak volume absorption.

Data shown is film density. 

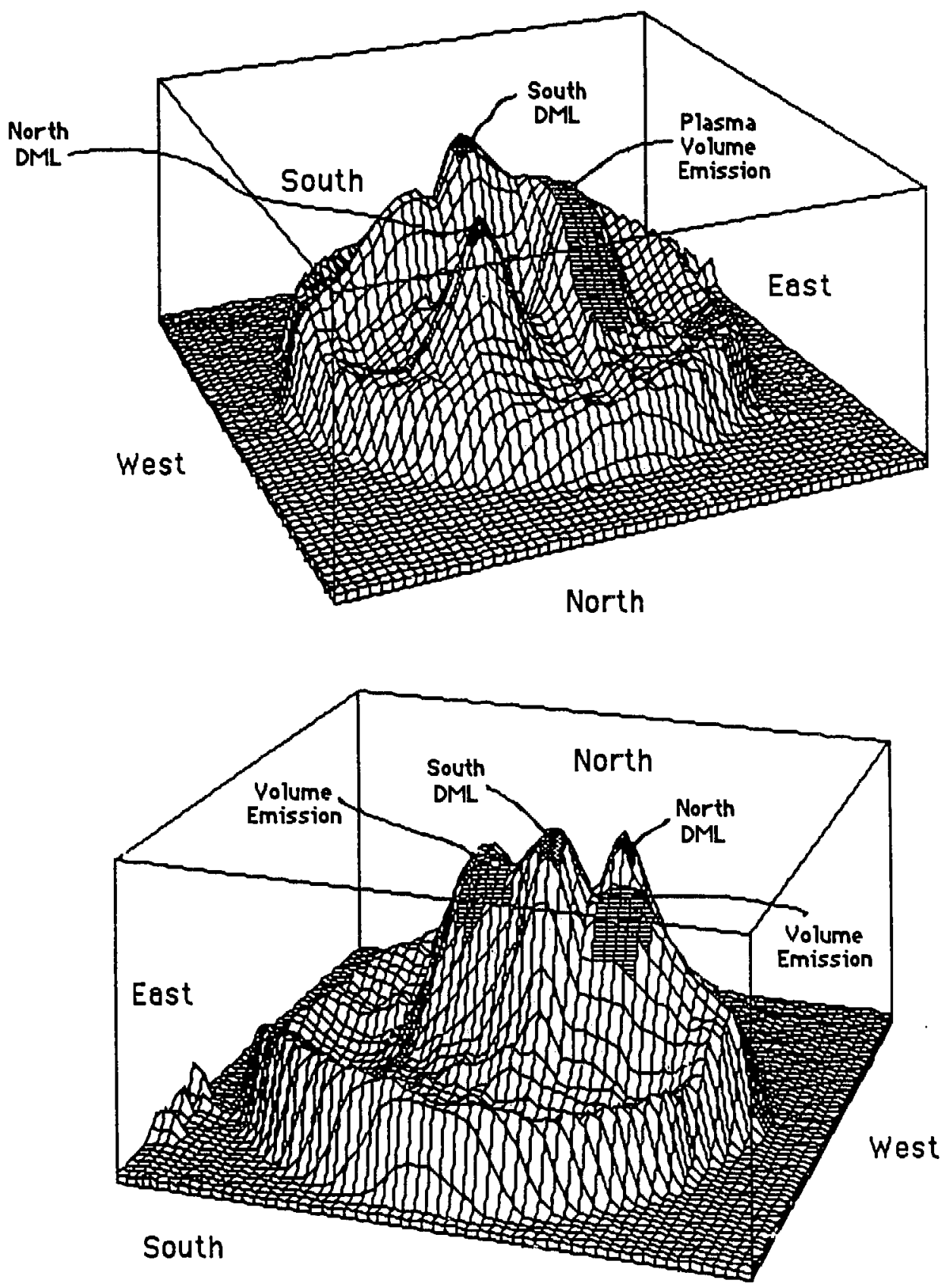

Figure VII.B.6. Thick target emission for the standard magnetic field case (UPHP30). Axis of machine runs from east to west; apparent reversal due to the fact that pinhole image is coupled to film with lens. Volume emission is strongly attenuated by a 500 mil Pb thickness, but north $\mathrm{DML}$ spot can still be clearly seen. Data shown is film density. 

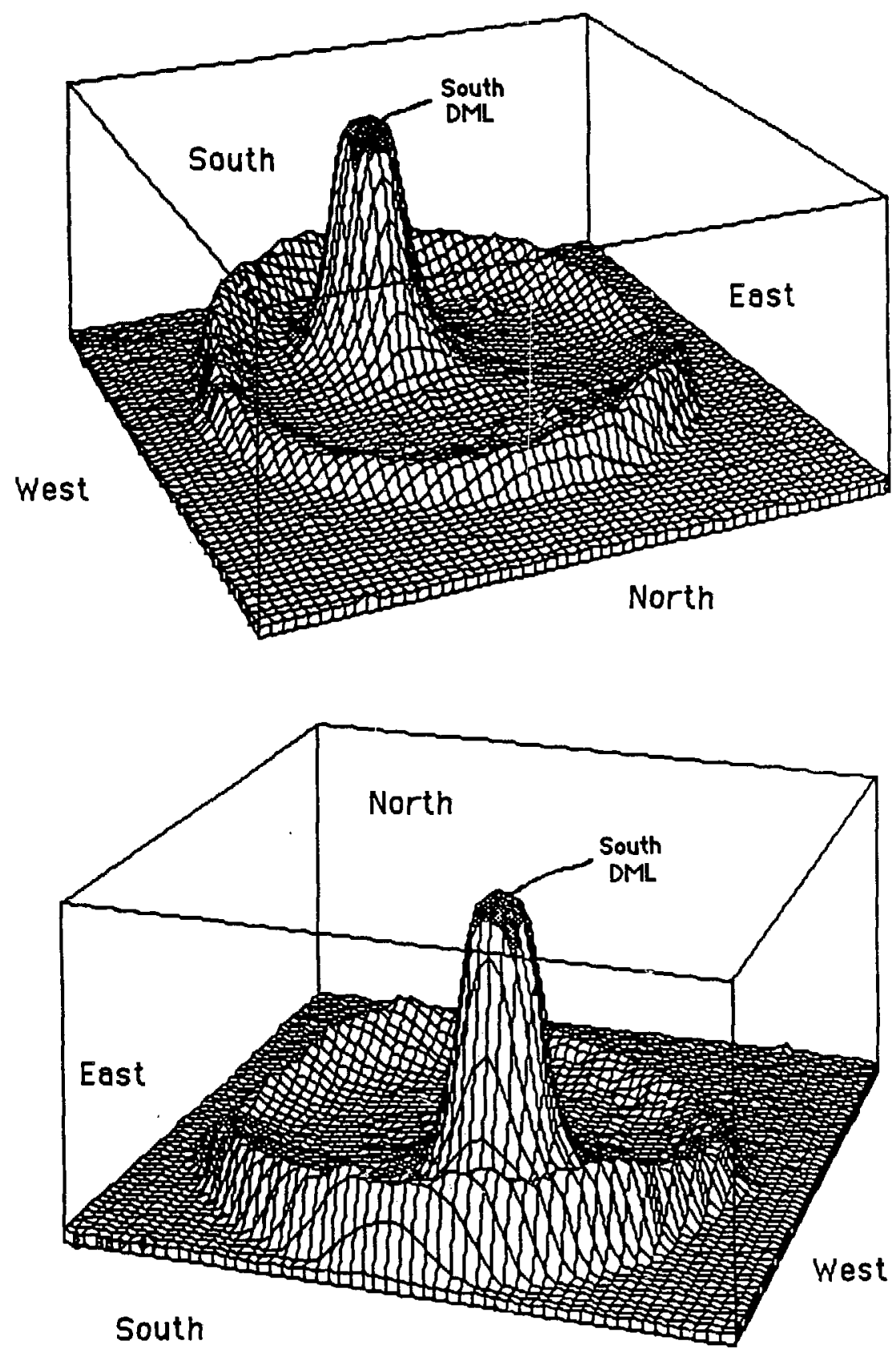

Figure VII.B.7. Thick target emission for the SAI magnetic field case. Axis of machine runs from east to west; apparent reversal due to the fact that pinhole image is coupled to film with lens. The only distinct emission is from the south spot on the DML; no plasma volume emission is seen. Data shown is film density. 
For a number of shots, the absolute magnitude of the thick target emission from the brighter north spot was analyzed. The detected emission versus time is plotted in figure VII.B.8. Points with the same gas fueling rate are crnnected. In all cases the data indicates that the emission is low early in a shot, when the microwave heating efficiency is high, and high later, when the efficiency is low. Thus low microwave heating efficiency is correlated with increased thick target $\mathrm{x}$-ray emission due to radial electron losses. In chapter IX, variations in heating efficiency are discussed in greater detail.

\section{Discussion}

The location and time evolution of the thick target emission are consistent with heating and the subsequent loss of electrons at large radii-between 19 and $25 \mathrm{~cm}$ mapped to the plug midplane. Electrons with insufficiently large pitch angles will rapidly (in $\sim 30$ microseconds) drift into the diamagnetic loop and be lost. Those with large pitch angles are confined until they scatter sufficiently in pitch angle.

The fact that the iron shield on the diamagnetic loop is melted can be used to estimate the electron power loss. Flectron beams have been used for welding for a number of years. ${ }^{7}$ For example, in the case of 302 stainless steel, a penetration of 17 mils at a welding rate of 27 inches per minute can be obtained with a beam diameter of about 10 mils and power of 100 watts ( $a$ $100 \mathrm{kV}$ potential accelerates the electrons into the target). This corresponds to a beam intensity of about $200 \mathrm{~kW} / \mathrm{cm}^{2}$ at the workpiece. 

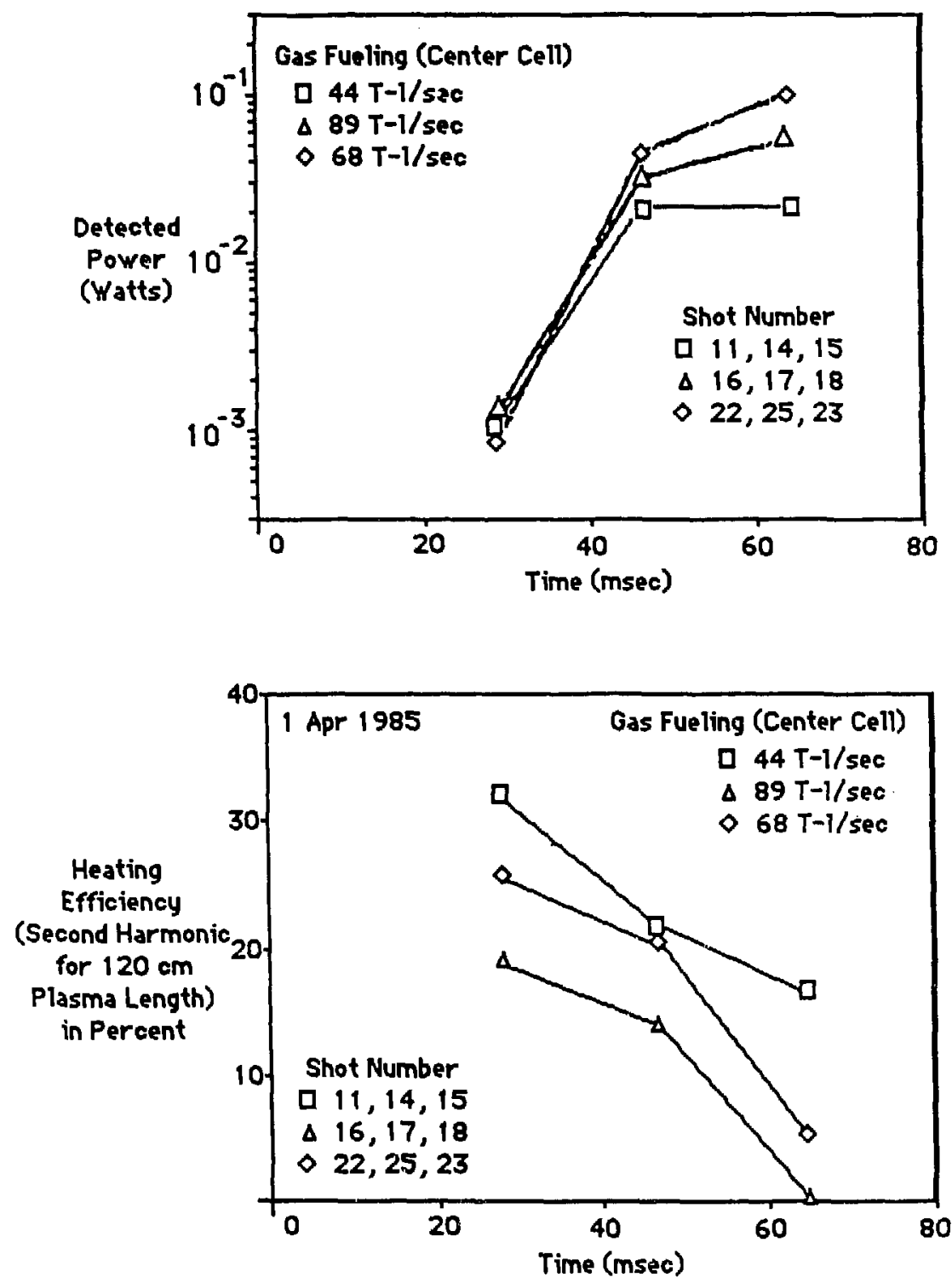

Figure VII.B.8. Time variation of thick target emission and microwave heating efficiency. 
An estimate of the electron scrape off length (the distance a particle drifts radially during the time it travels from one axial turning point to the other) is needed to determine the area of power deposition on the iron shield. For a $100 \mathrm{keV}$ electron, the scrape off length (as determined from the GCDRIFT runs) is about $.092 \mathrm{~cm}$. This length scales as the ratio of the drift velocity to bounce frequency, and is thus proportional to the square root of the electron energy, $W^{\frac{1}{2}}$ (the drift velocity is discussed in the previous section). Using the $100 \mathrm{keV}$ result, one can obtain the average scrape off length, $l_{\text {scrape }}$, as a function of temperature by averaging over the distribution of electron energies in the stream, $F(W)$. The result is

$$
l_{\text {scrape }}=.0092 \times \frac{\int_{0}^{\infty} W^{\frac{1}{2}} F(W) d W}{\int_{0}^{\infty} F(W) d W}=.0122 \sqrt{T_{e}} \mathrm{~cm},
$$

where the electron energy, $W$, and the temperature, $T_{e}$, are both given in $\mathrm{keV}$. The width of the melted region on the shield is on the order of $2-3$ $\mathrm{cm}$, which means that the area of power deposition is about $.25 \mathrm{~cm}^{2}$ for an electron temperature of $100 \mathrm{keV}$.

Using the area of deposition, one can determine the radial power loss to the shield necessary to melt it. The electron power loss required to produce an intensity of $200 \mathrm{~kW} / \mathrm{cm}^{2}, P_{\text {melt }}$, is given by

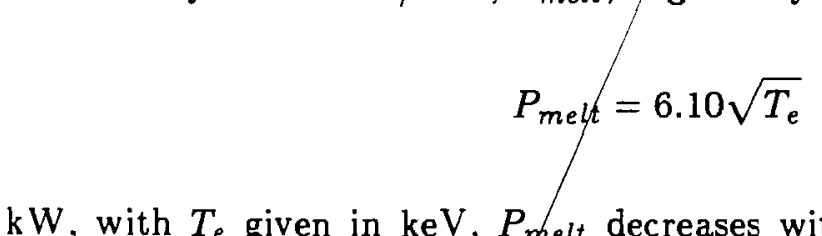

$\mathrm{kW}$, with $T_{e}$ given in $\mathrm{keV} . P_{\text {prelt }}$ decreases with electron temperature because the scrape off length becomes smaller. For an electron temperature characteristic of the warm electron component, $20 \mathrm{keV}$, the required power 
is $27.5 \mathrm{~kW}$, while for a hot component of $200 \mathrm{keV}$, the power is $85 \mathrm{~kW}$. Al:hough these are rough estimates, they indicate that losses comparable to the incident second harmonic power (typically $100-180 \mathrm{~kW}$ ) are required to produce the observed melting.

In order to estimate the radial electron power loss from the $\mathrm{x}$-ray emission, the thick target spectrum must be convoluted with the energy dependent response of the camera. The thick target spectrum, $I_{t h i c k}(E)$, is given in equation (II.B.4). The camera response is obtained by multiplying the filter transmission for a 125 mil $\mathrm{Pb}$ thickness $\left(\exp \left[-\mu_{P b}(E) x_{P b}\right]\right.$ where $\mu_{P b}$ is the linear attenuation coefficient for $\mathrm{Pb}$, and $x_{P b}$ is the filter thickness) by the absorption for $.5 \mathrm{~mm}$ of $\operatorname{CsI}\left(1-\exp \left[-\mu_{C \cdot s I}(E) x_{C \cdot s I}\right]\right.$, where $\mu_{C s I}$ is the linear attenuation coefficient for CsI, and $x_{C s I}$ is the scintillator thickness). The camera response, $R(E)$, is therefore

$$
\left.R(E)=\exp \left[-\mu_{P b}(E) x_{P b}\right] \times\left\{1-\exp \mid-\mu_{C s I}(E) x_{C s I}\right]\right\}
$$

The electron power loss detection efficiency, $\eta_{\text {net }}$, is given by the convolution of the thick target spectrum and the camera response, normalized by the total electron energy flux,

$$
\eta_{n e t}\left(T_{e}\right)=\frac{\int_{0}^{\infty} I(E) R(E) d E}{\int_{0}^{\infty} V_{r}^{r} F(W) d W}
$$

where $F(W)$ is the particle flux of electrons with energy $W$ used to calculate $I(E)$ (see chapter II, section C). A plot of $\eta_{\text {net }}$ versus electron temperature is shown in the top of figure VII.C.1. 

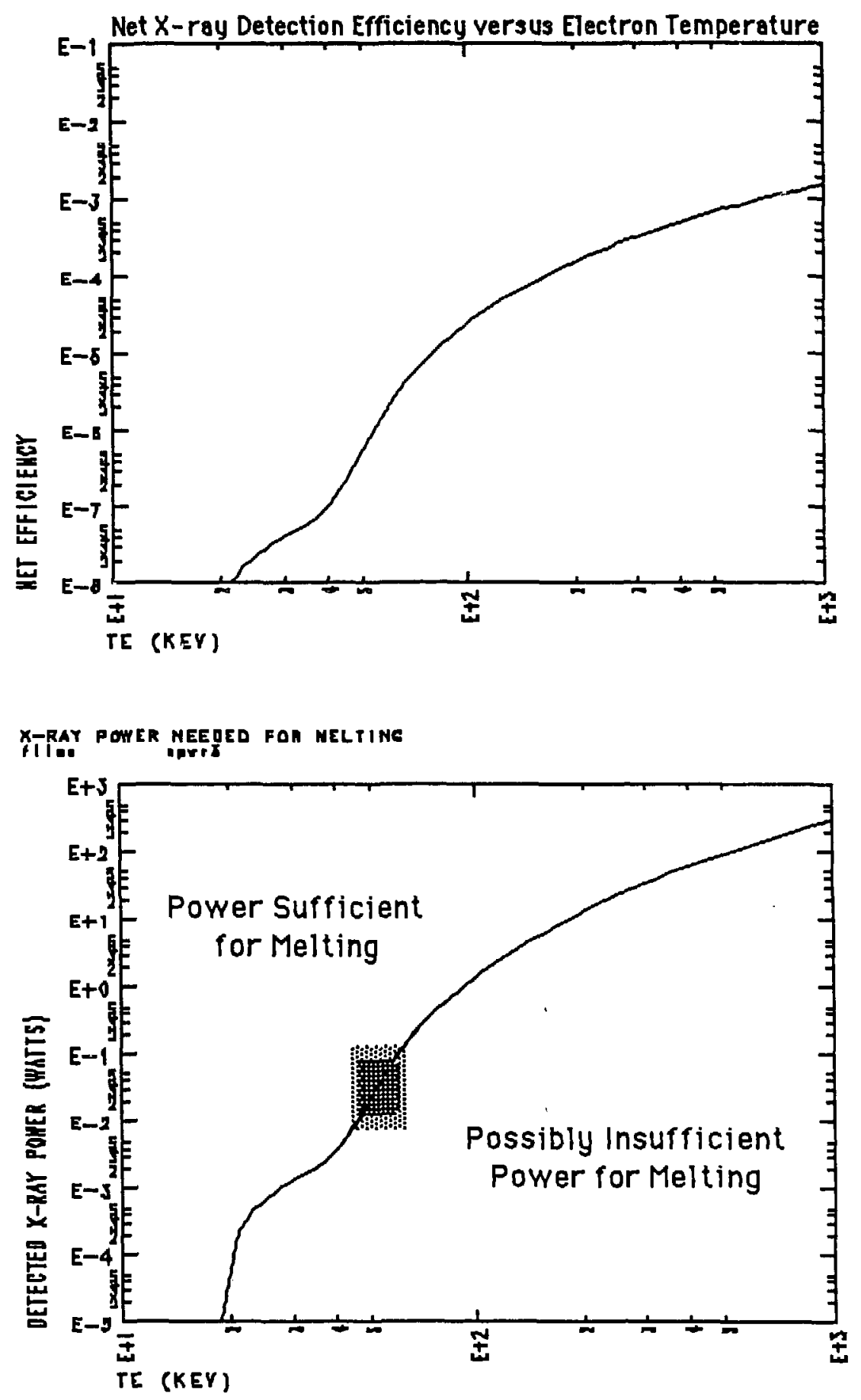

Figure VII.C.1. X-ray detection efficiency and detected flux necessary for DML shield melting. 
The product of the power necessary for melting, $P_{\text {melt }}$, and the net efficiency, $\eta_{n e t}$, provides an upper limit on the electron temperature. A plot of this curve is shown at the bottom of figure VII.C.1. Because the scrape off length increases with electron temperature, points to the right of the curve correspond to electron stream intensities insufficient to melt the shield. During the shot, the peak detected thick target $x$-ray power (with the $\mathrm{Pb}$ filter and $\mathrm{CsI}$ scintillator combination described above) was between $10^{-2}$ and $10^{-1} \mathrm{~W}$. If the electrons striking the shield can be described by a single temperature, it would have to be between 45 and $80 \mathrm{keV}$ to obtain melting, taking into account the uncertainty in absolute calibration of the camera. This temperature is between that of the warm (18-25 keV) and hot (100-400 keV) electron components, and suggests that electrons from both populations are being lost during the heating pulse.

Experimental measurements indicate that the hot $(100-400 \mathrm{keV})$ electrons can be lost radially. A comparison of the UPHP30 and SAI images indicates that there is a warm electron radial loss for both shots, but that there are hot electron losses only for the UPHP30 case. This is indicated by the fact that emission is detected through the 500 mil $\mathrm{Pb}$ filter for the former and not for the latter. It should also be noted that the level of energy stored in the hot electrons was typically much lower for the SAI case, which would mean that the production of very energetic electrons $\left(T_{e} \sim 250 \mathrm{keV}\right)$ was greatly reduced. As was the case with the hot electron losses during the magnet decay, these losses can be explained by a change in pitch angle due to a reduction of the local magnetic field. During the microwave heating 
pulse, as the stored energy in the hot electrons increases the vacuum magnetic field is depressed moving confined particles toward the loss boundary. Also, theoretical calculations suggest that if the microwave heating field is sufficiently large, the "strong heating" limit, ${ }^{8}$ strong velocity space diffusion will result. Radial electron losses due to this process would explain why the observed thick target emission is stronger on the north, where the heating field is stronger, as compared to the south. Magnetic field depression and strong rf velocity space diffusion may explain why mirror-trapped electrons are observed to be lost radially when the plasma diamagnetism is increasing during the microwave heating pulse.

\section{Conclusion}

In summary, the detected thick target emissions due to electrons striking two locations on the diamagnetic loop are the result of poor electron drifts at large radii. The drifts will intersect the loop if the electron $x$-axis intercept is sufficiently large and its pitch angle is sufficiently small. The fact that the iron shield is melted by these electrons indicates that on the order of $30 \mathrm{~kW}$ of power is being lost, which is a good fraction of the incident second harmonic power $(\sim 140 \mathrm{~kW})$. Also, the thick target emission becomes much stronger late in the microwave heating pulse, consistent with increased absorption at large radii. A reason for this increased absorption is given in the next chapter, which discusses measurements made of the volume emission. 


\section{Chapter VIII \\ Volume Emission}

\section{A. Introduction}

Measurements show that the radius of the plasma x-ray emissivity generally increases with stored energy per unit length, as measured by a diamagnetic loop. These results are summarized in figure VIII.A.1; each data point corresponds to a different shot. The nominal gaussian radius of the emissivity for shots with high stored energy values is $16 \mathrm{~cm}$. The radius of the mirror-trapped electrons which corresponds to this is $22 \mathrm{~cm}$, assuming that the emissivity is proportional to the square of the electron density (the ions have the same radial profile as the electrons). For a given microwave power and center cell density, the increase in stored energy can be attributed mainly to an increase in plasma volume rather than an increase in energy density. Also, for a given plasma fueling rate, the energy density increases with increasing second harmonic microwave power.

\section{B. Experimental Data}

Because the $x$-ray signal was weak and the stored energy increased rapidly with time, it was difficult to see variations in the plasma radius with stored energy by simply looking at different times during a shot. However, a series of shots, which used atypical timing signals, allowed variations to be seen. 


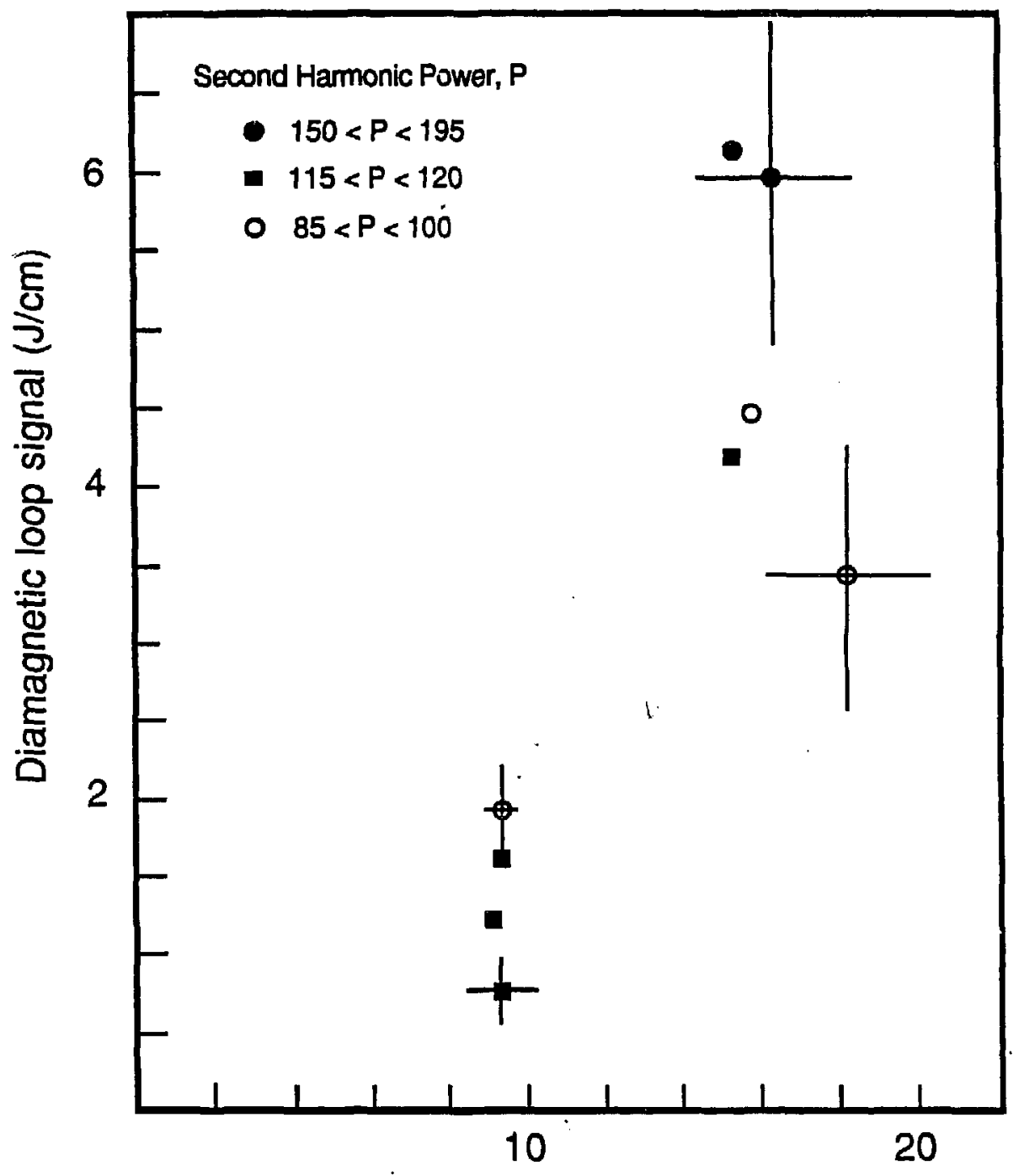

Gaussian radius of $x$-ray emissivity $(\mathrm{cm})$

Figure VIII.A.1. Variation of measured stored energy per unit length with the radius of the $x$-ray emissivity measured by the $x$-ray pinhole camera. 
Shown in figures VIII.B.1 and 2 are the west end cell diamagnetic loop and electron density signals for two such shots. The main difference between them which one would expect to affect the production of hot electrons is the duration of the second harmonic heating pulse. For shot 9, this pulse lasted only $20 \mathrm{msec}$ at the beginning of the shot. Note that the diamagnetism stays flat during the remainder of the shot while the plasma is being sustained by first harmonic heating only. Late in time the sloshing beams are turned on and the electron density rises sharply. This rise could be attributed to two things-improved ion confinement as the bulk ions are replaced by sloshing jons from the beams through charge exchange, or ionization of neutrals produced by the beam wall reflux. Impurities would have a smaller ionization energy than deuterium, hence the large increase in electron density. During this period of beam injection, an x-ray image was recorded. On shot 19 , the second harmonic heating pulse lasted $60 \mathrm{msec}$, and an $\mathrm{x}$-ray exposure was obtained from 60 to $77 \mathrm{msec}$, at a higher diamagnetism value than shot 9 .

A plot of the same radial intensity lineout for both shots is shown in figure VIII.B.3. Note that the intensity (which corresponds to a line averaged emissivity) measured for shot 19 is about twice as broad and high as shot 9. This would imply that the peak emissivity (at zero radius) is about the same for both shots; shot 19 simply has a larger plasma radius.

The radius of the plasma emissivity was determined by fitting the measured intensities to a model. Two models were used-a parabola and a gaussian. See figure VIII.B.4. Reduced $\chi^{2}$ values of order unity indicate a satis- 


\section{Mochine Timing}
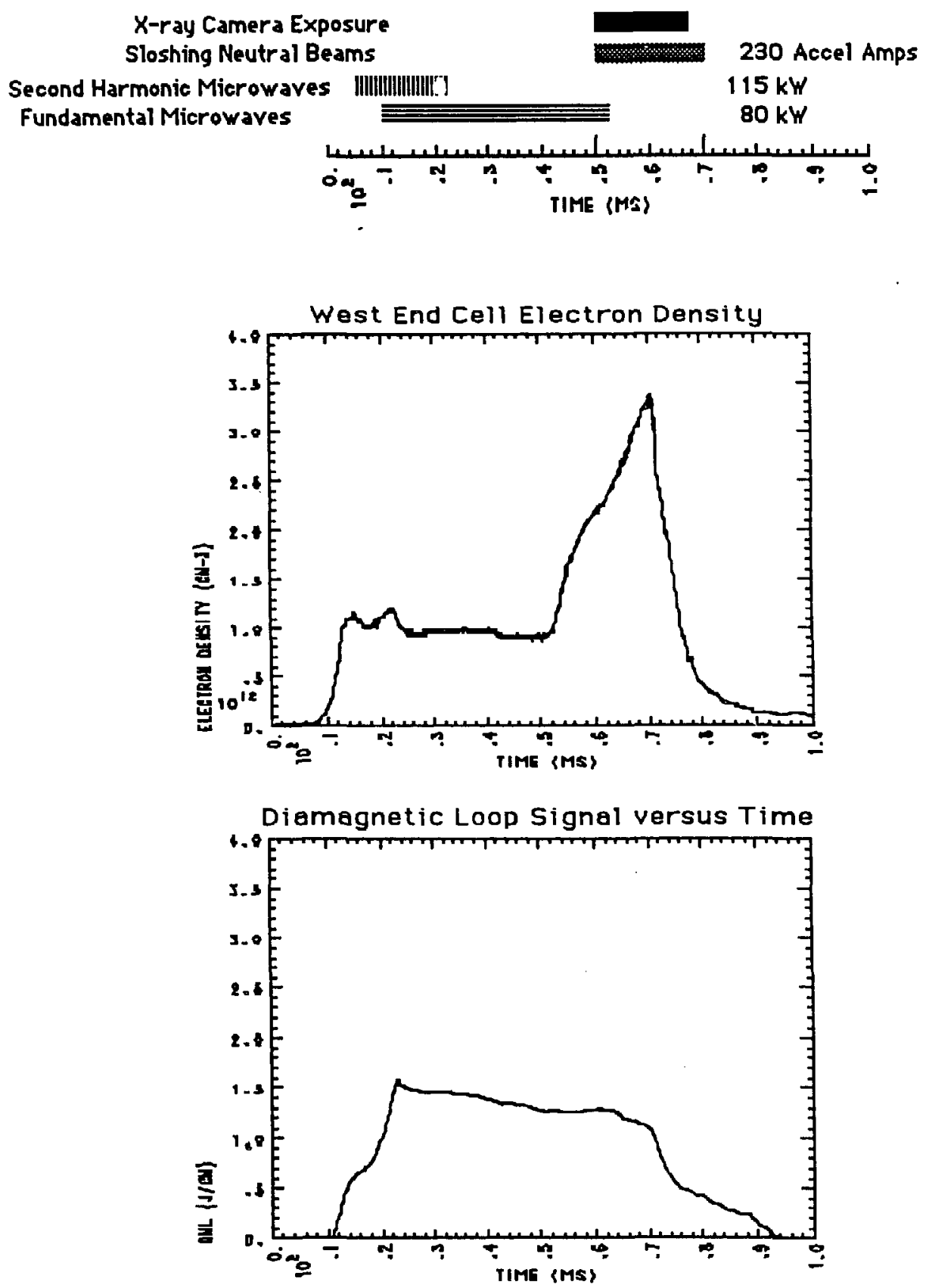

Figure VIII.B.1. Short second harmonic heating pulse-shot 9, 20 May 1985. 


\section{Machine Timing}

\section{$X-r a y$ Camera Exposure}

Sloshing Neutral Beams

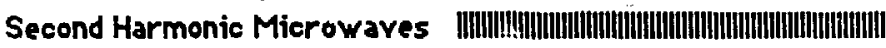
Fundamental Microwaves
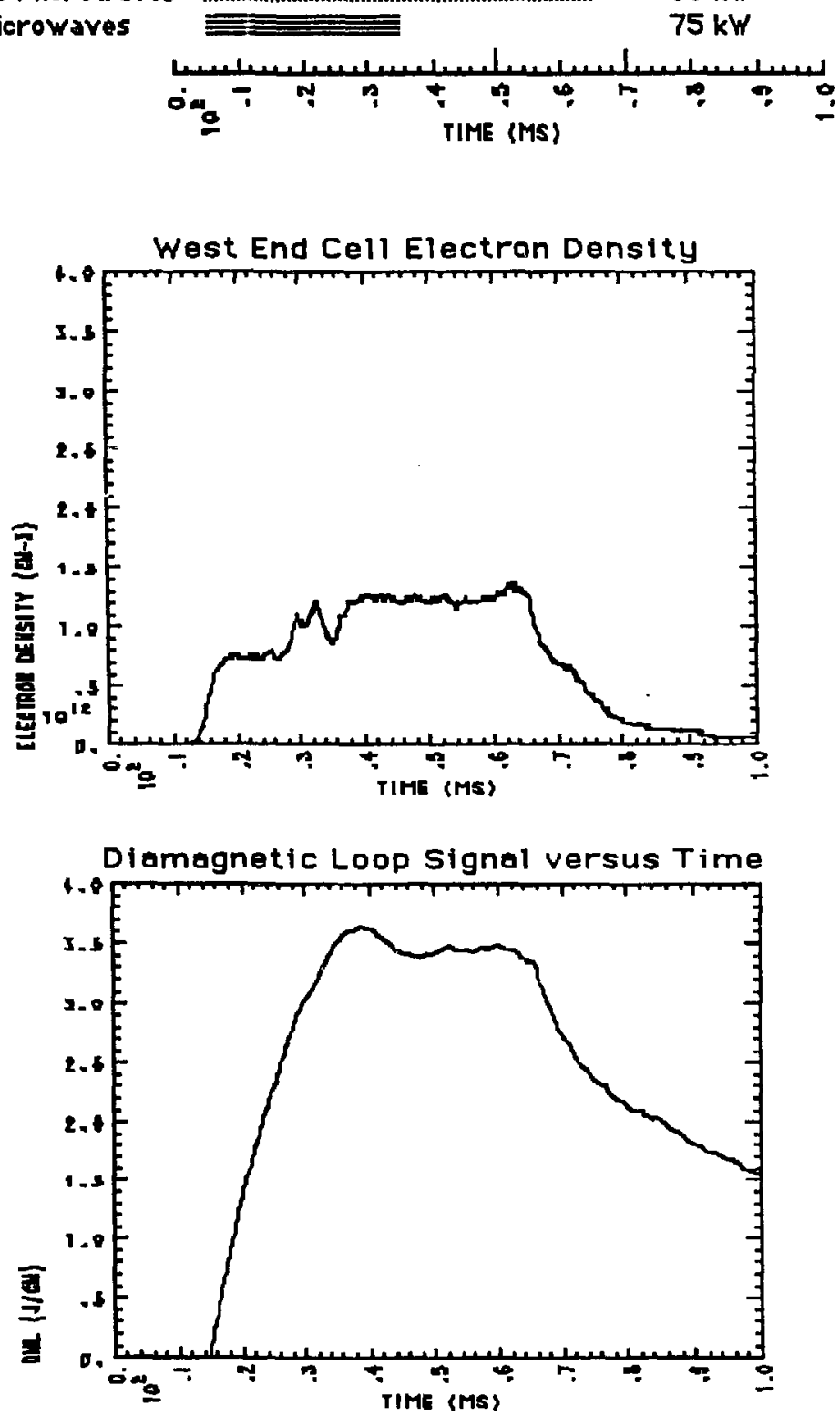

Figure VIII.B.2. Long second harmonic heating pulse-shot 19, 20 May 1985. 


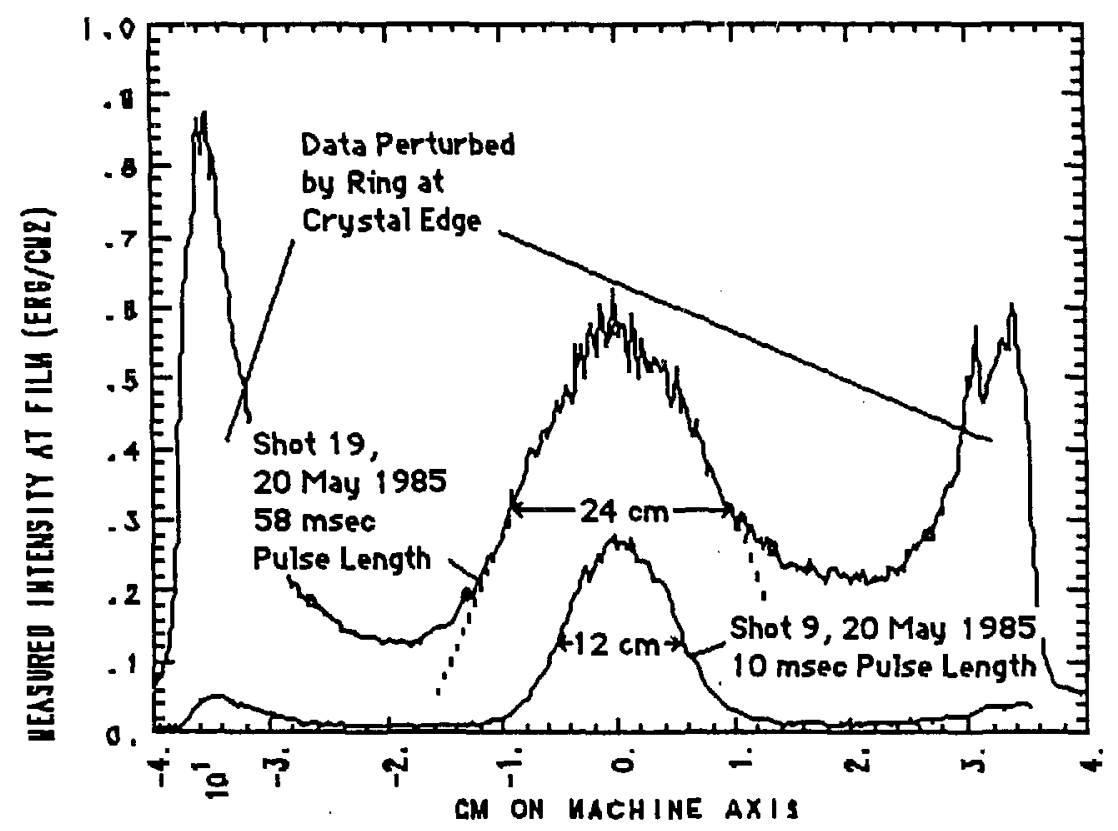

Figure VIII.B.3. Radius of $\mathrm{x}$-ray emissivity is larger for longer second harmonic pulse lengths. 
factory fit, while higher values indicate that the fit may not be appropriate to the data. ${ }^{1}$ The gaussian gave a better fit, with a reduced $\chi^{2}$ value of .9 . For the parabolic fit the same parameter was consistently around 1.2. Both models fit the data well, but a comparison of the $\chi^{2}$ values (for 36 degrees of freedom) indicates that the gaussian will provide a better fit about $15 \%$ more often. For this reason, the gaussian fit radii were used as a measure of the radial $\mathrm{x}$-ray emissivity distribution.

While unambiguous variation in the emissivity radius has been observed, no definite axial variations were measured. An example of this is shown in figure VIII.B.5. Different axial locations ( $z$ values of $526,530,533,536,552$, 555 , and 558), are indicated by different symbols. The experimental error for the intensity points shown is about $5 \%$. This lack of axial variation can be explained by the fact that the magnetic field varies by only a few percent over the x-ray camera's axial field of view. Thus very few of the mirror-trapped electrons will mirror in that region.

\section{Discussion}

It should be noted that energy losses due to bremsstrahlung emission are negligible. Typical $\mathrm{x}$-ray emissivities measured with the $\mathrm{x}$-ray camera are about $10 \mathrm{ergs} / \mathrm{cm}^{3}$-sec. Multiplying this emissivity by the duration of the microwave heating pulse, $\sim 50 \mathrm{msec}$, one obtains $.5 \mathrm{erg} / \mathrm{cm}^{3}$, while the stored energy density as calculated from the plasma diamagnetism is on the order of $10^{5} \mathrm{erg} / \mathrm{cm}^{3}$. 

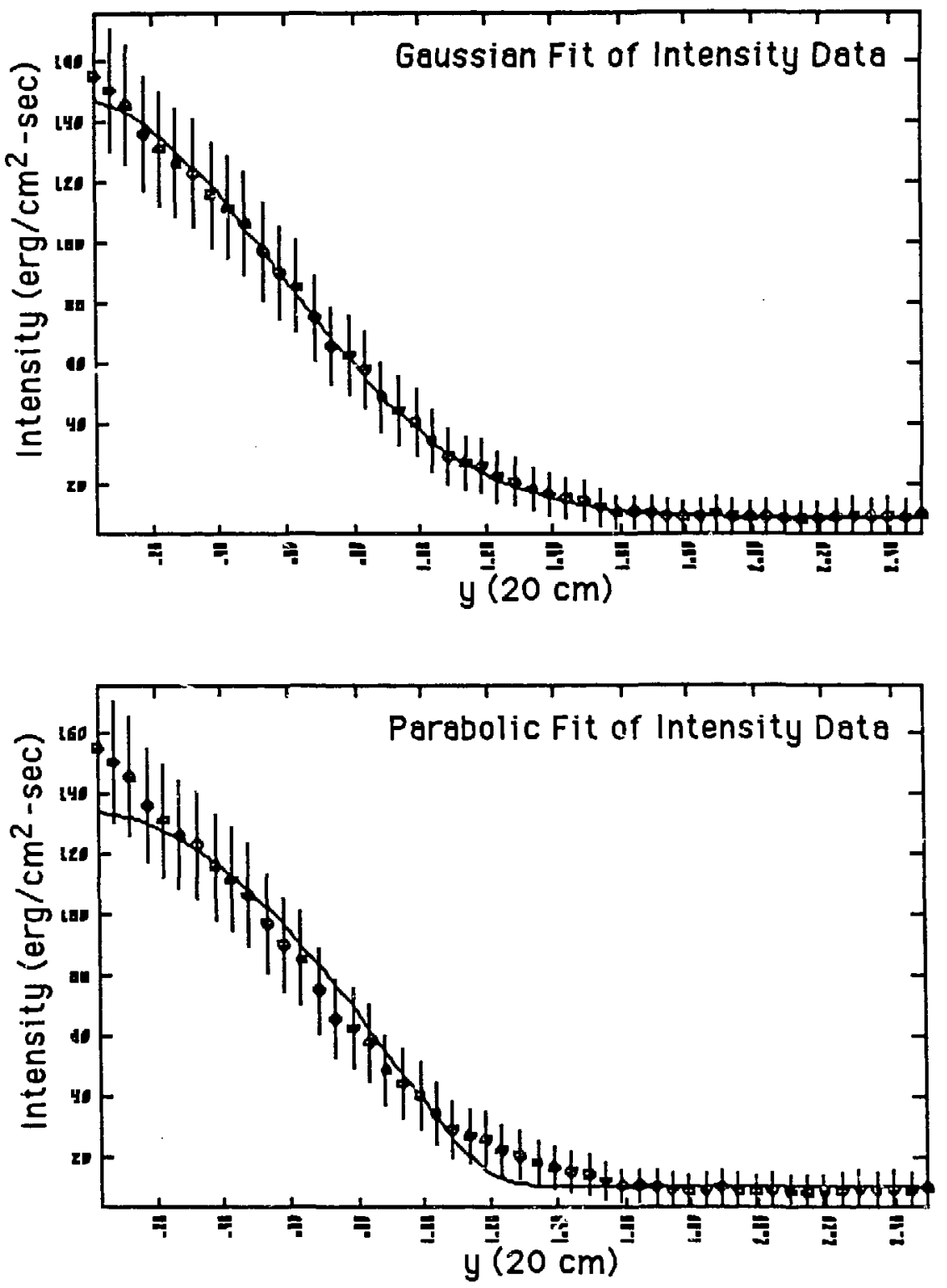

Figure VIII.B.4. Gaussian and parabolic fits of $x$-ray intensity data. 
RADIAL INTENSITY

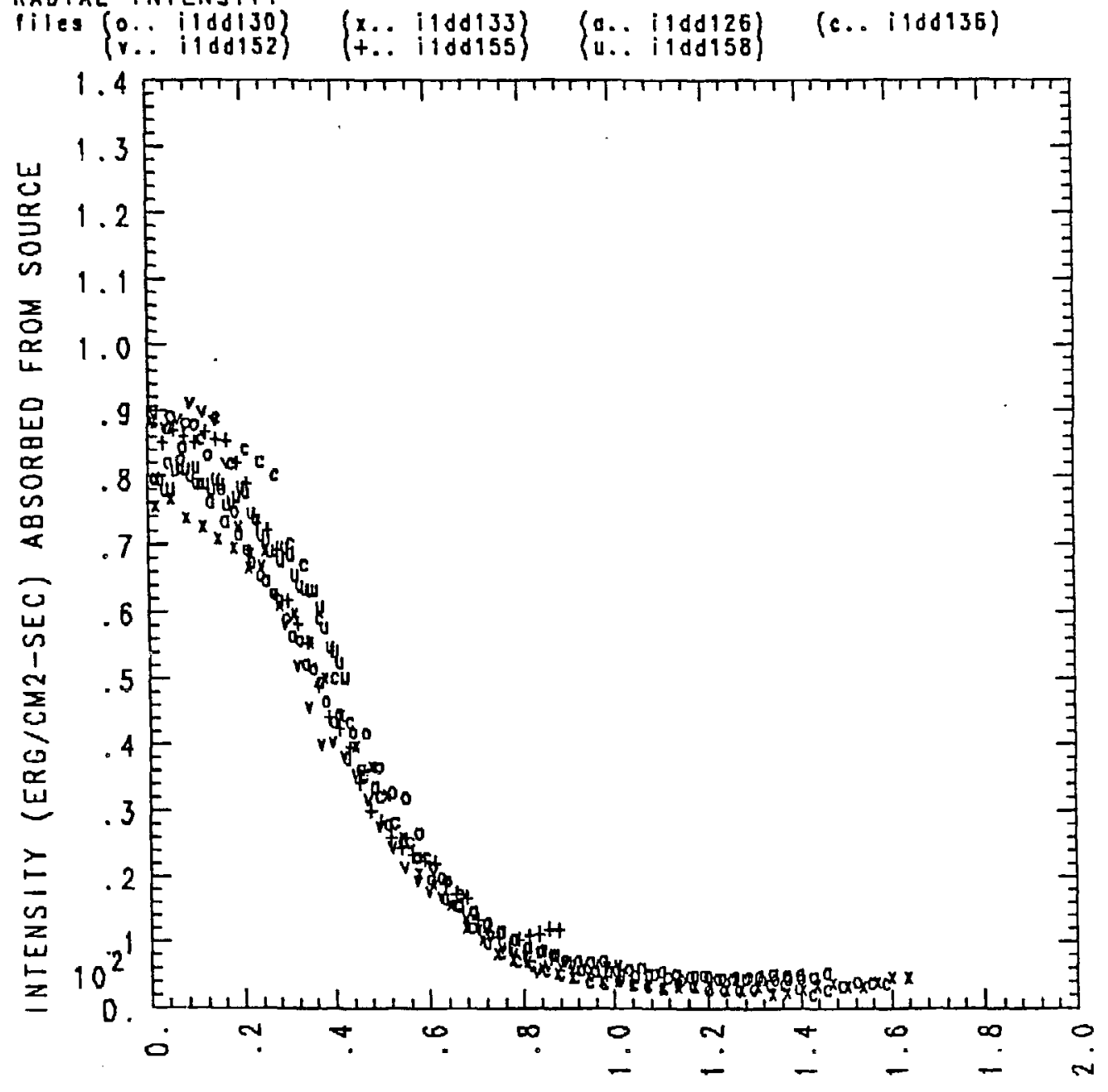

$R / 20 \mathrm{CM}$

Figure VIII.B.5. Radial intensity profiles at a seven axial locations-shot 8, 20 May 1985. Relative errors are similar to those shown in figure VIII.B.4. 
During the microwave heating pulse, the energy stored in the mirrortrapped electrons, as measured by the diamagnetic loop (DML), is seen to increase. The DML signal is proportional to the plasma pressure, integrated over the plasma cross-section. For an isostropic Maxwellian momentum distribution, the local pressure is equal to $n_{m}(r) T_{e}(r)$, and the integral (assuming azimuthal symmetry) is therefore given by

$$
D M L \propto 2 \pi \int_{0}^{r_{D M L}} n_{m}(r) T_{e}(r) r d r
$$

where $r_{D M L}$ is the DML radius, $n_{m}$ is the mirror-trapped electron density, and $T_{e}$ is the Maxwellian temperature. Describing the mirror-trapped electron density by a gaussian,

$$
n_{m}(r)=n_{m} \exp \left(-\frac{r^{2}}{r_{p}^{2}}\right)
$$

as suggested by the x-ray image data, and in the approximation that $T_{e}(r)$ is constant, the integration yields

$$
D M L \propto n_{m} T_{e} \pi r_{p}^{2}
$$

Thus the rise in stored energy can be explained by an increase in the energy density, $n_{m} T_{e}$, an increase in the plasma radius, $r_{p}$, or a combination of both. As discussed in section A, the plasma radius, as calculated from the $\mathrm{x}$-ray emissivity profile, generally grows as the stored energy increases, and this observed growth is sufficient to explain all, or a large fraction, of the detected rise in the DML.

An increase in plasma radius with stored energy can be explained by a depression of the vacuum magnetic field due to mirror-trapped electron $\beta$. $\beta$ is defined as the ratio of plasma pressure or energy density, $n_{m}(r) T_{e}$, to 
magnetic field pressure. As $\beta$ increases, the local magnetic field will decrease in accordance with the relationship

$$
B(r)=B_{0}(r) \sqrt{1-\beta(r)}
$$

where $B_{0}$ is the vacuum field. As shown in figure VIII.C.1, the second harmonic resonance will move out radially with the mirror-trapped electron radius, $r_{p}$, for a given peak energy density, $n_{m} T_{e}$. In the plot at the top of the page are shown energy density profiles corresponding to three different values of $r_{p}$. In the plot at the bottom both the vacuum and also the depressed magnetic pressure radial profiles, corresponding to the three $r_{p}$ values, are shown. Heating on the machine axis will be reduced once a finite $\beta$ has been achieved for two reasons-first there is no longer a cold plasma resonance there, and second the strong absorption at finite radius will attenuate the power before it can reach the axis.

A schematic describing the way in which the mirror-trapped electron radius grows is given in figure VIII.C.2. Early in a shot, the microwaves are absorbed at the location of the vacuum resonance. As the power is absorbed and the stored energy increases, the loca! magnetic field is depressed and the resonance shifts to a new location. Because the magnetic field configuration in the plug region is minimum $|\mathbf{B}|$, the new resonance will always be located at a larger radius than the previous one. The microwaves are absorbed at the new location until the local field is again depressed, and the resonance again shifts. This is the mechanism by which the mirror-trapped electron radius, $r_{p}$, increases during the heating pulse. 

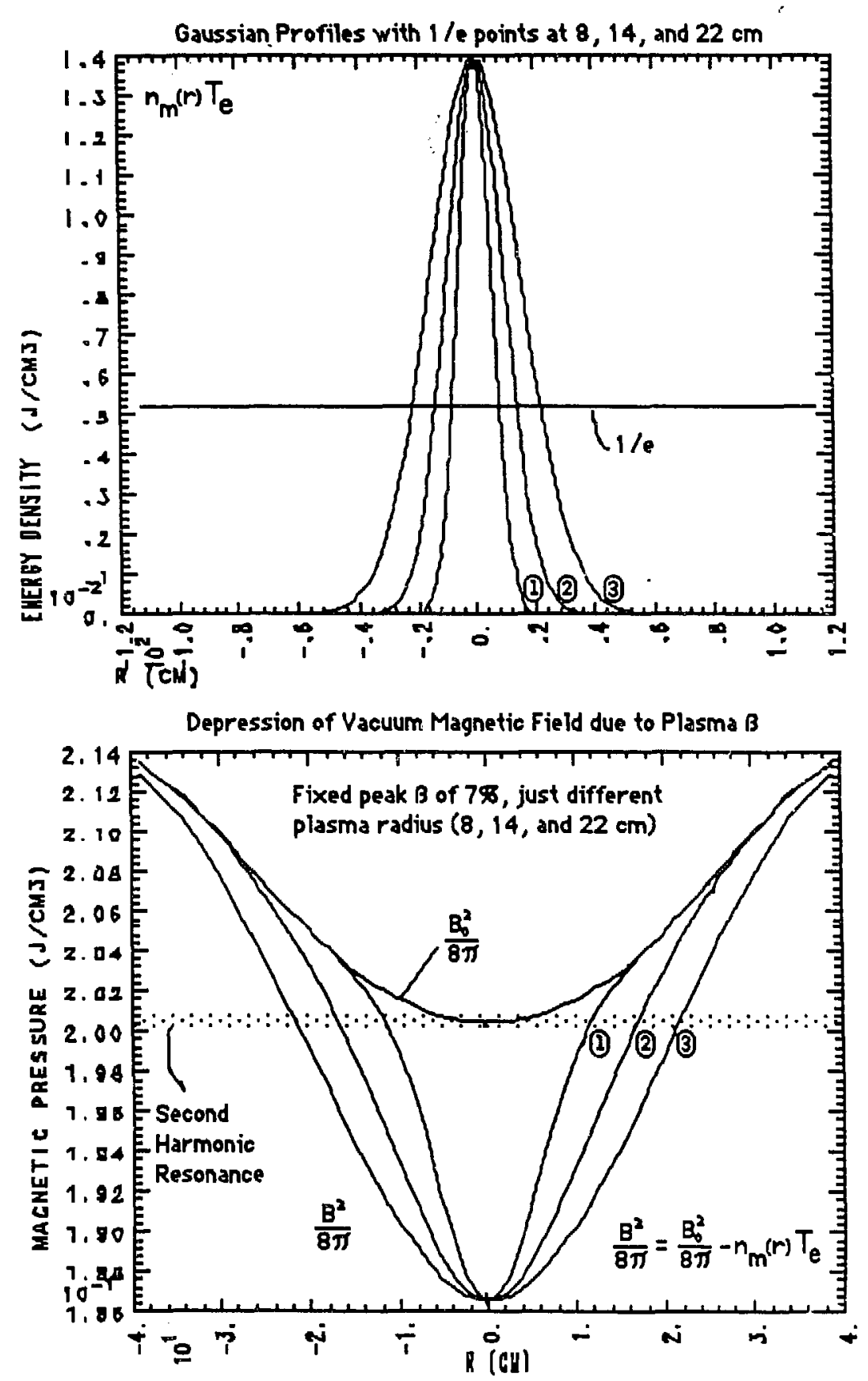

Figure VIII.C.1. For fixed peak B, the second harmonic resonance moves out radially with the mirror-trapped electron radius. 


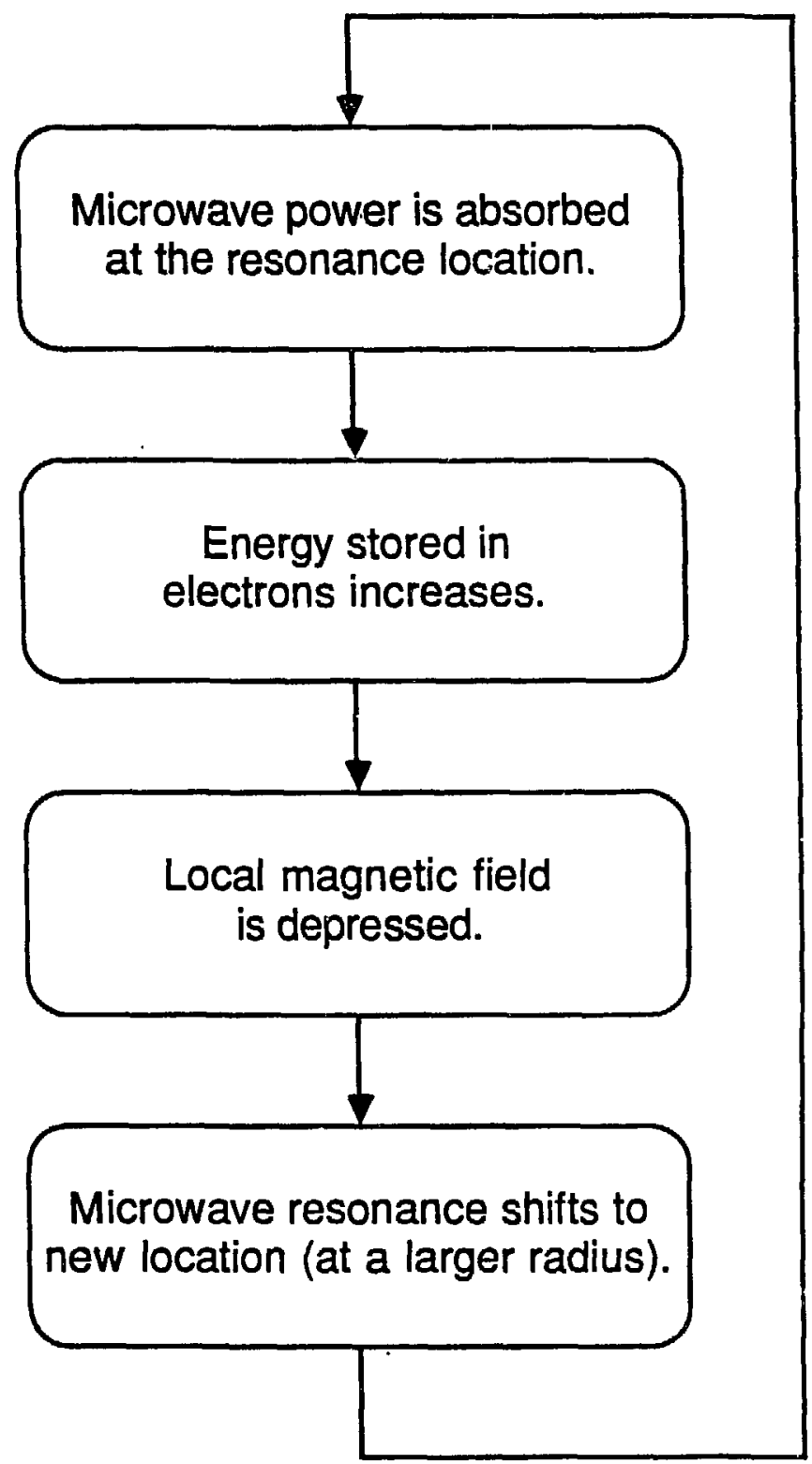

Figure VIII.C.2. Schematic describing how the radius of the mirror-trapped electrons increases during the microwave heating pulse. 
If the peak electron energy density, $n_{m} T_{e}$, remains fixed during a shot, the square root of the diamagnetic loop signal will be directly proportional to the mirror-trapped electron radius. In this approximation, the DML signal during the camera exposure can be normalized to the measured $\mathrm{x}$-ray emissivity radius to obtain a measure of the mirror trapped electron sadius as a function of time. Two examples of the electron radius versus time calculated in this manner are shown in figure VIII.C.3. The diamagnetic loop signal was normalized to the $\mathrm{x}$-ray emissivity radius at the two points shown. The curve for shot 21 was shifted back $5 \mathrm{msec}$ in time, so that the points where the initial rapid rise occurs will coincide. The second harmonic power level was identical for the two shots, $115 \mathrm{~kW}$, but the pulse lengths were different $-17 \mathrm{msec}$ for shot 10 , and $54 \mathrm{msec}$ for shot 21 . The radius increases rapidly in time, reaching $20 \mathrm{~cm}$ in less than $18 \mathrm{msec}$ for shot 21 . During the time that the second harmonic heating system is on for both shots the agreement in the two curves is good. This result is consistent with the model for the microwave absorption described above.

Other diagnostic measurements were examined to see if they were consistent with this picture, namely that the energy density of the plasma was increasing on a much slower time scale than the radial growth, and that for a given feed of cold electrons the peak $\beta$ should increase with increased microwave power. Two sets of data were examined, one in which a limiter was moved in and out radially and another which had variations in second harmonic power for the same gas fueling. 


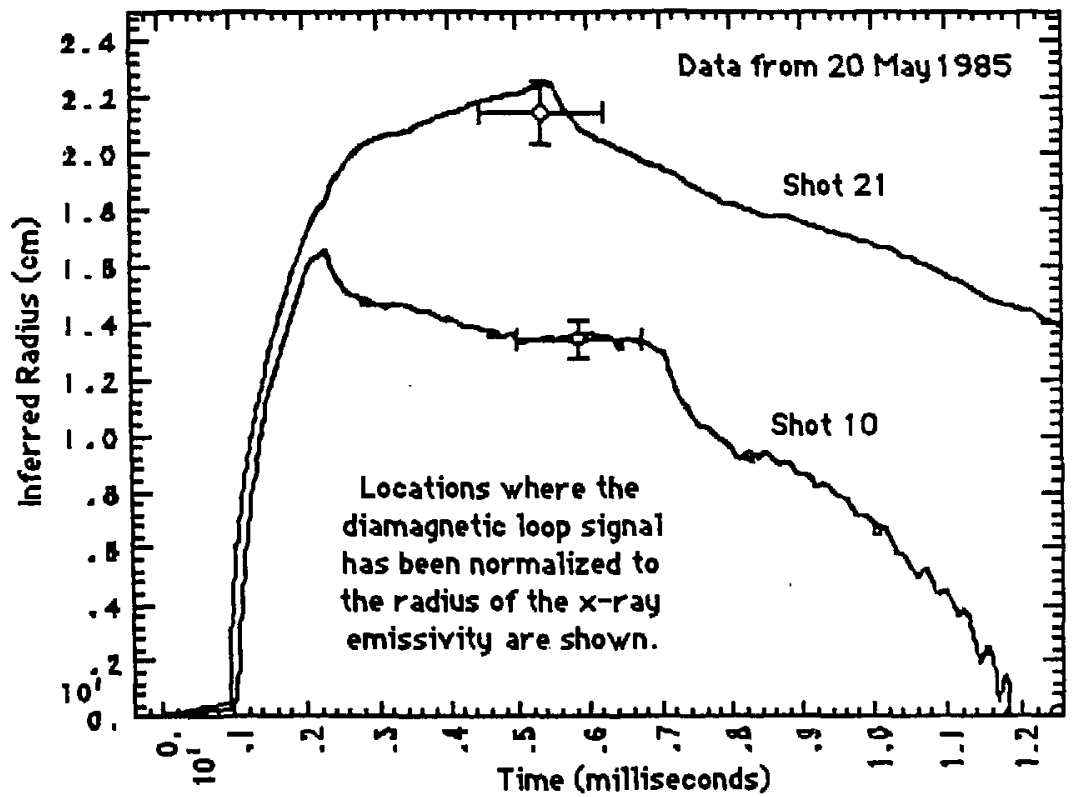

Figure VIII.C.3. Variation of mirror-trapped electron radius obtained from the diamagnetic loop signal normalized to the measured $\mathrm{x}$-ray emissivity radius. Fixed peak $B$ approximation. 
Before the installation of the $\mathrm{x}$-ray pinhole camera, a series of measurements were made to try and determine the mirror-trapped electron radius by inserting a bumper limiter into the plasma, and monitoring the variations in plasma diamagnetism and electron cyclotron emission. ${ }^{2}$ If the mirror-trapped electron radius remained constant during heating, or ' ' would expect the diamagnetism rate of rise to vary with limiter location. But, if the variation in mirror-trapped electron diamagnetism was indeed dominantly the result of a variation in plasma radius, one would see the same rate of rise early in the shot, when the plasma radius is small, followed by a flattening out or saturation once the limiter location has been reached. These two scenarios are depicted in figure VIII.C.4.

The time evolution actually measured supports the time varying radius hypothesis. A plot of the plasma diamagnetism in the west end cell is shown in figure VIII.C.5 for a number of bumper limiter locations. Shots shown are those which had the most similar electron density traces, both in the center cell and the west end cell. The initial rapid rise in the plasma diamagnetism can be attributed to the heating and trapping of cold electrons, while the continued slower rise after the break in the signal is due to absorption by trapped electrons that are being heated up to higher energies.

An evaluation of how the stored energy varied with microwave power was made from the $60 \mathrm{GHz}$ perpendicular electron cyclotron emission (PECE) and the diamagnetic loop (DML) signals. Because the PECE horn has a finite radial field of view, call it $r_{h}$, its signal depends on whether the gaussian 
- If the mirror-trapped electron radius is constant during heating, the heating rate should decrease as the limiter is inserted, reducing the volume.

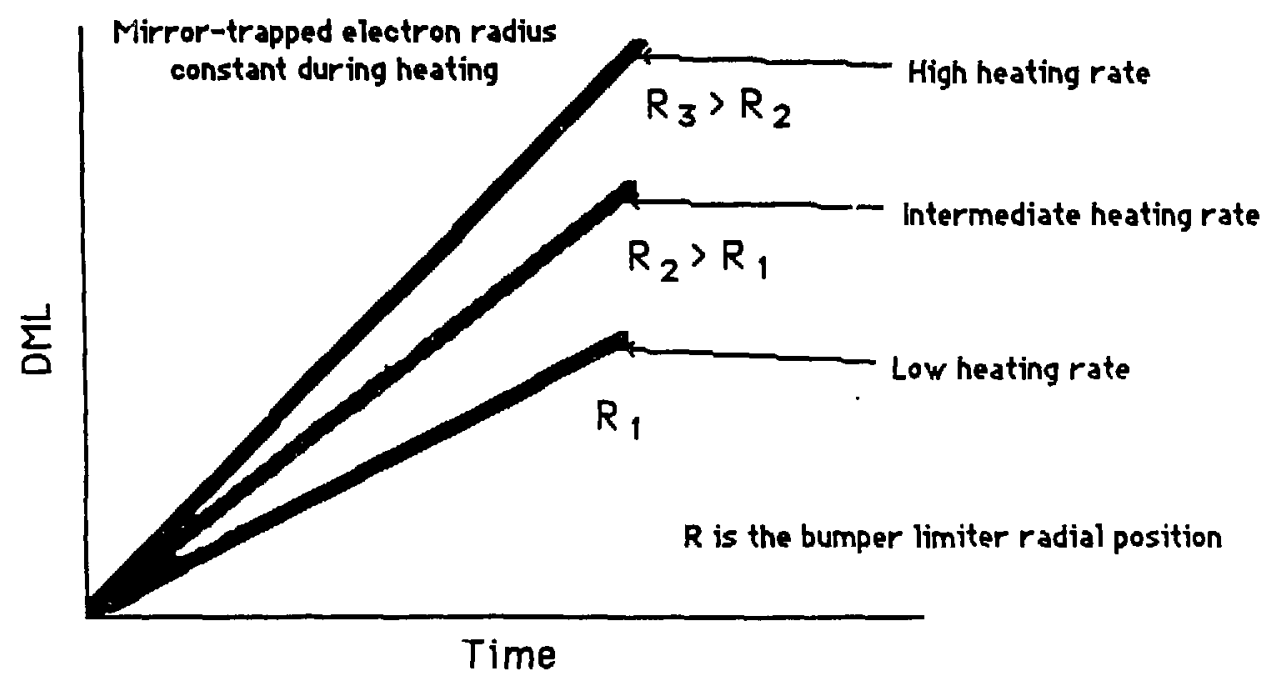

- If the radius does vary in time, and the rise of the DML is mainly due to an increase in plasma radius, the DML signal should increase until it comes in contact with the limiter.

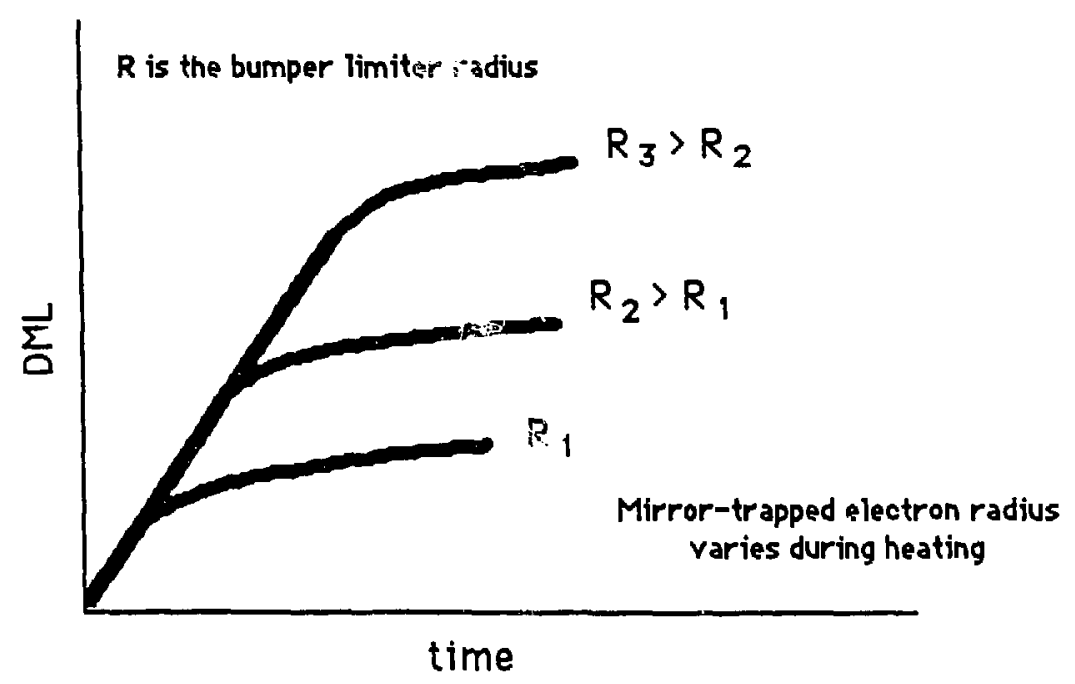

Figure VIII.C.4. Variation of plasma diamagnetism and heating rate with bumper limiter location. 


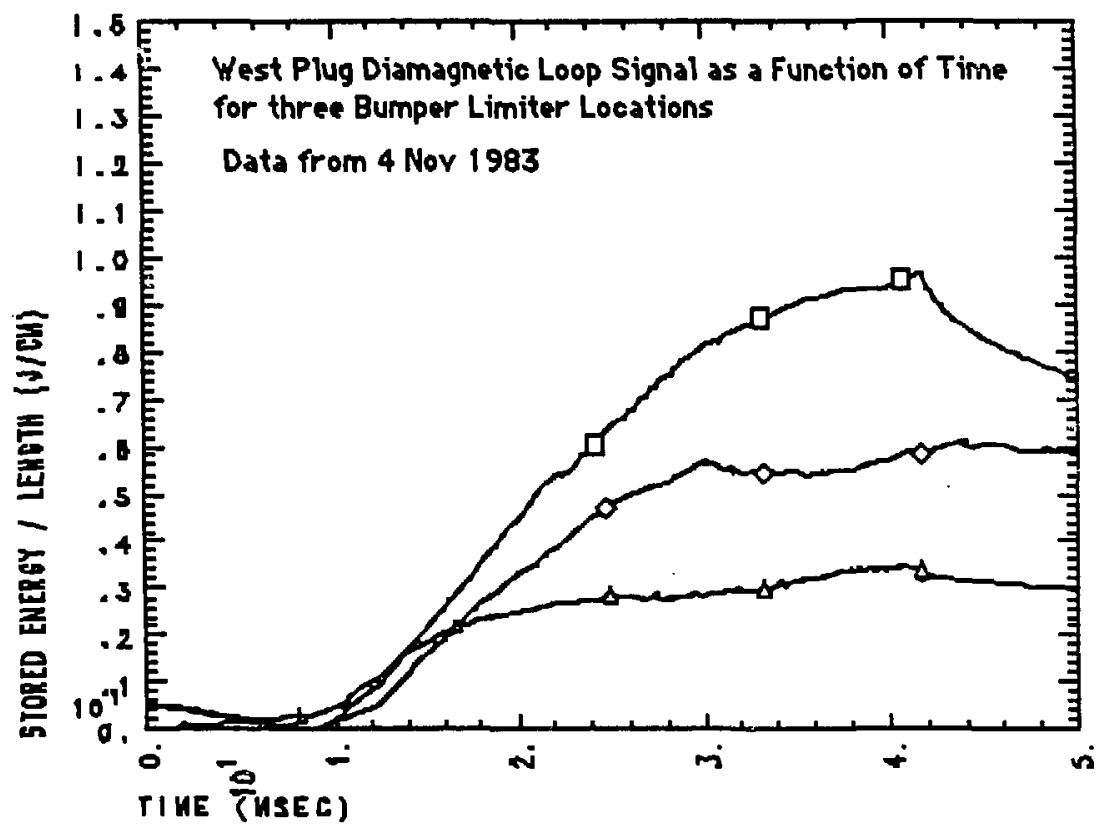

\begin{tabular}{|c|c|c|c|}
\hline & Shot Number & $\begin{array}{l}\text { Limiter Location } \\
\text { Actual }\end{array}$ & $\begin{array}{l}\text { Limiter Location } \\
\text { Mapped to Plug Midplane }\end{array}$ \\
\hline [] & 6 & Withdrawn & Withdrawn \\
\hline 0 & 19 & $26.2 \mathrm{~cm}$ & $19.7 \mathrm{~cm}$ \\
\hline$\Delta$ & 29 & $21.2 \mathrm{~cm}$ & $15.0 \mathrm{~cm}$ \\
\hline
\end{tabular}

Figure VIII.C.5. Change in diamagnetic loop signal as bumper limiter is inserted. 
plasma radius, $\tau_{p}$, is large or small in comparison to the horn field of view. The two limiting cases are shown below.

$$
P E C E\left(\mathrm{~J} / \mathrm{cm}^{2}\right)= \begin{cases}C_{1} n_{m} f\left(T_{e}\right) r_{p}^{2}, & \text { if } r_{p} \ll r_{h} \\ C_{1} n_{m} f\left(T_{e}\right) r_{h} r_{p}, & \text { if } r_{p} \gg r_{h}\end{cases}
$$

where $C_{1}$ is a constant, $n_{m}$ is the peak mirror-trapped electron density, and $f\left(T_{\mathrm{e}}\right)$ is the temperature dependence of the $60 \mathrm{GHz}$ PECE signal. Basically, when the plasma radius is large enough, the PECE is a line-averaged measurement.

The diamagnetic loop, however, is an area averaged measurement in the limit that the plasma has sufficient axial length so that fringing fields are unimportant. In the approximation that the temperature is uniform, the DML signal in the west end cell is given by

$$
D M L(\mathrm{~J} / \mathrm{cm})=C_{2} n_{m} T_{e} \pi r_{p}^{2}
$$

Where $C_{2}$ is a constant that depends on the magnetic field where the measurement is being made.

This means that by taking the square of the PECE signal and dividing it by the DML, one can obtain a measure of the peak energy density in the limit that $r_{p} \gg r_{h}$. Defining

$$
g\left(n_{m}, T_{e}, \tau_{p}\right)=\frac{P E C E^{2}}{D M L}\left(\mathrm{~J} / \mathrm{cm}^{3}\right)
$$

one has in the two limits for $\tau_{p}$

$$
g\left(n_{m}, T_{e}, r_{p}\right)= \begin{cases}C_{3} n_{m} \frac{f\left(T_{1}\right)^{2}}{T_{m}} r_{p}^{2}, & \text { if } r_{p} \ll r_{h} ; \\ C_{3} n_{m} \frac{f\left(T_{1}\right)^{2}}{T_{1}} r_{h}^{2}, & \text { if } r_{p} \gg r_{h}\end{cases}
$$


A plot of the expected behavior of $g$ versus DML is shown in figure VIII.C.6, in the limit that the plasma radius is constant and also the limit that the plasma radius is increasing more rapidly than anything else. For the second case, initially the slope should be linear, since both variables increase as $r_{p}^{2}$, but as $r_{p}$ approaches and begins to exceed $r_{h}, g$ should flatten out. Shown in figure VIII.C.7 is a plot $g$ versus DML for a plasma shot. The break in $g$ after it has saturated is correlated with a break in the DML. This break which corresponds to a change in the microwave heating rate, suggests that the nature of the heating has changed. The abrupt increase in $g$ may indicate that instead of dominantly heating the bulk electrons to warm temperatures, one is now mainly running the warm electrons up in energy.

A plot of $g$ versus $D M L$ should have a slope that depends only on electron temperature, for small $D M L$ values (corresponding to the limit $\left.r_{p} \ll r_{h}\right)$. The slope of the curve, $S$, is given by

$$
S=\frac{\Delta g}{\Delta D M L}=\frac{C_{3}}{C_{2} \pi}\left[\frac{f\left(T_{e}\right)}{T_{e}}\right]^{2}
$$

Two $g(\mathrm{DML})$ curves, plotted with logarithmic scales, are shown in figure VIII.C.8. On a plot scaled in this manner, a difference in slope between two curves is indicated by a constant displacement. The main difference between the shot with steep as opposed to shallow slope is the second harmonic heating power-190 kW for the steep and 110 for the shallow. The electron temperature, $T_{e}$, as measured by the high energy $\mathrm{x}$-ray spectrometer system (averaged over the heating pulse) was indeed higher for the shot with the larger initial slope: 190 versus $140 \mathrm{keV}$. This is consistent with the picture 

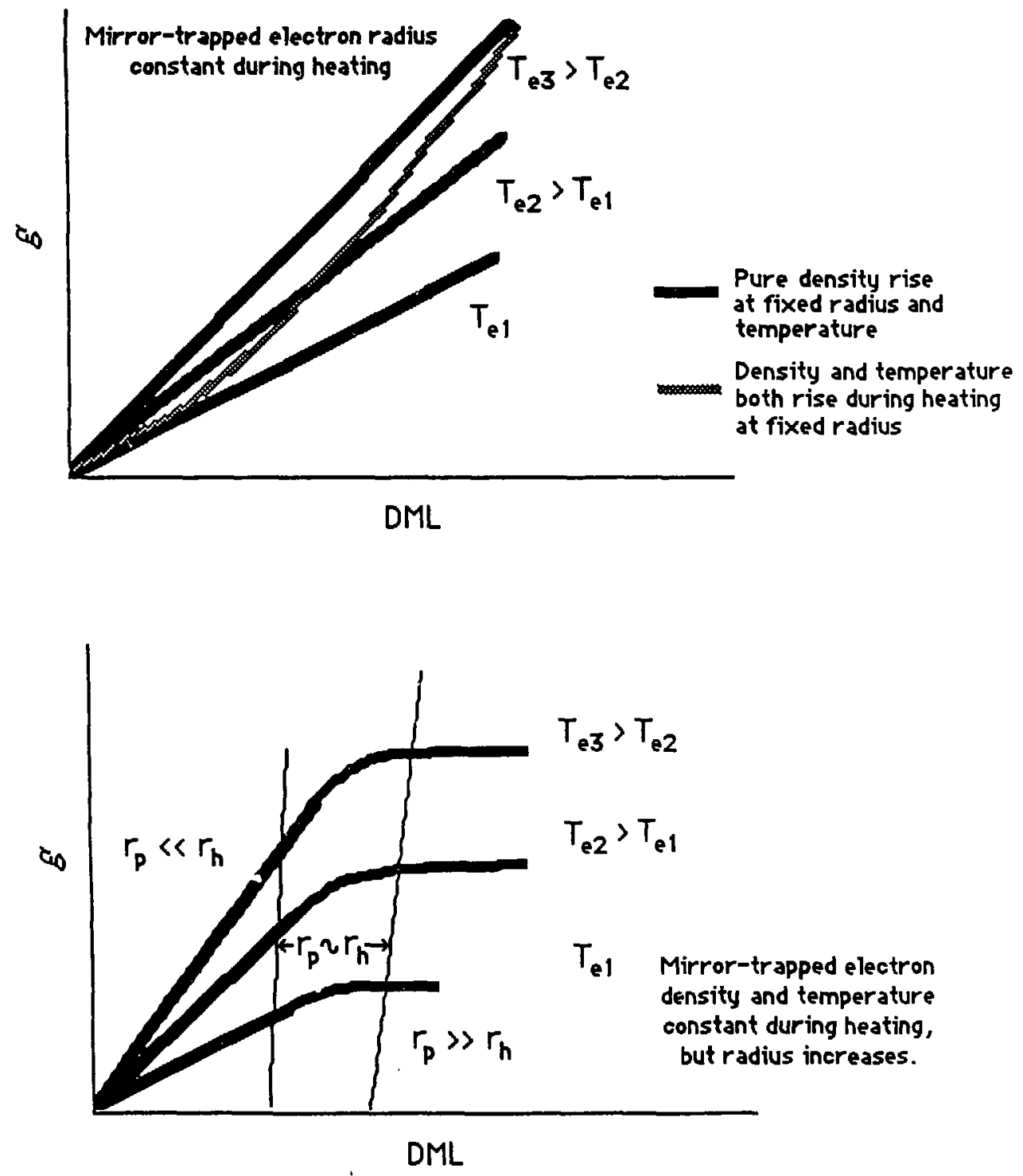

Figure VIII.C.6. Variation of $\boldsymbol{g}$ with Plasma Diamagnetism. 


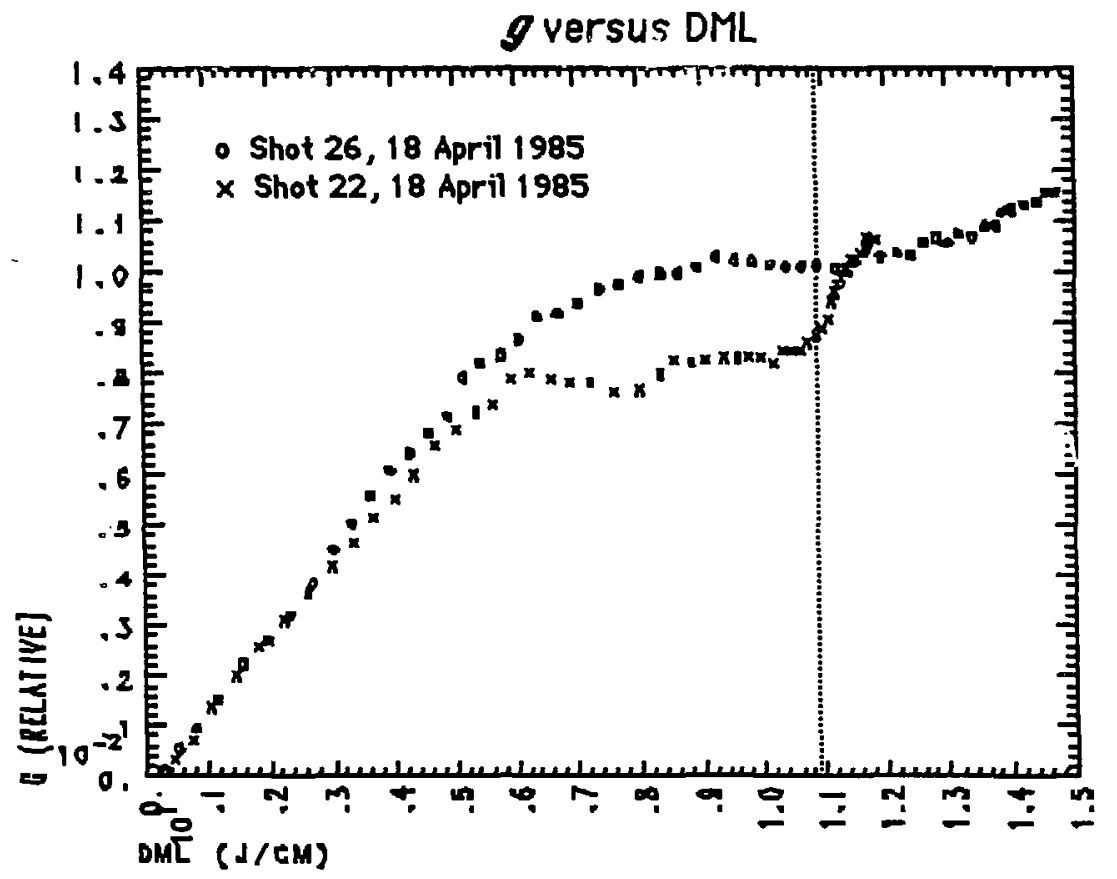

DML versus Time

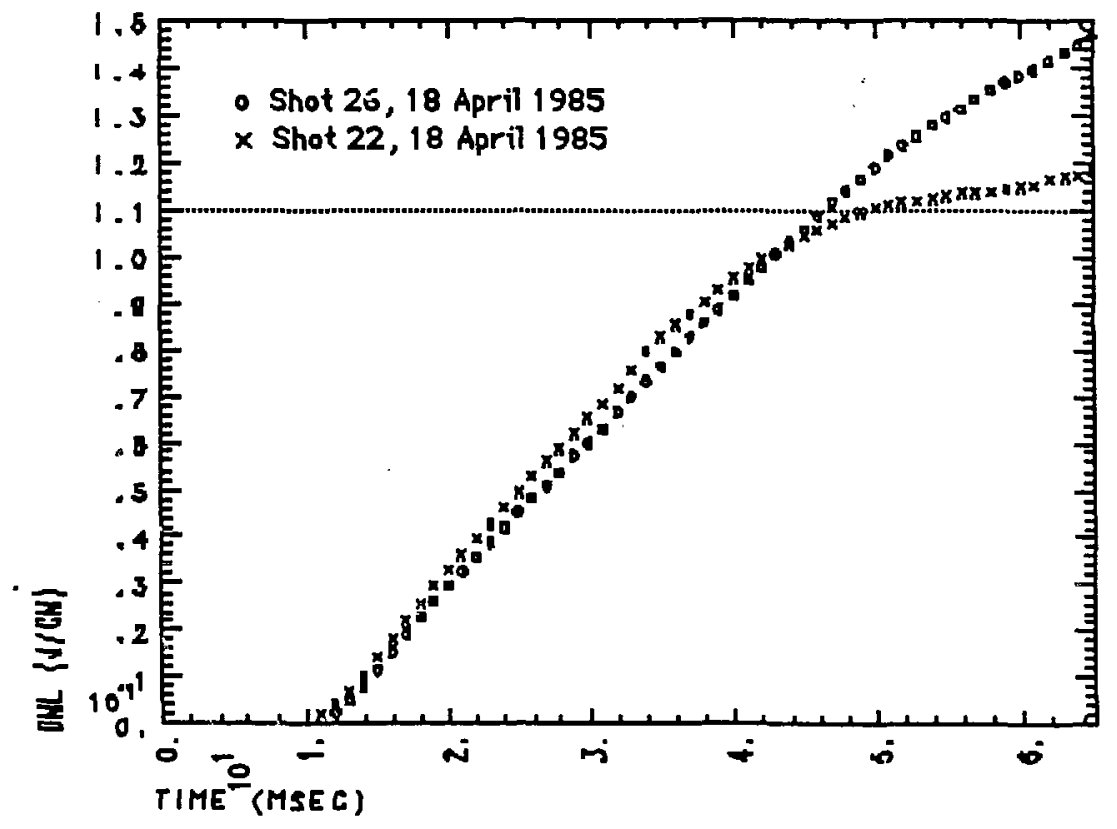

Figure VIII.C.7. Experimentally measured variation of $g$ with DML. 
that a higher peak energy density is achievable by going to higher microwave power levels. Unfortunately, not all of the increase in energy density is due to an increase in the number of trapped electrons. This is because the measured temperature of those electrons has also increased.

The microwave power in the end cell not only traps electrons in the plugs, it also serves as the power source for gas ionization and therefore can be used to start up the plasma initially. For this reason one might expect an increase in the mirror-trapped electron radius in the plug to affect the plasma radial profile in the center cell. Vacuum and neutron measurements indicate that this is the case. During the second harmonic heating pulse a pressure reduction is measured by a gauge located in the center cell. This is attributed to an expansion of the plasma radius until it reaches the gasbox limiters, which reduces the leakage of neutral $\mathrm{D}_{2}$ from the gas box to the gauge. ${ }^{3}$ Time-resolved neutron yield measurements are made near the midplane of the center cell using a collimated detector. These measurements indicate that when the end cell microwave heating is applied, center cell diamagnetism increases while the neutron yield is reduced. Because the yield is proportional to the ion density squared, this observation is consistent with a broadening of the ion radial profile in the center cell. ${ }^{4}$ These diagnostic measurements in the center cell substantiate the hypothesis that the plasma radius in the plug increases during the second harmonic heating pulse.

\section{Conclusion}

In this saction it has been shown that increases in stored energy are 


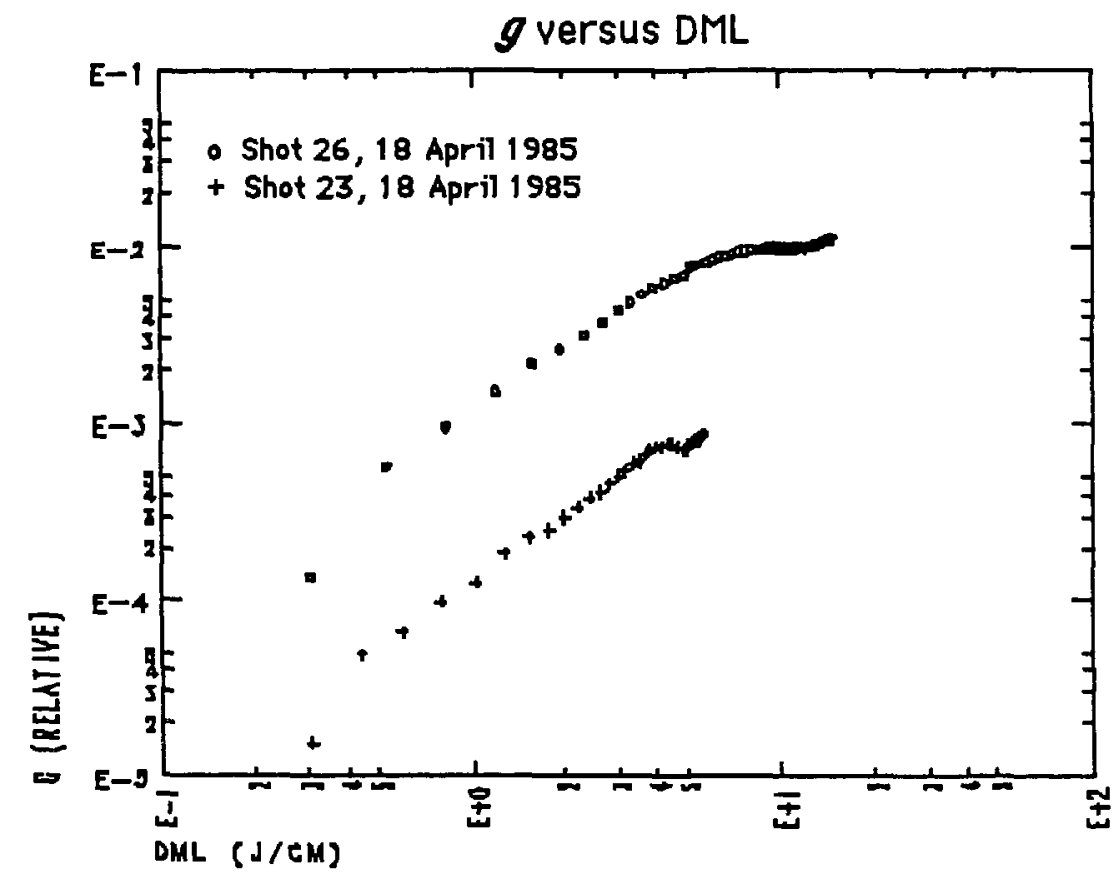

Figure VIII.C.8. Experimentally measured variation of $g$ with second harmonic power. Shot 23 had $90 \mathrm{~kW}$ of power, while 26 had 175. 
correlated with an increase in the mirror-trapped electron radius as inferred from the observed x-ray emissivity profiles. This increase in radius can be explained by a shift in the cold microwave absorption location, due to finite electron $\beta$. It has also been shown that the other mirror-trapped electron diagnostic signals, namely the DML and PECE are consistent with this picture. The way in the which the DML saturates when a bumper limiter is inserted is consistent with a radial growth of the mirror-trapped electrons, as is the variation of $g(\mathrm{DML})$. The level at which $g$ saturates also increases with absorbed second harmonic input power, as one would expect from the model. In addition, neutral pressure and neutron measurements in the center cell provide further evidence that the plasma radius is increasing with the stored energy. 


\section{Chapter IX}

\section{Correlations}

\section{A. Introduction}

In the previous section it was shown that the mirror-trapped electron radius generally increases as the stored energy in the plasma increases. This increase can be attributed to a shift in the cold plasma resonance due to finite plasma $\beta$. Because the plasma shape is evolving and the region where the microwave power is being absorbed is changing as the stored enlergy increases, the heating efficiency should also vary as the stored energy increases. In this section, evidence for this dependence will be shown, and its effects on machine performance discussed.

\section{B. Previous Analysis}

Theoretical calculations indicate that there should be an optimum cold electron feed which will produce the highest heating efficiency. ${ }^{1}$ In the absence of a thermal barrier, the cold electron feed is simply the fraction of the center cell electrons which are not mirror-trapped. For this reason, the heating efficiency should vary as a function of the center cell density.

Experiments have been done to test this hypothesis. ${ }^{2}$ The center cell density was varied by changing the gas feed; more gas typically produces a higher density plasma. A plot of heating efficiency versus center cell density is shown in figure IX.B.1. The circles correspond to data points early in time 
during a shot, and the squares to late ones. No correlation with the stored energy was made.

The conclusion from this analysis is that the heating efficiency is a strong function of the center cell density, dropping rapidly above a center cell density of about $1 \times 10^{12} \mathrm{~cm}^{-3}$. The theoretical calculations referenced above indicate that the trapping rate (the rate at which electrons flowing out of the center cell are heated to the point that they join the mirror-trapped population) could be improved by going to higher power levels. By doing this the heating rate, or rate of "rf diffusion," should be enhanced compared to the collisional scattering rate into the mirror loss cone. The analysis that follows, however, indicates that controlling the location where the microwaves are absorbed is more important to machine performance than increasing the microwave power level.

\section{Present Analysis}

For this analysis, the dependence of the heating rate on both center cell density and end cell diamagnetism was evaluated. To see a correlation between the heating efficiency and the center cell density, a finite range of stored energy values must be examined. The heating efficiency was found by differentiating the diamagnetic loop signal, multiplying by an estimate of the mirror-trapped electron length, $120 \mathrm{~cm}$, and dividing by the second harmonic heating power, $140 \mathrm{~kW}$. The data is from a series of shots taken on 1 April, 1985. There was no neutral beam injection, so a thermal barrier should not have formed. The microwave powers were 100 and $140 \mathrm{~kW}$ for 


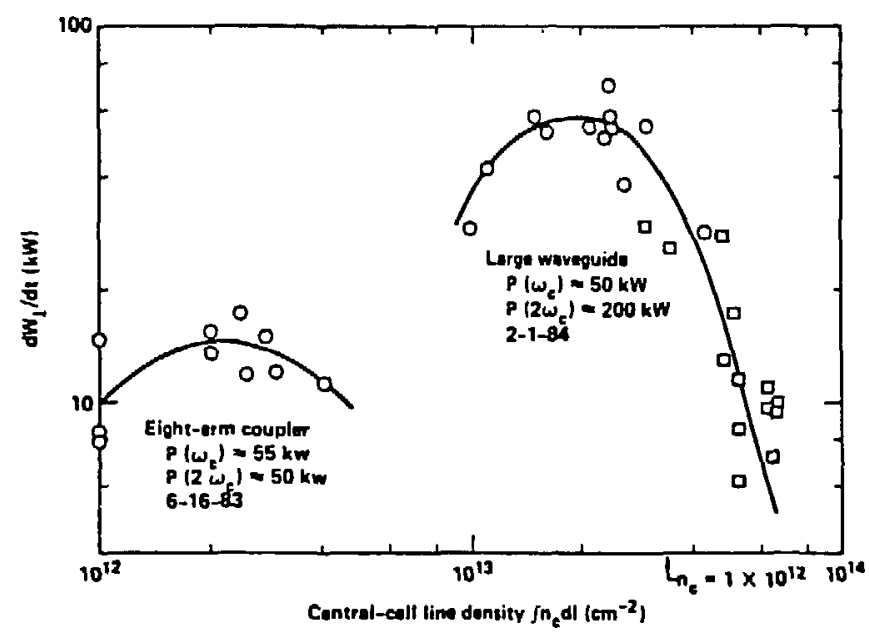

Figure IX.B.1. Variation of mirror-trapped electron heating rate in TMX-Upgrade end cell as a function of center-cell density and microwave power. 
the fundamental and second harmonic heating systems, respectively. Only data acquired during the time that both systems were operating is used because often there was an abrupt decrease in the heating efficiency when the fundamental power went off. During this time window, 20 to $55 \mathrm{msec}$, the data was averaged over one millisecond intervals. A total of over 350 heating efficiency values were used in the analysis. If this efficiency is simply plotted as a function of center cell density, little correlation can be seen; see the upper half of figure IX.C.1.

A correlation can be observed when the data is divided into subsets according to the value of the associated diamagnetic loop signal for each point. The lower half of figure IX.C.1 shows the center cell electron density values plotted versus the west plug diamagnetic loop, and the vertical bands correspond to four different subsets. Plots of heating efficiency versus center cell density corresponding to data in the different bands or subsets are shown in figures IX.C.2 and 3. A correlation is clearly seen, and the dependence of the heating efficiency is different, depending on the amount of energy already stored in the mirror-trapped electrons. When the stored energy is low, 1 to $2 \mathrm{~J} / \mathrm{cm}$, the expected peaking at moderate densities is obtained. This is shown in the upper half of figure IX.C.2. The peak efficiency, $26 \%$, occurs at about $5 \times 10^{11} \mathrm{~cm}^{-3}$, which agrees with the previous analysis. There is not, however, the strong drop as one goes to higher densities; rather the heating efficiency drops by about 30 to $40 \%$ and remains at that ?evel, approximately $17 \%$, up to densities of $1.6 \times 10^{12} \mathrm{~cm}^{-3}$. The clumping of the data into four 

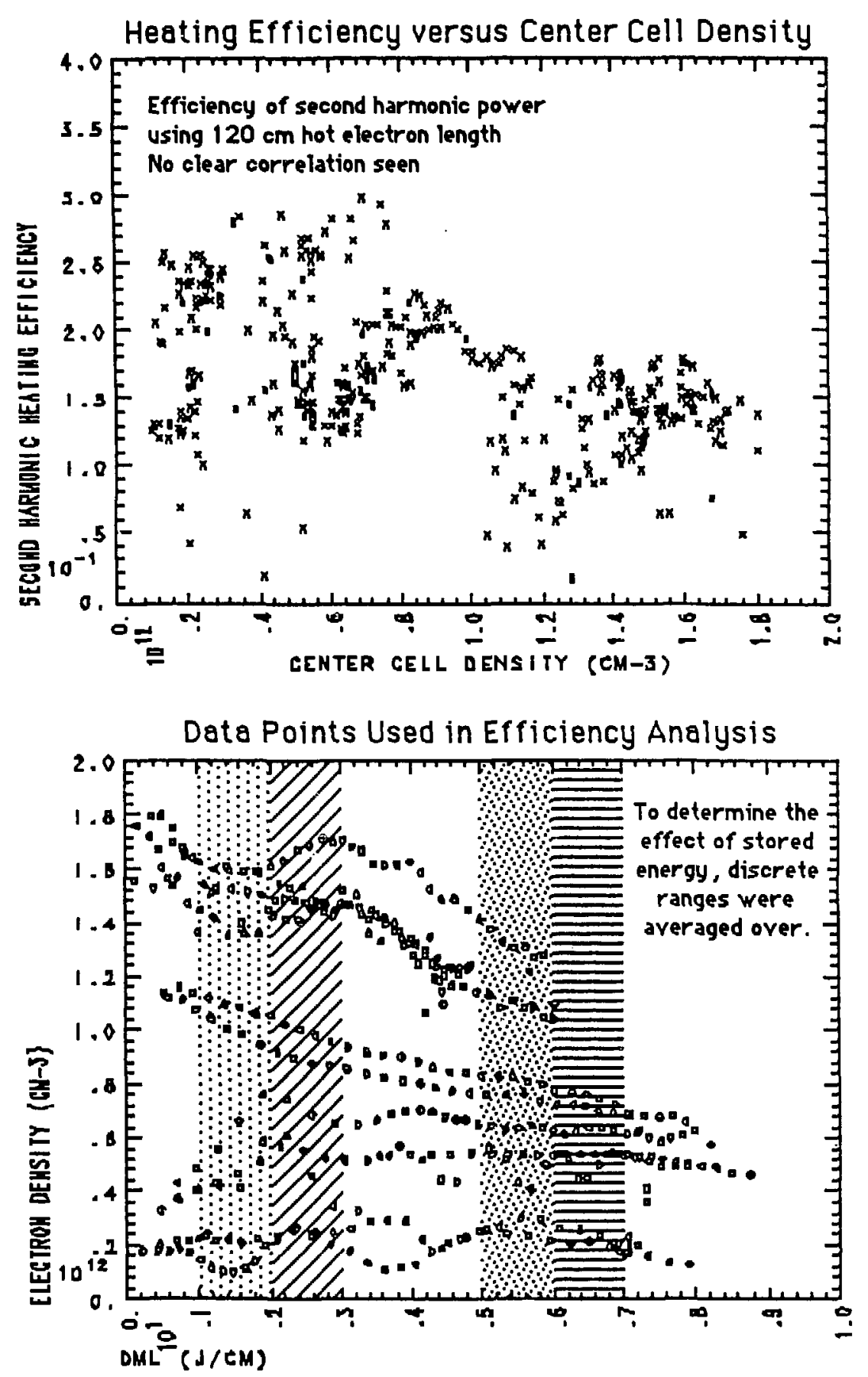

Figure IX.C.1. Heating efficiency of the second harmonic microwaves depends on stored energy as well as cold electron feed (from center cell). To determine the effect of stored energy, discrete ranges were examined individually. 
Data from 1 April 1985. Both fundamental $(100 \mathrm{~kW})$ and second harmonic $(140 \mathrm{~kW})$ systems on. Time window for data was 20 to $55 \mathrm{msec}$.
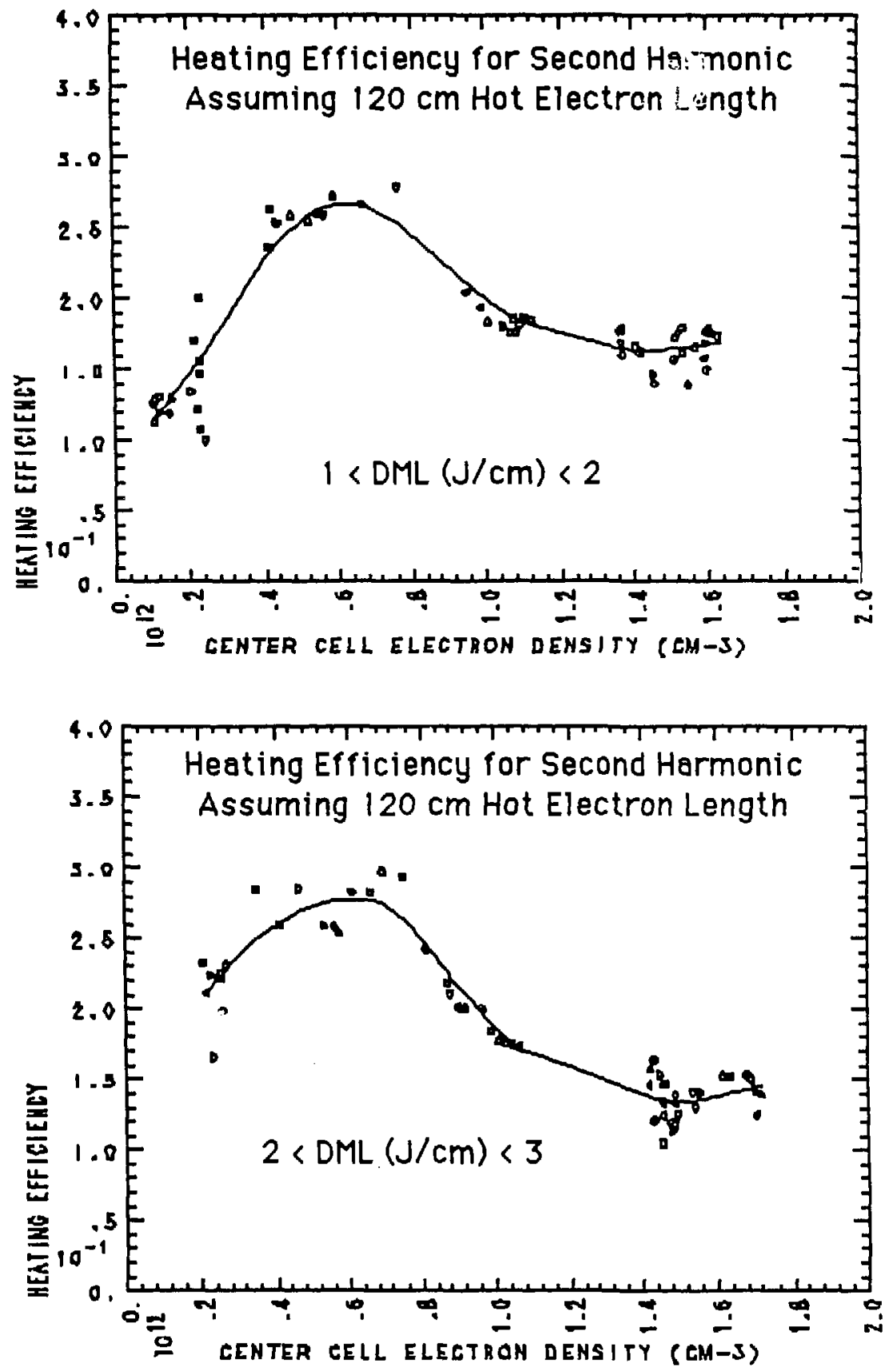

Figure DX.C.2, Heating efficiency as a function of center cell density when the stored energy is low. 
groups is due to the fact that four different gas feed rates were used for the data analyzed.

When the stored energy is high, the efficiency for heating electrons peaks at a lower center cell density, $\sim 2 \times 10^{11}$ as compared to $5 \times 10^{11} \mathrm{~cm}^{-3}$, and can be as high as $25 \%$. (Plots of heating efficiency versus center cell density for the stored energy ranges of 5 to 6 and 6 to $7 \mathrm{~J} / \mathrm{cm}$ are shown in figure IX.C.3.) This peaking at lower center cell denitins is consistent with an increased absorption efficiency for the end cell inirror-trapped electrons, and a reduced absorption efficiency for the electrons in the cold stream flowing out of the center cell. Because both cold and mirsor-trapped electrons are present in the region where the microwaves are being applied, both groups can potentially absorb the incident microwave power-the cold electrons at the resonance location, and the mirror-trapped electrons anywhere that the Doppler-shifted resonance condition is satisfied. However, the cold resonance has shifted from a location near the machine axis out to a radius where the drifts can be poor. Because the pitch angle requirement for confinement at large radii is more stringent, the probability that a cold particle will be trapped is reduced. On the other hand, the absorption efficiency of the already mirror-trapped electrons is increased because there are simply more of them. 
If the confinement time of the trapped electrons is long compared to the length of the heating pulse, the variations in the stored energy rate of rise can be equated with a change in the heating efficiency. If $W$ is the energy stored in the mirror-trapped electrons, the time rate of change of $W$ is

$$
\frac{d W}{d t}=\eta P-\frac{W}{\tau}
$$

where $\eta$ is the microwave heating efficiency, $P$ is the microwave power level, and $\tau$ is the energy confinement time. The fact that the energy confinement time is long is verified by the behavior of the stored energy when the second harmonic heating is turned off early in a shot and the plasma continues to 
Data from 1 April 1985. Both fundamental (100 kW) and second harmonic (140 kW) systems on. Time window for data was 20 to $55 \mathrm{msec}$. .
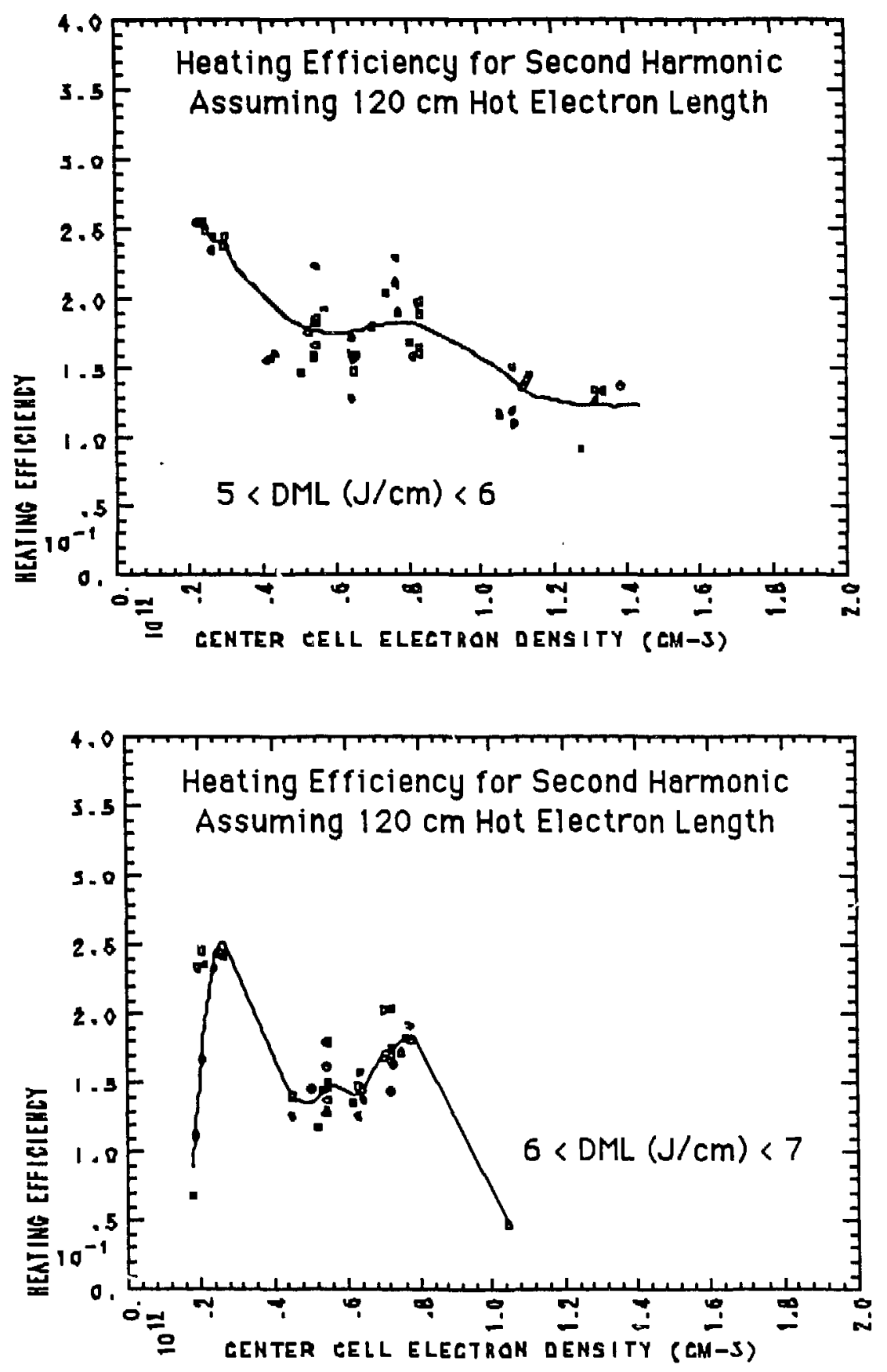

Figure IX.C.3. Heating efficiency as a function of zenter cell density when the stored energy is high. 
be sustained by fundamental heating or neutral beams. An example of such a shot is shown in figure VIII.B.1. Thus it is reasonable to approximate changes in the stored energy rate of rise with variations in heating rate, as long as the other power sources (fundamental microwave heating and neutral beams) remain constant.

\section{Conclusion}

The heating efficiency depends on both the center cell density and the end cell diamagnetism, not just the density alone. Also, because the energy confinement time of the mirror-trapped electrons is long, variations in the diamagnetism rate of rise are directly the result of changes in heating efficiency.

When the stored energy is low, the heating is most efficient for moderate $\left(5 \times 10^{11} \mathrm{~cm}^{-3}\right)$ center cell densities, but when the stored energy is high, that value decreases to $2 \times 10^{11} \mathrm{~cm}^{-3}$. In both cases the peak efficiency, for this data set, is estimated to be about $26 \%$. These results are summarized in figure IX.D.1. This data indicates that the trapping efficiency of the microwaves, i.e. the ability to increase the density of mirror-trapped electrons at the thermal barrier location, degrades as the stored energy increases: once the stored energy has become sufficiently large, further increases can be achieved only by cutting back the cold electron stream and running the trapped electrons up to higher energies. 
Data from 1 April 1985. Both fundamental $(100 \mathrm{~kW})$ and second harmonic $(140 \mathrm{~kW})$ systems on. Time window for data was 20 to $55 \mathrm{msec}$.

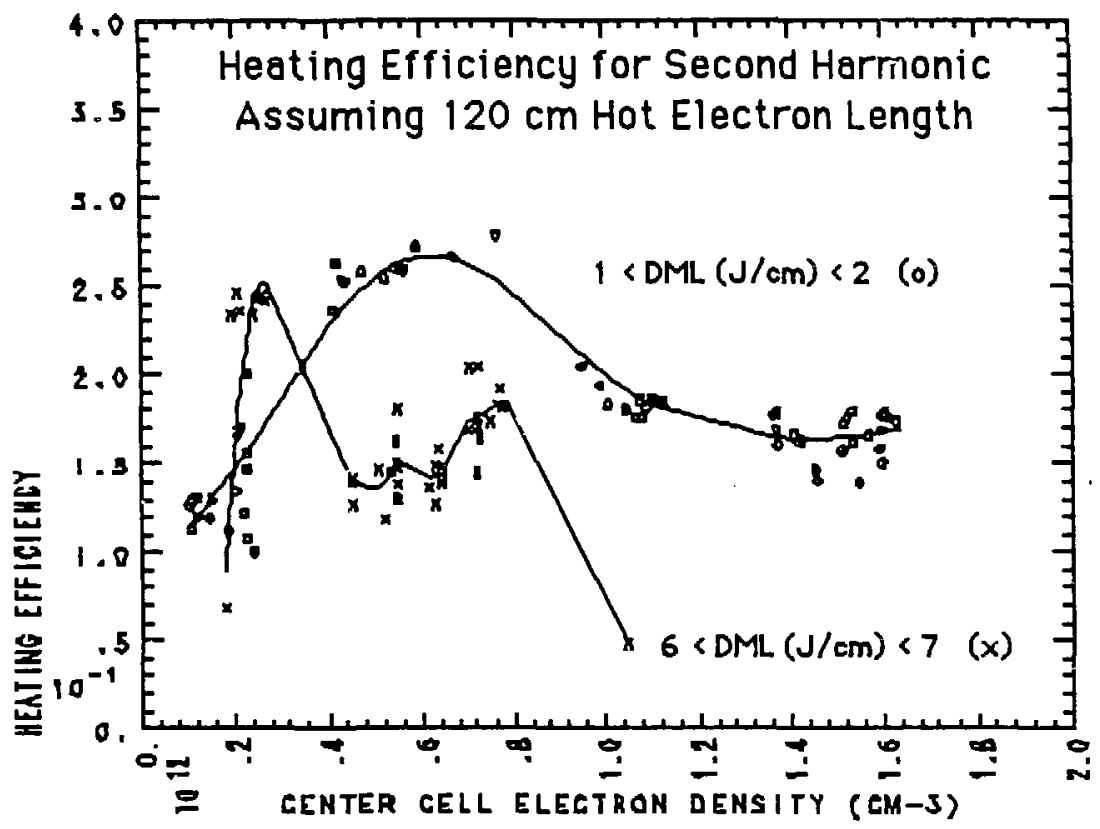

Figure IX.D.1. Heating efficiency as a function of center cell density comparing low and high stored energy cases. 
A degradation of the trapping efficiency when the level of stored energy is high is consistent with microwave absorption away from the machine axis. It has been shown earlier that increases in stored energy are correlated with a broadening of the mirror-trapped electron radial profile. If the heating location has indeed moved out to a larger radius, the heating there might be less efficient for at least two reasons. First, as shown in chapter VII, electrons at large radii which have sufficiently small pitch angles will not be confined, due to poor drifts. Second, there is a much higher probability of scrape-off from probes and limiters at large radii. For these reasons, trapping of electrons from he cold stream is reduced, which is why the heating rate at moderate center cell densities drops as shown in the previous figure. Thus, in order to maintain a high heating efficiency at large center cell densities, it is important to find a way to maintain a cold resonance near the machine axis as the stored energy in the mirror-trapped electrons increases.

During recent TMX-U experiments, long axial confinement times consistent with the formation of a thermal barrier have only been observed for center cell densities below $1.2 \times 10^{12} \mathrm{~cm}^{-3}$. The fact that efficiency of producing mirror-trapped electrons on machine axis drops as the stored energy increases may account for this. This drop in efficiency would effectively limit the mirror-trapped electron density. 


\section{Chapter X}

\section{Conclusion}

\section{A. Introduction}

This work is naturally divided into two parts. The first deals with the design of an instrument that can be used to image bremsstrahlung emission from a mirror-trapped electron plasma, and the second describes the measurements that were made and how they provided an increased understanding of electron cyclotron microwave heating in TMX-U. In this section the conclusions will be summarized and areas of further investigation outlined.

\section{B. High Energy X-ray Imaging}

A method for interpreting the x-ray images was developed and an instrument was successfully designed, built, and used to measure radial and to some extent axial emission profiles from the Tandem Mirror ExperimentUpgrade. In order to obtain an emissivity from the measured intensity, it was necessary to compensate for pinhole collimation, intensifier nonuniformity, and plasma ellipticity. Subsequently the processed intensity was Abel inverted by fitting it to a model for the emissivity profile. The instrument itself consisted of a shielded pinhole and an imaging $\mathrm{x}$-ray detector. The detection efficiency was high due to the fact that $\mathrm{CsI}(\mathrm{Na})$, a scintillator with a high atomic number and subsequently large photoelectric cross-section, was used to convert the $\mathrm{x}$-rays to optical photons. For the $3 \mathrm{~mm}$ crystal, the $1 / e$ efficiency points were 10 and $180 \mathrm{keV}$. The gain of the intensifier chain 
used to amplify the scintillated light was estimated to be $1.9 \times 10^{5}$. This allowed individual $x$ rays with energies as low as $5 \mathrm{keV}$, to be seen at the intensifier output with the naked eye. High speed film (Kodak 2484) and a high sensitivity CCD video camera were used to record the images, the CCD camera providing multiple frames per shot.

A significant upgrade to the detection electronics is underway. These include a more uniform image intensifier with adjustable gain and a silicon intensified target (SIT) vidicon readout of the intensified image. ${ }^{1}$ An entire image will be scanned in less than $5 \mathrm{msec}$, with each image location digitized to eight bit accuracy. The area of the image to be scanned can be dynamically controlled through a computer interface, and at low count rates the system can be operated in a single photon counting mode which will allow energy as well as spatial information to be obtained. The major challenge now will be in developing a way to archive, process, and analyze the enormous amount of experimental data the instrument will produce.

\section{Experimental Analysis}

Analysis of the experimental data obtained from the instrument has provided increased understanding of the electron heating performance. Thick target emissions were linked to heating at large radii where, for particles with small velocity space pitch angles, the drifts are bad. With respect to volume emission from the main plasma, no axial variation was detected over the camera field of view. This is consistent with the fact that the magnetic field gradient is small. Radial variations were observed, however, and these were 
correlated with an increase in stored energy as measured by a diamagnetic loop. When the stored energy was high, the typical gaussian fitted x-ray emissivity radius measured was $16 \mathrm{~cm}$. This would correspond to a mirrortrapped electron radius of $22 \mathrm{~cm}$.

Modifications to the heating systems have been made for the next series of experiments. The second harmonic heating systems have been modified so that they can be aimed to heat at axial locations where the magnetic field gradient is not as flat. This allows a resonance on machine axis to be maintained within the microwave beam profile as the plasma $\beta$ increases, which is necessary if the trapped electron density on axis is to continue growing when the stored energy is large. Finally, $18 \mathrm{GHz}$ microwave heating systems have been added to increase the bulk electron temperature. ${ }^{2}$ These auxiliary heating sources have been added to improve the electron trapping efficiency because warm electrons are more effectively heated by the second harmonic systems. 


\section{List of References}

\section{Chapter I}

1. D. E. Baldwin and B. G. Logan, "Improved Tandem Mirror Fusion Reactor," Phys. Rev. Lett. 43, 1318 (1979).

2. T. C. Simonen, Ed., and the TMX Group, Summary of Rusults from the Tandem Mirror Experiment (TMX), Lawrence Livermore National Laboratory, Livermore, CA, UCRL-53120 (1981).

3. D. E. Baldwin, "Description of the thermal barrier concept," in Summary of TMX-U Results: 1984, T. C. Simonen, Ed., Lawrence Livermore National Laboratory, Livermore, CA, UCID-20274 (1984), Vol. 1, p. 1-12.

4. B. Stallard, "Hot-electron physics," in Summary of TMX-U, Vol. 1, pp. 3-3-11.

5. E. H. Silver, J. F. Clauser, and B. H. Failor, "X-ray diagnostics" in Summary of $T M X-U$, Vol. 2, p. 7-151.

6. W. Nexsen, "Diamagnetic loops," in Summary of TMX-U, Vol. 2, p. 7-122.

7. R. A. James, "Electron-cyclotron emission diagnostic," in Summary of TMX-U, Vol. 2, p. 7-129.

8. E. H. Silver, "Measuring the Mirror-Trapped Electron Fraction in TMXU," Bull. Am. Phys. Soc. 30, 9, paper 812 (1985).

9. T. C. Simonen and B. W. Stallard, "Power scaling of hot-electron beta," in Summary of TMX-U, Vol. 1, p 3-17.

10. R. A. James, "Hot-electron spatial profiles," in Summary of TMX-U, Vol 1, p. 3-15.

\section{Chapter II}

1. W. H. Tucker, Radiation Processes in Astrophysics (MIT Press, Cambridge, MS, 1975), and references therein.

2. E. Haug, "Bremsstrahlung and Pair Production in the Field of Free Electrons," Z. Naturforsch. 30 a (1975), 1099-1113. 
3. E. Haug, "Electron-Electron Bremsstrahlung in a Hot Plasma," Z. Naturforsch. 30 a (1975), 1546-1552.

4. Silver, "Measuring."

5. C. De Michelis and M. Mattioli, "Soft-x-ray spectroscopic diagnostics of laboratory plasmas," Nucl. Fusion 21, 6 (1981).

6. S. von Goeler, W. Stodiek, H. Eubank, N. Fishman, S. Gregenshchikov, and E. Hinnov, "Thermal X-ray Spectra and Impurities in the ST Tokamak," Nucl. Fusion 16, volume number (1975), p. 304.

7. von Goeler, p. 303.

8. E. H. Silver et al., "Soft $x$-ray measurements from the PDX tokamak," Rev. Sci. Instrum. 53, 8 (1982), pp. 1202-1204.

9. E. H. Silver, J. F. Clauser, and B. H. Failor, "X-ray spectroscopy and imaging measurements" in Summary of TMX-U, Vol. 1, p. 3-38.

10. G. F. Knoll, Radiation Detection and Measurement (Wiley, New York, 1979), p. 57.

11. R. D. Evans, The Atomic Nucleus (McGraw-Hill, New York, 1955) pp. 616-7.

12. H. W. Koch and J. W. Motz, "Bremsstrahlung cross-section formulas and related data," Rev. Mod. Phys. 31, 4 (1959), p. 951.

13. Koch, p. 952.

14. H. A. Kramers, Phil. Mag. 46, 836 (1923).

15. National Bureau of Standards (U.S.) Handbook 62 (1957), pp. 20-24.

16. Evans, p. 617.

17. Koch, p. 955.

18. F. F. Chen, Introduction to Plasma Physics (Plenum Press, New York, 1974), p. 26.

\section{Chapter III}

1. Evans, p. 688 .

2. Nikon FE2 Repair Manual (Nippon Kogaku, Tokyo, 1983), p. M2. 
3. D. N. Hill, private communication, Nov 1985.

4. Kinoll, pp. 784-5.

5. K. Kalata, private communication, Dec 1985.

\section{Chapter IV}

1. Knoll, p. 65.

2. Evans, p. 717.

\section{Chapter V}

1. J.T. Routti and J.V. Sandberg, "General Purpose Unfolding Program LOUH178 with Linear and Nonlinear Regularizations", Computer Physics Communications 21 (1980) pp. 119-144.

2. L. Fox and J. H. Wilkinson, NAG Fortran Library Manual, Mark 8 (Numerical Algorithms Group, Oxford, England, 1981).

\section{Chapter VI}

1. S. L. Allen, "Impurity measurement and control," in Summary of TMX$U$, Vol. 1, p 4-134.

2. S. L. Allen, private communication, Oct 1985.

\section{Chapter VII}

1. Chen, p. 24.

2. R. S. Devoto, private communication, Nov 1985.

3. G. Schmidt, Physics of High Temperature Plasmas (Academic Press, New York, 1979), p. 9 ff.

4. J. A. Byers, private cummunication, Nov 1985.

5. T. L. Yu, S. L. Allen, H. W. Moos, and T. J. Nash, "Impurity Transport in TMX-U," Bull. Am. Phys. Soc. 30, 9, paper 3R25 (1985). 
6. G. L. Francis, R. E. Aamodt, D. A. D'Ippolito, J. R. Myra, and P. J. Catto, "Recent Progress in Magnetic Optimization," Bull. Am. Phys. Soc. 30, 9, paper 1R21 (1985).

7. J. W. Meier, "Electron beam welding characteristics of several materials," in Proceedings Third Symposium on Electron Beam Technology, Mar 23-24, 1961, Boston, Mass., R. Bakish, Ed., (Alloyd Electronics, Boston, 1961), pp. 145-156.

8. R. H. Cohen, Phys. Fluids 26, 2774 (1983).

\section{Chapter VIII}

1. P. R. Bevington, Data Reduction and Error Analysis for the Physical Sciences (McGraw-Hill, New York, 1969), pp. 189-190.

2. James, "Hot-electrons," pp. 3-16-20.

3. W. L. Pickles, "Neutral Fueling Pressure Measurements and Modeling Near the Plasma Edge in TMX-U," J. Vac. Sci. Technol. 3, 3 (1985), Part II, pp. 1171-1174.

3. D. R. Slaughter and M. Derzon, "Neutron Measurements," in Summary of TMX-U, Vol. 2, p 7-156.

\section{Chapter IX}

1. B. W. Stallard, V. Matsuda, and W. M. Nevins, Nucl. Fus. 23, 213 (1983).

2. B. W. Stallard, "Source density scaling," in Summary of TMX-U, Vol. 1, pp. 3-50-51.

\section{Chapter $\mathbf{X}$}

1. K. Kalata, "Highly versatile computer-controlled television detector system," SPIE 331, Instrumentation in Astronomy IV (1982).

2. R. D. Horton, R. A. James, B. W. Stallard, "Fundamental ECH at 18 GHz on TMX-U," Bull. Am. Phys. Soc. 30, 9, paper 3R32 (1985). 